UNIVERSIDADE DE SÃO PAULO

INSTITUTO DE PSICOLOGIA

DEPARTAMENTO DE PSICOLOGIA CLÍNICA

ALESSANDRA VILLAS-BÔAS

Efeitos de análises de contingências sobre Comportamentos Clinicamente Relevantes e sobre mudanças extra sessão (versão corrigida) 


\section{ALESSANDRA VILLAS-BÔAS}

\section{Efeitos de análises de contingências sobre Comportamentos Clinicamente Relevantes e sobre mudanças extra sessão}

Tese apresentada ao Instituto de Psicologia da Universidade de São Paulo como parte dos requisitos para a obtenção do Grau de Doutor em Psicologia.

Área de Concentração: Psicologia Clínica Orientadora: Profa. Dra. Sonia B. Meyer Co-orientador: Prof. Dr. Jonathan W. Kanter 
AUTORIZO A REPRODUÇÃO E DIVULGAÇÃO TOTAL OU PARCIAL DESTE TRABALHO, POR QUALQUER MEIO CONVENCIONAL OU ELETRÔNICO, PARA FINS DE ESTUDO E PESQUISA, DESDE QUE CITADA A FONTE.

Catalogação na publicação

Biblioteca Dante Moreira Leite

Instituto de Psicologia da Universidade de São Paulo

Villas-Bôas, Alessandra.

Efeitos de análises de contingências sobre comportamentos clinicamente relevantes e sobre mudanças extra sessão. / Alessandra Villas-Bôas; orientadora Sônia Beatriz Meyer . -- São Paulo, 2015.

$200 f$.

Tese (Doutorado - Programa de Pós-Graduação em Psicologia. Área de Concentração: Psicologia Clínica) - Instituto de Psicologia da Universidade de São Paulo.

1. Terapia comportamental 2. Análise de contingências 3. Generalização 4. Medidas externas 5. Delineamento experimental 6 . Categorização I. Título.

RC489.B4 
Nome: Villas-Bôas, Alessandra

Título: Efeitos de análises de contingências sobre Comportamentos Clinicamente Relevantes e sobre mudanças extra sessão

Tese apresentada ao Instituto de Psicologia da Universidade de São Paulo como parte dos requisitos para a obtenção do Grau de Doutor em Psicologia

Aprovada em:

Banca Examinadora

Prof. Dr.

Instituição: Assinatura:

Prof. Dr.

Instituição: Assinatura:

Prof. Dr.

Instituição: Assinatura:

Prof. Dr.

Instituição: Assinatura:

Prof. Dr.

Instituição: Assinatura: 
Dedico com muito amor esse trabalho ao meu Gelvam, que com seu carinho, apoio, incentivo e compreensão, permitiu meu crescimento nesse período. 


\section{AGRADECIMENTOS}

Algumas pessoas têm feito parte da minha vida nos últimos anos (ou há muito mais tempo) e muito contribuíram pra condução desse trabalho. Gostaria então de mencionar meus sinceros agradecimentos:

À minha querida orientadora, Sonia Meyer. Sonia, é muito bom concluir essa caminhada ao seu lado. Desde o início da nossa relação, há 13 anos (já!) você tem sido um exemplo pra mim de respeito, afeto, compaixão e sensibilidade. Sem você talvez eu fosse arquiteta hoje em dia... Lembra? Nunca me esquecerei da forma como você me acolheu na minha dúvida e insegurança quando te procurei em crise por não saber se era capaz de ser psicóloga clínica ou até mesmo psicóloga. E agora termino essa jornada ao seu lado. Obrigada por não ter desistido de mim e ter me acolhido nas horas em que mais precisei. Começando com supervisões individuais, passando pelas deliciosas supervisões em grupo de 5 a e culminando nesse doutorado. É bom demais ter você em minha vida semanalmente por mais de dez anos. Você me formou como psicóloga clínica, que observa, compreende, desconfia, acolhe, guia, modela, muito além dos primeiros passos. Graças a você estou onde estou...! Obrigada!

Ao meu querido co-orientador, Jonathan Kanter. Jonathan, graças a você tenho alcançado conquistas com as quais jamais sonhei, relacionadas tanto a meu desenvolvimento profissional, como também pessoal. Obrigada por sempre ter acreditado em mim e por sempre ter me contado sobre isso. É maravilhoso tê-lo fazendo parte da minha vida. Sem dúvida você foi um dos melhores presentes que esse doutorado me trouxe! Obrigada por tudo que tem compartilhado comigo e por toda a orientação que você tem me dado nesses últimos anos, não só nesse doutorado.

Ao Glenn Callaghan por ter sempre considerado essa pesquisa super cool! e ter super me incentivado em minhas ideias!

É com muito carinho e gratidão que não posso deixar de mencionar minhas queridas clientes, Dora, Sol e Carla. Os nomes são fictícios, mas vocês se reconhecem neles. Graças a disponibilidade e confiança de vocês, foi possível o desenvolvimento desse trabalho. Jamais vou esquecer as particularidades, alegrias e sofrimentos que dividiram comigo, doando parte da nossa história para a presente pesquisa. Um abraço apertado para cada uma de vocês.

A Claudia Oshiro, Natália Fonseca, Ester Mendes, Paulo André Sutti e Anna Carolina Engelke por terem sido tão responsáveis, cuidadosos e respeitosos nas ajudas oferecidas nesse trabalho. Agradeço muito pelo tempo despendido!

Às queridas amigas Marcia Kameyama, Claudia Oshiro (novamente!) e Milena Geremias. Marcia (ou melhor, Marida!) sua companhia nesse período foi essencial para lidar com todas as transformações pelas quais passei, sem você tudo teria sido muito mais difícil...! Obrigada por ser um exemplo, uma companhia, um ombro, uma risada e tantas outras coisas maravilhosas, sempre na hora certa. Já lhe disse inúmeras vezes e repito aqui: eu não sei o que é maior, meu amor ou minha admiração por você! Clau, boa e querida amiga, colega, professora, co-líder, companheira de conversas, desabafos, crises e paranoias! Sempre foi ótimo me esquivar das categorizações para darmos risadas juntas...! Seus conselhos profissionais e acolhimento pessoal, ajudaram imensamente. Mi, você foi mais um dos lindos presentes que o doutorado me trouxe. Você entrou 
na minha vida tão rapidamente e por aqui ficou... Que delícia! Trocar experiências de vida e profissionais com você sempre foi uma das minhas atividades favoritas.

Aos demais colegas de laboratório e reuniões de quinta: Carol Franceschini, Dani Tsubota, Jan Leonardi, Ligia de Carvalho, Mari Castelli, Natália Fonseca, Paty Rossi, Rodrigo Xavier, Tauane Gehm, Victor Mangabeira, e tantos outros que já passaram por esse grupo! Foi muito bom tê-los como interlocutores e companheiros em todos esses anos!

Aos meus queridos colegas e amigos americanos que me receberam e acolheram tão bem em minhas estadas em Milwaukee e Seattle e tornaram esse período em país estrangeiro, com língua, comidas e costumes diferentes uma experiência incrível! Adam Kuczynski, Ajeng Puspitasari, Cristal Weeks, David Baruch, Gaby Nagy, Joe Murphy, María Santos, Tara Elisabeth, Will Bowe, cada um de vocês tem um espaço especial no meu coração.

Aos amigos Bob Kohlenberg, Fátima Conte, Gareth Holman, Joanne Steinwachs, Mary Loudon, Mavis Tsai, Renato Molina e Yara Ingberman. O apoio e confiança que vocês depositaram em mim, de diversas formas e em diferentes momentos, fizeram muita diferença!

À querida Taninha e Adriana que com seus almoços rápidos, eficientes e atenciosos me salvaram muitas vezes de buracos no estômago e enxaqueca em meio a coleta, categorizações, reuniões... E aos funcionários da secretaria, em especial Claudia e Moisés por terem sempre clareado todos os formulários e burocracias!

Às minhas duas queridas famílias americanas, que sempre me receberam tão bem, cuidando de mim das mais diversas formas possíveis, Katie, David, Zohreh e Saphira; Gwynne, Jonathan e Zoe. Obrigada por terem feito eu me sentir em casa!

Aos meus pais Edgar e Sandra por terem sempre investido e lutado para que eu tivesse uma educação sólida, e também aos meus irmãos Alexey e Cyrus. Obrigada por terem me dado toda a base necessária para todas essas conquistas!

Ao meu grande amor e companheiro de vida já há quase uma década, meu porto seguro, Gelvam Hartmann. Gê, é impossível imaginar alguém melhor para caminhar junto, acompanhando e incentivando meu crescimento, minhas conquistas e minhas intermináveis horas de categorização e escrita; e também cuidando do meu stress, meu cansaço. Sua compreensão, carinho, respeito e apoio permitiram que eu conquistasse muito mais do que esperava. Obrigada por ter estado ao meu lado por todo esse caminho e por tudo o que virá pela frente. Como você sabe, estar em casa é estar nos seus braços. Te amo!

À Universidade de São Paulo e à University of Wisconsin-Milwaukee por terem me recebido e acolhido.

À Fundação de Amparo à Pesquisa do Estado de São Paulo, pela concessão da bolsa de doutorado e pelo apoio financeiro para a realização desta pesquisa. 
Renda-se, como eu me rendi. Mergulhe no que você não conhece como eu mergulhei. Não se preocupe em entender, viver ultrapassa qualquer entendimento.

O que importa afinal, viver ou saber que se está vivendo?

Clarice Lispector 


\section{RESUMO}

Villas-Bôas, A. (2015). Efeitos de análises de contingências sobre comportamentos clinicamente relevantes e sobre mudanças extra sessão. Tese de Doutorado, Departamento de Psicologia Clínica, Instituto de Psicologia, Universidade de São Paulo, São Paulo.

A Psicoterapia Analítica Funcional (FAP) tem o objetivo de trabalhar com as dificuldades do cliente através da relação terapêutica, reconhecendo três principais tipos de comportamento do cliente que podem ocorrer em sessão: CCR1 (comportamentoproblema), CCR2 (comportamento de melhora) e CCR3 (comportamento de análise). Durante a interação terapêutica, o terapeuta deve estar atento aos CCRs do cliente (Regra 1), evocá-los (Regra 2) e responder a eles de forma natural, principalmente ao reforçar os CCR2s (Regra 3), verificar o efeito do seu comportamento sobre os CCRs (Regra 4) e utilizar estratégias de generalização através de análises funcionais sobre o comportamento do cliente, ou através da solicitação de tarefas de casa que ajudem a promover mudanças no dia a dia do cliente (Regra 5). É possível dividir a interação terapêutica realizada na FAP em duas partes: experiencial (que englobaria Regras 1 a 4; CCR1 e CCR2 do cliente); e analítica (que englobaria a Regra 5 bem como CCR3 do cliente). O objetivo do presente trabalho foi o de verificar se as análises existentes na parte analítica são necessárias, ou ao menos auxiliares, na condução da FAP e, em especial, na transferência de aprendizagem ocorrida em sessão para fora dela. Para isso, foi realizado com duas clientes um delineamento experimental de caso único, do tipo A-B-BC-B-BC, no qual A correspondeu a terapia comportamental sem o uso sistemático da FAP; B correspondeu a inserção das Regras 1 a 4 da FAP; e BC a manutenção das Regras 1 a 4 e acréscimo da Regra 5. Foi realizada uma sessão de follow-up, com condução semelhante a Fase A, cerca de seis meses depois de encerrado o procedimento, a fim de se verificar a manutenção dos ganhos obtidos com o procedimento. As sessões foram filmadas e posteriormente categorizadas com o sistema FAPRS, analisando-se o comportamento de terapeuta e clientes dentro da sessão experimental. Além disso, dados foram coletados fora da sessão terapêutica, através de registro externo das clientes, de um observador externo para uma das clientes e da aplicação do instrumento EAS-40. Melhoras intra e extra sessão foram alcançadas com a condução do procedimento e mantidas até a sessão de follow-up. Foi observado que emissões de Regra 5 na Fase BC, influenciaram o comportamento das clientes de analisar seus próprios comportamentos, incluindo a relação terapêutica. No entanto, não foi observado um efeito diferencial da Regra 5 sobre CCR1 e CCR2 das clientes e tampouco sobre os comportamentos problema e de melhora fora da sessão. Discute-se que diante de um processo claro de modelagem como o conduzido na FAP, análises emitidas por terapeuta ou cliente não pareceram ser necessárias. Talvez tais análises auxiliem nas melhoras obtidas, mas ao menos na presente pesquisa, não o fizeram de forma expressiva e inquestionável. Discussões ainda são levantadas a respeito de instrumentos de coleta sobre melhoras externas e as vantagens que que podem ser alcançadas ao se realizar coletas de dados por diversas fontes, incluindo relatos em sessão de episódios de melhora ou problema externo.

Palavras-chave: Terapia Comportamental, Análise de contingências, Generalização, Medidas externas, Delineamento experimental, Categorização. 


\begin{abstract}
Villas-Bôas, A. (2015). Effects of analyses of contingencies on clinically relevant behaviors and out of session changes. Doctoral Dissertation. Clinical Psychology Department. Psychological Institute. University of São Paulo. São Paulo.
\end{abstract}

Functional Analytic Psychotherapy (FAP) is a behavior analytic, therapeutic approach that addresses the client's difficulties through the therapeutic relationship, recognizing three main kinds of client behaviors that can occur in session: CRB1 (problem behaviors), CRB2 (behavioral improvements) and CRB3 (analytic behavior). During the therapeutic interaction, the therapist should watch for the client's CRBs (Rule 1), evoke them (Rule 2 ) and respond to them in a natural way, specially reinforcing CRB2s (Rule 3), observing the effects of his/her own behavior on CRBs (Rule 4) and working on generalization strategies, which include analyses of the client's behavior or asking him/her to do homework, promoting changes in the client's daily life (Rule 5). It is possible to break the FAP therapeutic interaction into two parts: experiential (including Rules 1 to 4 and the client's CRB1s and CRB2s) and analytic (including Rule 5 and the client's CRB3s). The goal of this investigation was to verify if the analytic part is indeed needed or at least helpful in conducting FAP, especially with respect to the transfer of learning from insession to out-of-session (generalization). For this purpose, two clients were submitted to a single-case experimental procedure, A-B-BC-B-BC, in which A corresponded to behavior therapy without using FAP systematically; B corresponded to the introduction of Rules 1 to 4; and BC to the maintenance of Rules 1 to 4 and the addition of Rule 5. A follow-up session, similar to Phase A, was conducted around six months after the procedure was finished, to verify the maintenance achieved with the procedure. The sessions were taped and coded with the FAPRS system, which analyzes the therapist's and client's behaviors in the experimental session. In addition, out-of-session data were collected, using a client diary card, recordings from an external observer for one of the clients, and by administering the EAS-40. Improvements in and out of session were achieved with the experimental procedure and were maintained until the follow-up sessions. Instances of Rule 5 in the BC phases influenced the clients' analyses of their own behavior, including analyses of the therapeutic relationship. However, a differential effect of Rule 5 on the rates of CRB1 and CRB2 or out-of-session improvements or problems was not observed. It was discussed analyses made by therapists or clients do not seem to be necessary in addition to the clear shaping process conducted by FAP. Maybe these analyses can be helpful for the improvements, but with this research they did not appear to be helpful in an explicit and unquestionable way. In addition, the external measurement of outside improvements and the advantages that can be achieved by the collecting data from different sources, including in session reports of problems or improvements, was discussed.

Keywords: Behavior Therapy, Contingency Analysis, Generalization, External Measures, Experimental Design, Coding. 


\section{LISTA DE FIGURAS}

Figura 1: Porcentagem de emissão das categorias referentes às Regras da FAP, dentre todas as respostas emitidas pela terapeuta, por fase experimental e sessão de follow-up com as clientes Dora e Sol.

Figura 2: Porcentagem de emissão das categorias referentes às Regras da FAP, dentre todas as respostas emitidas pela terapeuta e porcentagem de emissão de categorias referentes aos CCRs (soma de CCR1, CCR2 e CCR3), dentro todas as respostas emitidas pelas clientes Dora e Sol, ao longo das sessões experimentais $(\mathrm{F}=$ follow-up $)$. 94

Figura 3: Porcentagem de emissão das categorias referentes a cada uma das Regras da FAP, dentre todas as respostas emitidas pela terapeuta ao longo das sessões experimentais com as clientes Dora e Sol. A Regra 3 encontra-se em uma escala diferente das demais (à esquerda). As escalas dos gráficos são individualizadas para cada participante ( $\mathrm{F}=$ followup).

Figura 4: Porcentagem de emissão das categorias referentes à CCR1 e CCR2, dentre todas as respostas emitidas pelas clientes Dora e Sol, ao longo das sessões experimentais. As escalas dos gráficos são individualizadas para cada participante $(\mathrm{F}=$ follow-up $)$.... 99

Figura 5: Porcentagem de emissão das categorias referentes à CCR1 e CCR2 (barras), dentre todas as respostas emitidas pelas clientes Dora e Sol e porcentagem de emissão das Regras 2 e 3 (TRB1 e TRB2), dentre todas as respostas emitidas pela terapeuta (linhas) ao longo das sessões experimentais. As escalas dos gráficos são individualizadas para cada participante $(\mathrm{F}=$ follow-up $)$.

103

Figura 6: Porcentagem de emissão da categoria referente à Regra 5 (linha), dentre todas as respostas emitidas pela terapeuta e porcentagem de emissão das categorias referentes às análises realizadas pela cliente Sol (soma de CCR3 e O3 - barra), dentre todas as categorias emitidas pelas clientes Dora e Sol, ao longo das sessões experimentais. As escalas dos gráficos são individualizadas para cada participante $(\mathrm{F}=$ follow-up $)$. 106

Figura 7: Frequência média de emissão das subcategorias referentes ao conteúdo das análises realizadas pela terapeuta (T - Regra 5 - barras) e pelos clientes (C - CCR3 e O3 - linhas), divididas em paralelo, C3 (dentro de sessão) e O3 (fora de sessão), emitida em cada fase experimental, para as clientes Dora e Sol. As escalas dos gráficos são individualizadas para cada participante.

Figura 8: Frequência média de emissão das subcategorias referentes ao conteúdo das análises realizadas pela terapeuta ( $\mathrm{T}$ - Regra 5 - barras) e pelos clientes (C - CCR3 e O3 - linhas), divididas em análises sobre comportamento problema (A1) ou de melhora (A2), emitida em cada fase experimental, para as clientes Dora e Sol. As escalas dos gráficos são individualizadas para cada participante

Figura 9: Frequência média de emissão das subcategorias referentes à função das análises realizadas pela terapeuta (Regra 5 - barras), divididas em terapeuta (T) analisa, evoca ou consequencia análises realizadas pela cliente e frequência de emissão das análises realizadas pelas clientes Dora e Sol (C - CCR3 e O3 - linha), em cada fase experimental. 
Figura 10: Porcentagem de emissão das categorias referentes à CCR1 e CCR2, dentre todas as respostas emitidas pelas clientes Dora e Sol, por fase experimental e sessão de follow-up. As escalas dos gráficos são individualizadas para cada participante. 120

Figura 11: Média semanal da frequência de emissão das respostas registradas pela cliente Dora, por fase experimental (a resposta Pensamentos Ruminativos passou a ser registradas apenas na Fase B1).

Figura 12: Média da pontuação dada a cada uma das respostas registradas em uma escala de 5 pontos (Raramente - 1; Poucas vezes - 2; Algumas vezes - 3; Muitas vezes - 4; Com frequência -5 ), pelo observador externo, por fase experimental.

Figura 13: Escores obtidos para cada uma das dimensões medidas pela Escala de Avaliação dos Sintomas (Laloni, 2001) e o índice geral global da cliente Dora. A escala foi aplicada 11 vezes e a figura indica o momento do delineamento experimental no qual a aplicação foi realizada. A linha tracejada em cada quadro indica a média da população para o sexo feminino em cada uma das dimensões e a área em cinza, o intervalo de desvio padrão. F corresponde a follow-up. 126

Figura 14: Porcentagem de emissão das categorias referentes a $\mathrm{O} 1$ e $\mathrm{O} 2$, dentre todas as respostas emitidas pela cliente Dora, por fase experimental e sessão de follow-up..... 127

Figura 15: Frequência média de emissão de Episódios de $\mathrm{O} 1$ e Episódios de O2 detectados nas falas da cliente Dora, por fase experimental e sessão de follow-up..... 128

Figura 16: Média da frequência de emissão das respostas registradas semanalmente pela cliente Sol, por fase experimental. 131

Figura 17: Escores obtidos para cada uma das dimensões medidas pela Escala de Avaliação dos Sintomas (Laloni, 2001) e o índice geral global da cliente Sol. A escala foi aplicada 11 vezes e a figura indica o momento do delineamento experimental no qual a aplicação foi realizada. A linha tracejada em cada quadro indica a média da população para o sexo feminino em cada uma das dimensões e a área em cinza, o intervalo de desvio padrão. F corresponde a follow-up.

Figura 18: Porcentagem de emissão das categorias referentes a $\mathrm{O} 1$ e $\mathrm{O} 2$, dentre todas as respostas emitidas pela cliente Sol, por fase experimental.

Figura 19: Frequência média de emissão de Episódios de $\mathrm{O} 1$ e Episódios de $\mathrm{O} 2$ detectados nas falas da cliente Sol, por fase experimental. Repetições de uma mesma resposta emitidas durante a semana não foram contabilizadas. Relatos de problema ou melhora emitidos durante a semana, mas que são parte de um CCR também foram contados.

Figura 20: Porcentagem de emissão de CCR1 e CCR2 (barras - escala à esquerda), dentre todas as respostas emitidas pelas clientes e frequência média de emissão de Episódios de $\mathrm{O} 1$ e Episódios de $\mathrm{O} 2$ (linhas - escala à direita) detectados nas falas das clientes, por fase experimental e sessão de follow-up. As escalas dos gráficos são individualizadas para cada participante. 


\section{LISTA DE TABELAS}

Tabela 1: Códigos utilizados no FAPRS para categorização do comportamento do terapeuta (Callaghan \& Follette, 2008), adaptados.

Tabela 2: Códigos utilizados no FAPRS para categorização do comportamento do cliente (Callaghan \& Follette, 2008), adaptados.

Tabela 3: Hierarquia das categorias do FAPRS (Callaghan \& Follette, 2008), adaptada para a presente pesquisa.

Tabela 4: Subcategorias criadas para Regra 5

Tabela 5: Subcategorias criadas para CCR3 e O3

Tabela 6: Hierarquia das subcategorias criadas para a presente pesquisa.

Tabela 7: Delineamento experimental (Etapa II).

Tabela 8: Índices Kappa (Siegel \& Castellan, 1988) e porcentagens de concordância entre categorizações das aferidoras e pesquisadora.

Tabela 9: Correlações de Spearman entre porcentagem de emissão da Regra 5, dentre todas as respostas emitidas pela terapeuta, e a porcentagem de emissão das categorias referentes a CCR3 e $\mathrm{O} 3$ dentre todas as respostas emitidas por cada uma das clientes. A correlação foi calculada sem os dados da sessão de follow-up.

Tabela 10: Quantidade (n) e probabilidade (\%) de emissões de CCR3, O3 e a soma dos dois por Dora e Sol em resposta a uma Regra 5 da terapeuta, por fase experimental, em Lag 1, Lag 3 e Lag 5 ( $\mathrm{N}=$ quantidade de emissões de Regra 5).

Tabela 11: Quantidade (n) e probabilidade (\%) de emissões de Regra 5 pela terapeuta em resposta a análises de Dora (CCR3+O3) e CCR3 de Sol, por fase experimental, em Lag 1, Lag 3 e Lag 5 ( $\mathrm{N}=$ quantidade de emissões de análise de Dora - CCR3 e O3 e quantidade de emissões de CCR3 de Sol). 


\section{SUMÁRIO}

INTRODUÇÃO. . .16

ARTIGO: The use of Analytic Interventions in Functional Analytic Psychotherapy 17

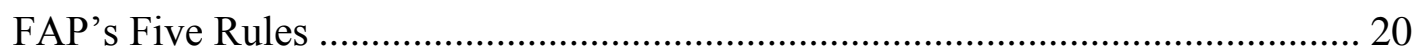

Two Aspects of the FAP Interaction: Experiential and Analytic .......................... 22

The Potential Importance of the Analytic Component of FAP .............................. 24

Definitions of and Relations between Rule 5 and CRB3s ................................... 26

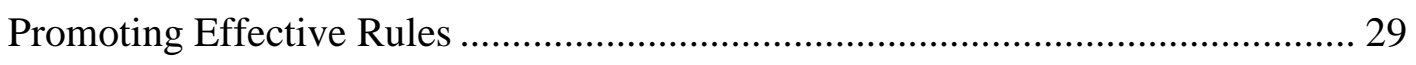

Suggestions for Clinical Implementation of Rule 5 and Shaping of CRB3 ........... 36

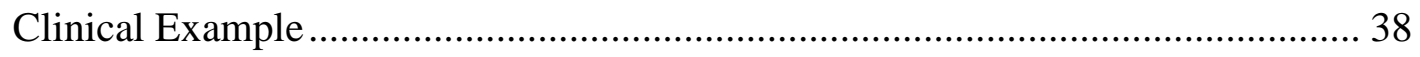

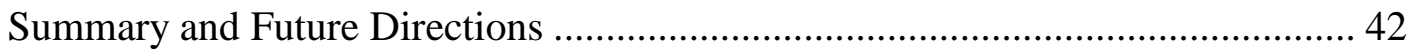

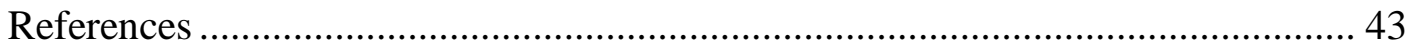

Investigações a respeito dos mecanismos de ação da FAP....................................... 48

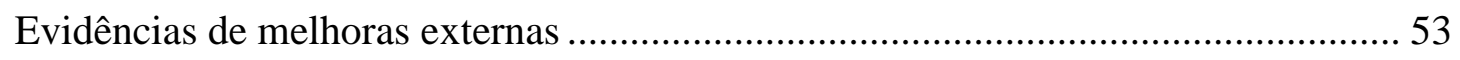

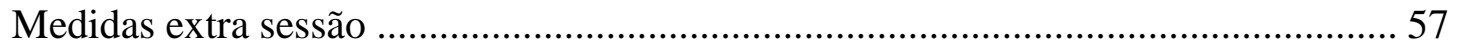

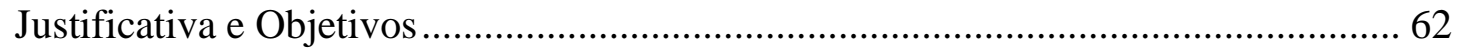

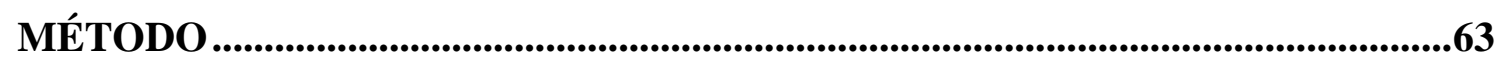

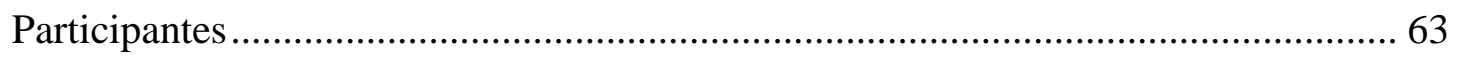

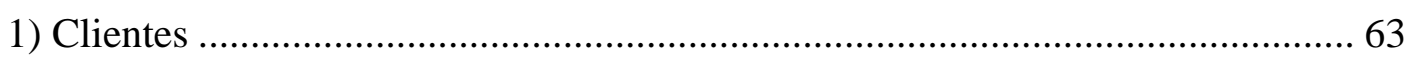

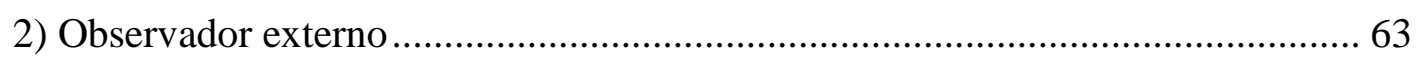

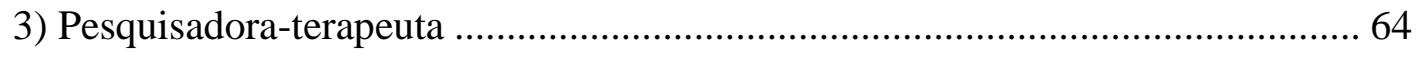

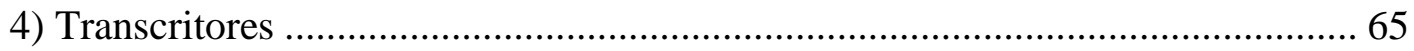

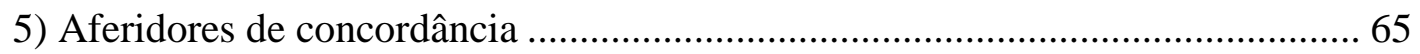

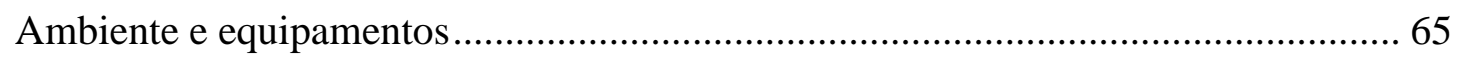

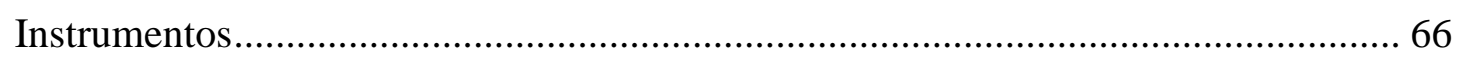

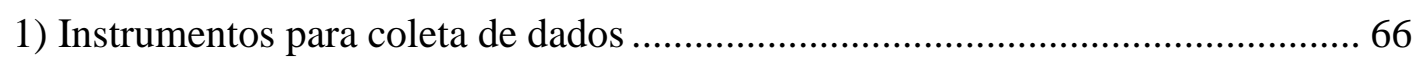

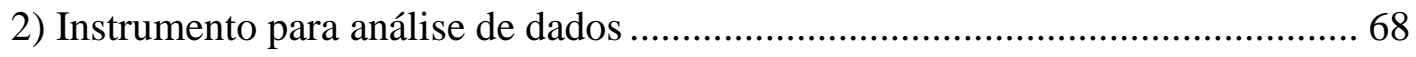

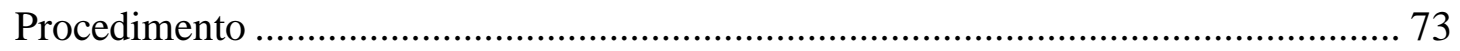

1) Etapa I - Estabelecimento de vínculo ............................................................. 73

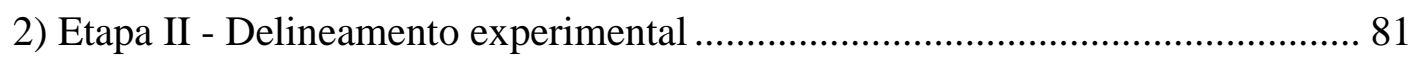

3) Etapa III - Encerramento da coleta e continuidade da terapia e sessão de

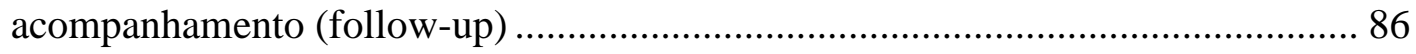

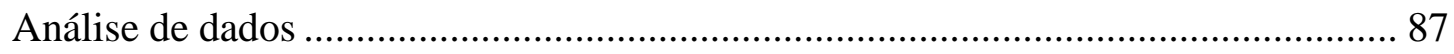




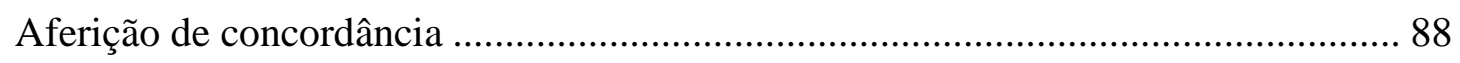

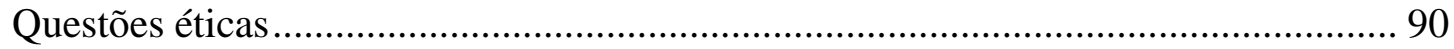

RESULTADOS E DISCUSSÃO ..............................................................................92

A) Evidências de condução do delineamento experimental e os resultados gerais apresentados pelas clientes dentro da sessão terapêutica........................................... 92

B) Os efeitos da Regra 5 sobre comportamentos dentro de sessão........................... 105

B.1) Regra 5 e as análises das clientes ............................................................... 106

B.2) Relação entre análises e os CCR1 e CCR2 …............................................ 119

C) Dados e análises sobre comportamento extra sessão ......................................... 122

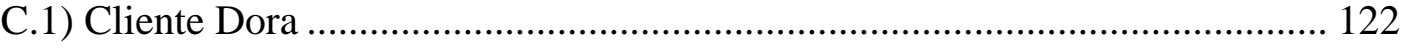

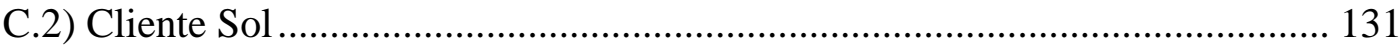

C.3) Resultados externos e decorrentes reflexões .............................................. 138

D) Relações entre respostas intra e extra sessão ....................................................... 144

E) Reflexões adicionais sobre o papel das análises na FAP .................................. 147

Outras possibilidades de contribuição das análises............................................ 152

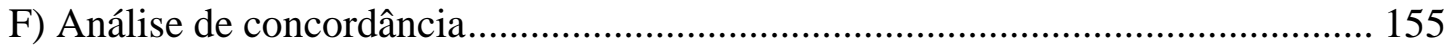

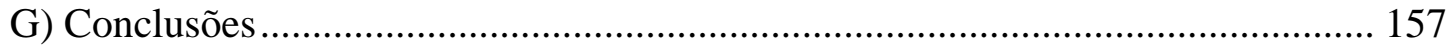

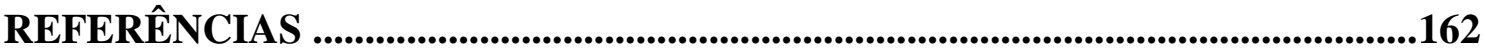

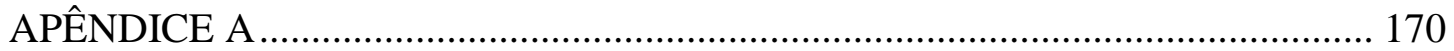

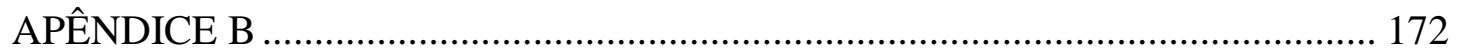

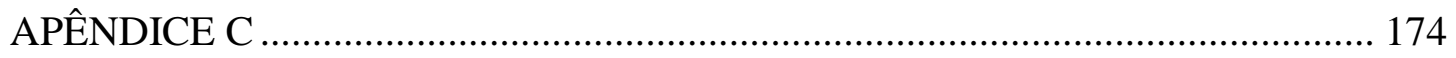

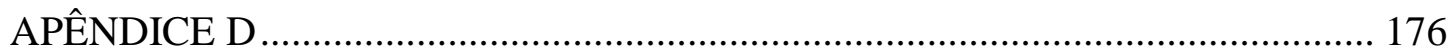

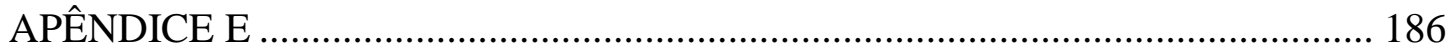

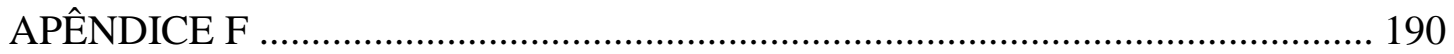

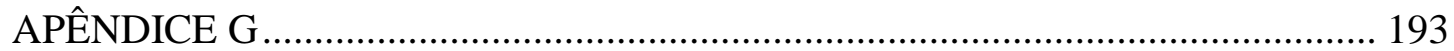

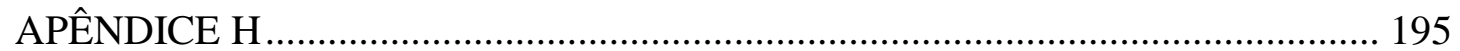

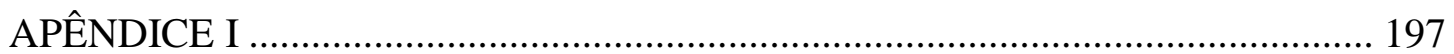


Nos últimos anos, têm crescido o interesse dos psicólogos sobre a Psicoterapia Analítica Funcional (Functional Analytic Psychotherapy - FAP - Kohlenberg \& Tsai, 1991; Tsai et al., 2009; Tsai, Kohlenberg, Kanter, Holman, \& Loudon, 2012). Pesquisadores têm investigado teórica e empiricamente a eficácia dessa psicoterapia (Callaghan, Summers, \& Weidman, 2003; Geremias, 2014; Kanter et al., 2006; Landes, Kanter, Weeks, \& Busch, 2013; Maitland \& Gaynor, 2012; Oshiro, Kanter, \& Meyer, 2012) e também os mecanismos de ação pelos quais a FAP produz seus efeitos (Abreu, Hübner, \& Lucchese, 2012; Busch, et al., 2009; Busch, Callaghan, Kanter, Baruch, \& Weeks, 2010; Lizarazo, Muñoz-Martínez, Santos, \& Kanter, no prelo; Mangabeira, 2014; Meurer, 2011; Popovitz, 2013; Weeks, Kanter, Bonow, Landes, \& Busch, 2012). Recentemente, alguns autores têm discutido a interação entre cliente e terapeuta durante a sessão clínica, nomeando-a como Interação Lógica da FAP (Tsai et al., 2012; Weeks et al., 2012). Refletindo sobre essa interação, é possível dividi-la em duas partes: experiencial (relacionada com os CCRs evocados e modelados) e analítica (relacionada com as análises sobre o comportamento do cliente, feitas tanto por terapeuta como por cliente). A maioria dos trabalhos sobre FAP investiga essa parte experiencial, enquanto a parte analítica não tem sido profunda e extensamente discutida (para uma exceção ver Abreu et al., 2012) ou investigada.

Pretende-se, portanto, na presente Introdução discutir algumas questões relacionadas com análises utilizadas na sessão clínica e como elas podem ajudar na parte experiencial da FAP ${ }^{1}$. Em seguida será feito um levantamento das pesquisas realizadas na área sobre os mecanismos de ação envolvidos na FAP, que servem como base para a presente pesquisa. Por fim, reflexões serão realizadas sobre as formas de coletas de dados fora da terapia, a fim de se identificar formas de medida úteis a serem utilizadas nas pesquisas em FAP.

\footnotetext{
${ }^{1}$ Durante o desenvolvimento da pesquisa um artigo foi escrito pela autora da presente tese em colaboração com os orientadores (Profa. Sonia Meyer e Prof. Jonathan Kanter) e um especialista da área (Prof. Glenn Callaghan) e submetido à publicação. Tal artigo trata de reflexões sobre a parte analítica da FAP e será aqui apresentado na íntegra, seguido do restante dos temas mencionados na Introdução.
} 


\title{
ARTIGO:
}

\section{The use of Analytic Interventions in Functional Analytic Psychotherapy ${ }^{2}$}

\author{
Alessandra Villas-Bôas, M.S. \& Sonia B. Meyer, Ph.D. \\ Universidade de São Paulo \\ Jonathan W. Kanter, Ph.D. \\ University of Washington \\ Glenn M. Callaghan, Ph.D. \\ San Jose State University
}

\begin{abstract}
Functional Analytic Psychotherapy (FAP) is a contextual behavioral approach that uses general principles of behaviorism to improve therapy outcomes. Principles of reinforcement are used to shape the client's behavior in session, strategically reinforcing improvements in the therapeutic relationship. These improvements should extend to the client's personal relationships outside the therapy, because of the functional similarities between in-session and out-of-session contexts. However, the literature suggests that functional analyses about what happens in session can be helpful to increase the chances of extension and transfer of improvements out of session. With that in mind, the FAP process can be separated into 2 parts: experiential and analytical. During the experiential part of the interaction, therapists should evoke and naturally reinforce the client's improvements in a process that can lead to an improvement in intimacy in the therapeutic relationship. During the analytical part, the therapist and client should analyze the interaction that occurred between them in session, creating tracking and augmenting rules, which will help with the client's behavior change out of session. The goal of this article is to discuss how the functional analysis of the therapeutic relationship is important for the extension of in-session improvements to out of session contexts. Discussion of behavioral terms and concepts such as self-knowledge and self-control, rule-governed behavior and behavior shaped by contingencies, sensitivity to changes in contingencies, transformations of stimulus function, generalization, and others, will clarify the theoretical basis for why each of the 2 parts of FAP can potentiate the power of the other.
\end{abstract} Keywords: behavior shaped by contingencies, FAP, rule governed behavior, self-knowledge, stimulus function.

\footnotetext{
${ }^{2}$ Artigo publicado em Behavior Analysis: Research and Practice. Referência completa: Villas-Bôas, A., Meyer, S., Kanter, J., Callaghan, G. (2015). The use of Analytic Interventions in Functional Analytic Psychotherapy. Behavior Analysis: Research and Practice, 15(1), 1-19.
} 


\section{The use of Analytic Interventions in Functional Analytic Psychotherapy}

Functional Analytic Psychotherapy (FAP), first described by Kohlenberg and Tsai in 1991 , is a contextual behavioral approach to adult outpatient psychotherapy that strategically and uniquely uses general principles of behaviorism and reinforcement in the client-therapist relationship in order to maximize therapy outcomes. The primary emphasis on the principle of reinforcement, applied to the client's behavior in the therapy context, suggests that the FAP therapist contingently shapes the client's behavior in session. Because of the functional similarities in client behaviors in and out of session, the response classes that are shaped in session ideally will extend to the client's personal relationships outside the therapy.

The behaviors that are changed via the therapeutic relationship in FAP are called Clinically Relevant Behaviors (CRBs) and are classified as follows: CRB1s are the client's problem behaviors that occur in session; CRB2s are the client's improvements that occur in session; and CRB3s are the client's analyses of his or her own behavior. At the beginning of therapy, it is common to observe a high frequency of CRB1s and a lower frequency of CRB2s and CRB3s (Callaghan \& Follette, 2008), and the goal of the therapy is to decrease CRB1s and to increase CRB2s through the therapist's in-session contingent responding. CRB3s also should increase over the course of successful FAP therapy and are described as important to FAP process in that they help the client specify the relevant contingencies effecting their improved and problematic behaviors. They may also help specify rules to follow in daily life that increase the likelihood of reinforcement of improved daily life behavior. CRB3s are one of the central themes of this paper and will be discussed in detail later.

Several authoritative descriptions of FAP have been published, including the original FAP text (Kohlenberg \& Tsai, 1991) and two updated FAP texts (Tsai et al., 2009; Tsai, Kohlenberg, Kanter, Holman, Loudon, 2012) ${ }^{3}$. Recently, a surge of interest in FAP has occurred. Most of the published works in FAP are theoretical (e.g., Abreu, Hübner, \& Lucchese, 2012; Callaghan, Naugle, \& Follette, 1996; Cautilli, Riley-Tillman, Axelrod, \& Hineline, 2005;Fernández-Parra, \& Ferro, 2006; Follette, Naugle, \& Callaghan, 1996; Holmes, Dykstra, \& Williams, 2003; Hopko \& Hopko, 1999; Kanter, Callaghan, Landes, Busch, \& Brown, 2004; Kohlenberg, Tsai, Parker, Bolling, Kanter, 1999; Pankey, 2012; Weeks, Kanter, Bonow, Landes, \& Busch, 2012; Wetterneck, \& Hart, 2012) but an increase in empirical work on FAP over the last several years was described by Ferro (2008) and Mangabeira, Kanter, and

\footnotetext{
${ }^{3}$ The reader can assume that all general descriptions of FAP are supported by these texts. To avoid redundant references to these texts in the current manuscript, general descriptions of FAP will not include citations.
} 
Del Prette (2012). However, as Mangabeira et al. (2012) observed, the published empirical studies of FAP still lack rigorous designs and most are narrative case studies. Exceptions to this pattern include several detailed process analyses of FAP sessions with successful FAP cases suggesting that the use of specific FAP techniques coincides with improvements in insession behaviors (e.g., Busch, Callaghan, Kanter, Baruch, \& Weeks, 2010; Callaghan, Summers, \& Weidman, 2003; Xavier, Kanter, Meyer, 2012). Across two studies, Kanter and colleagues have shown that adding FAP techniques after a baseline period resulted in improvements in out-of-session behaviors for 4 of 6 clients (Kanter et al., 2006; Landes, Kanter, Weeks, \& Busch, 2013), and a detailed process analysis of one of these successful cases found evidence for specific contingent responding to CRBs and in-session improvements only in the FAP phase as well (Busch et al., 2009). Two single-subject reversal procedures (Maitland \& Gaynor, 2012; Oshiro, Kanter, \& Meyer, 2012) have shown that improvements occur and then attenuate with the addition and then removal of specific FAP techniques, demonstrating FAP's potential for creating behavior change.

Amidst this focused interest in FAP, details of what typically occurs in FAP interactions have been published to clarify what FAP looks like in practice, as well as to facilitate future clinical, research, and training efforts (Weeks et al., 2012). Any description of FAP does not mandate one specific manualized interaction sequence, as the therapist in FAP is charged with reacting functionally and idiographically to the client in the moment. There are some publications that already described important classes of therapist (Callaghan, 2006a) and client (Callaghan, 2006b) behaviors that may be a focus in FAP interventions, but these descriptions are still general classes of behaviors that must be specified to each individual person.

A successful FAP interaction often follows a similar form. This interaction can be divided into two parts: experiential (related to CRBs being directly evoked and consequated in session) and analytic (related to the verbal analysis of the experiential process before or after it has occurred). Most published descriptions of FAP emphasize the experiential part of the interaction, including studies where the therapeutic relationship is coded using FAP Rating Scale categories (FAPRS, Callaghan \& Follette, 2008; Callaghan, Follette, Ruckstuhl, \& Linnerooth, 2008), evidencing the frequency and details of the interactions that occurred in session. In contrast, the analytic part has not been discussed or researched as frequently (Maitland \& Gaynor, 2012). One exception to this is found in the theoretical paper by Abreu et al. (2012), discussing the role of CRB3s in FAP in more detail.

The goals of this paper are to clarify the key issues related to the analytic aspects of FAP and to describe how these analyses can support and even maximize the experiential aspect of 
FAP work. Speculation about the role of the analytic components of FAP can serve to promote research in the area with the aim of empirically verifying the importance of these factors in FAP and specifying how and when these aspects should occur in this treatment. For this purpose, a brief overview of FAP will be followed by a discussion of CRB3s and the use of rules in FAP to maximize therapy outcomes. Finally, a clinical example is presented to suggest how these themes may translate into clinical practice, and future directions for this work are discussed.

\section{FAP's Five Rules}

FAP therapists are taught five basic rules to guide their behavior. These rules will be briefly presented and explained below.

\section{Rule 1: Therapist watches for CRBs (therapeutic awareness)}

In FAP the therapeutic relationship is understood as an important and natural relationship in the client's life. Because of this, it is expected that the client's behavior with the therapist will be functionally similar to the client's behavior with others in his or her daily life, which means that the client's CRBs should occur naturally during the therapeutic relationship. The first therapeutic task, therefore, is to be aware of and watchful for the occurrence of CRBs. In other words, the therapist should pay attention to the client's behavior in session, looking for CRBs by hypothesizing about parallels between in-session observed behavior and out-ofsession behavior reported by the client. This therapeutic awareness and exquisite attention to the client's behavior in the present moment is important in FAP and indicated as Rule 1.

\section{Rule 2: Therapist evokes CRBs (therapeutic courage)}

Rule 2 suggests that the therapist should not only wait for and observe the appearance of CRBs, but the therapist should evoke CRBs directly, to create more opportunities to work with them in session. Evoking CRBs requires therapeutic courage in that it may require bringing up sensitive, uncomfortable, and very personal topics that are difficult for both the client and the therapist to experience in the moment.

Rule 3: Therapist contingently responds to CRBs, focusing on naturally reinforcing CRB2s (therapeutic love)

Rule 3 has been considered the pivotal rule for the mechanism of action of FAP. It consists of responding appropriately to the client's CRBs, which means providing natural consequences contingent on the client's behavior that function to differentially decrease CRB1s and increase CRB2s. Because reinforcement happens immediately after the client's behavior and in the context of an important interpersonal relationship, it should be powerful and result 
in durable changes in the behavior in session. When implementing Rule 3, therapists are encouraged to respond as "naturally" as possible such that similar reinforcement is more likely to occur in the client's outside relationships. On the other hand when the client emits CRB1s, the therapist should respond appropriately in an attempt provide honest, compassionate feedback about the impact of that behavior, and try to evoke and differentially reinforce a CRB2. The use of the term "therapeutic love" as a description of this process of reinforcing CRB2s aims to inform FAP practitioners that therapeutic responses should be deeply caring, compassionate, and tailored to the unique needs of the client.

Rule 4: Therapist observes the potentially reinforcing effects of therapist behavior in relation to CRBs (therapeutic awareness)

After responding to a client's CRBs, FAP theory suggests that it is important that the therapist ascertains that his or her responses did indeed have the intended effect on the client's behavior. In this way, the therapist needs to pay attention to the client's behavior after the interaction has occurred and verify if CRB2s are increasing in frequency or not. This is at the roots of a functional approach to assessment and intervention. If the therapist attempts to reinforce a CRB2, but that class of improved behavior does not occur more frequently in the future, then the behavior was not, in fact, reinforced. The therapist can also ask the client about how he or she felt about the therapist's response and how it could be more helpful in an attempt to investigate what might be more functionally reinforcing for that specific client. Rule 4 hopefully results in the therapist's behavior being shaped by the client's progress, or lack of progress, and helps the therapist to better tailor responses to the unique needs of the client and maximize the likelihood that the FAP process will shape CRB2s in session. In this way, FAP is an iterative process of assessment, responding, determining impact, and then responding again - making necessary adjustments to achieve therapeutic goals.

Rule 5: Therapist provides functionally informed analyses and implements generalization ${ }^{4}$ strategies (therapeutic analysis)

This rule addresses the concern that the in-session improvements must extend from the therapy session to the client's daily life. Traditionally, the basic Rule 5 strategy was to provide descriptions of the contingencies affecting the client's behavior, making these contingencies more salient to the client, and thereby increasing the likelihood that these contingencies will

\footnotetext{
${ }^{4}$ Although in previous FAP writings, the term "generalization" has been used in a loose way to refer to any processes that facilitate the extension of behavior changes that occur in session to out-of-session contexts, Johnston (1979) points out that such imprecise usage clouds our understanding of the processes involved. To be consistent with the FAP literature on Rule 5, we retain this imprecise usage here and clarify the specific processes involved below.
} 
affect his or her behavior. Rule 5 may involve descriptions that relate in-session contingencies experienced by the client and therapist to out-of-session contingencies to help the client see similarities between his behavior and its consequences in session and out of session (in-to-out parallels, Weeks et al., 2012). Rule 5 may also involve designing and asking the client to complete homework assignments to prompt the client to engage in the behavior that was reinforced in session during the between-session intervals.

\section{Two Aspects of the FAP Interaction: Experiential and Analytic}

As discussed by Weeks et al. (2012), a typical FAP interaction consists of FAP's five rules in sequence, which we break into experiential and analytic components. The first part of the interaction can be considered experiential and emotional, as the therapist is fully in the moment with the client, observing and reacting naturally to the client's behavior. For example, the therapist may observe that the client is avoiding difficult emotional material, with the knowledge that avoidance of emotionally laden material is a CRB1 for the client (Rule 1). The therapist may choose to point out and block the avoidance, and encourage the client to discuss the difficult material, thereby attempting to evoke CRB2s (Rule 2). The client may persist in avoidance, and the therapist in turn may gently persist in blocking and evoking. Such loops are common in FAP interactions. The goal, however, is for the client to engage in CRB2s of approaching the aversive content in an emotionally open manner and for the therapist to be naturally reinforcing when this occurs (Rule 3). This natural reinforcement may take the form of the therapist's compassionate, empathetic, or caring reactions, or it may take other forms depending on the unique relationship between the client and therapist. The reinforcement, hopefully, will immediately generate more CRB2s, and this CRB2-Rule 3 loop may occur in the best FAP interactions. Weeks et al. (2012) discuss that this interaction can frequently lead to vulnerability, emotional expression, and improvement in intimacy in the relationship experienced by both the therapist and the client. When the experiential interaction reaches a natural conclusion, the therapist may check on the nature of his or her attempts to be reinforcing in the interaction, observing an increase in CRB2s or asking to the client how the interaction felt to him or her (Rule 4).

After that, the analytic and more rational component of FAP may occur. The therapist may discuss the interaction in detail with the client, attempting to make salient the contingencies that occurred and encourage the client to engage in the behavior out of session (Rule 5). 
Some FAP practitioners may note that Rule 5 may also occur during the experiential, emotional period of the interaction. In fact, Rule 3 and Rule 5 interventions may easily cooccur, as with the therapist immediately responding to a CRB2 of vulnerable self-expression with the comment, "When you are feeling avoidant, but you talk about how are feeling anyway, like you just did, I want you to know that I feel much more connected to you and really appreciate how hard you are working on this here." This is consistent with Rule 5 because it is describing the contingency but it is also consistent with Rule 3 because this comment also hopefully provides reinforcement, or at the least augments other non-verbal reinforcing stimuli that are present in the moment.

As suggested by Weeks et al. (2012), although there are many other possibilities for how a FAP interaction may evolve, there is a logical split in the interaction between Rules 1, 2, and 3, which involve observing, evoking, and reinforcing CRBs, and Rules 4 and 5, which involve talking about what just occurred. In fact, it is suggested in FAP training that rushing in with Rule 4 and Rule 5 interventions (i.e., observing the effect of the intervention and providing an analysis) after Rule 3 (reinforcing client improvements) may function to prematurely, and even problematically, end the naturally reinforcing exchange between the client and therapist and could be an indicator of avoidance on the part of the therapist.

With respect to Rule 5 in particular, it is possible to draw a parallel of the experiential and analytical components of the FAP interaction to Skinner's $(1966 ; 1969 ; 1974)$ distinction between contingency-shaped behavior and rule-governed behavior. Skinner suggests that contingency-shaped behavior makes behavioral control more subtle and precise, and rulegoverned behavior takes advantage of the description of important similarities between different contexts in which behavior might occur. This can be illustrated by his description,

In the forge of a medieval blacksmith a large bellows provided the forced draft needed for a hot fire. The bellows was most efficient if one opened it fully before closing it and opened it quickly and closed it slowly. The blacksmith learned to operate the bellows in this way because of the reinforcing result of a steady, hot fire. He could have learned to do so without describing his behavior, but a description may have been helpful in operating the bellows properly or in remembering how to do so after an interval. A short verse served this function: "Up high, down low, up quick, down slow, and that's the way to blow." (...) the blacksmith's (behavior) was both contingency-shaped and to some extent rule-governed after he had discovered the rule. (Skinner, 1974, p. 108)

Here Skinner is describing that contingency-shaped behavior and rule-governed behavior are different learning processes, with different advantages; however, they can be 
complementary in a way where one makes the other stronger. More precisely, Skinner (1974) suggests that important changes are achieved in behavior when it is shaped by contingencies, and these changes can be nuanced and refined, not achievable though verbal description, simply because the subtleties are difficult to verbally describe. This kind of change in important and frequently subtle aspects of the client's repertoire is what the FAP therapist aims to achieve experientially through direct shaping in the therapeutic relationship.

In contrast, if rule-governed behavior is useful for verbally describing similarities between contingencies (Skinner, 1974), accurate rules can facilitate the extension of changes to other contexts by making it easier to repeat a successful behavior in a different, but similar, context. Thus, the therapist tries to improve the accuracy of a client's descriptions of his or her own behavior during the analytic part of FAP, clarifying the similarities between different contexts and enhancing the chance that a response that was shaped during the experiential part of FAP will occur out of session.

\section{The Potential Importance of the Analytic Component of FAP}

The central goal of FAP is to shape CRB2s through natural reinforcement in session, and this behavior change in session should extend to other functionally and topographically similar environments as a natural result, without invoking verbal relations. Ideally, in FAP several stimulus and response classes with a variety of topographies are shaped to help the responses occur in a variety of contexts, increasing the likelihood that the response will meet naturally occurring reinforcement contingencies outside of therapy. FAP employs traditional behavior shaping procedures, not unlike what one would find in other applied behavior analytic contexts, such as managing classroom behavior, working with a child diagnosed with autism, or even training non-human animals (e.g., Pryor, 1984). An argument could be made, in fact, that this primary mechanism in FAP does not require a verbal analysis at all, and can be interpreted completely in terms of contingencies.

However, the transposition of in-session learning to other environments occurs not only by a stimulus generalization process. In fact, Johnston (1979) suggests that researchers and practitioners should not use the term generalization to describe the process of extending behavior to other contexts at all, because the term technically refers simply to stimulus or response generalization, and the process of extending behavior to the natural environment is complex and requires additional behavioral principles for a full understanding. The analytic part of the FAP interaction thus invokes a variety of behavioral processes, most of them verbally mediated, to achieve the desired outcomes. These will be discussed in detail below. 
It is important to remember that even from a traditional behavioral perspective there may be problems with generalizing the occurrence of the behavior to other contexts. Specifically, while problem behavior is relatively easily evoked and effectively responded to in therapy, improved behavior is not necessarily easily evoked and reinforced in daily life. The occurrence of problem behavior from daily life to therapy as therapy begins is likely because the problem behavior is of high strength (otherwise, the client would not be seeking help to change the behavior) and sufficient functional similarities exist between the context of daily life and therapy. On the other hand, the contingencies that evoke the problem behavior outside therapy may have been in place for many years, making behavior change unnatural in the daily life context, and thereby making the transfer of session improvements to outside of session more unlikely than the transfer of problem behaviors in to the session. Johnston (1979) discusses that when a behavioral change occurs it means that appropriate contingencies were present, at that moment in the environment, but it does not mean that the behavior is changed in such a way that it will occur in other contexts. In the FAP context, changes occur because new responses are evoked and receive proper consequences by the therapist, but on a daily basis, sudden environmental changes to evoke new and more appropriate responses will hardly occur, making it essential that the therapist plans strategies to evoke responses out of session to produce reinforcement in this new context. Again, many of these strategies will occur during the analytic portion of the therapy.

In addition, newly reinforced responses (CRB2s) may still be at a lower overall strength compared to $\mathrm{CRB} 1$ alternatives and their longer reinforcement history, making CRB1s more probable. As behaviors are multiply determined, the in session situation that evokes the CRB2 will not be completely the same as the out-of-session situation. The client needs to emit the new behavior in the presence of other stimuli (not common to the therapy room) that have a history of strongly occasioning the CRB1 response class. A verbal formulation that disrupts these other sources of stimulus control may be required to supplement any partial stimulus generalization that might be in place. Based on this interpretation, FAP's mechanism of action (therapist contingent responding to $\mathrm{CRB}$ ) may turn out to be necessary, but not sufficient.

There exists research about the mechanisms of change in FAP that shows that conducting FAP therapy leads to changes in out of session behavior. Callaghan et al. (2003) and Busch et al. (2010) made efforts to collect data about outside therapy target behaviors, analyzing how frequent the client mentions the occurrence of outside problem or improved behaviors during the therapy session. The findings of these studies are not robust, suggesting that there exists an opportunity measure these behaviors at least in this way, but more efforts 
need to occur in this area. Other research has been conducted with the goal of collecting more direct data about outside improvements as a result of FAP interventions using client diary cards. Kanter et al. (2006), Landes et al. (2013) and Lizarazo, Muñoz-Martínez, Santos e Kanter (in press) showed that improvements were observed in most of cases. However, none of these works manipulated the analytic part of FAP to demonstrate the responsible mechanism for the outside improvement, confirming whether the analytic part leads to generalization or if the experiential part is sufficient to cause this change in behavior.

Further, it may be possible in some cases that verbal descriptions of the contingencies and verbally established parallels between therapy and outside contexts may be necessary, not simply supplementary. For example, the client might generate a rule that therapy is the only safe place to express irritation, which (if not explored with the therapist) could actually inhibit occurrence of this behavior in other environments. A functional analysis could help the client to recognize other contexts that are safe like therapy, facilitating the transfer of the learning process.

Although there is some evidence that FAP may lead to generalization from in session to out of session behavior, there is no evidence about which FAP mechanism is responsible for that process. Thus, without more evidence determining the relevant mechanisms, it is reasonable to hypothesize that combining the experiential and analytical aspects of FAP may maximize the efficiency and efficacy of the interaction, directly shaping changes in the client's repertoire inside the session but also increasing the chances that these changes occur outside therapy by clarifying, describing, and otherwise analyzing the contingencies experienced in session. This is discussed in detail below.

\section{Definitions of and Relations between Rule 5 and CRB3s}

Rule 5 and CRB3s may be seen as providing rules and promoting rule-governed behavior in clients. Skinner (1966) described rules as verbal descriptions of contingencies and rulegoverned behavior as behavior controlled by descriptions of contingencies rather than the contingencies themselves. More recently, rule-governed behavior has been defined as any behavior controlled by verbal antecedents (Hayes, Barnes-Holmes, \& Roche, 2001; Törneke, Luciano, \& Valdivia Salas, 2008). These recent definitions were not considered by Kohlenberg and Tsai (1991), who appropriated the original terms from traditional behavior analysis for use in FAP. Thus, although much behavior that occurs in therapy or in relation to what occus there may be seen as at least partially under the control of verbal antecedents, the more restrictive 
and traditional definition of rules, specifically as descriptions of contingencies, has been employed in FAP.

Originally Rule 5 was described as any descriptions given by the therapist to the client of contingencies affecting the client's life (Kohlenberg \& Tsai, 1991). Similarly, CRB3s were defined as descriptions of essentially the same contingencies but made by the client. Thus, one could say that the content of the descriptions for both is similar, but in one case the description is provided by the therapist (Rule 5) and in the other it is provided by the client (CRB3). However, it is still unclear exactly what a good description of a contingency is in FAP terms.

Ideally, a rule as described by Skinner (1966) implies a complete specification of the three-term contingency describing the response, its antecedents, and its consequences. Schlinger and Blakely (1987), however, mention that a combination of two of these three elements would already be considered a rule. In actual clinical practice, it is common that one of the elements of the analysis is missing in either Rule 5 or CRB3s provided by the client. A few logical questions that follows are: Would all three elements of the operant response be required for a response to be considered a Rule 5 response or a CRB3 in FAP? Which of these three elements are most important to be described to promote changes in or out therapy?

While there remains no empirical evidence to guide us, one may say that it seems helpful that the description includes the antecedent. Without the antecedent in a description, the transfer of in session improvements might be imprecise or inappropriate, but it seems essential that Rule 5 and CRB3s include at least the a specification of the behavior and its consequence. In a functional analysis, the consequence of a response is the variable responsible for maintaining it; thus, describing the consequence seems to be essential for saying that the analysis is indeed functional and helpful for changing a response. Hypothetical examples of such descriptions can be seen below:

T: It seems that when you say to her something like that, although she gets angry, you still benefit a bit, because she ultimately tends to do what you want, right?" (Rule 5 about what happened outside the therapy.)

C: Yes. She fights with me and gets angry, and that makes me want to stop talking to her. If I can stay in there with her, even when I am stressed out, she often comes around and gives me what I want. That really makes me feel better. (CRB3 about what happened outside the therapy.) 
T: I feel like when I disagree with you, you get very irritated with me, and it becomes really hard for me to be honest with you. (Rule 5 about what is happening inside the therapy.)

C: The problem is, when you disagree with me, I feel you think I'm doing everything wrong - like you're showing me all my mistakes, and that makes me feel badly. I think that's when I get mad, well, like you said, irritated. It is just a little easier to deal with being irritated at you then admitting I did something wrong. (CRB3 about what is happening inside the therapy.)

T: Although normally you would be irritated with what I said, you acted differently right now and weren't aggressive with me. I'm really glad you did that. It made it much easier for me to stay honest with you. I think that if you could act this way also with your brother, the relationship could get better, you know, more connected for you with him. (Rule 5 about what is happening inside the therapy including a parallel with outside the therapy.)

C: The problem is that it's ok not to be aggressive with you, because I know you won't be aggressive with me, but with my brother, it's different. I think he'll be aggressive anyway. (CRB3 including a parallel between what is happening inside the therapy and outside the therapy.)

As the above examples illustrate, a second issue related to the definition of Rule 5 and CRB3 responses concerns whether the analysis focuses on in-session or daily-life content. The first example focused on daily-life content, the second on in-session content, and the third paralleled daily-life with in-session content. The original descriptions of Rule 5 and CRB3s (Kohlenberg \& Tsai, 1991) allowed for any analyses to be considered here. However, the hypothesis in FAP is that the best analyses to maximize the transfer of in-session improvements to outside of therapy are those that focus specifically on in-session content. Moreover, a focus of those descriptions that parallel in-session and daily-life content, instead of analyses that focus only on daily-life content., are more salient to the client and will more likely generalize out. Stated differently, the therapist maintains a focus on in-session behaviors (and parallels that occur to outside session) and helps generate descriptions of which client behaviors are more effective, rather than simply discussing events that only occur outside of session. The assumption behind this hypothesis is that Rule 5 analyses and CRB3s in FAP should be strongly related to the process of reinforcing $\mathrm{CRB} 2 \mathrm{~s}$ that occurred in the therapeutic relationship, 
focusing on the parallels between events in this relationship and the client's daily life relationships. In other words, when there is an improvement in session (CRB2), the description of the relevant contingencies, formulated by either client or therapist, should clarify the contingencies just experienced. Clear descriptions of contingencies experienced in session that were successful, in turn, should better control the client's behavior, increasing the chance of change outside therapy.

The use of the term CRB3 can be confusing, because CRB1s and CRB2s are distinctly focused on in-session content, but the CRB3 content may vary. As discussed in Kohlenberg and Tsai (1991), the logic of CRB3 is that any description of his or her own behavior provided by the client should be attended to and shaped by the therapist. This means that even descriptions of speculative relations between events that are not actually linked by contingencies should be considered as CRB3s (or approximations) because they are opportunities to shape more functional descriptions. Likewise, descriptions of out-of-session contingencies without parallels to in-session contingencies are seen as useful opportunities for shaping functional descriptions but are less related to FAP's specific hypothesized mechanism of action because they do not incorporate the in vivo experiences of therapy and will be given less attention in the following discussion.

\section{Promoting Effective Rules}

The behavior analysis literature contains considerable discussion and empirical study of rule-governed behavior (Hayes, Kohlenberg \& Melancon, 1989; Hayes, Zettle \& Rosenfarb, 1989; Meyer, 2005; Skinner, 1974; Törneke et al., 2008). This literature provides some guidance for understanding how Rule 5 and CRB3s can both promote effective rules about the therapeutic and other relationships and increase the probability of transferring behavior change to conditions outside therapy.

Rules can be about how the client behaves in specific situations, promoting selfknowledge, or about how the client could behave in terms of goals and recommendations to be followed, promoting self-control. Tourinho (2006) pointed out that self-knowledge involves privately discriminating stimuli and responses, suggesting that self-knowledge is selfdiscrimination. Skinner (1969) also said that self-knowledge is the description of one's own "behavior and the contingencies responsible for its strength" (p. 193-194) and that self-control depends upon self-knowledge because the person becomes more able to manipulate relevant variables, changing his or her own behavior (Skinner, 1953). As Tourinho (2006) pointed out "self-control implies self-knowledge" (p. 67), because discriminating variables is important to 
change them. However, the author also pointed that self-knowledge does not necessarily lead to self-control. Therefore it seems relevant to discuss strategies that can increase the probability that self-knowledge will have an influence on self-control. The question becomes, can analyses about the therapeutic relationship, based on interactions that just occurred between client and therapist, help the client analyze his or her own behavior (self-knowledge) in other contexts in a way that recognizes contingencies more clearly, increasing the chance of changing the behavior (self-control)?

A client's behavior should be under the control of effective contingencies, not therapist approval

As discussed, rules generated in therapy may be important in the development of selfcontrol. However, not every rule effectively enhances self-control. As described by Hayes et al. (1989), there are at least three different kinds of rule-governed behavior-pliance, tracking, and augmenting - and each kind of rule-governed behavior implies differential forms of control.

Pliance is a form of rule-governed behavior that occurs when the reinforcement for the individual to follow the rule is social reinforcement for rule-following behavior. In the context of therapy, pliance occurs when the client follows the rule in the context of the therapist providing reinforcers for following that rule, such as providing approval. This rule may have been generated by the client as a CRB3 or instructed by the therapist. The client then complies with the rule because praise he has received before for following rules (which functions as a reinforcer), rather than adhering to the rule because it is similar to the contingencies observed and would be helpful to improve his or her interactions with others. This type of behavior, which is emitted under the control of someone's approval, runs counter to the long-term goals of FAP (although it may be useful in the short-term).

The objective of the therapist (in most cases) is not to have an obedient client, but to have a client who behaves effectively in life with respect to his or her own goals and values for living; that is, the client lives a richer, vital life as he or she defines it, not to please the therapist. In this sense, tracking (as opposed to pliance) is a more useful form of rule-governed behavior. With tracking, behavior comes under control of the apparent correspondence between the rule and the contingencies described by the rule (Hayes et al., 1989). In other words, the rule is followed because it effectively puts the rule-follower in contact with contingencies. In this case, the approval of the therapist does not maintain rule-following because the tracking behavior is influenced primarily by natural and direct consequences of following the rule out of the clinical session. Here, the therapist may help shape a CRB3 by reminding the client that 
he or she needs to continue to notice the effect of his or her behavior on others or the situation. Moreover, the point is to follow broader rules that help the client improve his or her life and remind the client to be flexible to changes and shift in context outside of therapy. For example, consider this interchange:

C: So, I think I get it. When I get really upset, and I close down with you, when I pull back and stop talking, it makes it harder for you to actually help me out. You feel disconnected from me and can't really help me. I think then I get way more upset. But when I tell you "I need help and I am scared, you really do lean in, as you say, and you actually do help me out. I feel much more calm then. (CRB3 for in-session problems and improvement)

T: Yeah, I think you really get it. This is great. It really feels like you have a got a handle on what happens in that situation here.

C: Yeah. So, I need to do that more outside. I need to tell people when I get upset that I am scared and I need help.

T: Well, yes. And no. I mean what you say to other people will depend a great deal on who it is, what is happening, and really, whether you saying it that way actually helps ease your pain and gets them on board with you. Does that make sense? I am saying that the overall goal is to have you trying things a lot of different ways. You found a way that works with me to help you get what you need. When you try that with others, how you do it will likely have to change. And, more importantly, you need to see if that works for you, and then adjust it, try it differently, so you get your needs met.

The third kind of rule described by Hayes et al. (1989) is augmenting, in which the rule emphasizes contingencies that the individual already has contacted, but changes the future reinforcing or punishing value of the contingencies. In some cases, these rules are considered as motivative augmentals, because they temporarily change the value of reinforcing and punishing stimuli; in other cases, they are considered formative augmentals because they establish new stimuli as reinforcers or punishers (Hayes et al., 2001). In both cases, the augmental is a kind of rule that can alter the functions of future response consequences.

It is possible that much therapeutic talk in general serves augmenting functions, but such an analysis is beyond the scope of this paper. Here, we are interested specifically in how therapists may augment functions of responses that occur in the therapy relationship. For example, a therapist may say to a client, "I know that your crying in here is embarrassing to you, because you've been ridiculed in the past for showing emotions, but I want you to know that when you cry in here with me, I am looking at you the way I am right now because I see 
you as having great courage and strength." Here, the therapist is providing a verbal description that may, if effective, alter the functions of affective expression and eye contact from someone when expressing difficult feelings so that these behaviors are more reinforcing and less punishing.

To summarize, in FAP it seems helpful to create in-session rules that function as tracks and augmentals, which may help the client to change his or her behavior out of session. However, as Hayes et al. (1989) point out, rules function as plies, tracks, or augmentals as they play out in the life of the rule-follower, not because of the intentions of the rule-maker. The rule provider, in this case the therapist, cannot always build the rule in the intended way. Perhaps, however, there are ways to develop rules that are more likely to serve as tracks and augmentals for the client.

The hypothesis here is that rules created in response to interactions that have already happened between the client and therapist should be helpful to promote more effective behaviors outside of session. If the rule is formulated based on interactions experienced by the client as being positive, it may achieve tracking and augmental functions rather than only pliance in the therapy relationship. In this case, the interaction experienced in session may make the rule more likely to be followed. The rule, then, would make the behavior emitted in session more likely to occur both in and out of session by clarifying the contingencies related to the response. Abreu et al. (2012) stipulated that by describing the relationship between the therapist and client new rules will be shaped that can serve as tracking rules for clients. Further, these rules can be helpful because they describe functional equivalencies between what occurs in and out of session.

It might be useful to both explicitly shape and point out to a client when pliance, tracking, or augmenting occur (though the therapist may choose not to use those terms with the client). For example, a client may do something helpful toward his or her goals, but it may be unclear why or under what control the client's behavior occurs. The therapist may ask, "Did you do that because we talked about it in session and I suggested you should try, or because you thought it might be helpful based on the goals we are working toward in therapy?" Note that the client doing something for the therapist that serves the client's goals but was because the therapist suggested it may not be the worst thing to occur. In fact, in therapy, it may be an early approximation for the client to begin to move toward more value driven behavior by complying with a suggestion in order to develop a broader repertoire. For example, if a client does not know how to engage a task, the therapist might suggest, "Try it this way," and the client might 
comply. The goal, though, is to develop more tracking behavior over time even if short-term compliance is useful.

The therapist should improve the client's ability to analyze his or her own behavior

As discussed above, clarification of the contingencies involving the client's behavior in session can help change the behavior out-of-session. However, if the therapist is the only one in session who understands and is able to formulate accurate descriptions of these contingencies (i.e., by regularly providing Rule 5 interventions), much less impact on the client is expected than if the client learns to make these descriptions him or herself (i.e., by shaping the client's CRB3 repertoire). Catania (1998) and Wechsler and Amaral (2009) analyzed research on the correspondence between verbal and non-verbal behavior and concluded that shaping verbal behavior may lead to changes in non-verbal behavior more easily than simply giving instructions or even the direct shaping of the non-verbal behavior. In other words, it seems important that the individual learn how to describe his or her own behavior to more easily change non-verbal behavior. Bring this idea into the context of doing FAP, it is probably important that the client not only hears and agrees with the therapist's Rule 5 analyses in a passive way, but also learns through therapy how to conduct effective analyses him or herself. In this sense, it is important that the therapist focuses on shaping CRB3s instead of primarily focusing on Rule 5 in which the analysis is provided for the client. The client needs to be able to generate the analysis of their behavior and track this. Perhaps Rule 5 should be defined as more than the therapist talking about client's behavior, but also as shaping the client's CRB3 analyses, because ultimately the goal of functionally extending behavior to out-of-session contexts (the point of Rule 5) may be improved.

When considering the importance of shaping the client's CRB3 repertoire in session, one might consider the CRB3 in this instance to be a CRB2, an effective client behavior to be reinforced. However, it may remain useful to continue to consider such behavior as a CRB3 rather than a CRB2. The definition of CRB1s and CRB2s involves an idiographic case conceptualization of the client's goals for psychotherapy (Tsai et. al., 2009), so CRB1s and CRB2s are defined as the client's problems and improvements in daily life that appear in the session and are a target for intervention. In general, the lack of effective functional analysis of one's own behavior is not considered a problem in a client's life, but it is considered as a repertoire that can help with the development of CRB2s. Thus, as quoted above, Skinner (1974) remarked how a short verse about the blacksmith's behavior helped him in his daily work, but was not essential for the execution of the behavior. 
The client's rule-governed behavior should be sensitive to changes in contingencies

Some research has suggested that rules can lead to insensitivity to changes in the contingencies (see Hayes, et al., 1989 and Abreu-Rodrigues \& Sanabio-Heck, 2004, for reviews), which can produce stereotypical rule governed behavior in which one continues to follow a rule even if the contingencies have changed. As reviewed by Abreu-Rodrigues and Sanabio-Heck (2004), several contextual features may decrease the chance that this kind of insensitivity will occur and may increase contact with actual contingencies: (1) contact with discrepancies that exist between rules and the contingencies that were the bases for the rules; (2) rules that describe the behavior in vague or non-specific ways; (3) contact with a variety of rules describing different situations; (4) decreased reinforcement for rule-following behavior; (5) weaker histories of reinforcement for rule-following; and (6) stronger repertoires for discriminating present contingencies.

Several implications stem from this review. First, in a clinical situation, when rules are generated from analyses of the client's out-of-session behavior, the usefulness of the rule that is generated depends in part on the accuracy of the client's description of the situation. On the other hand, when a rule is created based on an interaction between the therapist and the client, they both have observed the contingent relations existing between them, decreasing the possibility of discrepancies between the created rule and the lived contingencies.

Second, the therapist should help the client analyze what happens in session functionally not topographically, describing strategies for responding (e.g., staying connected, opening up) rather than specific topographies (e.g., saying "I am scared"), and relating this with the consequences. This recommendation is consistent with research by Bicard and Neef (2002) who found instructions that identified strategies were more sensitive to changes in contingencies than instructions that specified topographies of behavior. The goal is for the rule to apply to a large class of responses and not to a specific response, enhancing the chance that a single rule applies and can be adapted to different situations.

Third, ideally, rules are created and revised over several therapeutic interactions and subtleties in the interactions and discrepancies between the rule and actual life experience. These can be discussed as the rule is revised in an effort to increase the probability that the client will follow the rules in a less rigid and more flexible way in order to remain sensitive to discrepancies between the rule and experience. Finally, it seems important that the client acquires of repertoire of rule formulation him or herself as discussed above, to increase the client's capacity to identify functional relations between events, allowing the client to change the rule in the presence of a change of contingencies. 
It is essential to note that even in the client-therapist relationship, there are idiosyncrasies that may occur with the two people involved that do not occur or function in the same way outside of session. It is again useful for the therapist to observe and help shape this discrimination skill with the client. For example, this type of interaction may occur:

T: I noticed you just tried something really neat with me. You were able to open up when you were noticing you started to close yourself off. You even said that to me, "I am noticing I want to shut down," and then you really shared what you were struggling with, and I think I really understood that. Does that feel right?

C: Yeah, I was able to hang in there with you.

$\mathrm{T}$ : Yeah, and did you feel heard or understood by me?

C: Mmm hmm, I did.

T: That's really great. I am really glad in lots of ways. Especially because it's what you have been working on in therapy, really staying connected when you get upset.

C: Thanks, yeah.

T: OK, how do you think that what you just did was because of me, or this relationship?

Do you think you could do this with other people, like your partner, outside of here?

C: It's really safe here. But, I don't know if I could, why?

T: Because what you and I do is very important, and it matters to me a great deal, but what you do outside, that you can do this with others, that's the most important thing in our work here. We need to think about what allowed you to do that in here with me, and how you could do that with other people. And really, why or when you would, how you would know it is safe, and how you might stay connected with others in moments like this with people who are not me. That's our goal together. That's the bigger picture of all of this work we do.

In this way, the therapist helps the client discriminate and respond functionally, not only to the therapist in this context (a session), but the therapist is also helping the client contact his or her value (i.e., a specific reinforcer in a relationship) and the rule that clarifies the contingencies involved in it. The rule, in this example, will be described as identifying a client's value and ways to reach it. The client trying a newer, more challenging behavior (remaining present with difficult affect rather than escaping) will need to occur outside of therapy. The therapist will need to encourage that behavior to occur in a variety of forms. The client will need to discriminate different situations and people and also different behaviors that might allow the client to accomplish this goal, then engage in a variety of responses aimed at reaching the goal, and finally noticing the impact of his or her own behavior. 


\section{The client must be able to emit the new behavior at the appropriate moment}

As Tourinho (2006) and Meyer (2004, 2005) pointed out, even considering rule following behavior, it is necessary to remember that the simple description of the contingencies controlling our own behavior are not enough to actually change the behavior. As it was already mentioned, Skinner (1969) and Tourinho (2006) declared that self-control depends upon selfknowledge (descriptions of the contingencies of control), but the opposite is not necessarily true. An important part of the process is for these descriptions to have an influence over the stimuli that control the client's behavior (Meyer, 2000), leading to rule-following behavior; in other words, the rule made in session should result in transformations of relevant stimulus functions. As Schlinger and Blakely (1987) point out, rules can be considered as contingencyspecifying stimuli and as function-altering stimuli. Considering these function-altering characteristics that rules may have, the degree to which there is functional similarity between the therapy context in which the rule was developed and relevant daily life contexts is important in determining the probability that the description of the events occurring in the therapeutic relationship will change the functions of the relevant stimuli in the client's daily life.

As illustrated in the example above, the idea is that a rule about a process that occurred in the therapeutic relationship can be created based on an experienced interaction, hopefully leading to tracking. This rule can be related to other functionally similar situations that happen out of therapy, augmenting and altering the functions of daily life stimuli, helping the client to emit the new behavior in contexts that normally evoked ineffective or problematic behavior. In this case, rules based on the contingencies experienced by the therapist and client in session would change the stimulus control that resulted from the direct in-session shaping process. It should be noted that there are other concepts in the literature that deal with these transformations of function, including establishing operations (Michael, 1982, 1993) and supplementary stimulation (Skinner, 1957, discussed by Meyer, 2000), apart from the afore mentioned augmentals and relational transformations of function in terms of Relational Frame Theory (Hayes et al., 2001).

\section{Suggestions for Clinical Implementation of Rule 5 and Shaping of CRB3}

In summary, the analytic portion of a FAP session helps the client contact the existing contingencies that occur during the experiential part of the session, clarifying and increasing the client's sensitivity to them. In this way, the analytic aspect of FAP could make it more likely that CRB2s emitted during the experiential part of the session will be repeated out of the session achieving the goal of the intervention. At the same time, the experiential part of the 
FAP session serves as the repository that allows the therapist and client to clarify, refine, and make more effective the rules that were formulated during the analytic portion so that the experiential and analytic parts of the FAP session have a transactional relationship that evolves over time. Ideally, through this interplay, during the experiential part of the FAP session the stimuli which originally evoked CRB1s will increasingly evoke CRB2s, and over time, CRB3s as well. These CRB3s, when evoked in the client's daily life, will function as effective rules for the engaging in improved behaviors (parallel to in-session CRB2s) in appropriate contexts.

This review has discussed several issues to consider how to maximize the effectiveness of the analytic part of FAP. First, descriptions of contingencies should help clients discriminate subtle, private controlling variables that may enhance self-knowledge and lead to more refined self-control.

Second, when providing analyses, therapists should try to include a full description of aspects of the three-term contingency - the antecedent, behavior, and consequence. When shaping CRB3s in clients, any description (e.g., of an antecedent and a behavior) may be considered appropriate initially, and over time successive approximations to descriptions of all three terms should be shaped. In this way, the CRB3 repertoire is treated similarly to CRB2 repertoires in FAP.

Third, when providing rules in FAP and encouraging rule-governed behavior, therapists should be sensitive to pliance and attempt to maximize tracking and augmenting to reduce the likelihood of overly rule-governed behavior that is under the control of pleasing the therapist rather than the natural contingencies that will support the behavior outside of session. Ways to minimize this possibility include defining rules functionally, not topographically, so that a single rule can apply to multiple situations. Creating and revising rules over several interactions and discussing discrepancies between the rule and experience may help the client remain sensitive to such discrepancies in his or her daily life. In addition, the therapist can help develop descriptions from specific interactions that have occurred between the client and therapist, ideally recently, such that a connection between the experience of the contingency and the description is salient to the client and then discussing what would be similar and different when the client tries this outside of session. The therapist then consistently prompts and encourages attempts by the client to try these behaviors repeatedly both in and out of session in the service of building tracking and possibly even augmenting behaviors.

Fourth, parallels between in-session and out-of-session antecedents should be made to increase the probability that the rule will be evoked and control behavior in the appropriate outof-session contexts. Fifth, therapists may provide Rule 5 analyses but should focus on shaping 
CRB3s such that the client will learn to conduct the functional analyses of his or her own behavior.

\section{Clinical Example}

A modified clinical example is provided to exemplify how the ideas described above may facilitate a client's progress.

Brad is a young adult who is very competent in his job. He started working at a large company several years ago and has received consistent compliments and praise from his boss about the quality of his work. However, after a few years in this position, he became disappointed, having been overlooked for promotion several times in favor of others less qualified than him. Following the advice of his sibling, he started therapy. Brad and the therapist quickly analyzed the situation. Apparently, although very competent, Brad was an unpleasant colleague.

The therapist and Brad discussed situations when Brad received a compliment and acted very arrogantly toward his colleagues and his boss in response. The therapist tried gently to discuss with Brad how people were pulling away from him in response to his behavior but Brad defended himself with the notion that work was not a place to have friends, and because of that he did not care about his colleagues' distance. He was able, however, to entertain the idea that he was not being promoted as a consequence of his behavior rather than the quality of his work.

Brad was a challenging client as well. During therapy, he frequently acted as if the therapist's comments were obvious and not important. After discussing the rationale for FAP with Brad, they agreed to try FAP, although Brad reported that he believed that it was not going to be helpful. During a session, Brad reported an interaction with colleagues at work. When the therapist tried to point out to Brad how his reactions may have pushed his colleagues away, Brad rejected the therapist's view. The therapist asked if the way Brad felt in that moment was similar to the way he normally felt when he was responding arrogantly to the colleagues (Rule 1 - watching for CRBs checking the out-to-in parallel). Brad took some time to understand the question, but when he did, he admitted that the feeling was similar. The therapist then asked more about the kinds of feelings Brad was having in the moment (Rule 2 - evoking CRBs) and after some time Brad admitted that at that moment, he was feeling really insecure. Specifically, in that moment, he thought: "Yes, my therapist is right... how stupid that I didn't realize it before." The therapist considered Brad's response to be an approximation to a CRB2, because he was being honest expressing his insecurity and displaying some vulnerability by admitting that he did not have all the answers. The therapist responded that although she really understood 
how difficult and uncomfortable it was for Brad to feel and express the way he did, it was much easier for her to understand Brad and helped her want to continue working with him to understand his relationships in this moment (Rule 3 - reinforcing CRB2).

This interaction would be considered the experiential component of FAP, in which new behaviors are being shaped through the therapeutic relationship. Similar interactions occurred over the next several sessions, and the therapist observed that over time it became easier to evoke Brad's CRB2s, including demonstrating more genuine interest in the therapist's point of view, suggesting that she was responding to him in a way that was reinforcing (Rule 4 checking the reinforcing effect of therapist behavior). The therapist started to emphasize strategies to transfer the in-session improvements to outside-of-session, as in the following interaction that occurred several weeks into this work. Aspects of the interaction that exemplify the key points of the discussion will be described.

Therapist: Brad, over the past several sessions, I have been pointing out to you how I feel in response to when you behave dismissively in here. Sometimes it shows up as dismissiveness, other times it shows up as arrogance, and other times simply you seem bored by what I am saying. Regardless of what it looks like, it seems to be triggered by when you are feeling silly or stupid, and you don't like how that feels. (Here the therapist is describing the antecedent and the behavior of an operant based on in-session experiences shared by the client and the therapist. Also, notice that the antecedent is a subtle, private event, that of "feeling silly or stupid." Helping Brad discriminate these subtle antecedents may enhance self-knowledge and self-control). Do you see what I am talking about?

Brad: Yes I do. We've talked about this several times.

T: Right. Last time I think we talked about how when you felt confused, you made it seem like you were bored. (The therapist is referring to the history in which this rule has been clarified and revised over time, to help keep Brad sensitive to the actual experience, i.e., tracking, and not become stereotypical in application of the rule.) How would you describe what we're seeing now? (Here the therapist is prompting Brad to engage in CRB3 himself rather than relying on the therapist's analysis.)

B: Well, sometimes when I feel stupid, I get defensive.

T: Right. I think that is a good way to put it. But sometimes you feel stupid, sometimes confused, sometimes silly. I wonder if a word that captures all that and feels right for you is 'vulnerable'? (The therapist is formulating the rule turning more salient another broad 
aspect of the situation, probably less aversive. That way, the therapist is formulating an augmental rule.)

B: I suppose that feels about right.

T: And sometimes how you get defensive looks bored or arrogant or irritated, but underneath it really is about being defensive? (The therapist is continuing to formulate the rule in the client's language, describing again a broad aspect of the situation, formulating another augmental rule.)

B: Right.

T: And when that happens, how do I respond?

B: Sometimes you do nothing and just let it go, but sometimes you'll point out to me that you don't like it when I do that.

T: Right. And why don't I like it? (The therapist is sensitive to pliance here.)

B: You say it makes it harder for you to understand me and help me.

T: Yes, I think that is about right. Does that fit your experience, or do you feel more that that is just the right thing to say in here to me? (Again, the therapist is sensitive to pliance, and is asking questions to maximize tracking instead.)

B: No, it definitely fits my experience.

T: Ok, and the thing I really want you to pay attention to, is when you're NOT like that, when you are more open and in touch with feeling vulnerable, how do I respond?

B: You say it helps you understand me, and you say it makes you want to help me more.

T: Right, it is like you are more human to me, so I feel more caring for you. When you let me know that sometimes when you feel silly or stupid, vulnerable, you react defensively, it makes it easier for us to talk and find ways for me to help you. You have been doing a lot more of this lately, and I feel I'm able to help you more, increasing my willingness and even my efforts to do so. I feel good about being your therapist especially at times like this. (The therapist here is clarifying the three term contingency, including the antecedent of his private controlling variables and his behavior but is focusing on clarifying the consequence for him, which is the most important component for him to learn.) How would you put it, in your words? (The therapist is focusing on shaping his CRB3.)

B: I would just say that you respect me.

T: Right, I definitely feel more respect for you. (The therapist is attempting to reinforce his $C R B 3$.) So, the question is, do you think that something similar could happen in your work environment? (The therapist is drawing a parallel with the out-of-session context.) 
B: Maybe... you are suggesting that the way I talk with my colleagues and even my boss is similar to what is happening between us?

T: Yes, this is what I'm suggesting. How do you normally feel and act at work when you receive a compliment from your boss?

B: Well, I normally feel insecure at my work... even when my boss says I did a good job, I feel it could have been better, and I feel relief at the same time because he did not see all the mistakes I did... Somehow I try to emphasize the compliment, to make it more difficult for him and my colleagues to see my mistakes. Ok... probably this is really annoying to them and I'm pushing them all away, including my boss. (Brad is engaging in a nice CRB3 here.)

T: Exactly! This all makes sense to me. You are protecting yourself, but it has this real cost to you. Maybe you could try something different, maybe not talking openly about your insecurities because that is more what you do with a therapist, not a work colleague, but trying not to pretend all the time that you are so secure. (The therapist is helping Brad see subtleties in the interaction and discriminate differences between therapy and work to clarify the rule.)

B: I see. Maybe if I do that, they will not push back at me all the time, including my boss.

T: Yes. I think that's really important to notice. You see the differences between here and there, and that you may need to really try a lot of different approaches to get what you need, to be much more effective with other people, like your boss.

Interactions like this are considered the analytical component of FAP, in which the therapist helps the client to understand the improvements made in session, making parallels with his daily life, and shaping CRB3s. The hypotheses is that these parallels, especially one made by the client, will help the client not only behave more effectively in this particular situation but also learn how to analyze his behavior and create effective rules that accurately track the contingencies in other situations. These rules, added to the experiences of direct shaping in session, would supplement the client's behavior, helping him to behave more effectively out of session.

In Brad's case, the analyses of Brad's outside behaviors at the beginning of the therapy were not sufficient to change his behavior at his work environment. At the same time, the improvements achieved in session (CRB2s) were not sufficient either. The integration, however, of both may be a more powerful mechanism to produce the necessary changes at the important moments, because the client has already experienced such changes at the appropriate moments in session. These new behaviors out of session would hopefully be reinforced and 
would increase in frequency, demonstrating the transfer of CRB2s to daily life, not because of stimulus control related to therapist approval, but because the client is obtaining more outside reinforcement for the improvements. At the same time, because these new rules are based on situations experienced by the client in session, it may be easier for him to learn how to analyze new situations and change the rules if the circumstances change.

\section{$\underline{\text { Summary and Future Directions }}$}

This paper provided a theoretical investigation of the analytic aspects of FAP and is meant to supplement the larger theoretical literature on the experiential aspects of FAP. Many of the issues discussed in this paper are speculative and necessitate empirical investigation. Our hope is that the analysis provided in this paper will help support and stimulate future research, allowing for a better understanding of the analytic component in FAP, and maximizing the effectiveness of FAP-based interventions.

Both large scale randomized trials and additional single-subject research is necessary for FAP. Large scale randomized trials may best define FAP as a package that includes Rules 1 through 5, and we are hopeful that the current clarification of Rule 5 will benefit explorations of FAP as such a package. In addition, smaller scale, single-subject research may be able to explore the specific benefits of components of this larger package. For example, while we have attempted to build a logical argument for why the analytic aspects of FAP are important, research can test FAP with and without this aspect included in single-subject designs. Such research could also occur in basic laboratory settings. For example, using the established "Fast Friends" procedure (Aron et al., 1997), a participant could interact with a trained experimenter in the lab, in which the experimenter attempts to reinforce vulnerable self-disclosures after a personal question. This procedure could include be manipulated to either include or not include an analytic Rule 5 component in which the experimenter also describes the interaction, and the effects on generalization of self-disclosure to others could be evaluated. Such a design is one example of how the experiential and analytic aspects of FAP could be studied in a controlled setting.

In general, this paper emphasizes analyses of the interchange between the client and the therapist in FAP at the level of verbal behavior (e.g., rule-governed behavior and relational framing). Much more can be discussed and researched with respect to verbal behavior in FAP. It is possible, for example, that the description of the functional analysis may clarify and suggest additional interventions for FAP therapists to try with respect to Rule 3 (reinforce CRBs) - the experiential aspect of FAP — not just Rule 5 (promote generalization) as has been 
emphasized in this paper. For example, when a client engages in a CRB2, the therapist, in addition to providing a reinforcing response, may help the client engage in self-talk that may function as reinforcement for the CRB2 as well. It is not difficult to imagine that such self-talk may provide reinforcing stimulus functions for previous client behavior, but it is beyond the scope of the current paper to expand on this idea as it suggests new approaches to FAP. In contrast, this paper focused primarily on clarifying existing FAP interventions. In addition, provision of homework assignments, which functionally may be seen as verbal rules that specify future behavior and expected consequences, especially those derived from Rule 5 discussions as discussed above, may further evoke improvement behavior (parallel to CRB2s) in the client's daily life. It is hoped that the paper provides theoretical clarity, clinical implications, and a useful framework for research and the collection of empirical data on these and related issues.

\section{$\underline{\text { References }}$}

Abreu, P. A., Hübner, M. M. C., \& Lucchese, F. (2012). The Role of Shaping the Client's Interpretations in Functional Analytic Psychotherapy, 28, 151-157.

Abreu-Rodrigues, J., Sanabio-Heck, E. T. (2004). Instruções e auto-instruções: contribuições da pesquisa básica. In: C. N. de Abreu; H. J. Guilhardi. (Orgs.). Terapia comportamental e cognitivo-comportamental. (Vol. 1; 1a ed - pp. 152-168). São Paulo: Roca.

Aron, A., Melinat, E., Aron, E. N., Vallone, R. D., \& Bator, R. J. (1997). The experimental generation of interpersonal closeness: A procedure and some preliminary findings. Personality and Social Psychology Bulletin, 23(4), 363-377.

Bicard, D. F., \& Neef, N. A. (2002). Effects of Strategic versus tactical instructions on adaptation to changing contingencies in children with ADHD. Journal of Applied Behavior Analysis, 35, 375-389.

Busch, A. M., Callaghan, G. M., Kanter, J. W., Baruch, D. E., \& Weeks, C. E. (2010). The Functional Analytic Psychotherapy Rating Scale: A replication and extension. Journal of Contemporary Psychotherapy, 40, 11-19. doi:10.1007/s10879-009-9122-8

Busch, A. M., Kanter, J. W., Callaghan, G. M., Baruch, D. E., Weeks, C. E., \& Berlin, K. S. (2009). A micro-process analysis of Functional Analytic Psychotherapy's mechanism of chance. Behavior Therapy, 40, 280-290.

Callaghan, G. M., \& Follete, W. C. (2008). FAPRS MANUAL: Manual for the Functional Analytic Psychotherapy Rating Scale. The Behavior Analyst Today. 9(1), 57-97. 
Callaghan, G. M., Naugle, A. E., \& Follette, W. C. (1996). Useful constructions of the client therapist relationship. Psychotherapy, 33(3), 381-390.

Callaghan, G. M., Summers, C. J., \& Weidman, M. (2003). The Treatment of Histrionic and Narcissistic Personality Disorder Behaviors: A Single-Subject Demonstration of Clinical Improvement Using Functional Analytic Psychotherapy. Journal of Contemporary Psychotherapy, 33(4), 321-339.

Callaghan, G. M. (2006a). Functional Assessment of Skills for Interpersonal Therapists: The FASIT System. The Behavior Analyst Today, 7(3), 399-433.

Callaghan, G. M. (2006b). The Functional Idiographic Assessment Template (FIAT) System. The Behavior Analyst Today, 7(3), 357-398.

Callaghan, G. M., Follette, W. C., Ruckstuhl Jr., L. E., \& Linnerooth, P. J. N. (2008). The Functional Analytic Psychotherapy Rating Scale (FAPRS): A Behavioral Psychotherapy Coding System. The Behavior Analyst Today, 9(1), 98-116.

Callaghan, G. M., \& Follete, W. C. (2008). FAPRS MANUAL: Manual for the Functional Analytic Psychotherapy Rating Scale. The Behavior Analyst Today, 9(1), 57-97.

Catania, A. C. (1998). Learning. Englewood Cliffs, NJ: Prentice-Hall.

Cautilli, J., Riley-Tillman, T. C., Axelrod, S., \& Hineline, P. (2005). The Role of Verbal Conditioning in Third Generation Behavior Therapy. The Behavior Analyst Today, 6(2), 137-146.

Fernández-Parra, A. \& Ferro, R. (2006). Psicoterapia analítico- funcional: una aproximación contextual funcional al tratamiento psicológico. EduPsykhé, 5(2), 203-229.

Follette, W. C., Naugle, A. E., \& Callaghan, G. M. (1996). A Radical Behavioral Understanding of the Therapeutic Relationship in Effecting Change. Behavior Therapy, 27, 623-641.

Ferro, R. (2008). Recent Studies in Functional Analytic Psychotherapy. International Journal of Behavioral Consultation and Therapy, 4(2), 239-249.

Hayes, S. C., Barnes-Holmes, D., \& Roche, B. (2001). Relational Frame Theory: A PostSkinnerian Account of Human Language and Cognition. NewYork: Kluwer Academic/PlenumPress.

Hayes, S. C., Kohlenberg, B. S. \& Melancon, S. M. (1989). Avoiding and altering rule-control as a strategy of clinical intervention. In: S. C. Hayes (Ed.), Rule-governed behavior: Cognition, contingencies, and instructional control (pp. 359-385). NY: Plenum Press. 
Hayes, S. C., Zettle, R. D., \& Rosenfarb, R. (1989). Rule-following. In: S. C. Hayes (Ed.), Rule-governed behavior: Cognition, contingencies, and instructional control (pp. 191-220). NY: Plenum Press.

Holmes, E. P., Dykstra, T. A., \& Williams, P. (2003). Functional analytic rehabilitation: A contextual behavioral approach to chronic distress. Behavior Analyst Today, 4, 34-46.

Hopko, D. R., \& Hopko, S. D. (1999). What can Functional Analytic Psychotherapy contribute to empirically-validated treatments. Clinical Psychology and Psychotherapy, 6, 349-356.

Johnston, J. M. (1979). On the relation between generalization and generality. Behavior Analyst, 2(2), 1-6.

Kanter, J. W., Callaghan, G. M., Landes, S. J., Busch, A. M., \& Brown, K. R. (2004). Behavior Analytic Conceptualization and Treatment of Depression: Traditional Models and Recent Advances. The Behavior Analyst Today, 5, 255-274.

Kanter, J. W., Landes, S. J., Busch, A. M., Rusch, L. C., Brown, K. R., Baruch, D. E., \& Holman, G. (2006). The effect of contingent reinforcement on target variables in outpatient psychotherapy for depression: A successful and unsuccessful case using functional analytic psychotherapy. Journal of Applied Behavior Analysis, 39, 463-467.

Kohlenberg, R. J., \& Tsai, M. (1991). Functional analytic psychotherapy: A guide for creating intense and curative therapeutic relationships. NY: Plenum.

Kohlenberg, R. J., Tsai, M., Parker, C. R., Bolling, M. Y. \& Kanter, J. W. (1999). Focusing on the Client-Therapist Interaction - Functional Analytic Psychotherapy: A Behavioral Approach. European Psychotherapy, 1(1), 15-25.

Landes, S. J., Kanter, J. W., Weeks, C. E., Busch, A. M. (2013). The impact of the active components of functional analytic psychotherapy on idiographic target behaviors. Journal of Contextual Behavioral Science, 2, 49-57.

Lizarazo, N. E., Muñoz-Martínez, A. M., Santos, M. M., \& Kanter, J. K. (in press). A Within Subjects Evaluation of the Effects of Functional Analytic Psychotherapy on In-Session and Out-of-Session Client Behavior. Psychological Record.

Maitland, D. W. \& Gaynor, S. T. (2012). Promoting Efficacy Research of Functional Analytic Psychotherapy. International Journal of Behavioral Consultation and Therapy, 7(2-3), 6371.

Mangabeira, V., Kanter, J. W., \& Del Prette, G. (2012). Functional Analytic Psychotherapy (FAP): A review of publications from 1990 to 2010. International Journal of Behavioral Consultation and Therapy, 7(2-3), 78-89. 
Meyer, S. B. (2000). Mudamos, em terapia verbal, o controle de estímulos?. Acta Comportamentalia, 8(2), 215-225.

Meyer, S. B. (2004). Processos comportamentais na psicoterapia. In: Ciência do Comportamento. Conhecer e Avançar (vol. 4, pp. 151-157). Santo André : ESETec.

Meyer, S. B. (2005). Regras e auto-regras no laboratório e na clínica. In: J. Abreu-Rodrigues \& M. R. Ribeiro (Orgs.), Análise do Comportamento: pesquisa, teoria e aplicação (pp. 211227). Porto Alegre: ARTMED Editora S.A.

Michael, J. (1982). Distinguishing between Discriminative and Motivational Functions of Stimuli. Journal of the Experimental Analysis of Behavior, 37(1), 149-155.

Michael, J. (1993). Establishing Operations. Journal of the Experimental Analysis of Behavior, 16(2), 191-206.

Oshiro, C. K. B.; Kanter, J. W., Meyer, S. B. (2012). A Single-Case Experimental Demonstration of Functional Analytic Psychotherapy with Two Clients with Severe Interpersonal Problems. International Journal of Behavioral Consultation and Therapy, 7(2-3), 111-116.

Pankey, J. (2012). Functional Analytic Psychotherapy (FAP) for Cluster B Personality Disorders: Creating Meaning, Mattering, and Skills. International Journal of Behavioral Consultation and Therapy, 7(2-3), 117-124.

Pryor, K. (1984). Don't Shoot the Dog. NY: Bantam.

Schlinger, H. \& Blakely, E. (1987) Function-Altering Effects of Contingency-Specifying Stimuli. The Behavior Analyst, 10(1), 41-45.

Skinner, B. F. (1953). Science and Human Behavior. NY: Macmillan Company.

Skinner, B. F. (1957). Verbal behavior. New Jersey: Prentice-Hall.

Skinner, B. F. (1966). An operant analysis of problem solving. In B Kleinmuntz (Ed.) Problem solving: Research, method and theory (pp. 133-171). New York: John Wiley \& Sons.

Skinner, B. F. (1969). Contingencies of Reinforcement: A theoretical analysis. New York: Appleton-Century-Crofts.

Skinner, B. F. (1974). About behaviorism. New York: Vintage books.

Törneke, N., Luciano, C., \& Valdivia Salas, S. (2008). Rule-governed behavior and psychological problems. International Journal of Psychology and Psychological Therapy, $8(2), 141-156$.

Tourinho, E. Z. (2006). O autoconhecimento na Psicologia Comportamental de B.F. Skinner. Santo André: ESETec Editores Associados. 
Tsai, M., Kohlenberg, R. J., Kanter, J. W., Holman, G. I., Loudon, M. P. (2012). Functional Analytic Psychotherapy. Cornwall: TJ International Ltd.

Tsai, M., Kohlenberg, R. J., Kanter, J. W., Kohlenberg, B., Follete, W. C., \& Callaghan, G. M. (2009). A guide to Functional Analytic Psychotherapy: Awareness, courage, love and behaviorism. New York: Spring.

Wechsler, A. M., \& Amaral, V. L. R. (2009). Correspondência verbal: uma revisão da literatura. Revista Brasileira de Terapia Comportamental e Cognitiva, 11(2), 189-208.

Weeks, C. E., Kanter, J. W., Bonow, J. D., Landes, S. J., Busch, A. M. (2012). Translating the Theoretical Into Practical: A Logical Framework of Functional Analytic Psychotherapy Interactions for Research, Training and Clinical Purposes. Behavior Modification, 36(1), 87-119. DOI: $10.1177 / 0145445511422830$

Wetterneck, C. T. \& Hart, J. M. (2012). Intimacy is a Transdiagnostic Problem for Cognitive Behavior Therapy: Functional Analytical Psychotherapy is a solution. International Journal of Behavioral Consultation and Therapy, 7(2-3), 167-176.

Xavier, R. N., Kanter, J. W., \& Meyer, S. B. (2012). Transitional Probability Analysis of Two Child Behavior Analytic Therapy Cases. International Journal of Behavioral Consultation and Therapy, 7(2-3), 182-188. 
A seguir será dada continuidade aos demais temas abordados na Introdução da presente Tese.

\section{Investigações a respeito dos mecanismos de ação da FAP}

Como mencionado anteriormente, pesquisas descritivas e experimentais vêm sendo realizadas na área da FAP a fim de se investigar seus principais mecanismos de ação. Apesar de promissoras, tais pesquisas precisam ainda de mais investimentos, a fim de aumentar especialmente os dados experimentais produzidos e dar maior sustentação às evidências de sua eficácia e mecanismos de ação (Ferro, 2008; Ferro \& Valero, 2015; Maitland \& Gaynor, 2012; Mangabeira, Kanter, \& Del Prette, 2012; Tsai et al., 2009; Weeks et al., 2012).

Há muito tempo, na área da análise do comportamento, vem sendo adotado o delineamento de sujeito único como importante e principal forma de pesquisa. Nessa forma de investigação, poucos sujeitos são investigados, mas seus comportamentos são analisados ao longo do tempo com a introdução e retirada de variáveis (Andery, 2010; Matos, 1990; Sampaio et al., 2008; Sidman, 1960). Tal forma de investigação vem sendo utilizada também em pesquisas clínicas e aplicadas e não apenas em um contexto protegido de laboratório, com maior controle de variáveis e tem auxiliado na obtenção de respostas sobre a eficácia de tratamentos, por exemplo. Tal forma de investigação vem sendo denominada como delineamento de caso-único e segue os mesmos preceitos dos delineamentos de sujeito-único (para discussões ou apresentações de delineamentos de caso único ver Hayes, 1981; Kazdin, 2002; Kratochwill et al., 2013; Smith, 2012). O delineamento de caso único pode, portanto, ser utilizado em um contexto clínico, permitindo a análise de como variáveis que são introduzidas ou retiradas do processo terapêutico podem afetar o comportamento do indivíduo que está sendo submetido ao delineamento. Ainda mais vantagens são obtidas quando é possível analisar detalhadamente o que acontece dentro da sessão experimental, com o auxílio de instrumentos de categorização, frente a introdução e retirada dessas variáveis e não apenas seus resultados gerais.

Um importante instrumento que possibilitou o aumento de pesquisas na área da FAP, foi a criação da Functional Analytic Psychotherapy Rating Scale (FAPRS), descrito em Callaghan e Follette (2008) e Callaghan, Follette, Ruckstuhl e Linnerooth (2008). Tal instrumento define categorias de comportamentos importantes em uma interação FAP para as falas de terapeuta e cliente a fim de se identificar como cada um se comportou em sessão e com qual sequência de interação. A criação de tal instrumento viabilizou diversas pesquisas de caso-único sobre os 
efeitos da aplicação da FAP, que passaram a identificar detalhadamente a interação entre terapeuta e cliente e permitiram a verificação dos mecanismos de ação relevantes na abordagem (Busch et al., 2009, 2010; Callaghan et al., 2003, 2008; Geremias, 2014; Lizarazo et al., no prelo; Mangabeira, 2014; Meurer, 2011; Oshiro et al., 2012; Popovitz, 2013; ver Del Prette, 2011 e Xavier, Kanter \& Meyer, 2012 para exemplos de uso do instrumento em terapia comportamental infantil).

Callaghan et al. (2003), por exemplo, investigaram os efeitos de um atendimento realizado a partir da abordagem da FAP, através da categorização com o sistema FAPRS de trechos de 15 minutos de algumas sessões terapêuticas. Entre outros detalhamentos, foi possível observar que o cliente passou a apresentar mais CCR2s e CCR3s e menos CCR1s ao longo do processo terapêutico. Além disso, através de uma análise sequencial a fim de identificar como o terapeuta respondeu aos CCRs, concluiu-se que o mesmo foi consistente ao modelo da FAP, apoiando que tal responder contingente é um importante mecanismo de ação da FAP.

Em Kanter et al. (2006), dois clientes foram atendidos, passando por um delineamento quase-experimental no qual foram realizadas sessões de Terapia Cognitiva-Comportamental (TCC) e, em seguida, adicionadas respostas condizentes com o modelo da FAP (Delineamento $\mathrm{A} / \mathrm{A}+\mathrm{B})$. Nesse trabalho foram analisadas as melhoras apresentadas pelos clientes fora do contexto terapêutico (mais detalhes dessa publicação adiante). Alguns anos depois dessa publicação, Busch et al. (2009) analisaram as sessões realizadas com um dos clientes, categorizando-as na íntegra com o sistema FAPRS e verificaram que o CCR1 mostrou-se variável tanto na fase de TCC, como na de FAP; enquanto o CCR2 aumentou grandemente em porcentagem de emissão na fase FAP em comparação com a anterior. Além disso, foram feitas análises sequenciais sobre o responder do terapeuta em relação aos CCRs da cliente e constatou-se que o terapeuta emitiu respostas de modelagem de CCRs apenas na fase FAP o que apoia a hipótese sobre o responder do terapeuta aos CCRs ser o principal mecanismo de mudança da FAP.

Por sua vez, Busch et al. (2010) analisaram os comportamentos do cliente e do terapeuta ao longo de sessões de uma terapia FAP e verificaram que o CCR2 aumentou, enquanto o CCR1 diminuiu em porcentagem de emissão ao longo das sessões, apesar de apresentarem variações em suas porcentagens. Além disso, como nos trabalhos anteriores, análises sequenciais sobre o responder do terapeuta aos CCRs indicaram que respostas contingentes e efetivas foram emitidas, apoiando mais uma vez as hipóteses sobre o responder contingente ser o principal mecanismo de ação da FAP. 
Tais trabalhos trouxeram grande avanço na área ao mostrarem ser possível a categorização de cada resposta emitida em sessão através do sistema FAPRS, identificando o responder contingente do terapeuta aos comportamentos do cliente. Apesar das evidências nos três trabalhos de que o responder contingente aos CCRs é um importante mecanismo de ação da FAP os autores reconhecem que mais pesquisas são necessárias, especialmente por estes serem trabalhos com delineamentos de caso único. Delineamentos como estes mostram fortes evidências sobre o que ocorreu com aquele cliente em questão, mas não levam a generalização dos dados para outros clientes e por isso, novas investigações são necessárias. Busch et al. (2009, 2010) sugerem ainda que outras variáveis pertencentes ao modelo da FAP sejam analisadas a fim de se investigar se há outros mecanismos de ação envolvidos em tal processo e sugere novos delineamentos experimentais, como linha de base múltipla através de sujeitos ou classes de respostas e delineamentos A-B, para melhor controle da apresentação de tais variáveis.

Em 2012, Oshiro et al. publicou uma pesquisa na qual foi realizado um controle experimental maior do que nos trabalhos descritivos de Callaghan et al. (2003) e Busch et al. (2010) e do que o delineamento quase-experimental de Kanter et al. (2006), ao utilizar um delineamento também de caso-único, mas acrescentando um delineamento de reversão. Assim como no delineamento realizado por Kanter et al. (2006), Oshiro et al. (2012) controlou o momento de inclusão da FAP na terapia, mas em seguida retirou-a para, por fim, acrescentá-la e retirá-la novamente, com dois participantes (delineamento A-B1-A1-B2-A2, sendo A terapia comportamental com foco em análise de contingências externas e B terapia realizada nos moldes da FAP).

Com o delineamento experimental utilizado por Oshiro et al. (2012), são respeitadas as mudanças pelas quais o participante passa, no decorrer do tempo, levando em consideração sua história individual, como nos demais delineamentos de caso-único. Porém, especialmente em um delineamento de reversão, comparações entre diferentes condições experimentais podem ser feitas, através da apresentação e retirada de uma ou mais variáveis independentes em condições diferentes do experimento. Tais apresentações de variáveis permitem comparações dos efeitos de cada uma delas (variável independente) sobre o comportamento analisado (variável dependente), sendo possível a reversão de uma condição a outra. A grande vantagem desse tipo de delineamento é que ele permite grande confiabilidade sobre o efeito da variável no comportamento observado, caso o comportamento apresente de fato modificação em função da retirada e apresentação da variável (Kazdin, 2002; Sampaio et al., 2008). Porém, a depender das variáveis manipuladas, a reversão pode não ser desejável na clínica. Se um tratamento leva 
à melhora, deve-se mantê-lo e não retirá-lo permitindo a ocorrência de uma piora (o que seria eticamente inaceitável), além da dificuldade prática de se interromper um tratamento que vem tendo sucesso (Kazdin, 2002). Por outro lado, se são testados diferentes tipos de intervenção já utilizados na área, de modo que a variável a ser introduzida e retirada não leva a prejuízos evidentes no tratamento, o delineamento de reversão pode ser uma ótima ferramenta para verificar a efetividade de cada tipo de intervenção e identificar formas mais eficientes de se fazer terapia. O efeito da intervenção é claro se com a apresentação da variável verifica-se melhora, com a retirada da variável observa-se reversão ou semelhança ao desempenho de linha de base, e com a reapresentação da variável, uma nova melhora (Kazdin, 2002).

Oshiro et al. (2012) entenderam que a psicoterapia realizada nos moldes da FAP poderia ser uma variável a ser introduzida e retirada do processo sem causar ônus ao tratamento e especialmente ao cliente e observaram exatamente o resultado descrito por Kazdin (2002) de variações nas variáveis dependentes (CCR1s e CCR2s, no caso) sistemáticas de acordo com a apresentação e retirada da variável independente (no caso a FAP). Ou seja, foi observado que para ambos os clientes, nas fases FAP houve a diminuição de comportamentos problema e aumento de comportamentos de melhora, sendo que o inverso ocorreu nas fases com análise de contingências externas. Ainda para um dos clientes, mesmo com o aumento de CCR 1 e a diminuição de CCR2 observada na Fase A1 (quando retirada pela primeira vez a FAP) a porcentagem de emissão dessas respostas não atingiu a mesma observada na Fase A; ou seja, mesmo que uma piora no responder da cliente tenha sido observada nas fases sem a conduta da FAP, ocorreu provavelmente algum efeito de aprendizagem já que a cliente não voltou a apresentar problemas com a mesma intensidade que apresentou antes da introdução da FAP.

Oshiro et al. (2012) descrevem ainda que com isso observa-se uma validade interna (replicação intra-sujeitos) e externa (replicação inter-sujeitos) para os dados apresentados, gerando a mais forte evidência da literatura até então de que o responder contingente do terapeuta pode efetivamente levar à modelagem de CCRs. Além disso, os dados claramente apresentados, com os dois clientes da pesquisa, indicam esse delineamento como sendo um bom meio de estudar as variáveis que influenciam um processo terapêutico.

De fato, outros três trabalhos seguiram o de Oshiro et al. (2012), realizando também delineamentos de reversão com introdução e retirada da FAP ou de uma de suas propriedades, em diferentes fases experimentais (Geremias, 2014; Mangabeira, 2014; Popovitz, 2013).

Popovitz (2013) realizou uma tentativa de investigação sobre o que ocorre em sessões FAP, porém focando-se no responder aos CCR1s. Na pesquisa da autora, duas díades de terapeuta e cliente submeteram-se a um delineamento experimental de caso-único com 
reversão, do tipo A-B-A-B, no qual A representa principalmente um responder do terapeuta a CCR2 e CCR3, condizente com o previsto pela FAP, enquanto CCR1 não seria consequenciado; e B representa o mesmo responder a CCR2 e CCR3 da fase anterior, mas com o acréscimo de respostas de bloqueios a CCR1 de fuga/esquiva. Os dados não são claros em relação às diferenças quanto a forma de responder a CCR1. Mas, após análises sobre a probabilidade de um evento $\mathrm{X}$ ser seguido por um evento $\mathrm{Y}$, observou-se mais uma vez que quando o CRB2 foi consequenciado adequadamente, sua probabilidade de emissão aumentou, ao menos para uma das clientes.

Geremias (2014) planejou também realizar, com uma cliente, um delineamento de reversão do tipo A-B-A-B, sendo A terapia comportamental com foco em análises externas e B terapia nos moldes da FAP. Porém, devido à desistência da cliente no meio do delineamento, acabou por coletar dados apenas de A-B-A, chegando a fazer portanto a retirada da variável independente (a terapia seguindo os moldes da FAP). A autora observou em seus dados a corroboração dos dados encontrados anteriormente por Oshiro et al. (2012). Mais especificamente, a autora observou um aumento de CCR2, correspondente ao responder da terapeuta na fase na qual foi introduzida a FAP. Além disso, observou-se menor mudança no CCR1 do que no CCR2 com a introdução da FAP, mas paralelo a isso o responder contingente a CCR1 também sofreu menos modificação na fase com o manejo da FAP. Ou seja, a terapeuta respondeu de forma semelhante aos CCR1 em todas as fases do experimento, observando-se poucas diferenças na porcentagem de emissão dessa resposta, enquanto que o responder ao CCR2 foi consideravelmente diferente na fase FAP, sendo provavelmente o grande responsável pelo seu aumento. Mais uma vez tais dados dão suporte à hipótese sobre o principal mecanismo da FAP ser o responder contingente aos CCRs.

Ainda, Mangabeira (2014) realizou, com dois clientes, também um delineamento de caso único, com delineamento de reversão. O autor estava interessado em estudar duas formas diferentes de evocar e responder aos CCRs dos clientes, uma que fosse mencionando aspectos diretamente da relação terapêutica (chamada pelo autor de FAP sinalizada) e outra que fosse sem mencionar tais aspectos diretamente (chamada de FAP não-sinalizada). Realizou portanto um delineamento de reversão, no qual foram alternadas fases com essas duas modalidades de FAP. Intercaladas com essas fases foram também realizadas fases nas quais a FAP não foi conduzida, focando-se em análise de contingências externas, como nos trabalhos de Geremias (2014) e Oshiro et al. (2012). Desse modo, o delineamento realizado pode ser descrito como A-B-A-C-A-B-A-C-A, sendo A foco em análises de contingências externas; B, FAP sinalizada e C, FAP não sinalizada. Para o segundo participante, B e C foram apresentados em ordem 
inversa. Não foram observadas diferenças em relação a emissões de CCRs entre os dois tipos de condução da FAP utilizados (FAP sinalizada e FAP não-sinalizada). Porém, observou-se diminuição na porcentagem de emissão dos CCR1s e aumento na de CCR2 nas fases FAP em relação às não FAP, relacionado com o aumento ou diminuição do responder contingente do terapeuta aos CCRs, o que corroborou mais uma vez os dados encontrados anteriormente na literatura, sobre esse ser o principal mecanismo de ação da FAP.

A partir desse conjunto de dados observa-se que avanços tem sido feitos quanto ao estudo da FAP e sua aplicação, em especial à modelagem de comportamento ocorrida em sessão, mas um outro fator importante da condução de um processo terapêutico necessita ainda de maior atenção, condizente com a coleta de dados externos à terapia.

\section{Evidências de melhoras externas}

Alguns dos trabalhos mencionados acima, fizeram tentativas de obter dados sobre a melhora do comportamento fora de sessão, mas nem todos obtiveram sucesso (mais detalhes adiante). Uma das formas de se obter tais registros é a partir do relato do cliente em sessão sobre seus comportamentos externos. Na literatura da FAP, os relatos ocorridos em sessão sobre o que ocorre fora da sessão vêm sendo nomeados de outside-of-session behaviors (comportamentos fora de sessão) e recebem a nomenclatura de Os, com a separação de O1 quando diz respeito a situações problemas e $\mathrm{O} 2$, quando diz respeito a melhoras (Tsai et al., 2009).

Assim, Callaghan et al. (2003) fizeram uma tentativa de registro de melhoras externas através dos $\mathrm{O} 1 \mathrm{~s}$ e $\mathrm{O} 2 \mathrm{~s}$ emitidos em sessão e observaram que O1s diminuíram da primeira para a última sessão analisada, enquanto O2s aumentaram. Embora tenham sido emitidos pelo cliente com uma baixa porcentagem nas sessões terapêuticas, esses dados são condizentes com os dados observados dentro de sessão pelos autores (quanto a CCR1 e CCR2) e com os resultados do Inventário de Depressão de Beck (BDI) aplicado ao início e final da pesquisa.

Busch et al. (2010), por sua vez, também tentaram analisar os Os emitidos em sessão como medida de melhora externa, mas consideraram que tal análise não obteve sucesso por sua emissão ter sido muito variável e pelas regras de categorização impostas que restringem os Os a poucos exemplos. Uma categorização realizada pelo sistema FAPRS prevê que seja aplicada uma categoria para cada fala emitida em sessão e a definição de cada categoria ocorre de acordo com sua função. Assim, falas que tenham conteúdo de Os podem, em muitos casos, ser consideradas como um CCR pela função que apresenta dentro da sessão. Como o foco da FAP 
é a modelagem de comportamentos dentro de sessão, tais respostas seriam categorizadas como CCRs, "encobrindo" algumas (ou muitas) vezes relatos sobre o que ocorre fora da sessão. Desse modo, Busch et al. (2010) reconhecem que possivelmente houve melhora no dia a dia do cliente, como evidenciado através da aplicação de um instrumento padronizado (BDI) no início e ao final da terapia, mas discutem que melhores formas de coleta de dados externos precisam ser criadas em pesquisas futuras.

O trabalho de Kanter et al. (2006), mencionado anteriormente, foi focado nessa questão. Esses autores, se preocuparam com a construção de um registro diário para os clientes, no qual eles deveriam anotar a frequência com que emitiam certas respostas em seu dia a dia. As respostas a serem registradas foram definidas em conjunto com os próprios clientes e com o apoio do Formulário de Avaliação Ideográfica Funcional (Functional Idiographic Assessment Template - FIAT, Callaghan, 2006), a fim de serem identificadas classes de respostas dos clientes a serem trabalhadas em terapia. Como mencionado anteriormente, o delineamento adotado no presente estudo foi quase-experimental, do tipo $\mathrm{A} / \mathrm{A}+\mathrm{B}$, no qual A foi composta por TCC e B pela introdução da FAP, sendo que o comportamento externo foi registrado durante as duas fases experimentais. Quando a FAP foi introduzida, um dos clientes investigados, Dan, começou a apresentar problemas em continuar registrando seus comportamentos e acabou por largar a terapia. Melissa, a outra cliente investigada, permaneceu registrando seus comportamentos durante toda a pesquisa e tal registro indicou melhoras nas respostas externas à terapia depois de introduzida a FAP. Além disso, foi constatado que houve um responder contingente do terapeuta durante a fase FAP. Esses dados, em conjunto com aqueles apresentados por Busch et al. (2009), que fez análises detalhadas sobre o que ocorreu nas sessões de Melissa, indicam que as melhoras ocorridas com essa cliente em sessão foram acompanhadas de melhoras externas. Kanter et al. (2006), no entanto, discutiram ter sido uma limitação o fato de que as medidas externas foram registradas pela própria cliente e não por medidas observadas por outros, o que teria enriquecido o dado.

Um outro trabalho, realizado por Landes et al. (2013) também apresentou a preocupação em registrar os dados externos através de um registro diário realizado pelos próprios clientes. Os autores realizaram um delineamento quase-experimental de caso-único, com delineamento do tipo $\mathrm{A} / \mathrm{A}+\mathrm{B}$, sendo que $\mathrm{A}$ previa a construção do vínculo terapêutico previsto na FAP; e B os aspectos mais ativos da FAP, como evocar diretamente os CCRs e responder contingentemente a eles, assim como trabalhar na generalização dos ganhos para o ambiente externo. Assim, dois diferentes aspectos da FAP foram separados a fim de se observar qual aspecto da abordagem é o responsável por mais melhoras terapêuticas. O mesmo procedimento 
para registro externo adotado por Kanter et al. (2006) foi adotado por esses autores, com quatro clientes submetidos ao delineamento. Um dos clientes abandonou a terapia pouco depois da mudança de fase, os demais apresentaram melhoras nos comportamentos externos registrados ao longo da Fase A+B, indicando que os componentes mais ativos da FAP mostraram-se mais importantes para as melhoras externas dos clientes do que o desenvolvimento da relação terapêutica. Esse estudo trouxe grandes contribuições na área, ao ser o primeiro a demonstrar a possibilidade de melhoras fora de sessão a partir de procedimentos da FAP executados em sessão com diversos clientes. No entanto, algumas limitações são levantadas pelos próprios autores como o fato de que não foram feitas análises sobre o comportamento dos terapeutas em sessão a fim de se confirmar os mecanismos da FAP em ação e mesmo a melhora intrassessão dos clientes. Outra limitação diz respeito ao fato de não ter sido feito um trabalho durante a Fase A de focar nas respostas extra sessão e em sua modificação, enquanto isso foi feito na Fase A+B. Ou seja, a melhora extra sessão observada quando foi introduzida a FAP pode ter ocorrido pelos mecanismos de ação dessa abordagem ou podem ter ocorrido pelo foco de discussão nos comportamentos extra sessão.

$\mathrm{Na}$ tentativa de prosseguir as pesquisas isolando melhor essas variáveis, Lizarazo, Muñoz-Martínez, Santos e Kanter (no prelo) realizaram também um delineamento quaseexperimental de caso-único do tipo $\mathrm{A} / \mathrm{A}+\mathrm{B}$, com três clientes, no qual mantiveram presente em todas as fases experimentais, discussões sobre o comportamento fora da sessão com foco em mudanças nos mesmos. Tais comportamentos a serem modificados foram elaborados em conjunto com o cliente e com o auxílio do instrumento FIAT (Callaghan, 2006). Além disso, permitiram o uso das Regras 1 e 2 da FAP (evocações de CCRs) desde o início, sendo portanto adicionado as Regras 3, 4 e 5 (com ênfase na 3) na Fase A+B. Ainda, para um dos clientes, depois da Fase A+B, a coleta de dados teve continuidade, mas retirando-se a Regra 2 e mantendo-se as demais. Com dois dos três clientes que participaram da pesquisa, foi possível observar melhoras tanto dentro como fora de sessão, na Fase A+B. Apesar de considerarem que os resultados encontrados podem ser fruto da junção das Regras 2 e 3, os autores também consideram que a aplicação da Regra 3 pode ser suficiente para produzir melhoras dentro e fora de sessão. Por fim, dados de follow-up indicam que o efeito do delineamento perdurou ao menos por algumas semanas, sobre os comportamentos fora de sessão. Vale ainda apontar que os comportamentos do terapeuta em sessão foram categorizados, indicando que foram feitas poucas emissões de Regra 5 e ainda assim, passaram a ocorrer melhoras fora de sessão, sugerindo que essa talvez não seja necessária para que as melhoras do cliente ocorram fora de sessão. 
Dentre os trabalhos citados aqui, o único que mencionou a relação da Regra 5 com as melhoras extra sessão foi o de Lizarazo et al. (no prelo). Nos trabalhos de Kanter et al. (2006) e Landes et al. (2013) foram observadas melhoras fora do contexto terapêutico, mas não foi controlado ou medida a frequência de emissões de Regra 5, não sendo possível portanto, verificar se a hipótese da literatura de que esta seria responsável pelas melhoras extra sessão é verdadeira. Lizarazo et al. (no prelo) aponta uma direção para essas investigações, mas muito poderia ser acrescentado com pesquisas que manipulassem diretamente emissões da Regra 5 verificando-se seus efeitos sobre as melhoras ou pioras dos clientes. Além disso, questões relacionadas ao papel do CCR3 no processo de melhora do cliente, como mencionadas anteriormente, ficam ainda sem respostas.

Vale ainda apontar que nesses três trabalhos citados que observaram resultados fora de sessão (Kanter et al., 2006; Landes et al., 2013 e Lizarazo et al., no prelo), tal registro foi feito pelo próprio cliente e discutido com o terapeuta em sessão. A cada semana terapeuta e ciente olhavam juntos o registro realizado pelo cliente, discutindo-o e corrigindo-o caso necessário. Apesar do comportamento de auto registro não ser simples de ser adquirido, os terapeutas podem ter influenciado o que foi registrado de forma a trazer questionamentos sobre os dados coletados.

Por outro lado, Busch et al. (2010) e Callaghan et al. (2003) fizeram tentativas de analisar os Os em sessão, como já mencionado, e consideraram que poucas respostas haviam sido obtidas, gerando pouca confiabilidade nos registros, ainda que trouxessem indícios de melhora, como no caso de Callaghan et al. (2003). Porém, é importante apontar que esses últimos observaram uma queda de $7 \%$ para $0 \%$ de $\mathrm{O} 1$ da primeira para a última sessão analisadas e um aumento de $0 \%$ para $13 \%$ de $\mathrm{O} 2$ no mesmo período. Kanter et al. (2006), por sua vez, registrou os dados fora de sessão a partir do registro de quantos dias da semana obteve ocorrências do comportamento alvo e não através da frequência real de ocorrência de tais respostas, ou seja, o número máximo que poderia ser registrado seria sete (ocorrências do comportamento em sete dias da semana). Landes et al. (2013) registrou a frequência de respostas por semana, mas o cliente que mais emitiu respostas em uma semana foi o Cliente 2, atingindo dez respostas em uma semana, seguido pelo Cliente 4, que atingiu sete respostas em uma semana; os demais emitiram quatro ou menos respostas por semana. O único dos trabalhos que apresentou um registro extra sessão com frequências mais altas foi o de Lizarazo et al. (no prelo), atingindo um máximo de 25, 70 e 20 respostas para cada um dos três clientes. Ou seja, com exceção desse último todos os demais trabalhos aqui citados que coletaram dados fora de sessão terapêutica, basearam-se em números baixos para discutir as melhoras ocorridas fora de sessão 
(seja através da análise de Os, seja através de registro externo); e possivelmente essa é uma característica comum a esse tipo de registro. Certamente a frequência de registro depende fortemente da natureza da resposta escolhida para ser registrada, mas parece que tem sido comum que respostas pouco frequentes sejam selecionadas nas pesquisas em FAP.

É importante apontar que para se ter maior clareza de que os procedimentos realizados em sessão, nos moldes da FAP têm efeito sobre os comportamentos fora de sessão, é necessário que as respostas escolhidas a serem observadas fora, sejam funcionalmente semelhantes às repostas escolhidas a serem evocadas e modeladas dentro de sessão. Assim, a escolha de tais respostas nem sempre é tarefa fácil, já que devem ser escolhidas em concordância com o cliente e serem passíveis de identificação por ele, se a opção for utilizar registro externo. Para muitos clientes, registrar seu comportamento fora de sessão, nem sempre é uma tarefa fácil e necessita de algum treino. Porém, em um contexto de pesquisa pode ser difícil realizar um treino suficiente para garantir a precisão do registro, que não influencie de alguma forma no delineamento experimental. A solução encontrada pelos autores acima foi a de acompanhar de perto o que estava sendo registrado, modelando esse comportamento durante o período de pesquisa. Pergunta-se aqui se existiriam outras formas de se aumentar a confiabilidade do registro, sem que o terapeuta influenciasse tanto no registro realizado pelo cliente.

Sendo assim, faz-se importante refletir sobre as diferentes formas de coleta de dados fora de sessão e quais poderiam beneficiar mais as evidências de melhoras dos clientes em terapias e pesquisas sobre a FAP.

\section{Medidas extra sessão}

O objetivo aqui ao levantar questões relacionadas com medidas externas, não é o de fazer um extenso levantamento da bibliografia sobre o tema. Muito já foi investigado sobre isso e sabe-se das dificuldades relacionadas à área. Conforme mencionado por Hatfield e Ogles (2004), não há uma forma universal de medida aceita pelos clínicos e, desse modo, faz-se interessante uma reflexão sobre as diversas formas de coleta de dados externos, suas características e os benefícios que poderiam trazer para as pesquisas em FAP.

Sendo assim, vale refletir sobre o trabalho publicado por Lambert e Hawkins (2004), a respeito de formas de coletas de dados sobre os resultados da terapia e acabam por discutir a dificuldade em escolher qual instrumento utilizar para essa coleta. Apesar dos autores levantarem essa questão no âmbito do consultório, sem fins de pesquisa, acredita-se aqui que muitos pesquisadores passam por semelhantes dificuldades de escolha pela grande variedade 
de instrumentos e suas características. Os autores apontam, então, que essa escolha deve ser pautada na confiabilidade e validade do instrumento, mas discutem ainda alguns âmbitos sobre os quais essa decisão deve ser pautada: conteúdo, fonte, método de coleta e orientação temporal do instrumento.

Em relação ao conteúdo, os autores apontam que instrumentos podem investigar sintomas de diversos transtornos psicológicos ou de apenas um transtorno. Podem apontar também para três características das pessoas: a cognição individual, emoções ou comportamentos ${ }^{5}$. Por fim, podem ainda dizer respeito a mudanças ocorridas internamente na pessoa, ou em domínios interpessoais ou sociais.

Nas pesquisas sobre FAP mencionadas que coletaram dados externos à sessão e não se utilizaram de análise de Os (Kanter et al., 2006; Landes et al., 2013 e Lizarazo et al., no prelo), o principal dado coletado diz respeito a registros individuais, não relacionados a nenhum diagnóstico mais geral, mas a padrões idiossincráticos de comportamentos considerados problemas ou, mais raramente, de melhora, com foco em respostas observáveis e de relacionamento interpessoal. Tais escolhas efetuadas são consistentes com a teoria estudada e com as mudanças realizadas em sessão, devido ao paralelo esperado entre contextos de dentro e de fora da sessão terapêutica.

Hatfiled e Ogles (2004) realizaram uma pesquisa a partir de uma amostra de psicólogos americanos e identificaram que dentre aqueles que coletam dados em sua prática clínica, a maioria utiliza tanto instrumentos padronizados (tais como BDI, Goal attainment scaling GAS, CBCL, entrevista clínica estruturada, SCL-90-R), como instrumentos individualizados/não padronizados. Pode-se questionar a razão disso, e supor que ambas as formas de medidas trazem vantagens em serem aplicadas e talvez até sejam complementares. Korotitsch e Nelson-Gray (1999) mencionam, por exemplo, o fato dos instrumentos padronizados serem mais facilmente e rapidamente aplicáveis do que medidas de autoobservação. É certo que, pela FAP ser uma abordagem que trata o ser humano de forma funcional e portanto idiossincrática, é importante serem analisadas evoluções pessoais, focadas no que está sendo trabalhado em sessão. Porém, um instrumento mais geral, validado, capaz de identificar mudanças mais amplas e que possa ser aplicado em diversas pesquisas, permitindo comparação entre estudos, pode ser de grande valia, ao complementar e apoiar os resultados idiossincráticos coletados por registros individuais.

\footnotetext{
${ }^{5}$ Entendendo-se aqui como uma visão mais geral do termo comportamento e não uma visão comportamental, que incluiria também emoções.
} 
De fato, muitos dos estudos aqui relatados se beneficiaram da aplicação de algum instrumento padronizado (escalas de depressão, sendo a BDI a mais frequente), como forma auxiliar na confirmação dos resultados coletados por instrumentos individuais ou análises realizadas em sessão (Busch et al., 2009, 2010; Callaghan et al., 2003; Landes et al., 2013; Meurer, 2011; Popovitz, 2013).

Em relação à fonte de coleta de dados, Lambert e Hawkins (2004) apontam que inequivocamente é muito melhor trabalhar com múltiplas fontes de dados a fim de medir o progresso da terapia. Certamente os dados obtidos pelos próprios clientes são importantes e muitas vezes mais significativos do que de outras fontes (Korotitsch \& Nelson-Gray, 1999; Lambert \& Hawkins, 2004), mas o complemento de registros de observadores externos (outras pessoas registrando o comportamento do cliente) pode ser valioso. Assim, apesar das dificuldades (inclusive de sigilo ético) em se ter observadores externos, dados dessas fontes legitimam os resultados observados e diminuem possíveis vieses que podem ocorrer com o registro obtido pelo cliente.

Starling (2010), por exemplo, menciona que apesar da importância do relato do próprio cliente, há problemas associados a relatos verbais (e auto-relatos) quando utilizados como variável dependente em uma pesquisa (Hersen \& Barlow, 1976, citado por Starling, 2010) e descreve tais problemas como sendo de reatividade da auto-observação (para mais detalhes, ver Bohm \& Gimenes, 2008, 2012; Foster, Laverty-Finch, Gizzo, \& Osantowski, 1999; Jackson, 1999; Korotitsch \& Nelson-Gray, 1999), efeitos da expectativa do terapeuta sobre o cliente, respostas de contra-controle e manipulações do comportamento verbal.

Além disso, Korotitsch e Nelson-Gray (1999) separam o comportamento de registro do comportamento externo feito pelo cliente em duas respostas: a necessária discriminação feita pelo cliente sobre seu comportamento; e o registro em si, de ocorrência da resposta, bem como algum registro adicional que, a depender do objetivo do registro, pode variar entre intensidade da resposta ou o estímulo antecedente, por exemplo. Desse modo, essas duas respostas são necessárias para que o registro seja efetuado com precisão e nem sempre os clientes a emitem a da forma ideal. Como apontam os autores, a maior preocupação em relação ao auto-registro utilizado com a função de coletar dados é em relação a sua precisão.

Tais problemas indicam que auto-registro de clientes, apesar de serem úteis e também bastante flexíveis como forma de coleta de dados (Korotitsch \& Nelson-Gray, 1999), não são sempre confiáveis e por isso pode ser útil complementá-los com relatos de outras fontes a fim de se confirmar sua precisão. Hatfiled e Ogles (2004) mostraram que, de fato, dentre os terapeutas que coletam dados sobre o progresso do cliente, é bastante frequente que os clientes 
respondam aos instrumentos de medida, assim como os próprios terapeutas. Outras pessoas também foram indicadas como respondendo alguma forma de registro, mas em menor quantidade, como pessoas importantes na vida do cliente, familiares, professores ou outros. É bem verdade que pode-se argumentar que observadores externos também necessitam das duas habilidades mencionadas por Korotitsch e Nelson-Gray (1999), de discriminação e de registro, para executar um trabalho de registro também confiável e que, desse modo, problemas podem ocorrer. No entanto, o que defende-se aqui é que, havendo a possibilidade de se ter também um observador externo, além do próprio cliente registrando seu comportamento, aumentam-se as chances de mais fidedignidade de informações sobre o que ocorreu de fato fora de sessão.

Nas pesquisas acima relatadas sobre as melhoras obtidas com o procedimento da FAP, os dados coletados fora de sessão, foram obtidos apenas pelos clientes e em nenhum caso por observadores externos ou mesmo o próprio terapeuta, apesar de suas impressões clínicas serem relatadas. Kanter et al. (2006) chegaram a mencionar a limitação em seu trabalho trazida pela ausência de um observador externo.

Lambert e Hawkins (2004) chamam ainda a atenção para o método utilizado na coleta de dados. Apontam que quanto mais específico for o método no qual se baseia um instrumento, menos a probabilidade de se detectar mudanças. No caso das pesquisas em FAP, isso pode ser considerado especialmente um problema. Por um lado, o objetivo das pesquisas é detectar melhoras nos CCRs que estão sendo trabalhados em sessão e em seus respectivos paralelos fora de sessão, de modo que os resultados esperados incidem sobre respostas específicas do repertório do cliente. Por outro lado, um problema surge por serem medidas muito específicas e por isso com poucas mudanças. Talvez isso explique a baixa quantidade de respostas externas observada nos dados das pesquisas acima relatadas, assim como a baixa quantidade de emissões de O1s e O2s, apontada nas pesquisas de Busch et al. (2010) e Callaghan et al. (2003); a única exceção, como dito anteriormente se deu na pesquisa de Lizarazo et al. (no prelo), que apesar de terem escolhido respostas específicas, estas foram observadas com grande frequência no cotidiano dos clientes. Possivelmente muito auxiliaria nessa questão se classes funcionais amplas fossem escolhidas para serem registradas pelos clientes e não apenas uma resposta definida em termos de topografia ou de algumas topografias limitadas. Mas a dificuldade encontra-se em auxiliar o cliente a compreender tal classe funcional já no início do processo terapêutico e experimental.

Por fim, Lambert e Hawkins (2004) chamam a atenção para a orientação temporal do instrumento que diz respeito às características medidas pelo instrumento em relação a traços ou estados. Ou seja, o instrumento está medindo algo que muda rapidamente com o tempo, ou 
características mais estáveis do indivíduo, que só serão modificadas com períodos maiores de tempo? Acredita-se que os pesquisadores da área têm feito boas escolhas quanto a isso ao medirem respostas rapidamente modificáveis, o que é comprovado por terem sido observadas mudanças em suas frequências ao longo do delineamento.

Além disso, há ainda a alternativa de analisar de forma qualitativa ou quantitativa o comportamento do cliente registrado através de vídeo ou áudio, tal como mencionado por Starling (2010). Busch et al. (2010) e Callaghan et al. (2003), utilizaram tais registros como uma tentativa de obter indícios de melhoras ou pioras externas, como já mencionado anteriormente. Dessa, forma, a partir do registro relato dos clientes sobre seus comportamentos externos foram analisados.

Certamente é possível questionar se o relato do cliente em sessão é realmente confiável, tanto por ser influenciado pelo terapeuta que poderia evocar mais falas de melhoras do que de pioras, por exemplo, quanto por desconfianças sobre a veracidade e precisão do que está sendo dito pelo próprio cliente. É bem verdade que muitos terapeutas se veem em situações nas quais questionam a veracidade do que o cliente está falando e, em muitos casos, certamente há razão para desconfianças. Um exemplo disso pode ser dado por falas incoerentes do cliente, nas quais menciona-se melhora, por exemplo, mas as situações contadas sobre o dia a dia não correspondem a tais melhoras. Ou quando pouco se obtém das situações do dia a dia do cliente, sendo os relatos bastante superficiais, por exemplo. Porém, quando essas razões para desconfianças não estão presentes, não há uma razão à priori para se desconfiar da não veracidade do que está sendo dito; ao menos não maior do que possíveis razões para desconfiar que o registro não está sendo preciso.

Sendo assim, acredita-se que nenhuma das formas de coleta de dados externos aqui mencionadas pode ser considerada ideal ou mesmo imparcial e por isso, acredita-se que múltiplas formas de coletas, utilizadas e analisadas em conjunto pode beneficiar mais as evidências externas dadas pelas pesquisas em FAP.

Nas palavras de Starling (2010)

Evidentemente, medir um fenômeno não é esgotar as possibilidades de conhecê-lo nos números que se obtém; medir um fenômeno é uma forma de obter um tipo de conhecimento sobre ele, que permite alguns tipos de ação que não são possíveis sem medidas. (p. 10)

Assim, medir um fenômeno de diversas formas, permite que se obtenha alguns tipos de conhecimento sobre ele, que podem ser complementares e tornando-os mais forte e confiáveis. 


\section{Justificativa e Objetivos}

Pretende-se dar continuidade às pesquisas que investigam os principais mecanismos de ação da FAP separando agora a parte experiencial da analítica e investigando seus efeitos dentro e fora de sessão. Muitas questões foram anteriormente levantadas sobre o papel das análises no processo da FAP e especialmente sobre os tipos de análise que poderiam ser mais efetivos para produzir melhoras. Não pretende-se aqui esgotar o assunto investigando-se todos esses aspectos das regras previamente levantados; pois afinal, para isso teriam que ser investigadas muitas variáveis ao mesmo tempo, o que inviabilizaria o controle experimental necessário para que conclusões sejam tiradas.

Duas perguntas então serviram como guia para a presente pesquisa:

1) Seria a parte analítica da FAP necessária para as mudanças previstas pela teoria tanto dentro como fora de sessão? 2) Não sendo necessária, a combinação entre as partes experiencial e analítica da FAP poderia auxiliar, potencializando talvez, as melhoras ocorridas com os clientes dentro e fora de sessão?

Para isso, então, foi realizado um processo terapêutico, seguindo o mais fielmente possível os principais pontos descritos na Interação Lógica da FAP. Dentro desse processo terapêutico, teve-se como objetivo investigar os efeitos diferenciais de análises realizadas pela terapeuta, através de Regra 5 (variável independente - VI), sobre 1) os comportamentos de análise dos clientes, assim como 2) seus comportamentos de melhora e piora dentro e 3) fora de sessão (variáveis dependentes - VD).

Para responder às questões o delineamento experimental de caso único, com reversões, do tipo A-B-BC-B-BC foi o selecionado. Neste, A é referente ao uso de terapia comportamental, sem foco na FAP; B, referente a introdução das Regras 1 a 4 da FAP; e C, manutenção das variáveis inseridas na fase anterior, com o acréscimo da Regra 5 da FAP. Análises incidiriam sobre o responder dos clientes em sessão, referentes a seus CCRs, bem como ao relato de comportamentos externos à terapia. Além disso, dados coletados fora de sessão, com instrumentos de coleta variados, serviriam para se obter várias medidas de melhora ou piora externa, visando uma maior fidedignidade ao dado.

A seguir serão descritos passo a passo os procedimentos utilizados para tal investigação. 


\section{MÉTODO}

\section{Participantes}

\section{1) Clientes}

Participaram da pesquisa duas clientes adultas (Dora e Sol, nomes fictícios) ${ }^{6}$, que procuraram por atendimento psicológico na clínica-escola do Instituto de Psicologia da Universidade de São Paulo. Para a seleção dessas clientes foram seguidos os seguintes critérios:

- Estivessem com idade entre 25 e 45 anos na ocasião do início da pesquisa;

- Estivessem cursando o Ensino Superior ou o já tivessem concluído, para que fosse maior a probabilidade de serem pessoas com um bom desenvolvimento de repertório verbal e maior probabilidade de aprenderem a emitir análises funcionais durante o período da presente pesquisa. Donadone (2009) observou uma correlação positiva estatisticamente significativa entre a escolaridade do cliente e um maior número de emissão de auto orientação, repertório relacionado com o estudado no presente trabalho;

- Apresentassem problemas de relacionamento interpessoal, pois esses são necessários para que se justifique o uso da FAP;

- Não apresentassem previamente diagnóstico de transtornos psiquiátricos de personalidade detectados durante a triagem.

\section{2) Observador externo}

Ao início do atendimento, juntamente com as informações sobre a presente pesquisa, as clientes foram informadas de que deveriam escolher um observador externo, adulto, com quem tivessem um relacionamento próximo e que fosse de sua confiança. O observador deveria registrar alguns dos comportamentos da cliente em momentos específicos da pesquisa. Foi esclarecido que os comportamentos selecionados para serem registrados seriam discutidos com a cliente previamente.

Ambas as clientes mostraram-se solícitas a isso, mas apenas a cliente Dora conseguiu selecionar alguém para cumprir essa função. Sol indicou três pessoas que julgou possível, mas que foram consideradas pela terapeuta como pessoas que abusavam dela em sua vida diária, de modo que não seriam indicadas a exercerem esse papel. Considerou-se que o registro dessas pessoas poderia prejudicar ainda mais esses relacionamentos e ser contra terapêutico.

\footnotetext{
${ }^{6}$ Uma terceira cliente foi atendida como precaução para o caso de desistência das demais, mas como não foi necessário a inclusão dela na pesquisa, seus dados não foram analisados.
} 
Durante toda a pesquisa, o registro do observador externo de Dora, bem como seu relacionamento com a cliente foram monitorados a fim de se verificar se estava sendo contraproducente para a melhora da cliente, o que não foi o caso.

\section{3) Pesquisadora-terapeuta}

A terapeuta é formada em psicologia pela Universidade de São Paulo, há dez anos na ocasião do início da coleta de dados, atuando desde então como terapeuta analíticocomportamental, utilizando FAP.

Antes de iniciado o período de coleta de dados, a terapeuta passou por um estágio sanduíche de dois meses na Universidade de Wisconsin-Milwaukee, sob supervisão do Prof. Dr. Jonathan Kanter, atualmente seu co-orientador. Durante esse estágio participou de atividades de ensino e pesquisa sobre FAP. Desde então tem aprimorado seu conhecimento teórico e prático sobre a abordagem. Para isso participou de workshops sobre FAP ministrados pelo co-orientador (agosto/2011, São Paulo e março/2012, Chicago), do treinamento online How to Give and Get Feedback to Shape CRB ministrado por Kelly Koerner e Gareth Holman (junho-julho/2012) e de Online Training sobre FAP liderado por Gareth Holman e Catherine McClafferty (novembro-dezembro/2012).

Ainda durante o estágio sanduíche passou a se aprimorar em atividades de elaboração e liderança de workshops para ensino de habilidades terapêuticas importantes na FAP. Coliderou um workshop com Jonathan Kanter (abril/2012, Kalamazoo), auxiliou na liderança de outros dois liderados por Mavis Tsai e Robert Kohlenberg (junho/2012, junho/2013) e de um terceiro, liderado por eles e por Jonathan Kanter (junho/2014, Seattle), além disso co-liderou um Online Training com Gareth Holman (abril-maio/2013). Tem realizado tais workshops no Brasil, liderando o primeiro workshop sobre FAP dado em português (outubro/2012, São Paulo), além de outros realizados no país desde então (outubro/2013, São Paulo; novembro/2013, Cuiabá; março/2014, Curitiba; abril/2014, Londrina; maio/2014, Curitiba).

Durante a coleta de dados com as clientes, a terapeuta foi supervisionada por seus coorientadores, com o objetivo de dar um bom andamento aos casos e dar continuidade no aprimoramento da sua forma de condução de terapia FAP. 


\section{4) Transcritores}

Três estudantes do último ano da graduação de Psicologia transcreveram as sessões atendidas pela terapeuta-pesquisadora, facilitando a posterior categorização, que será detalhada adiante.

\section{5) Aferidores de concordância}

Quatro psicólogas, terapeutas analítico-comportamentais foram as aferidoras de concordância. Três delas tinham experiência clínica de, no mínimo, três anos e conhecimento teórico e prático em FAP, sendo duas delas pesquisadoras experientes (Aferidoras 1 e 2). A quarta aferidora (Aferidora 4) era recém-formada, na época de exercer essa atividade, mas com experiência clínica durante a graduação, também utilizando FAP. Apesar da experiência clínica dessa última ter sido bem menor do que das demais, considerou-se interessante tê-la como aferidora pois ela foi um dos transcritores acima mencionados. Desse modo, tinha um conhecimento bastante aprofundado do caso.

A experiência como terapeuta utilizando FAP foi considerada importante para que as mesmas fossem capazes de reconhecer a ocorrência de CCRs e regras do terapeuta ocorrendo durante as sessões. As aferidoras leram uma conceituação do caso escrita pela pesquisadoraterapeuta a fim de ter o conhecimento necessário para analisar funcionalmente as sessões terapêuticas e passaram por um treino de identificação de CCRs e regras da FAP baseado na conceituação do caso, juntamente com a terapeuta. Mais detalhes sobre o procedimento realizado para se verificar a concordância serão apresentados adiante.

\section{Ambiente e equipamentos}

As sessões terapêuticas ocorreram, em geral, uma vez por semana com cada cliente e duração de cerca de 60 minutos. Foram realizadas em uma sala do Laboratório de Terapia Comportamental, localizado na clínica-escola do Instituto de Psicologia da Universidade de São Paulo. A sala de atendimento é composta por três cadeiras e uma mesa, relógio e possui um espelho unidirecional. Foi utilizado um gravador de áudio portátil da Samsung, posicionado em cima da mesa para o registro das sessões de Dora. Essa cliente não autorizou a filmagem das sessões, apenas a gravação em áudio. No caso de Sol, foi utilizada uma filmadora SONY acoplada no teto, além do mesmo gravador da Samsung posicionado em cima da mesa.

Para a análise de sessões as mesmas foram incialmente transcritas e posteriormente categorizadas utilizando-se um microcomputador Inspiron One 2330 da Dell, com processador 
Intel Inside, CORE i5, com Sistema Operacional Windows 8 Pro 64bit e Software Microsoft Office Home and Student 2013.

\section{Instrumentos}

\section{1) Instrumentos para coleta de dados}

Conforme discutido previamente, tentou-se, na presente pesquisa reunir a aplicação de diversas formas de coleta de dados externos à sessão, a fim de se aumentar as chances de se obter dados que fossem representativos e confiáveis. Desse modo, foi aplicado 1) um instrumento de auto registro baseado em respostas específicas de cada cliente, 2) um instrumento de auto registro padronizado, 3) um observador externo (apenas para a cliente Dora), 4) registro das sessões em áudio e/ou vídeo para análise de Os. Cada uma dessas formas de coleta de dados será descrita a seguir.

\section{1) Folhas de Registro do Comportamento do Cliente}

Relacionados aos CCRs definidos para cada cliente, foi desenvolvida uma folha de registro com os comportamentos problema e de melhora emitidos pelas mesmas fora de sessão, à semelhança do que foi realizado em Kanter et al. (2006), Landes et al. (2013) e Lizarazo et al. (no prelo). Na presente pesquisa, duas versões do instrumento foram criadas para a cliente Dora (uma a ser preenchida por ela e outra a ser preenchida pelo observador externo), enquanto apenas uma foi criada para a cliente Sol (detalhes sobre a conceituação de caso e sobre as respostas registradas serão apresentadas adiante.

Dora e Sol registraram a frequência diária de seus comportamentos, enquanto a folha preenchida pelo observador externo de Dora apresentava as respostas a serem registradas com uma escala de cinco pontos que correspondia às impressões que o observador teve nos últimos dois meses em relação a frequência de emissão de cada resposta, variando de raramente até com frequência. As folhas de registro podem ser observadas nos Apêndices A, B e C.

Tais registros permitiram uma quantificação das oportunidades de emissão de tais respostas e de suas emissões no dia a dia das clientes. Para a cliente Dora, as folhas de registro foram entregues semanalmente e ela as devolvia preenchidas na semana seguinte. O observador externo de Dora recebeu as folhas, via cliente e as enviou diretamente para a terapeuta via correio, com a recomendação de o fazer o mais rapidamente possível. A cliente Sol preferiu enviou a folha de registro preenchida para a terapeuta semanalmente via correio eletrônico. 
Apesar das clientes entregarem a folha semanalmente para a terapeuta, ela deveria ser preenchida diariamente, a partir dos acontecimentos daquele dia.

Diferentemente do que foi realizado em Kanter et al. (2006), Landes et al. (2013) e Lizarazo et al. (no prelo), a pesquisadora-terapeuta não entrou em contato com nenhum dos registros ao longo do delineamento e não interferiu neles para que estes não influenciassem na forma de trabalho em sessão (evitando que a VD influenciasse a VI, gerando um viés nos dados). A orientadora da pesquisa olhou os registros semanalmente para verificar se as clientes estavam de fato registrando as respostas, mas sem contabilizá-los. Esse procedimento foi explicado para as clientes.

\section{2) Escala de Avaliação dos Sintomas (EAS-40, Laloni, 2001)}

A EAS-40 foi um instrumento desenvolvido por Laloni (2001) a partir de um estudo de validação para a população brasileira do instrumento SCL-90-R (Symptom Checklist-90Revised) (Derogatis, 1994, apud Laloni, 2001). A SCL-90-R é um inventário de auto avaliação a partir de uma escala Likert de cinco pontos, que avalia problemas psicológicos e sintomas psicopatológicos e tem sido utilizada também como medida externa de melhora na avaliação de eficácia de drogas. Ao ser estudado para a população brasileira sofreu algumas adaptações, sendo reduzida para uma escala de 40 itens avaliados com uma escala Likert de três pontos e denominada como EAS-40. Os 40 itens mencionados são divididos em quatro dimensões, que compõem uma média geral do cliente, chamada de Índice Geral Global. As quatro dimensões são: Psicoticismo - avaliação de um contínuo entre psicose e depressão com sintomas de hostilidade e ideias paranóides; Obsessividade Compulsividade - repetição de pensamentos e ações, além de desconforto nas relações interpessoais; Somatização - sintomas de transtornos somáticos ou somatoformes; Ansiedade - avaliação tanto de ansiedade generalizada como ansiedade fóbica (Laloni, 2001). Desse modo, a EAS-40 é capaz de mostrar diferenças na população. A escala já foi validada para a população brasileira, tendo sido aplicada em diversos estudos, apresentando atualmente indicadores de parâmetros de normatização (Yoshida \& Silva, 2007).

Assim, a EAS-40 foi utilizada na presente pesquisa como instrumento auxiliar de medida do comportamento externo das clientes ao longo do processo psicoterápico. Para isso, foi aplicada a cada mudança de fase experimental (a serem descritas adiante) e também em momentos intermediários de cada fase, a fim de diminuir a possibilidade de identificação de mudanças de fase pelas clientes. 


\section{2) Instrumento para análise de dados}

3.2.1) Sistema de categorização da Psicoterapia Analítico Funcional (Functional Analytic Psychotherapy Rating Scale - FAPRS, Callaghan \& Follette, 2008) adaptado:

O FAPRS foi desenvolvido com o objetivo de categorizar funcionalmente $o$ comportamento de clientes e terapeutas durante a sessão terapêutica, baseado nos comportamentos considerados essenciais na FAP e outros importantes no processo terapêutico. Com esse instrumento é possível fazer o registro dos comportamentos de forma sequencial, identificando não só a frequência, como também as consequências dadas pelo terapeuta a cada CCR do cliente (Callaghan et al., 2003, 2008; Callaghan \& Follette, 2008; Weeks et al., 2012). Para análise do comportamento dos clientes foram criados códigos referentes aos CCRs e aos relatos de comportamentos problema $(\mathrm{O} 1)$ e de melhora $(\mathrm{O} 2)$ que ocorrem fora da sessão terapêutica. Os códigos relacionados ao terapeuta fazem referência a comportamentos considerados importantes para a condução da FAP, tais como focar na relação terapêutica e a forma de evocar e responder aos CCRs e aos Os. Sendo assim, CCRs e Os de cada cliente, bem como as formas de evocar e responder ao CCRs por parte do terapeuta são definidos a partir da conceituação do caso realizada.

Nas Tabelas 1 e 2, é apresentada uma descrição sucinta de cada um dos códigos utilizados na presente pesquisa, adaptados de Callaghan e Follette (2008, mais detalhes podem ser vistos na publicação original da escala). As adaptações realizadas foram desenvolvidas em conjunto com o Prof. Kanter e dizem respeito principalmente à adição das Regras 1, 4 e 5, sendo essa última separada entre análises funcionais sobre o comportamento do cliente, de pedidos de tarefas de casa e retirada de categorias nomeadas como missings no manual (referentes a deixar de responder apropriadamente a CCR1 e CCR2). Quanto às categorias do cliente, houve a inclusão da categoria O3 (análises sobre comportamento fora de sessão) separando-a da categoria de CCR3. 
Tabela 1: Códigos utilizados no FAPRS para categorização do comportamento do terapeuta (Callaghan \& Follette, 2008), adaptados.

\begin{tabular}{|c|c|c|}
\hline $\begin{array}{l}\text { Categoria } \\
\text { abreviada }\end{array}$ & Nome completo da categoria & Breve descrição \\
\hline Regra $1 *$ & $\begin{array}{l}\text { Terapeuta avalia CCR1 ou } \\
\text { CCR2 e/ou faz paralelo }\end{array}$ & $\begin{array}{l}\text { Terapeuta avalia CCR do cliente em sessão, } \\
\text { podendo fazer um paralelo de fora para dentro } \\
\text { da sessão. }\end{array}$ \\
\hline Regra 2* & $\begin{array}{l}\text { Terapeuta evoca CCR1 ou } \\
\text { CCR2 }\end{array}$ & $\begin{array}{l}\text { Terapeuta evoca, pede ou diretamente } \\
\text { (verbalmente) solicita um CCR. Esse código } \\
\text { NÃO depende do comportamento do cliente } \\
\text { que o segue. }\end{array}$ \\
\hline TRB1* & $\begin{array}{l}\text { Terapeuta responde } \\
\text { efetivamente ao CCR1 }\end{array}$ & $\begin{array}{l}\text { A resposta do terapeuta para comportamento } \\
\text { do cliente em sessão, incluindo a descrição de } \\
\text { sentimentos do terapeuta em resposta ao } \\
\text { cliente. }\end{array}$ \\
\hline TRB2* & $\begin{array}{l}\text { Terapeuta responde } \\
\text { efetivamente ao CCR2 }\end{array}$ & $\begin{array}{l}\text { Terapeuta responde efetivamente a melhoras } \\
\text { do cliente em sessão, incluindo a descrição de } \\
\text { sentimentos do terapeuta em resposta ao } \\
\text { cliente. }\end{array}$ \\
\hline $\mathrm{INF}^{*}$ & $\begin{array}{l}\text { Resposta ineficaz a CCR1 ou } \\
\text { CCR2 }\end{array}$ & $\begin{array}{l}\text { Terapeuta responde ao CCR de forma } \\
\text { grosseiramente ineficaz tanto por reforçar } \\
\text { CCR1 como por punir CCR2. }\end{array}$ \\
\hline Regra 4* & $\begin{array}{l}\text { Terapeuta verifica o efeito de } \\
\text { seu responder }\end{array}$ & $\begin{array}{l}\text { Terapeuta refere-se especificamente ou } \\
\text { pergunta sobre o efeito de sua resposta sobre } \\
\text { o comportamento do cliente. }\end{array}$ \\
\hline Regra 5-H & Terapeuta atribui tarefa de casa & $\begin{array}{l}\text { Terapeuta especificamente atribui tarefa de } \\
\text { casa baseado na interação em sessão. }\end{array}$ \\
\hline Regra 5* & $\begin{array}{c}\text { Terapeuta discute } \\
\text { (analisa/estabelece relações), } \\
\text { evoca ou modela } \\
\text { análises/estabelecimento de } \\
\text { relações }\end{array}$ & $\begin{array}{l}\text { Terapeuta busca por generalização do } \\
\text { comportamento do cliente, discutindo (dando } \\
\text { exemplo), evocando ou consequenciando } \\
\text { CCR3, O3 ou paralelos entre os dois. }\end{array}$ \\
\hline Regra 5-INF & $\begin{array}{l}\text { Resposta inefetiva a CCR3 ou } \\
\text { O3 }\end{array}$ & $\begin{array}{l}\text { Terapeuta responde ao CCR3 ou O3 de forma } \\
\text { grosseiramente inefetiva. }\end{array}$ \\
\hline TTR* & $\begin{array}{c}\text { Terapeuta foca na relação } \\
\text { terapêutica }\end{array}$ & $\begin{array}{l}\text { Terapeuta foca na relação terapêutica. } \\
\text { Não pode ser categorizado de outra forma. }\end{array}$ \\
\hline TPR* & $\begin{array}{l}\text { Terapeuta trabalha no avanço } \\
\text { positivo da sessão }\end{array}$ & $\begin{array}{l}\text { Terapeuta engaja em comportamentos } \\
\text { geralmente efetivos ou de facilitação, } \\
\text { incluindo discussões sobre tarefa de casa ou } \\
\text { programação quando isso não inclui um foco } \\
\text { especificamente em CCR ou Os. } \\
\text { Não pode ser categorizado de outra forma. }\end{array}$ \\
\hline TO1* & $\begin{array}{l}\text { Terapeuta fala sobre } \\
\text { comportamento externo } 1(\mathrm{O} 1)\end{array}$ & $\begin{array}{l}\text { Terapeuta descreve ou comenta } \\
\text { comportamento problema específico do } \\
\text { cliente que ocorre fora da sessão. }\end{array}$ \\
\hline $\mathrm{TO} 2 *$ & $\begin{array}{c}\text { Terapeuta fala sobre } \\
\text { comportamento externo } 2(\mathrm{O} 2)\end{array}$ & $\begin{array}{l}\text { Terapeuta descreve ou comenta } \\
\text { comportamento de melhora específico do } \\
\text { cliente que ocorre fora da sessão. }\end{array}$ \\
\hline
\end{tabular}


Tabela 2: Códigos utilizados no FAPRS para categorização do comportamento do cliente (Callaghan \& Follette, 2008), adaptados.

\begin{tabular}{|c|c|c|}
\hline $\begin{array}{l}\text { Categoria } \\
\text { Abreviada }\end{array}$ & Nome completo da categoria & Breve descrição \\
\hline CCR $1 *$ & $\begin{array}{l}\text { Comportamento Clinicamente } \\
\text { Relevante } 1 \text { (problema em } \\
\text { sessão) }\end{array}$ & $\begin{array}{l}\text { Cliente engaja em comportamento problema } \\
\text { na sessão, no contexto da relação } \\
\text { terapêutica. }\end{array}$ \\
\hline $\mathrm{CCR} 2 *$ & $\begin{array}{l}\text { Comportamento Clinicamente } \\
\text { Relevante } 1 \text { (melhora em } \\
\text { sessão) }\end{array}$ & $\begin{array}{l}\text { Cliente engaja em comportamento de } \\
\text { melhora na sessão, no contexto da relação } \\
\text { terapêutica. }\end{array}$ \\
\hline CCR3* & $\begin{array}{c}\text { Comportamento Clinicamente } \\
\text { Relevante } 3 \text { (análise sobre os } \\
\text { CCRs) }\end{array}$ & $\begin{array}{l}\text { Cliente descreve relação funcional (pelo } \\
\text { menos dois dos três termos da tríplice } \\
\text { contingência) relacionado aos CCRs } \\
\text { trabalhados em sessão e/ou faz paralelo de } \\
\text { dentro para fora da sessão. }\end{array}$ \\
\hline Pos-REP & Cliente relata efeito positivo & $\begin{array}{l}\text { Cliente relata uma resposta positiva às } \\
\text { reações do terapeuta. Frequentemente, mas } \\
\text { não necessariamente ocorre depois de CCR } \\
\text { ou TRB. }\end{array}$ \\
\hline Neg-REP & Cliente relata efeito negativo & $\begin{array}{l}\text { Cliente relata uma resposta negativa às } \\
\text { reações do terapeuta. Frequentemente, mas } \\
\text { não necessariamente ocorre depois de CCR } \\
\text { ou TRB. }\end{array}$ \\
\hline $\mathrm{O} 1 *$ & Comportamento externo 1 & $\begin{array}{l}\text { Cliente descreve problema específico que } \\
\text { ocorre fora de sessão. }\end{array}$ \\
\hline $\mathrm{O} 2 *$ & Comportamento externo 2 & $\begin{array}{l}\text { Cliente descreve melhora específica que } \\
\text { ocorre fora de sessão. }\end{array}$ \\
\hline O3* & Comportamento externo 3 & $\begin{array}{l}\text { Cliente descreve relação funcional (pelo } \\
\text { menos dois dos três termos da tríplice } \\
\text { contingência), relacionada a seu } \\
\text { comportamento externo que tenha relação } \\
\text { com CCRs. }\end{array}$ \\
\hline $\mathrm{CTR}^{*}$ & $\begin{array}{c}\text { Cliente foca na relação } \\
\text { terapêutica }\end{array}$ & $\begin{array}{l}\text { Cliente foca na relação terapêutica } \\
\text { Comportamento não é categorizável como } \\
\text { CCR. }\end{array}$ \\
\hline \multirow[t]{2}{*}{ CPR* } & \multirow[t]{2}{*}{$\begin{array}{l}\text { Cliente trabalha no avanço } \\
\text { positivo da sessão }\end{array}$} & $\begin{array}{l}\text { Cliente discute ou descreve problemas que } \\
\text { ocorrem em situações que não a relação } \\
\text { terapêutica, ou clarifica ou dá contexto } \\
\text { sobre os problemas. }\end{array}$ \\
\hline & & $\begin{array}{l}\text { Todos os comportamentos não } \\
\text { categorizáveis com outros códigos. }\end{array}$ \\
\hline
\end{tabular}

Na categorização com o FAPRS, como dito, cada fala do terapeuta e do cliente recebe um código, sendo possível observar a sequência de falas que ocorreu entre eles em sessão. 
Porém, algumas vezes, em uma mesma fala do terapeuta ou do cliente é possível observar respostas que poderiam receber diferentes códigos e nesses casos é utilizada uma hierarquia, pré-definida que orienta qual categoria deve ser utilizada. A hierarquia utilizada na presente pesquisa está sendo apresentada na Tabela 3. Esta segue o mesmo padrão das hierarquias criadas inicialmente para o sistema FAPRS, mas apresenta adaptações em função das mudanças na composição das categorias que estão sendo utilizadas.

Tabela 3: Hierarquia das categorias do FAPRS (Callaghan \& Follette, 2008), adaptada para a presente pesquisa.

\begin{tabular}{l}
\hline Para o terapeuta \\
\hline TRB $2>$ TRB $1>$ Regra $2>$ Regra $5>$ Regra $4>$ Regra $1>$ TTR $>$ TPR \\
\hline TRB $2>$ TRB $1>$ INF \\
\hline Regra $5>$ Regra 5 -H $>$ Regra 5-INF \\
\hline Regra $5>$ TO1, TO2 \\
\hline Para o cliente \\
\hline $\mathrm{CCR} 2>$ CCR $3>\mathrm{CCR} 1>\mathrm{O} 3>\mathrm{O} 2>\mathrm{O} 1>$ Pos-Rep $>$ Neg-Rep $>\mathrm{CTR}>$ \\
$\mathrm{CPR}$
\end{tabular}

Além das categorias descritas acima, para a presente pesquisa considerou-se interessante a criação de subcategorias referentes a um maior detalhamento daquelas que correspondem às análises emitidas por terapeuta e por cliente. Desse modo, as falas categorizadas como Regra 5, CCR3 e O3 foram subdivididas de acordo com as categorias apresentadas nas Tabelas 4 (para terapeuta) e 5 (para cliente). Assim as análises emitidas pelo terapeuta puderam ser divididas de acordo com seu conteúdo (comportamento problema x de melhora e sobre CCR $\mathrm{x}$ O3 x paralelos entre os dois) e de acordo com sua função (modelação/dar exemplo de análise x evocação do comportamento de análise do cliente x consequenciação da emissão de análise por parte do cliente). As análises emitidas pelas clientes foram divididas de acordo com seu conteúdo, da mesma fora que as do terapeuta (comportamento problema $\mathrm{x}$ de melhora e sobre CCR x O3 x paralelos entre os dois). Essas subdivisões foram consideradas como subcategorias e não categorias em si acrescentadas no sistema FAPRS pelas preocupações em relação a uma quantidade de categorias muito grandes e imprecisões em relação a elas, tais como levantadas previamente por Danna e Matos (1999), Meyer (2009) e Zamignani (2007). Assim, a principal categorização da presente pesquisa (e controle de variáveis independentes, conforme será 
descrito adiante) foi realizada apenas com as categorias acima e análises descritivas adicionais foram realizadas a partir das categorias descritas a seguir.

Tabela 4: Subcategorias criadas para Regra 5.

\begin{tabular}{|c|c|c|}
\hline Categoria subdividida & Nome completo da categoria & Breve descrição \\
\hline Regra 5-P* & Terapeuta faz paralelo & $\begin{array}{l}\text { Essa é uma subcategoria da } \\
\text { Regra } 5 \text { para ser codificada } \\
\text { apenas quando o terapeuta } \\
\text { busca por generalização ao } \\
\text { fazer especificamente um } \\
\text { paralelo de dentro para fora da } \\
\text { sessão. }\end{array}$ \\
\hline Regra 5-CCR3* & Terapeuta discute CCR3 & $\begin{array}{l}\text { Terapeuta discute } \\
\text { especificamente CCR3. }\end{array}$ \\
\hline Regra 5-O3* & Terapeuta discute $\mathrm{O} 3$ & $\begin{array}{l}\text { Terapeuta discute } \\
\text { especificamente } \mathrm{O} 3 \text {. }\end{array}$ \\
\hline Regra 5-EP & Terapeuta evoca paralelo & $\begin{array}{l}\text { Terapeuta especificamente } \\
\text { evoca paralelo para modelá-lo. }\end{array}$ \\
\hline Regra 5-RP & $\begin{array}{c}\text { Terapeuta responde } \\
\text { efetivamente ao paralelo }\end{array}$ & $\begin{array}{l}\text { Terapeuta responde } \\
\text { especificamente ao paralelo } \\
\text { para modelá-lo. }\end{array}$ \\
\hline Regra 5-E3 & Terapeuta evoca CCR3 & $\begin{array}{l}\text { Terapeuta especificamente } \\
\text { evoca CCR3 para modelá-lo. }\end{array}$ \\
\hline Regra 5-R3 & $\begin{array}{l}\text { Terapeuta responde } \\
\text { efetivamente ao CCR3 }\end{array}$ & $\begin{array}{l}\text { Terapeuta responde } \\
\text { especificamente ao CCR3 para } \\
\text { modelá-lo. }\end{array}$ \\
\hline Regra 5-EO3 & Terapeuta evoca $\mathrm{O} 3$ & $\begin{array}{l}\text { Terapeuta especificamente } \\
\text { evoca O3 para modelá-lo. }\end{array}$ \\
\hline Regra 5-RO3 & $\begin{array}{l}\text { Terapeuta responde } \\
\text { efetivamente ao } \mathrm{O} 3\end{array}$ & $\begin{array}{l}\text { Terapeuta responde } \\
\text { especificamente ao O3 para } \\
\text { modelá-lo. }\end{array}$ \\
\hline
\end{tabular}

*As categorias acima foram divididas a depender de seu conteúdo: sobre comportamentos problema (1) ou de melhora (2).

Da mesma forma como realizado para as categorias do FAPRS, as subcategorias acima apresentadas foram organizadas de acordo com uma hierarquia a fim de permitir que decisões padronizadas fossem tomadas quando mais de uma subcategoria era apresentada em uma mesma fala (Tabela 6). 
Tabela 5: Subcategorias criadas para CCR3 e O3.

\begin{tabular}{ccl}
\hline $\begin{array}{c}\text { Categoria } \\
\text { Abreviada }\end{array}$ & Nome completo da categoria & \multicolumn{1}{c}{ Breve descrição } \\
\hline CCR3* & $\begin{array}{c}\text { Comportamento Clinicamente } \\
\text { Relevante 3 (análise sobre os } \\
\text { CCRs) }\end{array}$ & $\begin{array}{l}\text { Cliente descreve relação funcional } \\
\text { (pelo menos resposta-consequência) } \\
\text { relacionado aos CCRs trabalhados em } \\
\text { sessão. }\end{array}$ \\
\hline CCR3-P* & $\begin{array}{c}\text { Comportamento Clinicamente } \\
\text { Relevante 3 - Paralelo }\end{array}$ & $\begin{array}{l}\text { Cliente faz paralelo de dentro para fora } \\
\text { da sessão. }\end{array}$ \\
\hline O3* & Comportamento externo 3 & $\begin{array}{l}\text { Cliente descreve relação funcional } \\
\text { (pelo menos resposta-consequência), } \\
\text { relacionada a seu comportamento } \\
\text { externo. }\end{array}$ \\
\hline
\end{tabular}

*As categorias acima foram divididas a depender de seu conteúdo: sobre comportamentos problema (1) ou de melhora (2).

Tabela 6: Hierarquia das subcategorias criadas para a presente pesquisa.

\begin{tabular}{l}
\hline Para o terapeuta \\
\hline Regra 5-P $>$ Regra 5-CCR3 $>$ Regra 5-O3 \\
\hline Regra 5-RP $>$ Regra 5-EP $>$ Regra 5-R3 $>$ Regra 5-E3 $>$ Regra 5-RO3 $>$ Regra \\
5-EO3 \\
\hline Para o cliente \\
\hline CCR3-P-2 $>$ CCR3-P-1 $>$ CCR3-2 $>$ CCR3-1 $>$ O3-2 $>$ O3-1 \\
\hline
\end{tabular}

\section{Procedimento}

A seguir serão descritas as etapas realizadas com as clientes para a condução da presente pesquisa. De forma geral três etapas foram realizadas: I) estabelecimento de vínculo terapêutico e conceituação de caso; II) o delineamento experimental propriamente dito; e III) encerramento da coleta de dados e seguimento da terapia.

\section{1) Etapa I - Estabelecimento de vínculo}

Foi dado início à terapia, a fim de estabelecer um bom relacionamento terapêutico com cada cliente, aumentando a adesão à terapia/pesquisa e diminuindo a chance de desistência. Nesse período inicial foi realizada por escrito a conceituação dos casos, levando à identificação de CCR1s, CCR2s e CCR3s, bem como seus paralelos para fora de sessão, específicos para cada cliente. Como apontam Follette e Bonow (2009) e Tsai et al. (2009), conceituações de caso são dinâmicas, o que poderia ter levado a mudanças nos comportamentos problema e de 
melhora identificados. Porém, por se tratar de uma pesquisa, é necessário haver permanência dos registros intra e extra sessão, ao longo das sessões para que seja possível a comparação dos dados coletados nas diferentes fases experimentais. Landes et al. (2013), por exemplo, descrevem como uma das limitações de sua pesquisa, o fato de que a FAP conduzida em sua pesquisa, talvez não corresponda fielmente a uma terapia FAP conduzida naturalmente no ambiente clínico. Isso porque foram escolhidos CCRs e comportamentos externos a serem registrados que fossem simples de serem registrados pelos clientes e que fossem de fácil mudança para que pudessem ser visualizados os efeitos da inserção de uma terapia FAP. Desse modo, não foi feita uma análise funcional, com definições de amplas classes de respostas, relacionadas a ampla conceituação de caso e nem tampouco uma introdução gradual da FAP. Como já mencionado anteriormente, Lambert e Hawkins (2004) apontam também a necessidade de escolha de respostas a serem registradas fora de sessão que sejam passíveis de rápidas mudanças.

Porém, o delineamento aqui proposto, como será melhor descrito posteriormente, envolve um longo período de coleta de dados, com vários meses de duração e por isso acredita-se ser importante que a pesquisa seja conduzida o mais fielmente possível ao que é realizado em um ambiente natural clínico. Sendo assim, em função do dinamismo encontrado na clínica, mas também das necessidades encontradas em uma pesquisa, os comportamentos problema e de melhora foram identificados como um contínuo, mas definindo-se previamente (até onde foi possível) quais as respostas seriam consideradas como problema ou como melhora. Além disso, tentativas de utilização da FAP foram realizadas nessa etapa, de forma não sistemática, a fim de se compreender características da interação terapêutica que permitam o uso apropriado da FAP com cada cliente. Nessa etapa foi realizada também a construção das Folhas de Registro de comportamentos das clientes a serem preenchidas por elas e pelo observador externo de Dora e iniciou-se um treino de preenchimento dessa folha, conforme descrito acima e conforme sugerido por Bohm e Gimenes (2008), por exemplo.

A Etapa I foi encerrada, a partir do momento em que se teve uma conceituação bem definida do caso, respeitando-se um mínimo de seis sessões. No caso de Dora, diversas dificuldades foram encontradas na definição de uma conceituação de caso e no decorrente preparo das folhas de registro. Desse modo apenas na $25^{\mathrm{a}}$ sessão realizada com a cliente deuse início a Etapa II, com o delineamento experimental. No caso de Sol, a Etapa I foi bem mais curta, com maior facilidade na conceituação de caso e criação da folha de registro, tendo a Etapa II se iniciado na $11^{\mathrm{a}}$ sessão. A conceituação de caso e as respostas definidas para serem utilizadas nas folhas de registro serão descritas abaixo. 


\section{1) Conceituação de caso - caso Dora}

Aspectos relevantes da história e rotina de Dora

Dora, cerca de 40 anos, é mãe de dois filhos e mora sozinha com as crianças. Quando morava com o pai delas (C.), sentia-se frequentemente humilhada e ofendida por ele, tendo sido uma relação permeada por brigas e discussões. Atualmente Dora sente pouco apoio de C. Ele ajuda com algumas contas e com momentos pontuais da rotina do filho mais velho, mas Dora não pode contar muito com ele pois deixa de cumprir com a rotina quando eles se desentendem ou, por exemplo, marcando viagens para fazer sozinho e avisando-a na véspera.

Dora não é próxima de sua família, com exceção de uma tia e guarda certa mágoa de seus pais, por se sentir também injustiçada, sugada e abandonada por eles. Com essa tia, tem também alguns conflitos, já que ambas têm um humor instável, o que acaba gerando dificuldades na relação, apesar de se apoiarem nos momentos necessários. Dora tem apenas uma amiga mais próxima, com quem trabalhava e com quem divide assuntos mais pessoais.

Era frequente, no começo da terapia, Dora reagir de forma agressiva com todos que lhe remetessem a situações de humilhação e injustiças. Frequentemente tem explosões de agressividade tanto em seu trabalho, como em sua vida pessoal.

No mais, sente-se bastante sozinha e com grandes dificuldades em organizar sua rotina, trabalhar, cuidar dos filhos e da casa. Tem dificuldades em cumprir horários e vive constantemente com a sensação de estar atrasada e estar sendo não suficiente em nenhum aspecto da sua vida.

\section{Definições de CCRs e Os de Dora}

O1: dificuldade na forma de colocar limites, de impor respeito, de solucionar conflitos/problemas, de discordar. De forma geral acaba por reagir de quatro formas:

1) agride verbalmente os demais, com a função de calá-lo diante de uma briga ou situações conflituosas;

2) cala-se e faz coisas contra sua vontade ou acompanhadas de sentimento de culpa;

3) desvia o assunto com piadas, ironias ou autocríticas, esquivando-se de resolver a situação e gerando incômodos posteriores, como ficar "ruminando" a situação (pensando repetidamente sobre o assunto e como deveria ter agido, por exemplo);

4) Respostas com a função de aliviar a raiva dirigidas a outros objetos ou pessoas que não a fonte da raiva, como comendo doces, por exemplo. 


\section{CCR1:}

A) dificuldade na forma de colocar limites em sessão, de solucionar conflitos/problemas, de discordar da terapeuta. De forma geral, acaba por reagir de duas formas:

1) por oposição, através de discordância, julgamento ou avaliação desfavoráveis a respeito de afirmações, sugestões, análises ou outros comportamentos emitidos pelo terapeuta;

2) por esquiva: cliente não responde, muda de assunto, ou responde rapidamente voltando/mudando de assunto, tentativas de esconder/editar irritação ou tristeza.

B) emissão de respostas sob controle de estímulos antecedentes que geram respostas com consequências aversivas, sendo que, caso outra propriedade do estímulo antecedente assumisse o controle, respostas sem consequências aversivas seriam evocadas (para mais detalhes sobre esse repertório ver Meyer, 2000). Tal repertório acaba sendo apresentado como formas de ruminar em sessão:

1) expressa em sessão que a terapeuta ou outro a está atacando, julgando, criticando, mentindo para ela ou virá a fazer isso, sem que haja indícios claros no ambiente para essas hipóteses;

2) faz críticas não fundamentada: descreve predominantemente o lado negativo das pessoas, de si mesma e/ou situações de forma exagerada e/ou sem detalhamento/fundamentação da situação (ex. reclamações, julgamentos, preconceitos);

3) afirma que uma expressão de raiva foi inadequada, mesmo tendo sido uma expressão assertiva ou não reconhece suas melhoras.

\section{CCR2:}

A) Colocar limites e expressar sentimentos (de raiva, tristeza, carinho) de forma direta e clara.

1) assertividade: falar se está incomodada com algo da fala da terapeuta ou de outros, estabelecendo limites; fazer pedidos claros e justificados à terapeuta (pedidos de reasseguramento, aprovação, atenção foram consideradas formas de pedido assertiva, ao longo do período de pesquisa);

2) exposição: relatos que indicam que está sob controle das consequências diretas de suas ações e não sob controle primordial de aprovação por outras pessoas.

B) fala de situações nas quais esteve sob controle de contingências que gerassem respostas sem consequências aversivas. Tal repertório foi apresentado como: 
1) análises e críticas fundamentadas: descreve a situação, os outros ou si mesma explicitando os fatores que geram sentimentos negativos e crítica, levando em consideração vários aspectos da situação, não apenas o estar sendo julgada ou julgando ou reclamando (p. ex, quando consegue gerar empatia no ouvinte);

2) afirmação de que uma expressão de raiva foi adequada, quando foi uma expressão assertiva, ou reconhecimento de outras formas de melhora;

3) demonstrações de reflexões sobre o que poderia fazer diferente ao invés de reclamar de si e dos outros e das situações (repertório de solução de problemas).

O2: relatos de situações nas quais foi assertiva:

1) emite respostas apropriadas de resolução de conflitos com colegas, amigos, parentes, filhos, etc.,

2) emite respostas sob controle das consequências diretas de suas ações ao invés de sob controle da audiência, como aceitar ajuda dos demais ou acolhê-los quando necessário sem violar seus direito e vontades para retribuir e sem relatar sentimento de culpa.

Desde a primeira sessão realizada com a cliente, ela estava ciente de que teria que registrar seu comportamento para a coleta de dados da presente pesquisa, mas apenas por volta da $17^{\mathrm{a}}$ sessão, terapeuta e cliente passaram a discutir de forma mais ativa e direta quais os comportamentos Dora deveria registrar e a forma que deveria ser realizado tal registro. Isso porque algumas dificuldades foram encontradas inicialmente na definição de seus CCRs, sobre a forma de conduzi-los em sessão e sobre o paralelo desses CCRs e os comportamentos problema fora de sessão. Apenas na sessão 19, a cliente entregou o registro pela primeira vez e relatou diversos incômodos em preenchê-lo durante a semana. Nas cinco semanas seguintes, entregou-o apenas mais duas vezes. Ao longo desse período, adaptações foram feitas no registro a fim de deixa-lo menos aversivo para a cliente, mas seus incômodos nunca deixaram de existir durante todo o procedimento. A folha de registro foi constantemente tema da sessão para Dora, que frequentemente mencionava o quanto esta era aversiva, mas a auxiliava a refletir sobre algumas questões de seu dia a dia. Apenas na $25^{\mathrm{a}}$ sessão foi possível ter uma maior confiança na regularidade e sistematicidade da anotação de Dora e iniciou-se portanto a Fase A do delineamento. A instrução era a de que o registro deveria ser preenchido diariamente, mas tornou-se frequente a cliente chegar à sessão sem o registro preenchido e pedir licença à terapeuta para preenchê-lo na hora, de acordo com o que lembrava do que tinha ocorrido ou 
até mesmo modificando-o ao longo da sessão conforme relatava algo que a fazia lembrar de algum registro a mais que deveria ter realizado. Nessas ocasiões, de forma geral, a cliente justificava que não havia preenchido o registro anteriormente por falta de tempo (devido sua pesada e corrida rotina) e também pela aversividade do mesmo.

Inicialmente estavam sendo registradas diversas respostas da cliente definidas em conjunto com ela que poderiam ocorrer diante de situações nas quais sentia emoção intensa. Diante das dificuldades e aversividade do registro e do fato de não serem necessárias tantas respostas registradas, reduziu-se o registro por frequência para apenas três respostas que ocorressem nessas situações de eliciação de emoções intensas: Agressão, Agressão não dirigida e Pensamento ruminativo ${ }^{7}$. Essa última foi acrescentada apenas na Fase B1 a pedido da cliente e diz respeito a repassar de forma encoberta a situação e suas respostas diante dessa; em geral eliciando mais sentimentos desagradáveis e não levando a uma resolução da situação. A folha de registro pode ser observada no Apêndice A.

Para o observador externo de Dora, optou-se por manter as diversas respostas levantadas inicialmente com a cliente antes da redução realizada em sua folha de registro (Diante de situações que eliciem emoções intensas, a reação foi de: Agressão, Situação conflituosa não foi aversiva, Esquiva do conflito, calando-se, Assertividade, Agressão não dirigida e Emissão de respostas sob controle das consequências diretas de suas ações (e não da audiência) ${ }^{8}$. Essa folha de registro pode ser observada no Apêndice B.

\section{2) Conceituação de caso - caso Sol}

\section{Aspectos relevantes da história e rotina de Sol}

Sol tem cerca de 30 anos e mora com sua tia. Foi casada por alguns anos e sentia-se amada por seu marido. Seu casamento terminou por ele ter se envolvido com outra mulher, e ter saído de casa repentinamente, conforme conta. Na ocasião ficou emocionalmente muito abalada e depressiva e a família achou melhor que ela fosse morar com uma tia. No início da terapia, mantinha relações bastante abusivas com mãe, tia e namorado, fazendo todas as vontades deles e se anulando quase por completo. Em alguns momentos se incomodava com essas relações e acabava agindo de forma agressiva e impulsiva na tentativa de colocar limites. Gosta de cuidar e ajudar os outros, mas quase sempre anulava-se para isso mantendo-se nessas relações, nas

\footnotetext{
${ }^{7}$ A linguagem utilizada com a cliente e nas folhas de registro foi: Estourar, Usou válvula de escape, Pensamentos desorganizados.

${ }^{8}$ A linguagem utilizada na folha de registro do observador externo foi: Estourou, Não se importou, Se calou, Foi assertiva, Usou válvula de escape, Fez coisas que teve vontade.
} 
quais todos lhe exigiam muito, e lhe davam muito pouco em troca. Conta agir dessa forma com a mãe desde que seu pai abandonou a família, quando ela ainda era criança. Apesar de morar bastante perto do pai desde então, não mantém contato com ele, sendo que certa vez ele nem mesmo a reconheceu na rua.

Procurou por terapia depois de ter vivido uma situação extrema com o ex-marido. Sol conta que por muito tempo sofreu agressões verbais e ameaças da nova esposa dele e nunca reagiu a essas agressões. Porém, certa vez, depois de uma reação explosiva contra essa mulher, que chegou a envolver queixas na polícia, Sol decidiu procurar ajuda terapêutica.

Sol sentia-se bastante sozinha e triste no início da terapia, além de sentir muita ansiedade, em especial a questões relacionadas com essa situação que passou com a esposa do ex-marido e em relação a seu peso. É bastante exigente consigo mesma e com os demais.

\section{Definições de CCRs e Os de Sol}

\section{O1:}

\section{A) Esquiva:}

1) Cala-se, age passivamente ou deixa de expor problemas ou de se defender quando necessário;

2) Expõe-se de forma agressiva, ou irônica;

3) Faz coisas por outras pessoas ou não se recusa a fazê-las, em detrimento de suas vontades e necessidades.

B) Tristeza/Desesperança/Impotência:

1) Relatos de respostas de esquiva de entrar em contato com sentimentos de tristezas;

2) Relatos de respostas impulsivas frente a situações de ansiedade;

3) Relatos de desistências de seus planos.

C) Inflexibilidade/Perfeccionismo:

1) Relatos de irritação quando não fazem as coisas do jeito que ela gostaria;

2) Relatos de irritação ou insatisfação quanto a suas próprias atitudes ou seu próprio corpo.

\section{CCR1:}

A) Esquiva. Frente a perguntas ou solicitações relacionadas diretamente à relação terapêutica ou outros temas: fica em silêncio ou responde de forma curta (monossilábica ou próxima disso); desvia de assunto, não responde suficientemente à questão. 
B) Tristeza/Desesperança/Impotência:

1) Falas de vitimização, tristeza, desesperança, impotência, e/ou pessimismo, não seguidas de análises nem de metas (mesmo nas falas seguintes);

2) Mostra-se conivente, em sessão, com abusos e/ou desrespeitos contra ela ou minimiza essas situações.

C) Inflexibilidade/Perfeccionismo:

1) Autocrítica, demonstrações de culpa, intolerância e/ou incompreensão de suas limitações e/ou quando não faz as coisas perfeitas;

2) Crítica, intolerância e/ou incompreensão com dificuldades dos outros.

\section{CCR2:}

A e B) Enfrentamento, contato e expressão de sentimentos relacionados à relação terapêutica ou não:

1) Expõe como se sente ou o que pensa espontaneamente ou em resposta a perguntas ou solicitações;

2) Faz pedidos, perguntas ou solicitações à terapeuta;

3) Põe limites e expõe sua opinião para a terapeuta, mesmo discordando desta.

4) Falas relacionadas a enfrentamento das situações;

5) Estabelece metas/planos futuros;

6) Estabelece relação entre mudanças dos demais (geralmente positivas) e seus comportamentos;

7) Discordar ou criticar pessoas que a abusaram ou desrespeitaram.

C) Flexibilidade/Aceitação: aceitação, tolerância e/ou compreensão de suas limitações e imperfeições, bem como das dos outros sem ser conivente com abusos e/ou desrespeitos contra ela.

O2:

A e B) Enfrentamento, contato e expressão de sentimentos:

1) Expõe problemas a amigos;

2) Explica ter se calado em situações nas quais acredita que seria punida em caso de exposição;

3) Aceita a ajuda de amigos ou familiares; 
4) Responde à mãe, familiares ou namorado expondo claramente sua opinião, como se sente ou o que deseja;

5) Relatos que indicam que está sob controle das consequências diretas de seu comportamento e não controle social (para agradar);

6) Aceita convites de amigos ou toma a iniciativa de convidá-los.

\section{C) Flexibilidade/Aceitação}

1) Relato de situações externas na qual demonstrou aceitação, tolerância, compreensão e/ou defendeu suas próprias limitações e imperfeições;

2) Relato de situações externas nas quais demonstrou aceitação, tolerância e/ou compreensão das dificuldades dos outros sem ser conivente com abusos e/ou desrespeitos contra ela.

A conceituação de caso de Sol, bem como um entendimento comum entre terapeuta e cliente sobre os comportamentos a serem registrados, ocorreram de forma muito mais rápida do que com a cliente Dora. Sol começou a registrar seu comportamento já na nona sessão e apresentava muito mais clareza sobre o registro que realizava do que Dora, sendo portanto iniciado o delineamento experimental com ela já na $11^{a}$ sessão. Além disso, para Sol o registro não tinha o mesmo caráter aversivo relatado por Dora e por vezes ela descreveu que o registro a ajudava no dia a dia, já que ficava mais atenta sobre suas atitudes. Os comportamentos definidos para serem registrados externamente por Sol dizem respeito a Respostas reforçadas positivamente, produzindo reforçadores mais diretos a ela (comportamentos de melhora) ou Respostas reforçadas negativamente, ao fazer coisas pelos outros evitando punições (comportamentos problema). Além disso, foi registrada mais especificamente a quantidade de vezes que ela Entrou em contato com reforçadores positivos sociais ou Privou-se deles (Apêndice C $)^{9}$.

\section{2) Etapa II - Delineamento experimental}

Foi adotado o delineamento experimental de caso único, com delineamento de reversão, pois este trata o participante, em diferentes momentos, como base de comparação para seu

\footnotetext{
${ }^{9}$ A linguagem utilizada com a cliente para registros dessas respostas foi fez algo por você, ao invés de fazer pelo outro; fez algo pelo outro, ao invés de fazer por você (ambas consideradas comportamento problema); fez ou deixou de fazer algo para agradar o outro; procurar por amigos, quando tem vontade e não procurar por amigos, mesmo tendo vontade.
} 
próprio comportamento, tal como realizado por Geremias (2014), Mangabeira (2014), Oshiro et al. (2012) e Popovitz (2013). Portanto, o delineamento experimental utilizado com as duas clientes da presente pesquisa pode ser descrito como A-B1-BC1-B2-BC2.

Kratochwill et al. (2013) definiu alguns critérios que deveriam ser seguidos em um delineamento experimental de caso-único a fim de se dar confiabilidade à pesquisa realizada. Um desses critérios diz respeito ao número de sessões realizadas e analisadas por fase experimental, fixando-o em pelo menos cinco sessões, quando trata-se de um delineamento de reversão.

Portanto, a Fase A foi composta por cinco sessões e foi utilizada como base de comparação para as demais fases (Linha de Base), permitindo uma medida do comportamento da terapeuta e do cliente antes da introdução sistemática da FAP. Nas Fases B (B1 e B2), foi introduzida uma variável independente (VI), iniciando-se o uso sistemático da parte experiencial da FAP, de modo que foram utilizadas apenas as Regras 1 a 4 (estar atendo, evocar e consequenciar CCRs e verificar o efeito do comportamento do terapeuta sobre o cliente), verificando-se as possíveis modificações decorrentes no comportamento do cliente dentro e fora de sessão. Nas Fases BC (BC1 e BC2), a parte experiencial da FAP foi mantida e foi acrescentada ainda uma nova variável, a parte analítica da FAP, utilizando a Regra 5 (dar modelo, evocar e modelar o repertório de análise do cliente) e foram verificadas as eventuais modificações nos CCRs do cliente e em seu comportamento fora de sessão.

Nas Fases B, esperava-se observar, um aumento nas emissões de CCR2 em relação à Fase A, indicando o uso adequado e efetivo da FAP. Por sua vez, nas Fases BC, esperava-se observar uma estabilidade na emissão de CCR2 em relação à fase imediatamente anterior, indicando a continuidade do uso efetivo da FAP. Tais fases foram encerradas após oito sessões, desde que os CCRs e registros do cliente não tivessem sofrido grandes alterações em funções de acontecimentos atípicos em sua vida; e desde que a terapeuta estivesse aplicando as regras da FAP de forma coerente com a fase experimental vigente.

Vale salientar que em trabalhos anteriores semelhantes, foram realizadas três (Popovitz, 2013), quatro (Oshiro et al., 2012) ou cinco sessões (Geremias, 2014) por fase experimental. Acreditou-se, porém, ser prudente no presente trabalho aumentar esse número para oito sessões (como em Mangabeira, 2014) nas Fases B e BC já que parte da VD seria observada fora da sessão terapêutica. Foi considerado que possivelmente as mudanças terapêuticas fora de sessão não ocorreriam de forma tão imediata quanto aquelas observadas em sessão, optando-se, então por estender cada fase experimental, para aumentar a chance de melhoras externas serem observadas em função da fase experimental vigente. 
Como apresentado por Kazdin (2002), é crucial para um delineamento de reversão a apresentação de níveis estáveis de comportamento. Avaliações e conclusões sobre a causalidade do tratamento não são claras quando a retirada da variável leva apenas à continuidade do padrão de comportamento que vinha sendo observado ou a uma inclinação dos dados em direção ao observado em linha de base. Desse modo, o autor aponta que é recomendável esperar pela estabilidade do comportamento através do tempo. Uma variável introduzida ou retirada pode levar a uma mudança na frequência de um comportamento, enquanto que mudanças bruscas e repentinas no meio da fase podem indicar a influência de outras variáveis, externas ao delineamento experimental. Na presente pesquisa, portanto, aumentos ou diminuições bruscas na emissão de CCRs levaram ao adiamento do encerramento da fase, enquanto que aumento ou diminuição constantes de uma sessão a outra, observadas ao longo de várias sessões, não. Desse modo, na Fase BC1 da cliente Dora, foram realizadas nove sessões experimentais, ao invés de oito, por ter ocorrido uma queda na emissão de CCR2 durante a oitava sessão, somada a um momento de crise ocorrendo na vida da cliente. Assim optou-se por esperar mais uma sessão antes de ser mudada a fase experimental. Para a cliente Sol, as Fases B e BC foram reduzidas para 7 sessões, devido a certo atraso ocorrido no início da coleta de dados por dificuldade na seleção de clientes.

A partir do início da Fase A até o final da coleta de dados, as clientes passaram a registrar diariamente seus comportamentos através da Folha de Registro construída na Etapa I da pesquisa. Além disso, a cada mudança de fase experimental, o observador externo da cliente Dora também recebeu uma Folha de Registro a ser respondida sobre o comportamento dela. Nessas ocasiões não foi dito à cliente que havia uma mudança de fase experimental, apenas era pedido que o registro fosse preenchido sempre com um intervalo de cerca de dois meses, o que coincidia com a mudança de fase. A EAS-40 foi também aplicada a cada mudança de fase experimental e em momentos intermediários de cada fase.

A seguir, serão descritos de forma detalhada os procedimentos realizados pela pesquisadora-terapeuta em cada fase experimental.

\section{Fase A - Fase de Linha de Base}

Cinco sessões foram realizadas sem que houvesse nenhuma intervenção programada. $\mathrm{Ou}$ seja, intervenções de acordo com a FAP poderiam ser realizadas, mas não o foram de forma sistemática. A única diferença dessas em relação às sessões anteriores (Etapa I), foi o fato de que, a partir dessa fase, as sessões foram filmadas e os comportamentos da terapeuta e das 
clientes foram categorizados após cada sessão (em geral, antes da sessão posterior) através do sistema FAPRS (Callaghan \& Follette, 2008), adaptado conforme apresentado anteriormente. Além disso, as clientes passaram a entregar semanalmente o registro que deveriam estar fazendo diariamente sobre seus próprios comportamentos. Desse modo, foi possível obter uma medida dos comportamentos, antes da apresentação das intervenções programadas.

Ao final dessa fase experimental, a cliente Dora entrou em contato com seu observador externo que já havia sido selecionado por ela. Nesse momento foi reiterada a disposição do observador, que se comprometeu a responder ao questionário com seriedade e respeito, mantendo em sigilo as perguntas e respostas envolvidas. Além disso, a própria cliente explicou ao observador os comportamentos a serem registrados por ele. Nesse momento, o primeiro registro foi solicitado.

\section{Fase B1 - Fase FAP sem análise de contingências (FAP sem Regra 5)}

Na Fase B1, a terapeuta iniciou o uso sistemático da parte experiencial da FAP. Análises funcionais a respeito do comportamento das clientes poderiam ser apresentadas apenas como forma de verificar a ocorrência (Regra 1), evocar (Regra 2), reforçar naturalmente os CCRs do cliente (Regra 3) ou como forma de verificar o efeito reforçador sobre os mesmos (Regra 4). Porém, análises funcionais não deveriam ser apresentadas como forma de analisar a interação terapêutica ocorrida, relacionando-a com os comportamentos emitidos fora de sessão, ou seja, não deveriam ser utilizadas como uma forma de transferir as melhoras que estivessem ocorrendo em sessão para fora da sessão (estratégias de generalização, como denominadas pela bibliografia da FAP - Kohlenberg \& Tsai, 1991; Tsai et al., 2009). Era previsto, portanto, que todas as regras da FAP, com exceção das Regras 5, fossem seguidas pela terapeuta durante a sessão (podendo haver um atraso entre as Regras 3 e 4, como apontado por Weeks et al., 2012; sendo que tal pausa pode até mesmo perdurar até a sessão seguinte).

Desse modo, a terapeuta não tentaria evocar análises no repertório das clientes e caso estas fossem apresentadas espontaneamente pelas clientes, a terapeuta não as reforçaria, mas sim tentaria evocar outros CCRs que pudessem ser trabalhados durante a sessão.

Comparado com a Fase A, dentre todas as falas emitidas pela terapeuta deveriam ocorrer com maior porcentagem de emissão as categorias da FAPRS (adaptadas de Callaghan \& Follette, 2008): Regra 1 (avaliar CCRs e seus paralelos), Regra 2 (evocar CCRs), Regra 3 (responder efetivamente a CCR1 e CCR2) e Regra 4 (verificar o efeito de sua resposta). 
Vale ressaltar, que outras intervenções, além das programadas, poderiam ocorrer, por se tratar de um processo terapêutico. Porém, as descritas acima deveriam ocorrer com uma maior porcentagem de emissão do que vinham ocorrendo na fase anterior.

Fase BC1 - Fase FAP com análise de contingências (FAP completa)

Na Fase BC1, a terapeuta continuaria com o uso sistemático da FAP, como na fase anterior, com a diferença de introduzir na interação análises funcionais como estratégias de transferir os aprendizados de dentro da sessão para fora. Ou seja, o que havia sido introduzido na Fase B1 seria mantido, acrescentando-se uma nova variável, sendo, portanto a fase denominada como BC1, pois não haveria a retirada da variável da Fase B1.

Deveriam então ser apresentadas pela terapeuta análises funcionais sobre 0 comportamento das clientes, com objetivo de modelar no repertório delas a emissão dessas análises. Além disso, deveriam ser preferidas as análises sobre a interação ocorrida previamente em terapia (na mesma sessão ou em sessão prévia), relacionando-a com o ambiente externo da cliente. Ou seja, deveriam ser trabalhados através das Regras 1 a 4 da FAP os CCRs das clientes e estes seriam analisados, identificando-se de forma explícita quais as consequências de tais CCRs e fazendo paralelos dessas interações com os comportamentos do cliente fora de sessão. Outras análises, ainda que funcionais, emitidas pelo terapeuta ou cliente que não fossem sobre os comportamentos problema ou de melhora das clientes não seriam consideradas como Regra 5, CCR3 ou O3 na presente pesquisa.

Assim, de modo geral, havia três formas de se emitir Regra 5, diferenciando essas fases das demais: 1) emitindo tais análises de forma fluída e natural na sessão; 2) após uma quebra natural da parte experiencial da FAP; 3) forçando uma quebra ao final da sessão caso análises ainda não tivessem sido feitas de forma natural. Essa última não deveria ser realizada no caso de tal quebra ser muito aversiva ou em caso de interferir significativamente em uma sequência de emissões de CCR2s e Regra 3. Nesse caso, deveria ser esperado para a emissão da Regra 5 na sessão seguinte.

Desse modo, ao longo dessa fase experimental, as mesmas categorias da FAPRS deveriam ser emitidas ao longo da sessão com porcentagem de emissão semelhante à ocorrida na Fase $\mathrm{B}$, mas também as categorias referentes à modelação ou modelagem de análises (Regra 5) passariam a ser emitidas, devendo ocorrer com maior porcentagem de emissão do que na fase anterior. 
A categoria Regra 5-H, que diz respeito à atribuição de tarefa de casa, não deveria ser utilizada nessa fase (como em nenhuma outra fase experimental), pois solicitação de tarefas de casa está relacionada na literatura com estratégias para transferir os ganhos ocorridos em terapia para fora de terapia (Tsai et al., 2009). Portanto, se fossem solicitadas diretamente tarefas de casa às clientes, não seria possível verificar a possível causalidade entre o repertório de análise do terapeuta e cliente e a possível melhora do mesmo fora de sessão.

Assim como na fase anterior, outras intervenções, além das programadas, poderiam ocorrer, por se tratar de um processo terapêutico.

Em seguida, foram repetidas as Fases B e BC (B2 e BC2), seguindo os mesmos critérios acima estabelecidos. Na Tabela 7, é possível observar toda a Etapa II do delineamento experimental.

Tabela 7: Delineamento experimental (Etapa II).

\begin{tabular}{ccccc}
\hline & \multicolumn{5}{c}{ Fase } & \\
\hline A & B1 & BC1 & B2 & BC2 \\
& FAP sem Regra 5 & FAP completa & FAP sem Regra 5 & FAP completa \\
\hline $\begin{array}{c}\text { Ausência de } \\
\text { intervenções } \\
\text { programadas }\end{array}$ & Introdução das & $\begin{array}{c}\text { Manutenção das } \\
\text { Regras 1 a 4 }\end{array}$ & $\begin{array}{c}\text { Manutenção das } \\
\text { Regras 1 a 4 }\end{array}$ & $\begin{array}{c}\text { Manutenção das } \\
\text { Regras 1 a 4 }\end{array}$ \\
\cline { 2 - 5 } $\begin{array}{c}\text { Ausência de uso } \\
\text { sistemático da } \\
\text { FAP }\end{array}$ & Regras 1 a 4 & Introdução da & Retirada de Regra & Reintrodução da \\
Regra 5
\end{tabular}

\section{3) Etapa III - Encerramento da coleta e continuidade da terapia e sessão de} acompanhamento (follow-up)

Ao final do delineamento experimental, as clientes foram consultadas a respeito de seu interesse ou não de dar continuidade à terapia. Na ocasião, ambas as clientes expressaram esse interesse, tendo sido então dado continuidade ao processo terapêutico.

As sessões de terapia após o final da coleta de dados continuaram a ser gravadas em áudio e, nessa ocasião, a terapia continuava em vigor, mas sem o uso sistematizado da FAP, semelhante ao que foi realizado nas sessões da Fase A. Uma dessas sessões de terapia realizada cerca de seis meses depois de finalizada a coleta de dados foi escolhida ao acaso para ser categorizada, a fim de se verificar se houve manutenção dos comportamentos das clientes desenvolvidos ao longo da pesquisa/terapia (sessão de follow-up). Além disso, depois de nove a dez meses de encerrada a coleta de dados o instrumento EAS-40 foi também aplicado 
novamente a fim de se obter mais uma medida a respeito da manutenção dos ganhos terapêuticos.

\section{Análise de dados}

A terapeuta-pesquisadora categorizou seus próprios comportamentos e os comportamentos das clientes, a partir das categorias do FAPRS (adaptado de Callaghan \& Follette, 2008) como apresentado na seção de Instrumentos. Cada resposta categorizada (unidades de análise) correspondeu a fala da terapeuta e das clientes, iniciando-se depois de encerrada a fala anterior e encerrando-se antes de iniciada a fala seguinte. Respostas que apresentassem apenas a função de facilitar a resposta do outro não foram consideradas como uma unidade de análise e não foram categorizadas, assim como proposto por Callaghan et al. (2008).

As cinco sessões gravadas da Fase A e ao menos cinco sessões de cada Fase B e BC, além da sessão de acompanhamento realizada depois de encerrada a coleta de dados foram transcritas pelos transcritores e categorizadas pela pesquisadora. Foram categorizadas ao todo 28 sessões da cliente Dora (11.124 falas) e 30 sessões da cliente Sol (5.954 falas).

Várias análises foram realizadas a partir de tais categorizações sobre o responder da terapeuta em sessão, bem como os CCRs das clientes, como serão apresentadas adiante, na seção de Resultados.

Do mesmo modo, análises sobre os Os emitidos em sessão foram realizadas a fim de se obter a mencionada variedade na forma de coleta de dados externos. Para isso, foi calculada a porcentagem de emissão de falas sobre problemas e melhoras externas realizadas pelas clientes em sessão. Além dessas análises foi feita também uma segunda análise que envolve o relato da cliente em sessão, nomeada aqui como Episódios de O1s e Episódios de O2s. Foi realizada uma contagem sobre os episódios mencionados pelas clientes sobre comportamentos problema e de melhora que ocorreram na semana seguinte à sessão de terapia.

Há algumas características desse dado que o diferenciam dos dados de porcentagem de emissão de Os acima relatados. O primeiro deles é que os anteriores estão sendo relacionados a própria sessão experimental na qual eles são emitidos. Ou seja, se na Sessão 1 da Fase B1 de Dora ocorreram aproximadamente $8 \%$ de emissões de O1, esse dado está sendo atribuído a essa própria sessão. No caso dos Episódios de Os, está sendo considerado que o número de episódios referentes a uma sessão experimental ocorreu depois da mesma, de forma que é preciso contabilizá-los a partir da semana seguinte. Ou seja, se na Sessão 1 da Fase B1 foram 
mencionados 14 episódios de comportamento problema ocorridos na semana, esse dado foi considerado referente a Sessão 5 da Fase A (sessão imediatamente anterior), pois acredita-se que podem ter ocorrido sob influência da condução dessa sessão.

Além disso, há diferenças na forma de contagem desses episódios em comparação com a forma de contabilizar o dado anterior. Imaginando-se uma situação na qual a cliente fala, por exemplo, em cinco momentos separados da sessão sobre uma mesma resposta (considerada como um comportamento problema) que emitiu durante a semana, tal situação geraria uma contagem de cinco repostas de $\mathrm{O} 1$, se não houvesse nenhuma outra categoria do FAPRS envolvida na mesma fala com hierarquia superior a $\mathrm{O} 1$. Na análise que será apresentada a seguir, o episódio narrado é contabilizado apenas uma vez, independente de quantas vezes a cliente falou sobre ele em sessão, gerando um número mais fiel ao que de fato ocorreu durante a semana da cliente.

Ao mesmo tempo, na simples porcentagem de Os, há situações de comportamento problema ou de melhora que podem ter ocorrido durante a sessão e não foram contabilizados nos dados que geraram a porcentagem, já que as categorias de $\mathrm{O} 1$ e $\mathrm{O} 2$ são hierarquicamente inferiores às categorias que envolvem CCRs. Desse modo, episódios de problema ou melhora externa podem ter ocorrido, mas sem ser contabilizados pela fala envolver também um CCR, como já apontado por Busch et al. (2010). Assim, na análise por Episódios de Os, tais episódios foram contabilizados independente de ficarem "escondidos" na hierarquia em uma categorização tradicional realizada com o FAPRS.

\section{Aferição de concordância}

As aferidoras de concordância selecionadas leram o manual do FAPRS (adaptado de Callaghan \& Follette, 2008), a conceituação de caso das clientes e um material sobre o uso das regras da FAP com cada uma das clientes (Apêndice D) e trabalharam na identificação dos CCRs junto à terapeuta. Essa preparação foi feita com uma sessão que não foi utilizada para se avaliar a concordância. Foi planejado que a partir da transcrição da sessão, pelo menos uma sessão de cada fase experimental, dentre as categorizadas pela terapeuta-pesquisadora, seria escolhida ao acaso a fim de ser verificada a concordância da categorização entre a aferidora e a terapeuta-pesquisadora. As aferidoras não seriam informadas sobre qual sessão pertencia a qual fase experimental.

Porém, quando foi realizada a aferição, verificou-se a necessidade de treino adicional, já que os níveis de concordância obtidos foram baixos. Entretanto, terapeuta-pesquisadora e 
aferidoras não puderam disponibilizar tantas horas de treino quanto mencionadas por muitos trabalhos da literatura: cerca de 100 horas gastas por Busch et al. $(2009,2010)$ e Lizarazo et al. (no prelo) e cerca de 38 horas de treino realizado por Popovitz (2013).

A aferidora que apresentou melhores níveis de concordância com a terapeutapesquisadora foi aquela com menos experiência clínica e em FAP (Aferidora 4), mas que conhecia detalhadamente o caso da Sol, por ter transcrito todas as sessões da cliente. No entanto, ela não conhecia a conceituação do caso que a terapeuta havia elaborado durante o período de transcrições; veio a conhecer apenas durante o treino de categorização.

Sendo assim, depois de realizado o treino de categorização, a Aferidora 4 categorizou independentemente três sessões experimentais da cliente Sol, sendo sorteada uma de cada tipo experimental (A, B e BC). As sessões sorteadas pertenciam às Fases A, B1 e BC1, sendo obtido os índices Kappa para clientes de 0,572, 0,617 e 0,476 e para terapeutas de 0,519, 0,519 e 0,364, respectivamente. A maioria desses índices encontram-se abaixo do considerado aceitável $(0,6)$ segundo Callaghan et al. (2008), mas foram considerados suficientes (acima 0,41) de acordo com o critério usado por Popovitz (2013).

As demais aferidoras, ao invés de categorizarem independentemente as sessões sorteadas usaram um método alternativo que não requereria tempo adicional de treinamento. Fizeram uma conferência das categorizações realizadas pela pesquisadora a fim de estabelecer validade às categorizações realizadas. Uma dessas três aferidoras verificou a categorização de três sessões da cliente Sol (Aferidora 3); enquanto as outras duas aferidoras, com grande experiência clínica e em pesquisa em FAP (Aferidoras 1 e 2) verificaram a categorização de três sessões da cliente Dora. O índice Kappa (Siegel \& Castellan, 1988) de concordância entre a categorização das aferidoras e da pesquisadora foi calculado para cada sessão categorizada a partir da formula $x=\left(p_{o}-p_{e}\right) /\left(1-p_{e}\right)$, sendo $p_{o}$ a proporção de concordância observada e $p_{e}$, a esperada de concordâncias ao acaso. Além disso, a porcentagem de concordância foi também calculada. Os valores obtidos podem ser observados na Tabela 8 .

É observado que as concordâncias calculadas a partir da checagem de categorizações apresentam valores bastante altos e bem elevados em relação a concordância calculada para a categorização independente, assim como era esperado. Sabe-se aqui que a forma como foi calculada tal concordância não pode ser considerada ideal, tanto no caso da Aferidora 4 (já que seria desejável obter índices mais elevados), quanto para as demais (que apenas conferiram as categorias, verificando sua coerência ao invés de categorizar independentemente). Porém, ao observar a literatura um pouco mais de perto, verifica-se que várias questões podem ser levantadas a respeito do tema concordância e mais será discutido adiante. 
Tabela 8: Índices Kappa (Siegel \& Castellan, 1988) e porcentagens de concordância entre categorizações das aferidoras e pesquisadora.

\begin{tabular}{|c|c|c|c|c|c|c|c|}
\hline & \multicolumn{7}{|c|}{ Dora } \\
\hline & Aferidora & & $\mathbf{A}$ & B1 & BC1 & B2 & BC2 \\
\hline \multirow{10}{*}{ 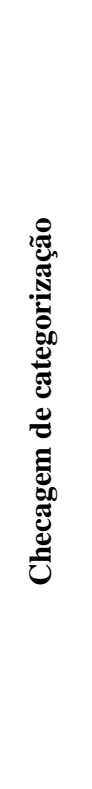 } & \multirow{3}{*}{1} & T (Kappa) & 1 & 0,952 & 0,884 & -- & -- \\
\hline & & C (Kappa) & 1 & 0,99 & 0,905 & -- & -- \\
\hline & & $\%$ geral & $100 \%$ & $99 \%$ & $94 \%$ & -- & -- \\
\hline & \multirow{3}{*}{2} & T (Kappa) & -- & -- & 0,734 & 0,827 & 0,945 \\
\hline & & C (Kappa) & -- & -- & 0,856 & 0,93 & 0,993 \\
\hline & & $\%$ geral & -- & -- & $87 \%$ & $91 \%$ & $97 \%$ \\
\hline & \multicolumn{7}{|c|}{ Sol } \\
\hline & \multirow{3}{*}{3} & T (Kappa) & 0,984 & 0,983 & -- & -- & 0,936 \\
\hline & & C (Kappa) & 0,953 & 0,868 & -- & -- & 0,902 \\
\hline & & $\%$ geral & $97,9 \%$ & $94,6 \%$ & -- & -- & $93,7 \%$ \\
\hline \multirow{3}{*}{ 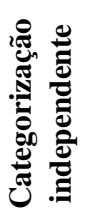 } & \multirow{3}{*}{4} & T (Kappa) & 0,519 & 0,519 & 0,364 & -- & -- \\
\hline & & C (Kappa) & 0,572 & 0,617 & 0,476 & -- & -- \\
\hline & & $\%$ geral & $75 \%$ & $70 \%$ & $62 \%$ & -- & -- \\
\hline
\end{tabular}

\section{Questões éticas}

O estudo foi conduzido com os devidos cuidados éticos, nos termos da Resolução 466/12, do Conselho Nacional de Saúde.

O projeto de pesquisa foi submetido e aprovado pelo Comitê de Ética em Pesquisa com Seres Humanos do Instituto de Psicologia da USP (CEPH-IPUSP), sob o número do processo 07406512.9.0000.5561. O CEPH-IPUSP é credenciado no Conselho Nacional de Ética em Pesquisa (CONEP) e fica localizado na Av. Professor Mello Moraes, no 1721 - Bloco G - Sala 27 - Cidade Universitária - São Paulo/SP. Contatos podem ser feitos pelo telefone (11) 30914182 ou e-mail: ceph.ip@usp.br.

Antes de iniciado o delineamento experimental, as clientes selecionadas foram informadas a respeito da pesquisa a ser conduzida e assinaram o Termo de Consentimento Livre e Esclarecido para Cliente (Apêndice E), autorizando a utilização dos dados obtidos. No caso das clientes selecionadas não concordarem em participar do experimento, as mesmas seriam encaminhadas a outro terapeuta e novos clientes seriam selecionados. 
O observador externo escolhido pela cliente Dora foi convidado pela cliente a participar da pesquisa, assinando o Termo de Compromisso e Responsabilidade para Observadores (Apêndice F). A pesquisadora-terapeuta e as aferidoras de concordância assinaram um Termo de Compromisso e Responsabilidade (Apêndice G), comprometendo-se a garantir o sigilo dos dados, assim como os transcritores de sessões (Apêndice H).

Nesse momento e em todas as futuras ocasiões de divulgação dos dados da pesquisa, seja em apresentações ou publicações, os dados de identificação das clientes não serão divulgados, mantendo-se o sigilo dos mesmos, através da omissão de nomes, locais, cidades e outros dados pessoais que possam ser específicos do caso e que poderiam permitir a identificação das clientes.

O pesquisador foi responsável pelo andamento da pesquisa, aplicação dos procedimentos e avaliação do bem-estar das clientes durante o andamento da coleta de dados. Se tivesse sido identificado qualquer problema e/ou desconforto do cliente em função da pesquisa, a terapeutapesquisadora conversaria com a cliente e ela teria a liberdade de interromper a pesquisa a qualquer momento. Nesse caso, as filmagens das sessões de atendimento seriam suspensas e os dados obtidos até aquele momento destruídos, se assim fosse da vontade da cliente. 


\section{RESULTADOS E DISCUSSÃO}

Frente aos dados coletados, os resultados encontrados com as duas participantes da pesquisa serão apresentados e discutidos em diferentes tópicos. Primeiramente serão (A) focados os dados que indicam a condução do delineamento experimental previsto pela terapeuta-pesquisadora ao longo das diferentes fases experimentais e os resultados gerais apresentados pelas clientes dentro da sessão terapêutica; esses dados serão discutidos em relação àqueles apresentados pela literatura. Em seguida, (B) serão analisados e discutidos os dados coletados sobre os efeitos da variável independente (VI) da pesquisa, a Regra 5, sobre os comportamentos das clientes dentro de sessão. Serão, então, (C) apresentados e discutidos os dados coletados sobre o que ocorreu fora da sessão terapêutica, baseando-se nos indicativos de possíveis melhoras ou pioras no dia a dia de cada cliente. Enfim, (D) serão analisadas e discutidas as relações entre o que ocorreu dentro de sessão e fora de sessão, observando-se quais os efeitos da VI da presente pesquisa experimental, a Regra 5, sobre a transferência de aprendizagem do que ocorreu dentro de sessão para fora de sessão. Por fim, (E) reflexões adicionais sobre o papel das análises na FAP serão conduzidas.

\section{A) Evidências de condução do delineamento experimental e os resultados gerais apresentados pelas clientes dentro da sessão terapêutica}

$\mathrm{Na}$ seção a seguir serão apresentados os dados que evidenciam o seguimento do delineamento previsto em cada uma das fases experimentais, apresentando-se as emissões das categorias diretamente relacionadas à condução da FAP. Por ser uma abordagem descrita e compreendida funcionalmente, não é possível ter certeza de sua correta condução sem ser analisado também o comportamento do cliente. Desse modo, dados das clientes também serão apresentados evidenciando-se uma adequada condução da FAP. Dados iniciais sobre a Regra 5 serão apresentados nessa seção, mas detalhados apenas posteriormente, já que trata-se da variável independente do experimento e merece maior destaque. Da mesma forma, as análises emitidas pelas clientes (CCR3s), também serão apresentadas posteriormente por tratarem-se de uma das variáveis dependentes da presente pesquisa.

A Figura 1 apresenta as porcentagens das categorias referentes às regras da FAP, dentre todas as respostas emitidas pela terapeuta em cada fase experimental. Isto é, foi calculada a porcentagem de emissão de uma determinada categoria de resposta do terapeuta frente a todas as respostas emitidas por ele na fase experimental, assim eliminam-se diferenças em decorrências de terem havido fases com maior número de sessões do que outras. 
Observa-se que, conforme previsto pelo delineamento experimental há maior emissão de regras da FAP nas Fases Bs e BCs do que na Fase A. No caso de Dora, as regras da FAP foram emitidas com uma porcentagem de 22,87\% em na Fase A e com um mínimo de 43,31\% (na Fase B1) nas demais fases experimentais, dobrando-se, portanto, sua porcentagem de emissão. Na sessão de follow-up, estas foram emitidas com uma porcentagem de 34,82\%, sendo, portanto maior do que na Fase A, porém menor do que nas Fases B e BC. No caso de Sol, a diferença entre porcentagem é menor do que no caso de Dora, mas ainda existente. Com essa segunda cliente, as regras da FAP foram emitidas com uma porcentagem de $48,15 \%$ na Fase A e com um mínimo de 60,22\% (na Fase BC1) nas demais fases. Na sessão de follow-up, a porcentagem de emissão dessas regras foi bastante semelhante àquela apresentada na Fase A. Como observado, foi emitida uma porcentagem maior de regras da FAP com essa segunda cliente do que com a primeira, em todas as fases experimentais.
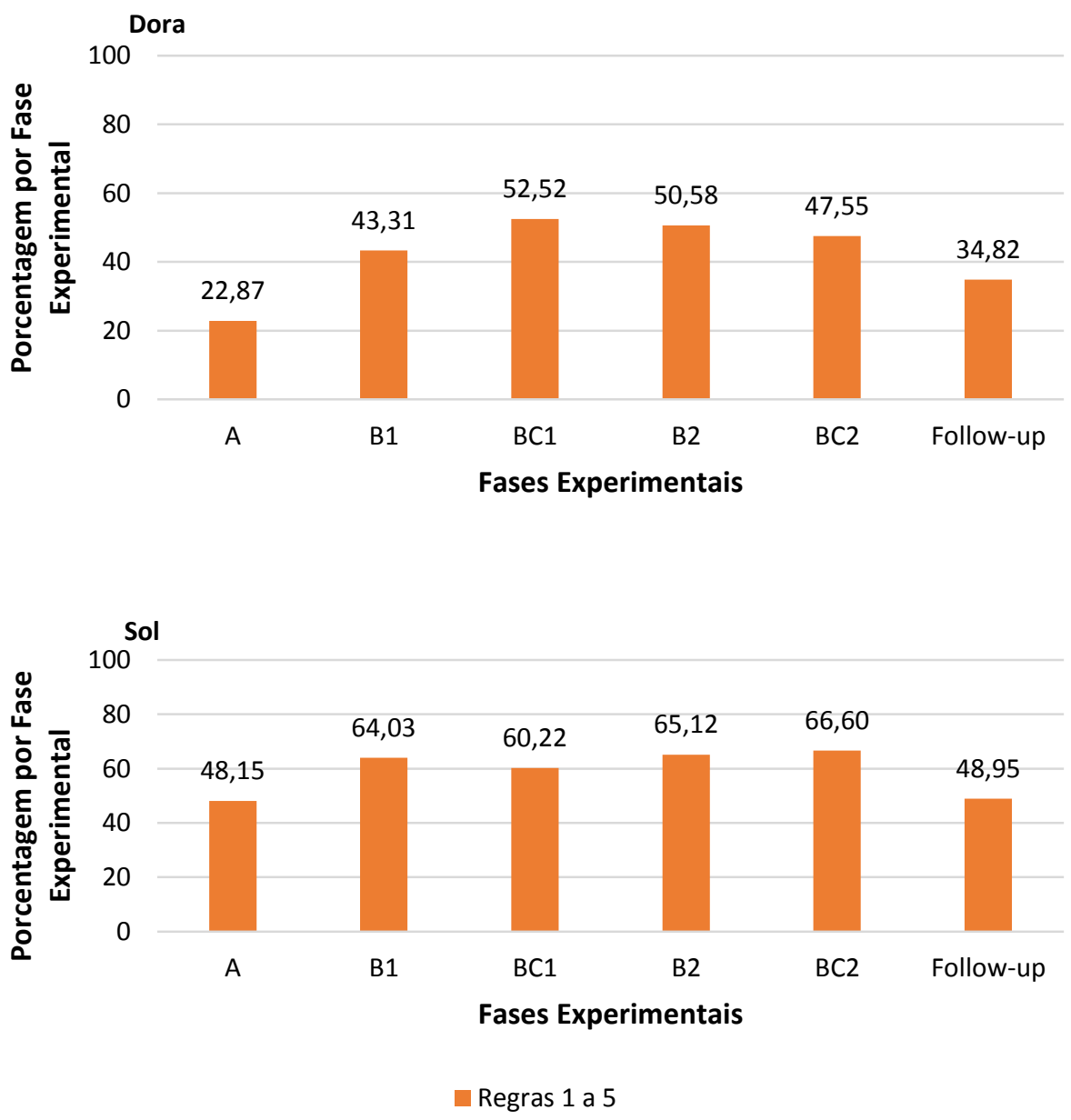

Figura 1: Porcentagem de emissão das categorias referentes às Regras da FAP, dentre todas as respostas emitidas pela terapeuta, por fase experimental e sessão de follow-up com as clientes Dora e Sol. 
Mais detalhes são apresentados na Figura 2 sobre a emissão de regras da FAP em cada sessão experimental e nesse momento, acompanhadas pela porcentagem de emissão dos CCRs (Soma de CCR1s, CCR2s e CCR3) por parte de cada cliente. Por exemplo, foi calculada a porcentagem de emissão de respostas da FAP na sessão, frente todas as categorias emitidas pela terapeuta naquela sessão. Assim, eliminam-se diferenças em decorrências de terem havido sessões com maior número de falas do que outras.

Observa-se que a porcentagem de emissão dos CCRs de ambas as clientes acompanha a emissão das regras da FAP nos dois casos. Foi calculado também o índice de correlação de Spearman $^{10}$ (Siegel \& Castellan, 1988), sessão a sessão, entre emissões de regras da FAP e CCRs para cada cliente, obtendo-se uma correlação de 0,9475 no caso de Dora e de 0,80271 no caso de Sol.
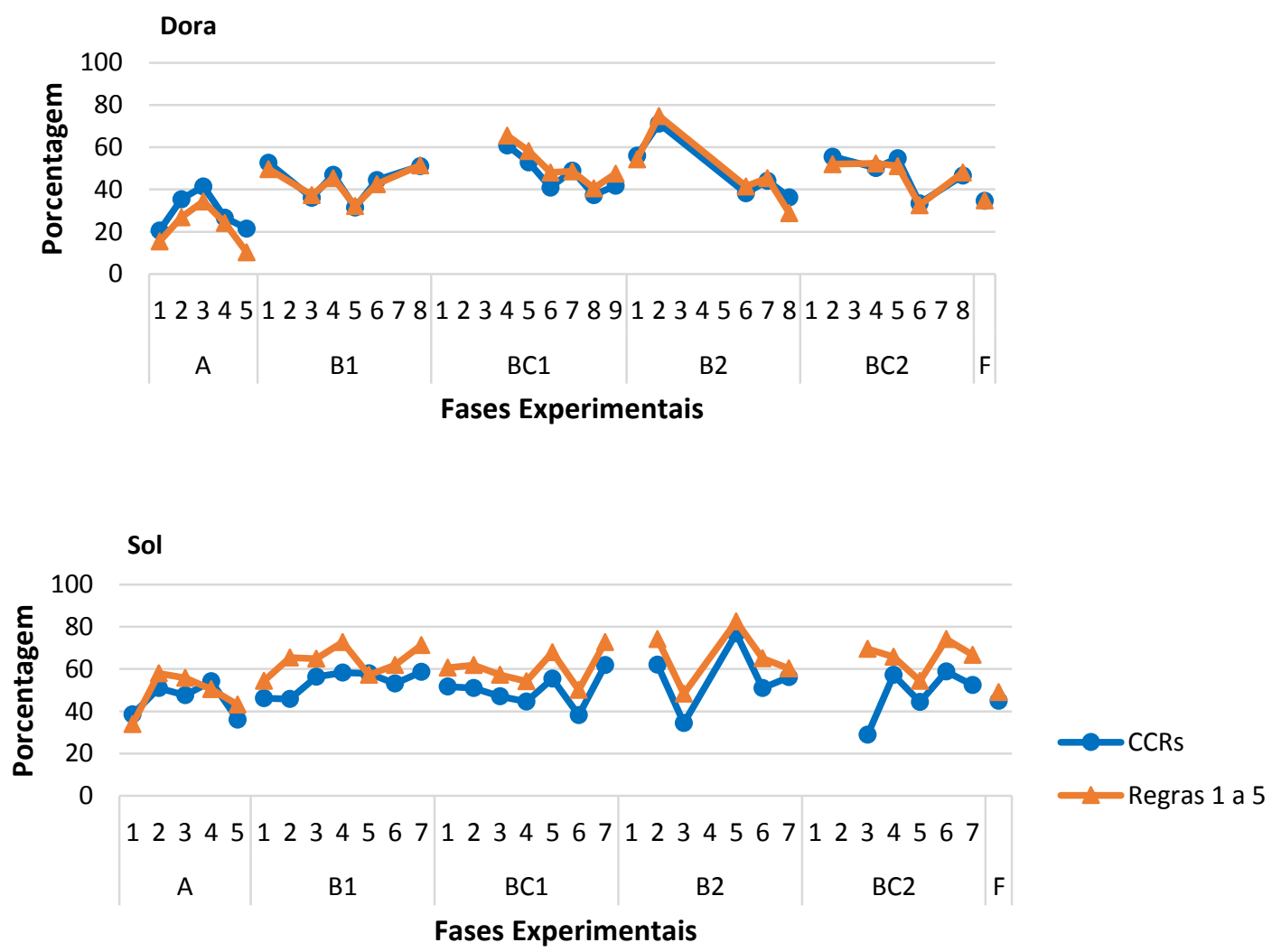

Figura 2: Porcentagem de emissão das categorias referentes às Regras da FAP, dentre todas as respostas emitidas pela terapeuta e porcentagem de emissão de categorias referentes aos CCRs (soma de CCR1, CCR2 e CCR3), dentro todas as respostas emitidas pelas clientes Dora e Sol, ao longo das sessões experimentais $(\mathrm{F}=$ follow-up $)$.

\footnotetext{
${ }^{10}$ As correlações aqui apresentadas, bem como todas as apresentadas adiante foram calculadas sem os dados da sessão de follow-up, que foram obtidos depois dos cálculos da correlação terem sido feitos.
} 
Com a cliente Sol, apesar de serem apresentadas proporcionalmente mais regras da FAP em sessão do que com a cliente Dora, a correlação entre essas regras e os CCRs emitidos por ela é menor. Além disso, é importante mencionar o quanto as regras da FAP já eram frequentes mesmo na Fase A com essa cliente, apresentando uma porcentagem de emissão levemente mais baixa do que a apresentada nas demais fases (Bs e BCs). O conjunto desses dados indica possivelmente uma maior facilidade de emissão de CCRs por parte de Sol do que por parte de Dora, apesar de ter havido maior investimento na FAP por parte da terapeuta com Sol do que com Dora. Pelos dados apresentados não é possível identificar se Sol não respondeu aos comportamentos da terapeuta da mesma forma que Dora ou se a terapeuta é quem emitiu respostas relacionadas a FAP em momento que não caberia a Sol responder a elas. Tal investigação poderia ser feita através de uma análise sequencial das falas de ambas, mas que está fora do escopo do presente trabalho.
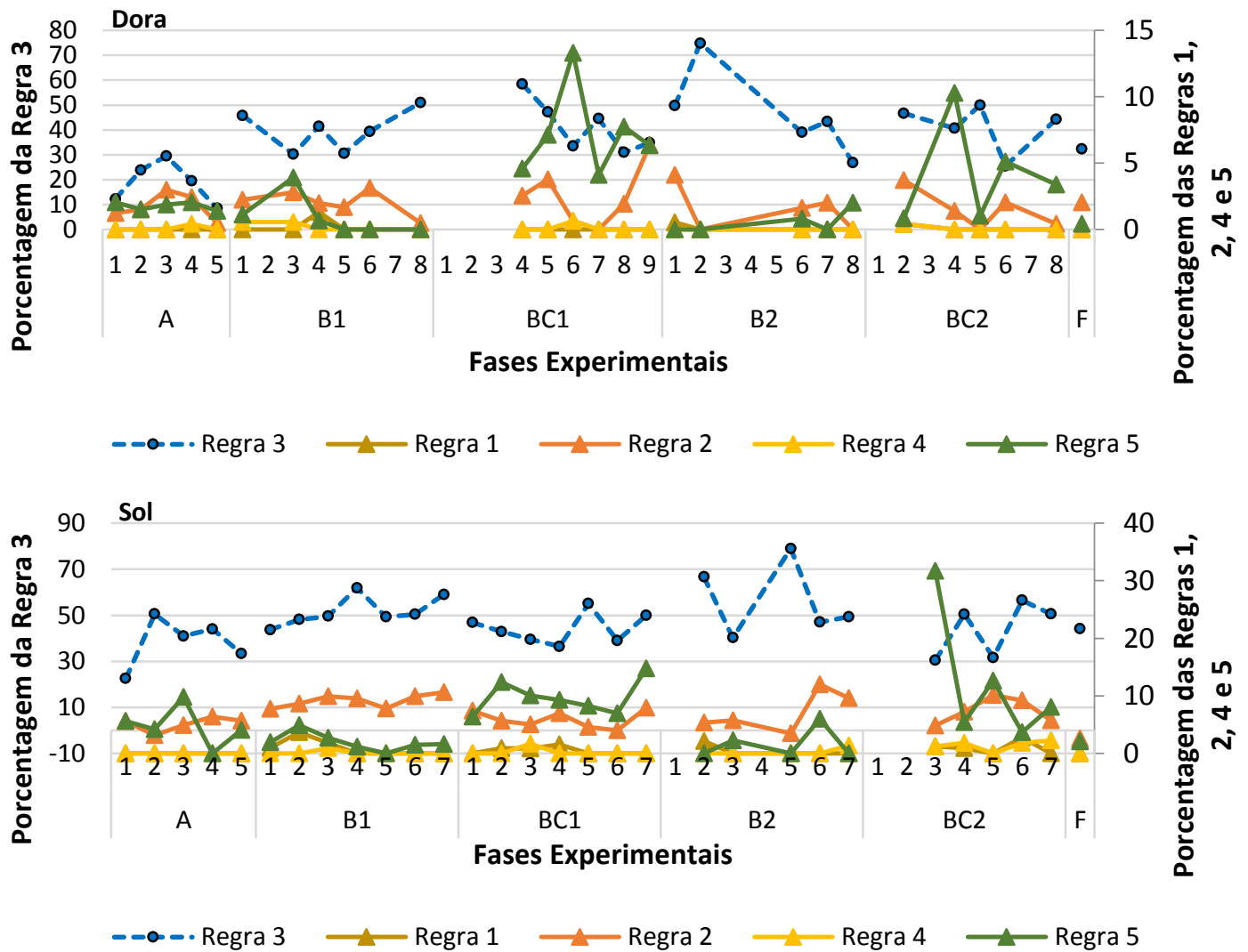

Figura 3: Porcentagem de emissão das categorias referentes a cada uma das Regras da FAP, dentre todas as respostas emitidas pela terapeuta ao longo das sessões experimentais com as clientes Dora e Sol. A Regra 3 encontra-se em uma escala diferente das demais (à esquerda). As escalas dos gráficos são individualizadas para cada participante $(\mathrm{F}=$ follow-up $)$.

Na Figura 3 é apresentado novamente o comportamento da terapeuta, ao longo das sessões experimentais, mas detalhando-se a porcentagem de emissão de cada uma das cinco regras da FAP. Devido a porcentagem de emissão da Regra 3 ser muito elevada em relação às 
demais regras, a figura está sendo apresentada com duas escalas simultâneas, sendo a da esquerda referente à Regra 3 e a da direita, referente às demais regras. Observa-se, portanto, que a Regra 3 é a mais emitida em todas as sessões experimentais analisadas, com exceção da Sessão 3 da Fase BC2 da cliente Sol, na qual a Regra 5 (31,7\%) teve uma porcentagem de emissão levemente superior à Regra 3 (30,5\%).

Ainda a partir da Figura 3, nota-se que, na Fase A, quando a FAP não estava sendo conduzida de forma sistemática, as Regras 2 e 5 se equivaliam em porcentagem de emissão ou se alternavam sobre qual era a mais emitida, para ambas as clientes. Nas Fases Bs, observa-se que a segunda regra com maior porcentagem de emissão, depois da Regra 3, foi a Regra 2, que também é a mais frequente depois da Regra 5 nas Fases BCs. Esses dados condizem com outros trabalhos da literatura que indicam a importância do reforço, mas discutem também o papel da evocação para a efetiva condução da FAP (Busch et al., 2009; Geremias, 2014; Lizarazo et al., no prelo; Mangabeira, 2014; Meurer, 2011; Oshiro et al., 2012), conforme será mais discutido adiante.

Ainda merece destaque o fato da Regra 5 ter sido emitida com maior porcentagem do que a Regra 2 na maioria das sessões analisadas das Fases BCs, o que não ocorre nas Fases Bs. Há sessões nas quais se observam exceções nesse padrão, como na Sessão 3 da Fase B1 e na Sessão 8 da Fase B2 com a cliente Dora, e na Sessão 2 da Fase BC2; com a cliente Sol, as exceções se dão na Sessão 1 da Fase BC1 e nas Sessões 4 e 6 da Fase BC2. Essas exceções possivelmente devem-se ao fato do delineamento ter sido seguido de forma apenas preferencial, como previsto anteriormente, mantendo-se o processo terapêutico como mais importante do que a condução da pesquisa. Além disso, hipóteses mais detalhadas podem ser levantadas observando-se que tais exceções ocorreram com mais frequência e com porcentagem de Regra 5 mais baixa nas Fases BC2, em comparação com BC1, de ambas as clientes. Acredita-se que o desgaste de uma coleta de dados tão longa pode ter afetado na emissão de tais análises. Além do fato de ser exaustivo e possivelmente não terapêutico repetir tantas vezes em sessão as mesmas análises e os mesmos paralelos sobre seus comportamentos com as clientes, tendo essas sido omitidas em alguns momentos ou terem sido realizadas de formas mais pontuais, sem a necessidade de maiores explicações, o que poderia necessitar um maior número de falas.

É também possível observar que nas sessões das Fases BC, nas quais a terapeuta emitiu as maiores porcentagens de respostas de análise, essa porcentagem não ultrapassa 14\% para a cliente Dora e ultrapassa 15\% para a cliente Sol apenas em uma única sessão, chegando a 31,7\% (Sessão 3 da Fase BC2). De forma geral, essa porcentagem de emissão é consideravelmente 
menor do que a porcentagem de emissão da Regra 3 e é bastante semelhante à porcentagem de emissão da Regra 2, que não foi uma variável controlada pelo delineamento experimental. Esses dados podem suscitar a pergunta se a porcentagem de emissão da Regra 5 foi elevada o bastante, dado o delineamento experimental sugerido, ou se ela deveria ter sido emitida com uma porcentagem maior.

Para tal julgamento, pode-se recorrer a dados da literatura que indiquem com qual porcentagem de emissão a Regra 5 tem sido emitida em sessões FAP. Infelizmente, dentre as pesquisas já citadas que realizaram análises intrassessão, apenas dois trabalhos analisaram e divulgaram a porcentagem de Regra 5 emitida, o de Geremias (2014) e o de Lizarazo et al. (no prelo).

Geremias (2014) observa que em fases nas quais a FAP não foi conduzida, não foram observadas emissões de Regra 5 e na fase na qual a FAP foi conduzida, sua porcentagem de emissão não ultrapassou 5\% do total das respostas emitidas pela terapeuta. No caso de Lizarazo et al. (no prelo), a média de porcentagem da Regra 5 em fases nas quais a FAP não foi conduzida, não chegou a $1 \%$ para nenhum dos três clientes $(0,7 \%, 0 \%$ e $0,2 \%)$ e na fase na qual a FAP foi conduzida tal porcentagem se elevou, atingindo $7 \%$ apenas para um cliente $(0,2 \%, 7 \%$ e $0,4 \%)$. O segundo cliente passou também por uma sessão posterior de follow-up, na qual ocorreu $3 \%$ de emissões de Regra 5.

$\mathrm{Na}$ pesquisa de Oshiro et al. (2012) o comportamento da terapeuta apresentado em resposta aos CCRs engloba aquelas que seguem CCR1s, CCR2s e também CCR3s, mas sem englobar análises sobre o comportamento do cliente (para tais detalhamento ver Oshiro, 2011). Desse modo, não é possível se ter acesso isoladamente aos dados referentes às respostas do terapeuta denominadas como Regra 5 na presente pesquisa (envolvendo respostas aos CCR3s e O3, além de exemplos e evocações de análises). São, porém, apresentados dados em Oshiro (2011) de análises das sessões conduzidas a partir da categorização com o Sistema Multidimensional de Categorização de Comportamentos na Interação Terapêutica (SMCCIT, Zamignani, 2007). O SMCCIT permite categorizar (de forma predominantemente topográfica) os comportamentos do terapeuta e cliente de modo a verificar minuciosamente como ocorre a interação terapêutica, permitindo a compreensão de como os comportamentos de um influenciam nos comportamentos do outro, tal como o FAPRS (Callaghan \& Follette, 2008). Assim, Oshiro (2011) verificou que categorias nomeadas como Interpretação e Solicitação de 
Reflexão $^{11}$, emitidas pelo terapeuta, apresentaram porcentagens de 5,26\% e 8,97\% para o primeiro cliente analisado e de 5,6\% e 7,91\% para o segundo cliente, respectivamente. Tais categorias podem ser consideradas como paralelos à Regra 5 conduzida na FAP, com a diferença que estas são mais amplas por incluírem interpretações e reflexões sobre qualquer tema e não apenas relacionados aos comportamentos problema e de melhora dos clientes ${ }^{12}$, de modo que espera-se que tenha sido apresentada com porcentagem mais elevada do que a Regra 5 por si só.

Sendo assim, comparações podem ser feitas apenas em relação aos dados apresentados por Geremias (2014) e Lizarazo et al. (no prelo), de modo que os dados a respeito da porcentagem de Regra 5 apresentados na presente pesquisa são condizentes ou superiores com os apresentados por essas pesquisas. Isso indica que a FAP foi conduzida nas Fases BCs de forma natural, ultrapassando a porcentagem normalmente observada em outras pesquisas em algumas sessões. É bem verdade que mais dados a respeito seriam uma vantagem na investigação de uma quantidade que pudesse ser considerada ideal de emissões de Regra 5 na presente pesquisa, mas na ausência de mais dados, a condução da FAP nas Fases BCs parece condizente com o que era esperado.

Porém, pode-se levantar a hipótese de que talvez dados mais expressivos pudessem ser encontrados caso houvesse uma porcentagem de emissão de Regra 5 maior do que a realizada aqui, mas tal hipótese fica por ora, sem confirmação. No entanto, é importante salientar que o delineamento experimental previsto requeria que as Regras 1 a 4 fossem introduzidas pela terapeuta na Fase B1 e estas fossem mantidas em todas as fases experimentais subsequentes e que a Regra 5 fosse apenas adicionada nas Fases BCs e retirada na Fase B2. Desse modo, não era esperado que houvesse uma redução nas emissões das Regras 1 a 4, de modo a dificultar que uma porcentagem muito maior de Regra 5 fosse emitida.

Os dados apresentados acima indicam que a FAP foi conduzida de forma apropriada, conforme descrita pela literatura e pelo delineamento da presente pesquisa. Porém, resta saber se de fato sua condução resultou nas mudanças esperadas no comportamento das clientes. Serão então analisados, em seguida, a emissão dos CCRs por parte das clientes ao longo do

\footnotetext{
${ }^{11}$ Há ainda dados apresentados sobre a categoria de Recomendação, mas estes não são aqui incluídos por estarem mais relacionados a solicitações de tarefas de casa do que às análises realizadas na Regra 5.

12 Outros trabalhos apresentam ainda a porcentagem de emissão dessas categorias (por exemplo, Kameyama, 2012; Meyer, 2009; Sadi, 2011; Simões-Filho, 2014; Zamignani, 2007), mas não serão aqui discutidos já que as sessões analisadas não correspondem ao modelo da FAP.
} 
delineamento, permitindo-se verificar se as regras apresentadas acima foram funcionalmente definidas.
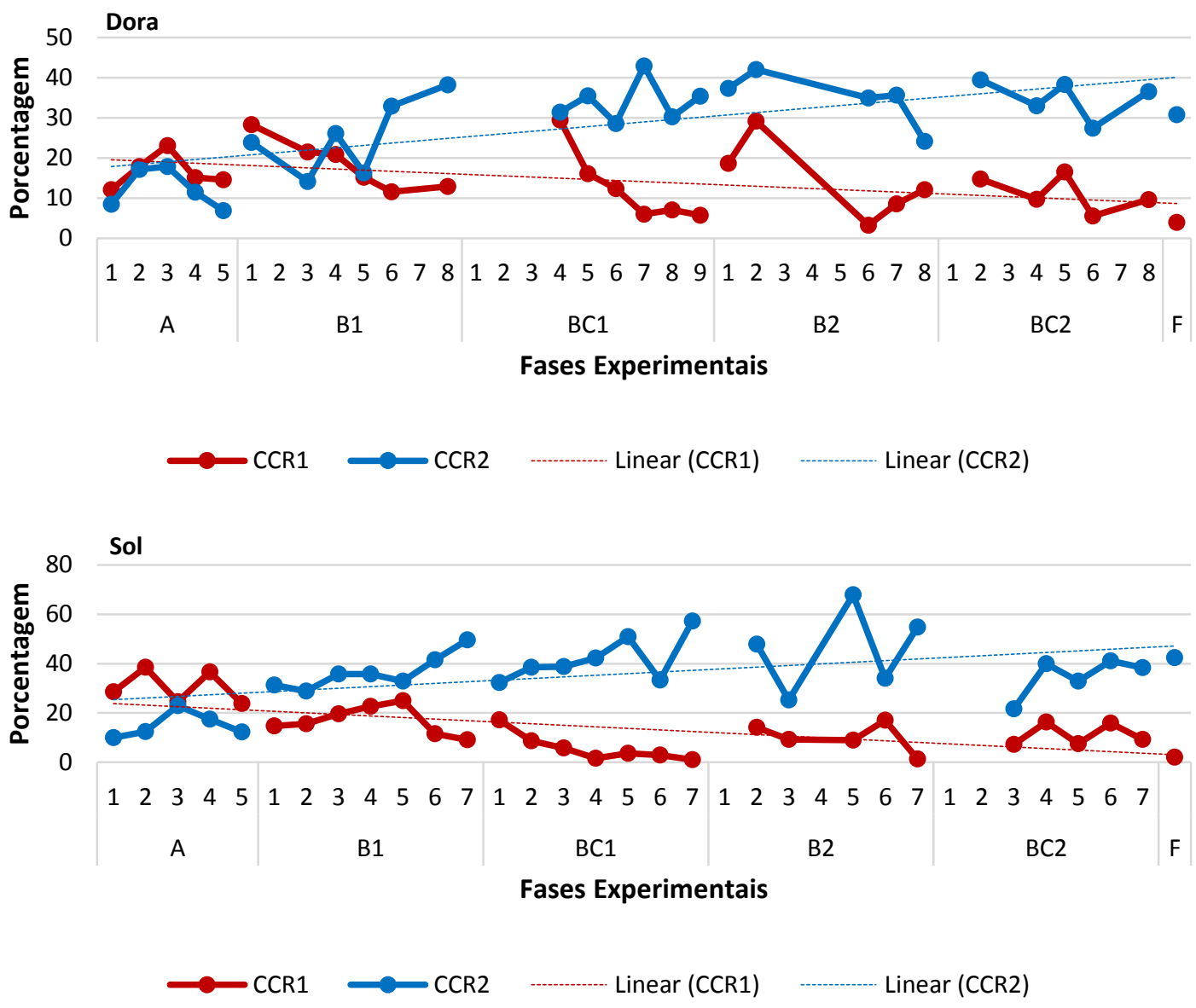

Figura 4: Porcentagem de emissão das categorias referentes à CCR1 e CCR2, dentre todas as respostas emitidas pelas clientes Dora e Sol, ao longo das sessões experimentais. As escalas dos gráficos são individualizadas para cada participante $(\mathrm{F}=$ follow-up $)$.

Na Figura 4 é possível observar que, na Fase A, havia maior porcentagem de ocorrências de CCR1 do que de CCR2 para ambas as clientes, sendo a diferença mais sutil para a cliente Dora do que para a cliente Sol. Ao ser introduzida a parte experiencial da FAP, na Fase B1, essa predominância se inverte nos dois casos. No caso da cliente Dora, a inversão do CCR predominante ocorreu lentamente, apresentando queda na emissão de CCR1 ao longo dessa fase, mas certa oscilação de CCR2 até a Sessão 5. Faz-se interessante apontar que, como observado anteriormente na Figura 2, houve um aumento de emissões de CCRs de $21 \%$ para $52 \%$ da última sessão da Fase A para a primeira sessão da Fase B1, sem grandes alterações na qualidade desses CCRs, ou seja, nessas duas sessões a porcentagem de emissão de CCR1 era levemente superior à porcentagem de emissão de CCR2. Observa-se que a porcentagem de CCR1 foi diminuindo gradualmente ao longo da Fase B1, enquanto CCR2 sofreu um aumento, ainda que oscilante. Após a Sessão 5 dessa fase, CCR2 predominou em relação a CCR1 em 
todas as sessões analisadas da cliente Dora. Além disso, a curva de tendência linear de CCR2 apresenta inclinação maior ascendente do que a inclinação descendente da curva de CCR1, indicando que o aumento de CCR2 ao longo do delineamento ocorreu em maior intensidade do que a diminuição de CCR1.

Cabe aqui apontar que a Fase BC1 da cliente Dora foi realizada com nove sessões experimentais (conforme mencionado na seção de Método), uma a mais do que a prevista inicialmente no delineamento. Isso ocorreu em função da queda na porcentagem de emissão de CCR2 observada na Sessão 8 em relação à sessão anterior. Achou-se mais seguro então prolongar em mais uma sessão tal fase experimental para que não houvesse mudanças de fase diante de instabilidades do comportamento da cliente, conforme previsto anteriormente. Já no final da Fase B2, novamente os CCR2s de Dora voltaram a cair, porém, dessa vez, não prolongou-se a Fase B2 pois entendeu-se que a queda de CCR2 e aumento de CCR1 foram ocasionadas por um "efeito piso", ou seja, na Sessão 6 dessa fase, quase não houve emissões de CCR1 (apenas 3,3\%), e considerou-se natural que depois de uma sessão na qual praticamente todos os CCRs emitidos foram CCR2, esse número cairia nas sessões seguintes, não ameaçando a mudança de fase.

No caso da cliente Sol, a inversão na qualidade de CCRs emitidos ocorreu rapidamente, desde a Sessão 1, da Fase B1. Para essa cliente, a porcentagem geral de CCRs aumentou gradativamente ao longo da Fase B1, tanto em relação ao CCR1 quanto ao CCR2, sendo que a porcentagem de CCR2 permaneceu maior do que a de CCR1 em todas as sessões analisadas até o final do delineamento experimental. Porém vale lembrar que, como evidenciado anteriormente (Figuras 1 e 2), na Fase A realizada com Sol, já havia uma porcentagem de ocorrências de regras da FAP alta e maior do que no caso de Dora. Desse modo, o aumento de regras da FAP que ocorreram da primeira sessão da Fase B1 com essa cliente foi talvez suficiente já para tornar ocorrências de CCR2 mais frequentes do que as de CCR1. A porcentagem de CCRs ocorridas na última sessão da Fase A é próxima da porcentagem ocorrida na primeira sessão da Fase B1, ocorrendo um aumento de cerca de 36\% para cerca de 46\%. No entanto, há grande e repentina diferença na qualidade desses CCRs (1 ou 2), evidenciado pela Figura 4. Duas hipóteses, não excludentes, podem ser levantadas a respeito disso. A primeira é que talvez houve um efeito acumulado da emissão das regras da FAP que resultou em uma mudança apenas no início da fase seguinte, devido ao aumento, ainda que modesto na porcentagem de emissão das regras. A segunda hipótese é de que apesar de regras da FAP estarem sendo emitidas desde as primeiras sessões experimentais, talvez tenha ocorrido uma mudança na qualidade delas, em alguma variável não detectada pelo sistema FAPRS e 
que pode ter sido responsável pela abrupta mudança na qualidade dos CCRs emitidos por Sol. Nos trabalhos de Oshiro et al. (2012), Geremias (2014) e Mangabeira (2014) mudanças abruptas como essa observada nos dados de Sol são também observadas, nas fases nas quais são introduzidas a FAP, sendo esse dado replicado mais de uma vez ao longo do delineamento realizado por esses autores, com sucessivas reversões quanto ao CCR predominante, em cada nova apresentação da FAP.

Ainda sobre essa segunda cliente, vale ressaltar que na Fase B2, ocorreu grande oscilação na porcentagem de emissão de CCR2, chegando a apresentar a menor porcentagem emitida até então (Sessão 3), desde que havia sido introduzida a FAP. Na Fase BC2, tal oscilação diminuiu, mas sua porcentagem de emissão apresentou níveis mais baixos do que nas Fases B e BC anteriores. Esses dados indicam que houve certa piora no comportamento da cliente em sessão nessas últimas fases, mas ainda com emissões de CCR2 superiores e emissões de CCR1 inferiores àquelas apresentadas na Fase A. Além disso, as curvas de tendências lineares apresentam inclinações similares, porém inversamente proporcionais, indicando que o aumento de CCR2 e a diminuição de CCR1 ocorreu de forma similar, ao longo do delineamento.

Como discutido por Busch et al. (2010), variações na quantidade de CCRs emitidos em diferentes sessões são esperadas em uma terapia FAP, já que inúmeros fatores podem influenciar em sua frequência. Os autores apontam que os CCRs podem ocorrer naturalmente ou serem evocados pelo terapeuta, mas esse não segue regras rígidas de como atuar e quanto ao número de CCRs que devem ser evocados. Além disso, inúmeros fatores fora da terapia, no cotidiano do cliente podem ocorrer e influenciar seu comportamento mesmo em sessão, na relação com o terapeuta, fazendo inclusive que algumas classes de CCRs sejam mais emitidas em determinada sessão do que em outras.

No caso de Sol, como mostra a Figura 4, observa-se que emissões de CCRs foram relativamente constantes até a Fase $\mathrm{BC} 1$ do experimento e ao final desta e nas fases seguintes, grande variação começou a ocorrer. À semelhança do que é relatado em Busch et al. (2010), nessas duas últimas fases da terapia de Sol, houve uma mudança no principal tema tratado em sessão. A cliente sempre apresentou problemas com sua imagem corporal, estando constantemente preocupada com seu peso desde o início da terapia, mas falando muito pouco sobre isso por ser um tema extremamente aversivo a ela. Do meio para o final do delineamento experimental, Sol passou a apresentar maior enfrentamento ao falar sobre esses temas diretamente, mas muitas vezes de forma bastante crítica a si mesma, ora emitindo então CCR2s ao não se esquivar de temas difíceis, ora emitindo CCR1s ao ser extremamente crítica consigo mesma em relação a seu peso e desesperançosa sobre ser capaz de mudar. Desse modo, grande 
variação passou a ser observada na porcentagem de emissão de seus CCRs em sessão, em especial do CCR2.

Ainda a partir da Figura 4, faz-se intrigante observar que depois de introduzida a FAP (Fase B1 em diante), observou-se sistematicamente algum dado indicando ao menos uma pequena piora no comportamento das clientes em sessão, na primeira sessão categorizada de cada fase experimental, em comparação com a última sessão da fase anterior. Em especial, fazse importante mencionar o aumento de CCR1 ocorrido com a cliente Dora na primeira sessão da Fase B1, quando foi introduzida sistematicamente a FAP. Assim como já discutido em Busch et al. (2009) aumentos nas emissões do comportamento problema do participante logo após uma mudança de fase experimental podem representar que o novo delineamento adotado levou a uma piora no comportamento do cliente e no andamento da terapia. No entanto, os autores mencionam que esse aumento não é de se estranhar tratando-se da introdução de uma terapia FAP, na qual o terapeuta passa a evocar intencionalmente os comportamentos problema emitidos pelo cliente dentro da sessão terapêutica, sendo que tal evocação não ocorria anteriormente. Porém, na presente pesquisa, pioras no comportamento das clientes não foram observados apenas no início da Fase B1, com a introdução da FAP. Tais pioras são indicadas tanto pelo aumento na porcentagem de emissões de CCR1, como no caso da cliente Dora, como pelo aumento na porcentagem de emissões de CCR1 e concomitante diminuição na de CCR2 no caso da cliente Sol. A primeira sessão realizada em algumas fases experimentais não foi categorizada (Fases BCs da cliente Dora e Fases B2 e BC2 da cliente Sol) e por isso não é possível afirmar que tal piora ocorreu sistematicamente em função da mudança de fase, ou seja, pela mudança na variável manipulada ou outras mudanças não previstas na condução da sessão. Mais investigações, sobre o comportamento da terapeuta no início de cada fase experimental, e em especial nas primeiras sessões categorizadas de cada fase, deveriam ser feitas para uma melhor compreensão desse dado. No entanto, entende-se que tais investigações, necessitam de um maior detalhamento do que o que já foi aqui apresentado e acabam por se encontrar fora da alçada do presente trabalho.

Apesar das mencionadas oscilações na porcentagem de emissão dos CCRs das clientes, é inegável a predominância de CCR2 em relação ao CCR1 durante a maior parte das Fases Bs e BCs de ambas as clientes. Além disso, observa-se que tal predominância se manteve na sessão de follow-up, indicando uma manutenção dos ganhos obtidos, mesmo seis meses após o encerramento da coleta de dados e na ausência de uma condução sistemática da FAP. Em outras palavras, na sessão de follow-up, a terapia foi conduzida de forma semelhante àquela 
apresentada na Fase A, porém, os comportamentos das clientes em sessão mostram-se consideravelmente diferentes do que o que foi apresentado naquela fase inicial.

Pode-se questionar, no entanto, quais comportamentos da terapeuta em sessão levaram a tal resposta no comportamento das clientes. A Figura 5 detalha as emissões de Regra 3 ocorridas ao longo das sessões experimentais, separando-a em TRB1 (respostas ao CCR1) e TRB2 (respostas ao CCR2).
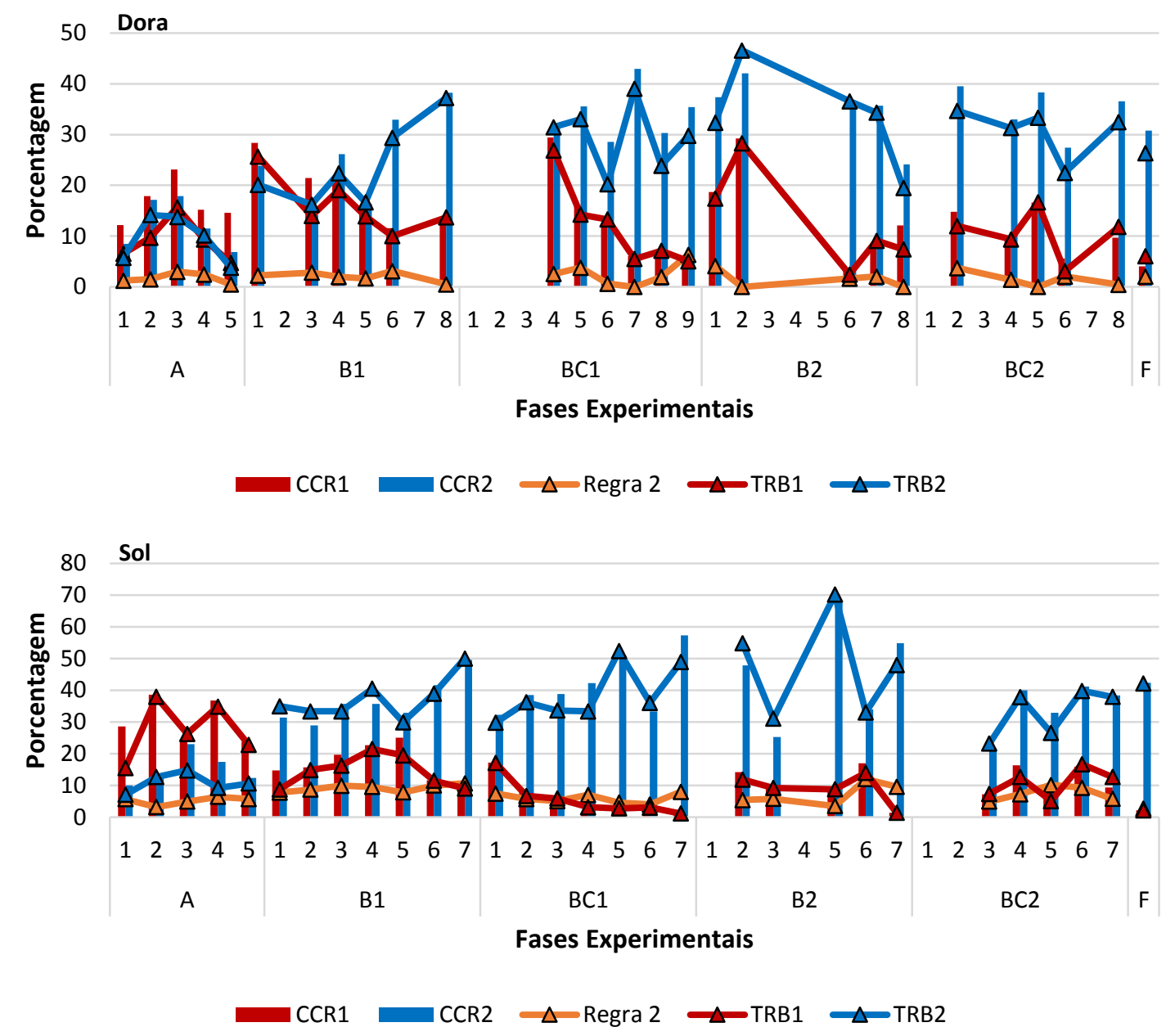

Figura 5: Porcentagem de emissão das categorias referentes à CCR1 e CCR2 (barras), dentre todas as respostas emitidas pelas clientes Dora e Sol e porcentagem de emissão das Regras 2 e 3 (TRB1 e TRB2), dentre todas as respostas emitidas pela terapeuta (linhas) ao longo das sessões experimentais. As escalas dos gráficos são individualizadas para cada participante $(\mathrm{F}=$ follow-up $)$.

Nota-se a evidente relação entre CCR1 e TRB1 e a relação entre CCR2 e TRB2, indicando uma adequada consequenciação dos CCRs da cliente por parte da terapeuta. Tal dados sugere fortemente que o responder da terapeuta foi contingente e contíguo e provavelmente o principal responsável pelas mudanças na porcentagem de emissão dos CCRs. 
A correlação de Spearman foi calculada também sessão a sessão para esses dados e nota-se que para a cliente Dora foi observada uma correlação entre CCR1 e TRB1 de 0,87179 e entre CCR2 e TRB2 de 0,95788; no caso de Sol os valores observados foram de 0,95665 e de 0,90746.

Além disso, observa-se na Figura 5 que, no caso de ambas as clientes, emissões de Regra 2 apresentaram uma porcentagem baixa e constante ao longo de todo o delineamento experimental, não parecendo sofrer alterações de acordo com a emissão de CCRs e nem afetando a quantidade de emissão desses.

Essa alta relação entre as porcentagens de emissão dos CCRs e de suas respectivas Regra 3 replica a importância descrita na literatura da consequenciação da resposta para sua mudança (Follette \& Bonow, 2009; Follette, Naugle, \& Calaghan, 1996; Kohlenberg \& Tsai, 1991; Tsai et al., 2009, 2012). Como já mencionado, outros trabalhos da área investigaram qual o principal mecanismo de mudança na FAP que leva a modificação do comportamento dos clientes em sessão e apresentam indícios de que o responder contingente da terapeuta ao comportamento do cliente pode ter sido responsável pela melhora do cliente apresentada (Callaghan et al., 2003; Busch et al., 2009, 2010; Geremias, 2014; Kanter et al., 2006; Lizarazo et al., no prelo; Mangabeira, 2014; Meurer, 2011; Oshiro et al., 2012; Popovitz, 2013; Xavier et al., 2012).

No presente trabalho, a alta taxa de emissão de Regra 3, seguida pela Regra 2, e as correlações dessas regras com os CCRs levam a reflexões que apoiam essa hipótese. Certamente uma análise sequencial que analisasse a evocação dos CCRs a partir da Regra 2 e a consequenciação deles pela Regra 3 poderia indicar qual das duas teve uma maior influência sobre suas ocorrências, mas novamente considera-se que tal análise está fora do escopo do presente trabalho. No entanto, vale salientar que o próprio sistema FAPRS, se utilizado adequadamente mostra por si só uma análise sequencial ao menos em relação à Regra 3 seguindo os CCRs (pois essa categoria só pode ser registrada em resposta, ainda que não imediata, a CCRs). Apesar de não confirmar, sua alta frequência indica um importante papel dessa regra sobre o comportamento do cliente em sessão.

Por outro lado, Busch et al. (2009) apresentam dados que mostram que nas sessões FAP por eles analisadas foi mais frequente o investimento na melhoria da relação terapêutica e na evocação de CCRs (Regra 2) do que a consequenciação deles (Regra 3), propriamente dita. Oshiro et al. (2012) também chega a discutir que ao menos para um de seus clientes, o papel evocativo da FAP talvez tenha sido importante na reversão dos CCRs observada em fases FAP em comparação com fases não FAP. Esse tema também é discutido por Geremias (2014), Lizarazo et al. (no prelo), Mangabeira (2014) e Meurer (2011). 
Vale também lembrar que a consequenciação aos CCRs apresenta em muitos casos também um caráter evocativo e muitas respostas da terapeuta que apresentam função de Regra 2 (função evocativa) não são assim categorizadas pelo FAPRS por apresentarem também função de Regra 3, ficando então os dados de emissão de Regra 2 subestimados o que não ocorre com as emissões de Regra 3. Mais investigações se fazem necessárias a fim de delimitar o papel de cada uma dessas regras sobre os CCRs. Mas ao menos na forma como o comportamento da terapeuta foi categorizado no presente trabalho, a Regra 3 mostra-se como a grande responsável pelas mudanças de comportamento das clientes dentro da sessão.

O conjunto de dados apresentados acima indica a fidelidade do responder da terapeuta ao delineamento proposto e seus efeitos gerais sobre o comportamento das clientes. Desse modo, conclui-se que a FAP foi conduzida corretamente, levando ao esperado aumento de CCR2s e diminuições de CCR1s ocorridos em sessão em função da condução da parte experiencial da FAP.

Resta ainda saber se as emissões de Regra 5 também causaram efeitos sobre os CCRs das clientes e qual sua importância e relevância em sessões de psicoterapia conduzidas com a perspectiva da FAP. Tal dado será investigado a seguir. Como apontado anteriormente, há duas VD's sendo investigadas na presente pesquisa: 1) o comportamento das clientes dentro de sessão, em especial o de análise sobre seu próprio comportamento e 2) suas eventuais melhoras fora de sessão. A seguir serão então apresentados e discutidos em detalhes os efeitos da Regra 5 (VI) sobre respostas das clientes emitidas em sessão.

\section{B) Os efeitos da Regra 5 sobre comportamentos dentro de sessão}

A seguir serão analisados os dados referentes às emissões de respostas categorizadas como Regra 5, a principal variável da presente pesquisa. Na Figura 3 apresentada na Seção A foi possível observar a porcentagem de emissão de Regra 5 dentre todas as respostas emitidas pela terapeuta em sessão, em comparação com as Regras 1, 2, 3 e 4. Na presente seção, serão apresentados e discutidos os possíveis efeitos e relações da Regra 5 sobre os comportamentos de análise das clientes (CCR3 e O3). Além disso, será apresentada uma análise sobre as possíveis relações entre emissões de respostas de análise (Regra 5, CCR3 e O3) e os demais CCRs (CCR1 e CCR2) a fim de se investigar os efeitos relevantes que as análises do comportamento do cliente pode ter sobre a condução de uma sessão FAP. 


\section{B.1) Regra 5 e as análises das clientes}

B.1.1) Efeitos da Regra 5 sobre as análises das clientes (CCR3 e O3)

Na Figura 6, observa-se que, de forma geral, emissões de respostas de análise por parte das clientes (CCR3 e O3) acompanharam emissões de respostas de análises por parte da terapeuta (Regra 5), havendo uma correlação entre elas de 0,82238 no caso de Dora e de 0,86452 no caso de Sol, conforme mostra a Tabela 9.

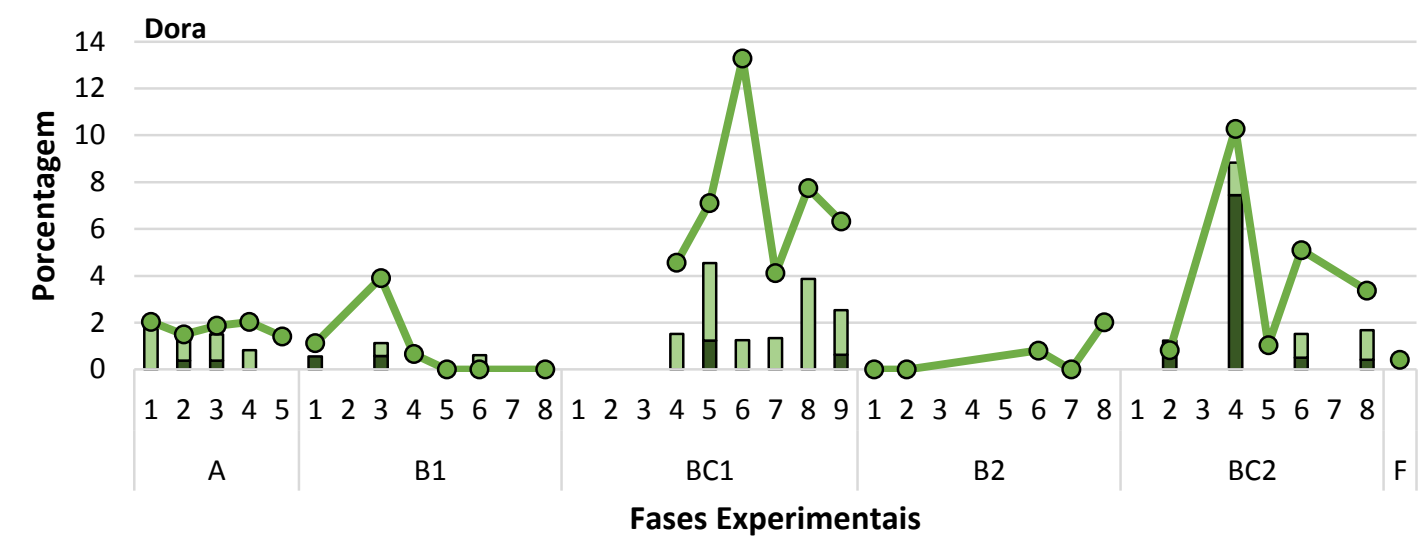

$\square$ CCR3 $\square 03-$ Regra 5

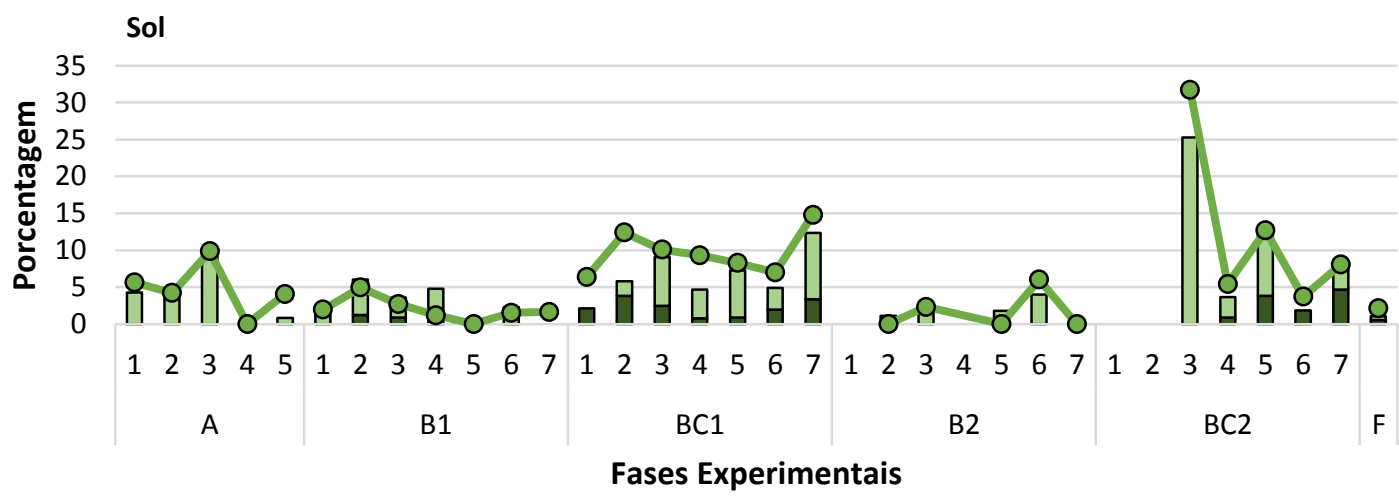

$\square$ CCR3 $\square 03-$ Regra 5

Figura 6: Porcentagem de emissão da categoria referente à Regra 5 (linha), dentre todas as respostas emitidas pela terapeuta e porcentagem de emissão das categorias referentes às análises realizadas pela cliente Sol (soma de CCR3 e O3 - barra), dentre todas as categorias emitidas pelas clientes Dora e Sol, ao longo das sessões experimentais. As escalas dos gráficos são individualizadas para cada participante $(\mathrm{F}=$ follow-up $)$. 
Tabela 9: Correlações de Spearman entre porcentagem de emissão da Regra 5, dentre todas as respostas emitidas pela terapeuta, e a porcentagem de emissão das categorias referentes a CCR3 e O3 dentre todas as respostas emitidas por cada uma das clientes. A correlação foi calculada sem os dados da sessão de follow-up.

\begin{tabular}{ccc}
\hline & Dora & Sol \\
\hline \multicolumn{2}{c}{ Regra 5 } \\
\hline CCR3 & 0,42684 & 0,63008 \\
\hline O3 & 0,81881 & 0,70428 \\
\hline CCR3 + O3 & 0,82238 & 0,86452 \\
\hline
\end{tabular}

Mais detalhadamente, observa-se na Figura 6 que, na Fase A, havia certa quantidade de análises emitidas tanto pela terapeuta quanto pelas clientes e houve uma diminuição das mesmas na Fase B1, quando foram introduzidas as Regras 1 a 4 da FAP. Na Fase BC1 ocorreu a introdução da Regra 5 e um consequente aumento na porcentagem de emissão dos comportamentos de análises emitidos pelas clientes. Em seguida, na Fase B2, a Regra 5 voltou a ser emitida com uma porcentagem mais baixa, semelhante à que ocorria na Fase B1, e observa-se, uma queda na porcentagem de emissão das análises emitidas pelas clientes, chegando a 0\% no caso da cliente Dora. Por fim, na Fase BC2, a variável Regra 5 voltou a ser introduzida, tendo sua porcentagem de emissão novamente aumentada e verificando-se novamente um acréscimo nas emissões de análises por parte das clientes. Tal resultado indica fortemente que emissões de respostas de análises das clientes ocorreram em função das análises realizadas pela terapeuta em cada uma das fases experimentais, já que houve inclusive replicação desse fenômeno ao longo do delineamento. Além disso, observa-se variação na porcentagem de emissões de Regra 5 de uma sessão para outra, sendo acompanhadas por variações semelhantes na porcentagem de emissão de análises por parte das clientes.

É possível ainda observar as porcentagens separadas referentes à emissão de CCR3 e de O3. Emissões de O3 foram, em geral, mais frequentes do que emissões de CCR3, indicando ter sido mais frequente as clientes fazerem análises sobre o que acontecia fora de sessão do que sobre o que acontecia dentro de sessão. Há algumas poucas exceções, na Sessão 1, da Fase B1 e nas Sessões 2 e 4 da Fase BC2 com a cliente Dora; bem como nas Sessões 1 e 2 da Fase BC1 e nas Sessões 6 e 7 da Fase BC2 da cliente Sol. Além disso, pode-se analisar as correlações existentes entre emissões de CCR3 e $\mathrm{O} 3$ com a Regra 5, em separado, como mostra também a Tabela 9. Observa-se que a correlação entre Regra 5 e O3 (análises sobre fora da sessão) foi maior para ambas as clientes do que a correlação entre Regra 5 e CCR3 (análises sobre dentro da sessão), indicando que há maior relação entre análises do terapeuta com análises extra sessão nos dois casos. Além disso, a correlação entre Regra 5 e O3 é maior para a cliente Dora do que 
para a cliente Sol, enquanto a correlação entre Regra 5 e CCR3 é maior para Sol do que para Dora.

No caso de Sol é interessante observar ainda que houve maior porcentagem de emissão de análise tanto por parte da cliente como por parte da terapeuta do que com a cliente Dora desde a Fase A.

Faz-se interessante ainda investigar mais detalhes a respeito das emissões de análises por terapeuta e cliente ${ }^{13}$. Sendo a Regra 5 a variável independente controlada na presente pesquisa, é razoável supor que um aumento nas emissões dessas respostas por parte da terapeuta gerou um aumento nas emissões de análises por parte das clientes, mas faz-se interessante uma análise mais minuciosa que indique se emissões de Regra 5 evocaram imediatamente emissões de CCR3 e O3 ou se essas últimas ocorreram em momentos posteriores na sessão terapêutica. Desse modo, foi realizada uma análise sequencial e calculadas as probabilidades condicionais das análises emitidas por ambas a fim de se investigar melhor essa questão. A partir dessa análise é possível calcular a probabilidade de um evento X ser seguido por um outro evento $\mathrm{Y}$. Tais análises serão apresentadas em seguida.

\section{B.1.2) Análise sequencial e probabilidade condicional de emissões de Regra 5 e análises das clientes}

No presente estudo, foi investigada, portanto, a probabilidade de emissões de Regra 5 da terapeuta serem seguidas por análises por parte das clientes. Tais investigações são apresentadas a partir da primeira fala da cliente depois de uma Regra 5 da terapeuta (Lag 1); bem como a segunda (Lag 3) e terceira (Lag 5) falas das clientes ${ }^{14}$.

\footnotetext{
${ }^{13}$ Ao categorizar sessões com o sistema FAPRS, usa-se um sistema de hierarquia para quando uma resposta possui propriedades de mais de uma categoria ao mesmo tempo, como mencionado anteriormente na seção de Método. Desse modo, há falas da terapeuta que possuem a função de Regra 5, mas também possuem função de Regra 3 (TRB1 e TRB2) ou Regra 2 e por isso não são contabilizadas como análise da terapeuta. Da mesma forma, categorias de análise das clientes que poderiam ser consideradas como CCR3 podem não ter sido reconhecidas como análise por serem também apresentações de CCR2, ou outras que poderiam ser consideradas como $\mathrm{O} 3$ não o foram por apresentarem também função de CCR1, por exemplo. Na tentativa de se verificar se tais respostas que ficaram "camufladas" poderiam fazer diferença nos dados apresentados, foi realizada uma segunda categorização, na qual essa segunda função de análise foi considerada sobre as demais, invertendo-se para elas a hierarquia. No entanto, a frequência de emissão dessas análises ao longo do experimento mostrou diferenças entre as fases experimentais semelhantes ao que já foi apresentado considerando-se a hierarquia na hora da categorização e por isso optou-se por não apresentar tais dados aqui.

${ }^{14}$ A sessão de follow-up não foi incluída nessa análise pela mesma ter sido categorizada posteriormente aos cálculos de análise sequencial. Além disso, na sessão de follow-up, houve apenas uma ocorrência de Regra 5 e nenhuma de CCR3 ou O3 no caso de Dora e no caso de Sol, houve apenas quatro ocorrências de Regra 5, uma de CCR3 e uma de $\mathrm{O} 3$.
} 
Na Tabela 10, observa-se que, durante a Fase A realizada com Dora, em cerca de $11 \%$ das vezes em que a terapeuta emitiu Regra 5, a cliente respondeu a ela com algum tipo de análise (O3 ou CCR3), tanto na primeira oportunidade de fala (Lag 1), como na segunda (Lag 3) e terceira (Lag 5). Na Fase B1, a cliente só respondeu com análises às emissões de Regra 5 da terapeuta em Lag 1 (também em cerca de 11\% das vezes); porém, observa-se que ocorreram apenas nove emissões de Regra 5 nessa fase (dez a menos do que na anterior) e dentre essas nove apenas uma foi respondida pela cliente com análise. Em seguida, observa-se que nas Fases BCs houve grande aumento na porcentagem de Regras 5 seguidas de análise das clientes (bem como a quantidade de análises emitidas), tanto em Lag 1, como nos demais, em relação às Fases Bs, sendo que na Fase B2 a cliente não emitiu nenhuma análise.

Tabela 10: Quantidade (n) e probabilidade (\%) de emissões de CCR3, O3 e a soma dos dois por Dora e Sol em resposta a uma Regra 5 da terapeuta, por fase experimental, em Lag 1, Lag 3 e Lag 5 ( $\mathrm{N}$ = quantidade de emissões de Regra 5).

\begin{tabular}{|c|c|c|c|c|c|c|c|c|c|c|c|c|c|c|c|}
\hline \multicolumn{16}{|c|}{ Dora } \\
\hline & \multicolumn{3}{|c|}{$\mathrm{A}(N=19)$} & \multicolumn{3}{|c|}{ B1 $(N=9)$} & \multicolumn{3}{|c|}{ BC1 $(N=75)$} & \multicolumn{3}{|c|}{ B2 $(N=4)$} & \multicolumn{3}{|c|}{$\operatorname{BC2}(N=42)$} \\
\hline & $\begin{array}{c}\text { Lag } \\
1\end{array}$ & $\begin{array}{c}\text { Lag } \\
3\end{array}$ & $\begin{array}{c}\text { Lag } \\
5\end{array}$ & $\begin{array}{c}\text { Lag } \\
1\end{array}$ & $\begin{array}{c}\text { Lag } \\
3\end{array}$ & $\begin{array}{c}\text { Lag } \\
5\end{array}$ & $\begin{array}{c}\text { Lag } \\
1\end{array}$ & $\begin{array}{c}\text { Lag } \\
3\end{array}$ & $\begin{array}{c}\text { Lag } \\
5\end{array}$ & $\begin{array}{c}\text { Lag } \\
1\end{array}$ & $\begin{array}{c}\text { Lag } \\
\mathbf{3}\end{array}$ & $\begin{array}{c}\text { Lag } \\
5\end{array}$ & $\begin{array}{c}\text { Lag } \\
1\end{array}$ & $\begin{array}{c}\text { Lag } \\
3\end{array}$ & $\begin{array}{c}\text { Lag } \\
5\end{array}$ \\
\hline$\%$ CCR3 & $0 \%$ & $0 \%$ & $0 \%$ & $11 \%$ & $0 \%$ & $0 \%$ & $3 \%$ & $0 \%$ & $0 \%$ & $0 \%$ & $0 \%$ & $0 \%$ & $24 \%$ & $19 \%$ & $21 \%$ \\
\hline$n$ CCR3 & 0 & 0 & 0 & 1 & 0 & 0 & 2 & 0 & 0 & 0 & 0 & 0 & 10 & 8 & 9 \\
\hline$\% \mathbf{O 3}$ & $11 \%$ & $11 \%$ & $11 \%$ & $0 \%$ & $0 \%$ & $0 \%$ & $23 \%$ & $13 \%$ & $7 \%$ & $0 \%$ & $0 \%$ & $0 \%$ & $12 \%$ & $10 \%$ & $5 \%$ \\
\hline$n \mathbf{O 3}$ & 2 & 2 & 2 & 0 & 0 & 0 & 17 & 10 & 5 & 0 & 0 & 0 & 5 & 4 & 2 \\
\hline $\begin{array}{c}\% \mathrm{CCR3}+ \\
\mathrm{O3}\end{array}$ & $11 \%$ & $11 \%$ & $11 \%$ & $11 \%$ & $0 \%$ & $0 \%$ & $25 \%$ & $13 \%$ & $7 \%$ & $0 \%$ & $0 \%$ & $0 \%$ & $36 \%$ & $29 \%$ & $26 \%$ \\
\hline $\begin{array}{c}n \mathrm{CCR3}+ \\
\mathrm{O3}\end{array}$ & 2 & 2 & 2 & 1 & 0 & 0 & 19 & 10 & 5 & 0 & 0 & 0 & 15 & 12 & 11 \\
\hline
\end{tabular}

\begin{tabular}{|c|c|c|c|c|c|c|c|c|c|c|c|c|c|c|c|}
\hline \multicolumn{16}{|c|}{ Sol } \\
\hline & \multicolumn{3}{|c|}{ A $(N=19)$} & \multicolumn{3}{|c|}{ B1 $(N=12)$} & \multicolumn{3}{|c|}{$\operatorname{BC1}(N=72)$} & \multicolumn{3}{|c|}{ B2 $(N=8)$} & \multicolumn{3}{|c|}{$\operatorname{BC2}(N=53)$} \\
\hline & $\begin{array}{c}\text { Lag } \\
1\end{array}$ & $\begin{array}{c}\text { Lag } \\
\mathbf{3}\end{array}$ & $\begin{array}{c}\text { Lag } \\
5\end{array}$ & $\begin{array}{c}\text { Lag } \\
1\end{array}$ & $\begin{array}{c}\text { Lag } \\
\mathbf{3}\end{array}$ & $\begin{array}{c}\text { Lag } \\
5\end{array}$ & $\begin{array}{c}\text { Lag } \\
1\end{array}$ & $\begin{array}{c}\text { Lag } \\
\mathbf{3}\end{array}$ & $\begin{array}{c}\text { Lag } \\
5\end{array}$ & $\begin{array}{c}\text { Lag } \\
1\end{array}$ & $\begin{array}{c}\text { Lag } \\
3\end{array}$ & $\begin{array}{c}\text { Lag } \\
5\end{array}$ & $\begin{array}{c}\text { Lag } \\
1\end{array}$ & $\begin{array}{c}\text { Lag } \\
\mathbf{3}\end{array}$ & $\begin{array}{c}\text { Lag } \\
5\end{array}$ \\
\hline$\%$ CCR3 & $0 \%$ & $0 \%$ & $0 \%$ & $0 \%$ & $0 \%$ & $0 \%$ & $17 \%$ & $10 \%$ & $7 \%$ & $0 \%$ & $0 \%$ & $0 \%$ & $13 \%$ & $8 \%$ & $6 \%$ \\
\hline$n$ CCR3 & 0 & 0 & 0 & 0 & 0 & 0 & 12 & 7 & 5 & 0 & 0 & 0 & 7 & 4 & 3 \\
\hline$\% 03$ & $26 \%$ & $11 \%$ & $0 \%$ & $58 \%$ & $17 \%$ & $17 \%$ & $21 \%$ & $17 \%$ & $18 \%$ & $63 \%$ & $38 \%$ & $13 \%$ & $40 \%$ & $30 \%$ & $28 \%$ \\
\hline$n \mathbf{O 3}$ & 5 & 2 & 0 & 7 & 2 & 2 & 15 & 12 & 13 & 5 & 3 & 1 & 21 & 16 & 15 \\
\hline $\begin{array}{c}\% \mathrm{CCR3}+ \\
\mathrm{O3}\end{array}$ & $26 \%$ & $11 \%$ & $0 \%$ & $58 \%$ & $17 \%$ & $17 \%$ & $38 \%$ & $26 \%$ & $25 \%$ & $63 \%$ & $38 \%$ & $13 \%$ & $53 \%$ & $38 \%$ & $34 \%$ \\
\hline $\begin{array}{c}n \mathrm{CCR3}+ \\
\mathrm{O3}\end{array}$ & 5 & 2 & 0 & 7 & 2 & 2 & 27 & 19 & 18 & 5 & 3 & 1 & 28 & 20 & 18 \\
\hline
\end{tabular}


Quanto à cliente Sol, foi observado que houve maior probabilidade de emissão de análises $(\mathrm{CCR} 3$ + O3) em resposta à Regra 5 (Lag 1) nas Fases Bs do que nas BCs e A. No entanto, ao observar mais minuciosamente esse dado, nota-se que na realidade $\mathrm{O} 3$ teve maior probabilidade de seguir Regra 5 nas Fases Bs, mas CCR3 teve uma maior probabilidade de ser emitida pós Regra 5 nas Fases BCs e $0 \%$ nas Fases A e B, tanto em Lag 1 como nos demais.

Na Tabela 11 pode-se observar a sequência contrária da interação. São aqui apresentados os dados referentes a porcentagem e quantidade de vezes que a terapeuta emitiu Regra 5 em resposta a análises emitidas pelas clientes (CCR3 + O3 no caso de Dora e CCR3 no caso de Sol), analisando-se a forma como a terapeuta respondeu a essas respostas das clientes. Observase que, em Lag 1, a terapeuta respondeu às análises de Dora com uma probabilidade semelhante nas Fases B1, BC1 e BC2, apesar da quantidade de análises feitas pela cliente e consequentemente pela terapeuta em resposta ser consideravelmente diferente em cada fase.

Tabela 11: Quantidade (n) e probabilidade (\%) de emissões de Regra 5 pela terapeuta em resposta a análises de Dora (CCR3+O3) e CCR3 de Sol, por fase experimental, em Lag 1, Lag 3 e Lag 5 ( $\mathrm{N}$ = quantidade de emissões de análise de Dora - CCR3 e O3 e quantidade de emissões de CCR3 de Sol).

\begin{tabular}{|c|c|c|c|c|c|c|c|c|c|c|c|c|c|c|c|}
\hline \multicolumn{16}{|c|}{ Dora } \\
\hline $\begin{array}{c}\mathrm{CCR3}+ \\
\mathrm{O3}\end{array}$ & \multicolumn{3}{|c|}{$\mathrm{A}(N=15)$} & \multicolumn{3}{|c|}{ B1 $(N=3)$} & \multicolumn{3}{|c|}{$\operatorname{BC1}(N=28)$} & \multicolumn{3}{|c|}{ B2 $(N=0)$} & \multicolumn{3}{|c|}{ BC2 $(N=29)$} \\
\hline & $\begin{array}{c}\text { Lag } \\
\mathbf{1}\end{array}$ & $\begin{array}{c}\text { Lag } \\
\mathbf{3}\end{array}$ & $\begin{array}{c}\text { Lag } \\
5\end{array}$ & $\begin{array}{c}\text { Lag } \\
1\end{array}$ & $\begin{array}{c}\text { Lag } \\
\mathbf{3}\end{array}$ & $\begin{array}{c}\text { Lag } \\
5\end{array}$ & $\begin{array}{c}\text { Lag } \\
1\end{array}$ & $\begin{array}{c}\text { Lag } \\
\mathbf{3}\end{array}$ & $\begin{array}{c}\text { Lag } \\
5\end{array}$ & $\begin{array}{c}\text { Lag } \\
1\end{array}$ & $\begin{array}{c}\text { Lag } \\
\mathbf{3}\end{array}$ & $\begin{array}{c}\text { Lag } \\
5\end{array}$ & $\begin{array}{c}\text { Lag } \\
1\end{array}$ & $\begin{array}{c}\text { Lag } \\
\mathbf{3}\end{array}$ & $\begin{array}{c}\text { Lag } \\
5\end{array}$ \\
\hline $\begin{array}{c}\% \text { Regra } \\
5\end{array}$ & $20 \%$ & $7 \%$ & $0 \%$ & $67 \%$ & $0 \%$ & $0 \%$ & $68 \%$ & $36 \%$ & $21 \%$ & - & - & - & $69 \%$ & $59 \%$ & $31 \%$ \\
\hline $\begin{array}{c}n \text { Regra } \\
5\end{array}$ & 3 & 1 & 0 & 2 & 0 & 0 & 19 & 10 & 6 & - & - & - & 20 & 17 & 9 \\
\hline \multicolumn{16}{|c|}{ Sol } \\
\hline CCR3 & \multicolumn{3}{|c|}{$\mathrm{A}(N=0)$} & \multicolumn{3}{|c|}{ B1 $(N=2)$} & \multicolumn{3}{|c|}{$\operatorname{BC1}(N=16)$} & \multicolumn{3}{|c|}{ B2 $(N=0)$} & \multicolumn{3}{|c|}{$\operatorname{BC2}(N=10)$} \\
\hline & $\begin{array}{c}\text { Lag } \\
1\end{array}$ & $\begin{array}{c}\text { Lag } \\
\mathbf{3}\end{array}$ & $\begin{array}{c}\text { Lag } \\
5\end{array}$ & $\begin{array}{c}\text { Lag } \\
1\end{array}$ & $\begin{array}{c}\text { Lag } \\
3\end{array}$ & $\begin{array}{c}\text { Lag } \\
5\end{array}$ & $\begin{array}{c}\text { Lag } \\
1\end{array}$ & $\begin{array}{c}\text { Lag } \\
3\end{array}$ & $\begin{array}{c}\text { Lag } \\
5\end{array}$ & $\begin{array}{c}\text { Lag } \\
1\end{array}$ & $\begin{array}{c}\text { Lag } \\
\mathbf{3}\end{array}$ & $\begin{array}{c}\text { Lag } \\
5\end{array}$ & $\begin{array}{c}\text { Lag } \\
1\end{array}$ & $\begin{array}{c}\text { Lag } \\
\mathbf{3}\end{array}$ & $\begin{array}{c}\text { Lag } \\
5\end{array}$ \\
\hline $\begin{array}{c}\% \text { Regra } \\
5\end{array}$ & - & - & - & $50 \%$ & $0 \%$ & $0 \%$ & $75 \%$ & $56 \%$ & $56 \%$ & - & - & - & $60 \%$ & $60 \%$ & $40 \%$ \\
\hline $\begin{array}{c}n \text { Regra } \\
5\end{array}$ & 0 & 0 & 0 & 1 & 0 & 0 & 12 & 9 & 9 & 0 & 0 & 0 & 6 & 6 & 4 \\
\hline
\end{tabular}

Já no caso de Sol, não foram observadas emissões de CCR3 nas Fases A e B2. Na Fase B1 apenas duas emissões ocorreram, sendo uma seguida de Regra 5. Porém, nas Fases BC2, o $N$ aumentou consideravelmente e a probabilidade de emissão de Regra 5 em seguida aos CCR3s também sofreu aumento (em número e porcentagem). 
O conjunto desses dados de Dora indicam uma probabilidade de análises emitidas pela cliente em resposta à Regra 5 maior nas Fases BCs do que nas Fases Bs, sendo que, ao menos em Lag 1, o mesmo não é observado na relação contrária (terapeuta respondendo à cliente). Porém, se a terapeuta respondeu com uma probabilidade similar às análises da cliente nessas diferentes fases experimentais, questiona-se a razão da cliente responder com análises com uma probabilidade maior nas Fases BCs do que nas Fases Bs. Em outras palavras, qual será a razão de ser mais provável que a cliente emita análises em respostas às emitidas pela terapeuta em uma ou outra fase, se a terapeuta responde a elas de forma semelhante? Terão sido as respostas em Lag 3 e 5 emitidas na Fase BCs a responsável pela maior emissão de análises da cliente nessas fases? Além disso, como observado anteriormente na Figura 6, há mais emissões de CCR3 por Dora durante as Fases BCs do que durante as demais fases, levando ao questionamento sobre a razão de, não apenas a quantidade, mas também a qualidade (CCR3 x O3) das respostas de análise da cliente serem diferentes nas Fases BC do que nas demais.

Questionamento semelhante pode ser levantado em relação à cliente Sol. O fato do número de emissões de CCR3 ser nulo nas Fases A e B2 e tão baixo na Fase B1 leva a dúvidas sobre a razão dele ter sofrido tamanho aumento nas Fases BCs. O que está sendo questionado é qual a razão das emissões de Regra 5 nas Fases Bs e A não terem sido seguidas nenhuma vez por CCR3 e o serem tantas vezes nas Fases BCs.

Supõe-se que pode ter havido algum evento de valor evocativo nas Fases BCs que tenha favorecido a emissão de análises (CCR3 e O3) pela cliente Dora e de CCR3 pela cliente Sol seguindo às da terapeuta. Duas hipóteses, não excludentes, puderam ser levantadas. A primeira delas é que o fato da terapeuta ter emitido mais análises de forma geral nas Fases BCs pode ter tido esse valor evocativo, aumentando a probabilidade de emissões de análise ou CCR3 em resposta à Regra 5 por parte das clientes.

A segunda hipótese diz respeito ao tipo ou qualidade das emissões de Regra 5 realizadas pela terapeuta em cada uma das fases experimentais. Talvez as análises que a terapeuta emitiu durante as Fases Bs e BCs sejam diferentes em alguma propriedade, relacionadas por exemplo a seu conteúdo, sendo aquelas emitidas nas Fases BCs mais evocativas em certo aspecto do que as outras. Uma microanálise das emissões de Regra 5 e das emissões de CCR3 e O3, apresentada a seguir, pode indicar algumas possibilidades de respostas e de caminhos para maiores investigações futuras. 
B.1.3) Microanálise dos comportamentos de analisar da terapeuta e das clientes: relações a partir de subcategorizações

Conforme apresentado na seção de Método foram criadas subcategorias para os comportamentos de análise da terapeuta (Regra 5) e das clientes (CCR3 e O3) a fim de se obter um conhecimento mais preciso sobre tais análises ao longo do delineamento experimental ${ }^{15}$.

O tipo de análises feito em cada uma das fases experimentais não foi uma variável controlada ao longo dessa pesquisa, pois seriam muitas variáveis a serem manipuladas, levando a problemas na condução da pesquisa e na posterior análise dos dados, mas é interessante fazer uma análise descritiva da função e conteúdo das análises que foram realizadas durante o delineamento. É importante ressaltar que a microanálise desses dados foi realizada com um baixo número de respostas. Como mencionado acima, por ser necessário manter a emissão das demais regras da FAP, durante todo o delineamento experimental posterior à Fase A, poucas emissões de Regra 5 ocorreram mesmo nas Fases BCs, ao menos comparando com emissões da Regra 3. Com isso, a análise abaixo foi realizada baseada nessas poucas respostas (tanto a Regra 5, como análises emitidas pelas clientes), mas por apresentarem dados interessantes, serão aqui relatadas.

Sendo assim, as subcategorias referentes ao comportamento da terapeuta podem ser agrupadas em parâmetros de função e conteúdo; enquanto aquelas referentes ao comportamento das clientes foram agrupadas apenas pelo seu conteúdo.

Desse modo, as subcategorias criadas para o comportamento do terapeuta podem apresentar as funções de modelação/dar exemplo de análise ( $\mathrm{T}$ analisa); evocação do comportamento de análise do cliente (T evoca) e consequenciação da emissão de análise por parte do cliente (T consequencia). Em relação ao conteúdo, podem ser divididas em análises do comportamento do cliente fora de sessão (T O3), dentro da sessão (T C3) ou paralelo entre dentro e fora de sessão (T paralelo); ou ainda em relação a comportamentos problema (T A1) ou de melhora (T A2).

As subcategorias criadas para o comportamento dos clientes podem ser divididas da mesma forma que as do terapeuta em relação ao conteúdo: sobre o comportamento do cliente fora de sessão (C O3), dentro de sessão (C C3) ou o paralelo entre eles (C paralelo) ${ }^{16}$; ou em relação a comportamentos problema (C A1) ou de melhora (C A2).

\footnotetext{
${ }^{15}$ A sessão de follow-up não foi incluída nessa análise, pelos mesmos motivos apresentados acima a respeito da análise sequencial.

${ }^{16}$ As respostas denominadas aqui como $C 3$ e $C$ Paralelo são subdivisões da resposta chamada $C C R 3$ nas demais seções do texto.
} 


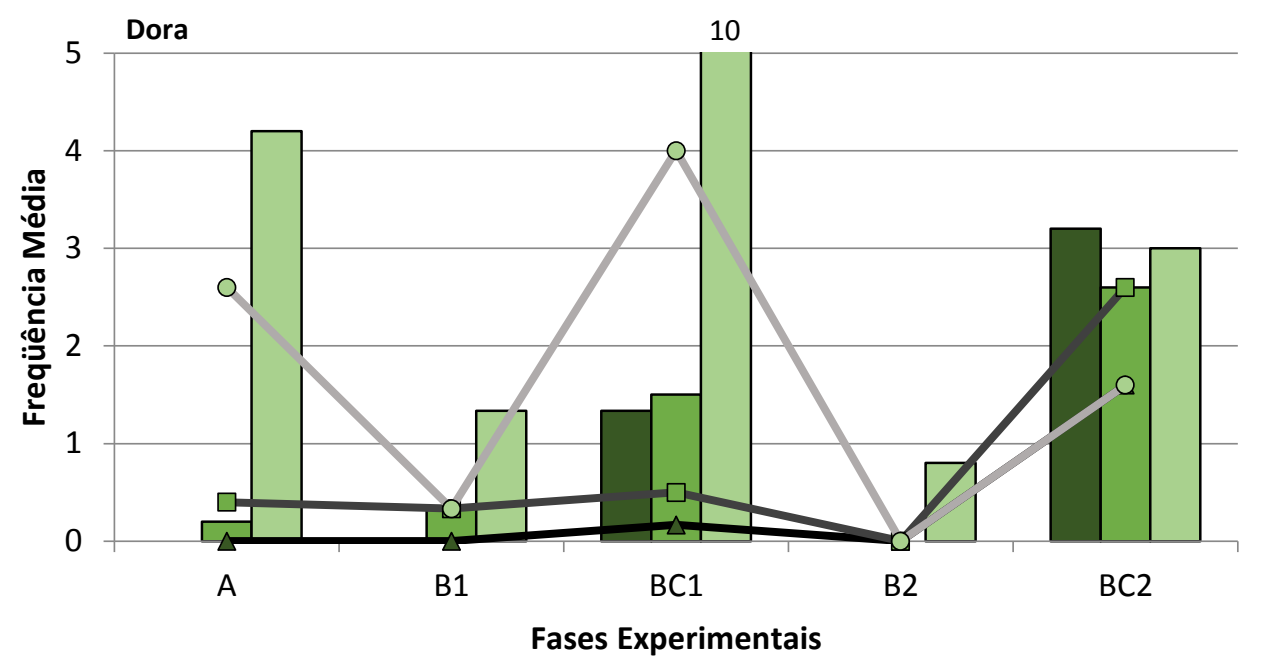

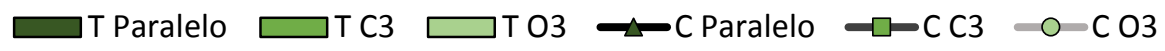

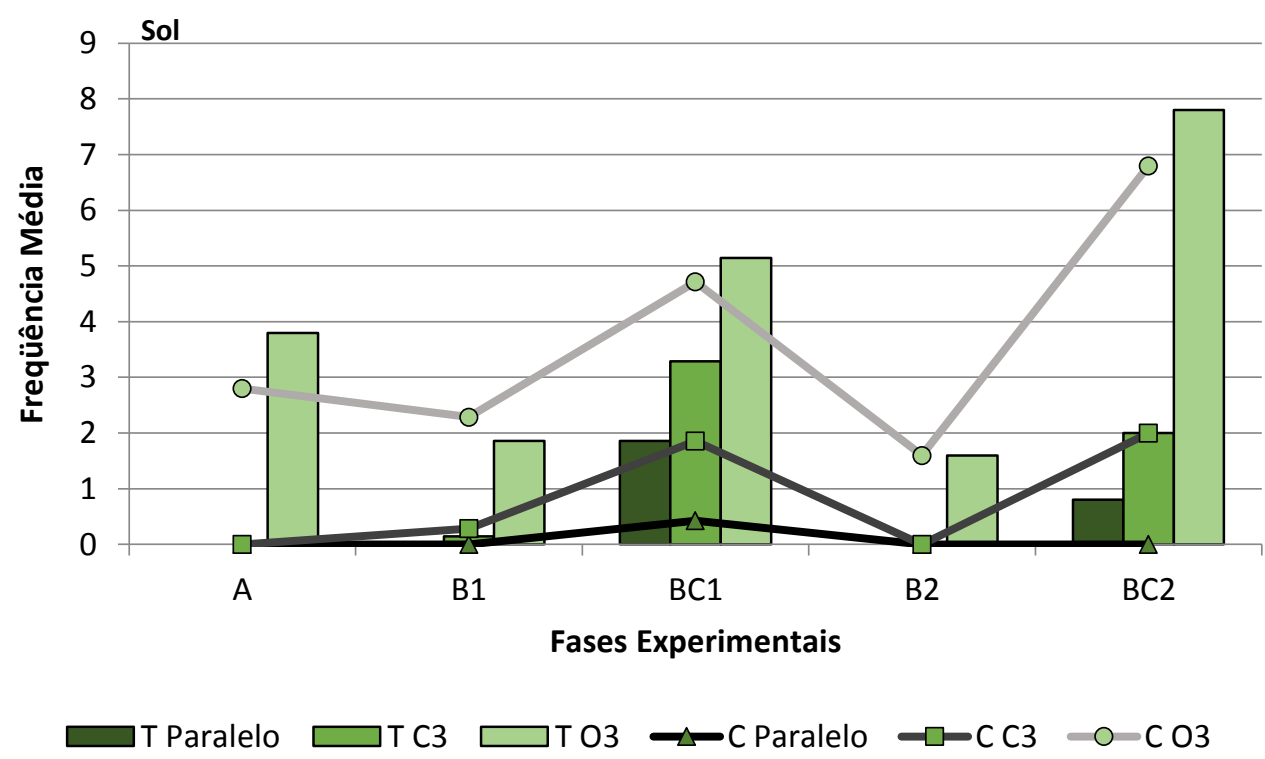

Figura 7: Frequência média de emissão das subcategorias referentes ao conteúdo das análises realizadas pela terapeuta ( $\mathrm{T}$ - Regra 5 - barras) e pelos clientes (C - CCR3 e O3 linhas), divididas em paralelo, C3 (dentro de sessão) e O3 (fora de sessão), emitida em cada fase experimental, para as clientes Dora e Sol. As escalas dos gráficos são individualizadas para cada participante.

Na Figura 7, são apresentadas para cada fase experimental as frequências médias por sessão de emissão de respostas de análise emitidas pela terapeuta e pelas clientes, separadas em análises de dentro da sessão, fora da sessão e seus paralelos. Ao final da seção anterior, foi questionada a razão de haver mais análises por parte de Dora e mais CCR3 por parte de Sol em sequência às emissões de Regra 5 da terapeuta durante as Fases BCs e emissões dessas serem quase nulas durante as demais fases. Observa-se na Figura 7, que o conteúdo das análises da própria terapeuta mostra-se diferente nas Fases BCs quando comparado com as demais fases. 
Em geral, foram mais frequentes emissões de análise sobre o comportamento fora de sessão das clientes (O3) do que as demais análises, com exceção da Fase BC2 com a cliente Dora. Quanto às análises das clientes, o mesmo é observado. Foram mais frequentes emissões de $\mathrm{O} 3$ do que as demais, com exceção da mesma Fase BC2 da cliente Dora e da Fase B1 com a mesma cliente.

Além disso, observam-se mais emissões de análises sobre o comportamento de dentro da sessão (T C3) por parte da terapeuta nas Fases BCs do que nas demais, o que se assemelha à emissão dessas análises (C C3) por parte das clientes. Observa-se também que a terapeuta realizou paralelos entre análises de dentro e fora da sessão apenas nas Fases BCs, com ambas as clientes, ao mesmo tempo em que poucas emissões de paralelos são observadas por parte das clientes ao longo do delineamento, mas apenas nas Fases BCs. Sendo assim, possivelmente pode-se atribuir a maior emissão das análises das clientes sobre o que ocorre dentro da terapia, nas Fases BCs, ao conteúdo das análises emitidas pela própria terapeuta.

Tais dados correspondem ao que era esperado, considerando-se o delineamento proposto. Isto é, dificilmente seria possível conduzir as sessões experimentais das Fases A e Bs sem a emissão total de análises, porém, as que foram emitidas foram prioritariamente sobre os comportamentos das clientes fora de sessão. Mesmo essas análises, sobre comportamentos de fora da sessão, tem sido consideradas como Regra 5 na literatura da FAP e tradicionalmente como CCR3 quando emitidas pelo cliente (Kohlenberg \& Tsai, 1991; Tsai et al., 2009). Porém tem sido enfatizado em alguns trabalhos de forma mais ou menos direta a importância das análises em sessões FAP envolverem conteúdo de dentro da sessão e seus paralelos com fora de sessão (Abreu et al., 2012; Abreu-Rodrigues \& Sanabio-Heck, 2005; Kohlenberg \& Tsai, 1991; Tsai et al., 2009; Villas-Bôas, Meyer, Kanter, \& Callaghan, 2015). Desse modo, tem sido entendido que análises sobre os comportamentos de dentro da sessão e de paralelos de dentro para fora são análises mais típicas de sessões FAP por analisarem a própria relação terapêutica e o processo de modelagem que foi realizado em sessão, relacionando-o com situações externas à terapia com o objetivo de promover a transferência do aprendizado que ocorreu em sessão para fora da sessão. Assim, era esperado que as análises referentes à Regra 5 realizadas em um processo de terapia FAP fossem focadas nas melhoras que ocorreram na sessão e na possibilidade de que melhoras semelhantes ocorressem fora de sessão.

A Figura 8, de fato, mostra que a maioria das análises realizadas pela terapeuta na Fase BC2 com a cliente Dora e nas Fases BCs com a cliente Sol foram sobre melhora, a única exceção se dá na Fase BC1 com a cliente Dora. 

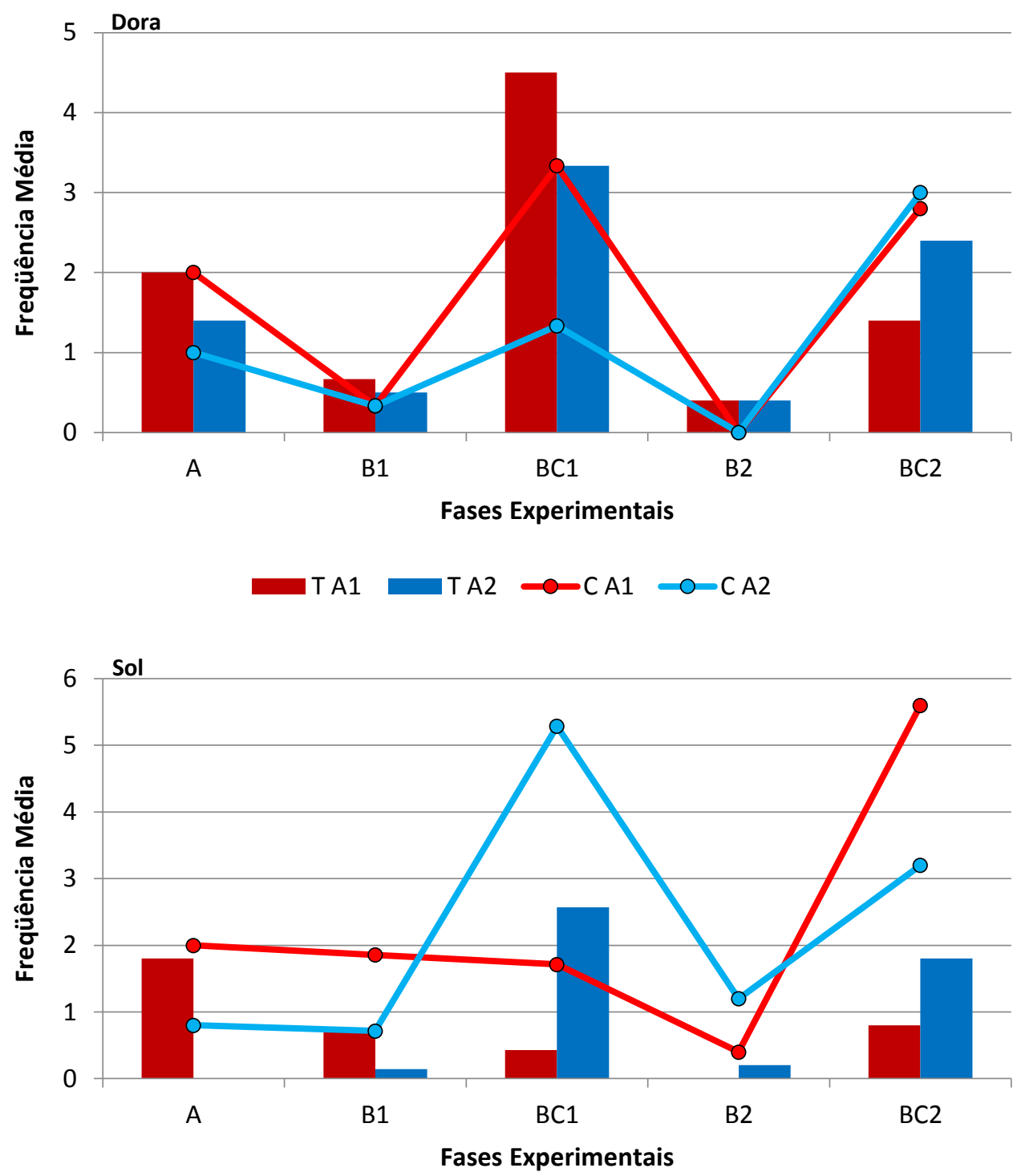

$\longrightarrow$ TA1 $\quad$ TA2 $\longrightarrow$ CA1 $\longrightarrow$ CA2

Figura 8: Frequência média de emissão das subcategorias referentes ao conteúdo das análises realizadas pela terapeuta ( $\mathrm{T}$ - Regra 5 - barras) e pelos clientes $(\mathrm{C}$ - CCR3 e O3 linhas), divididas em análises sobre comportamento problema (A1) ou de melhora (A2), emitida em cada fase experimental, para as clientes Dora e Sol. As escalas dos gráficos são individualizadas para cada participante.

No entanto, uma análise mais detalhada mostra que as análises externas (O3) na Fase $\mathrm{BC} 1$ de Dora, foram prioritariamente sobre problemas (ocorridas com uma alta frequência concentrada na Sessão 6), mas as análises internas (CCR3) e os paralelos foram prioritariamente sobre melhoras, o que corresponde ao que é esperado que ocorra com as análises realizadas em um processo FAP, como mencionado acima (tal dado pode ser observado no Apêndice I, em uma tabela apresentando a frequência por sessão de todas as subcategorias realizadas). 
Por sua vez, observa-se que as variações no conteúdo das análises emitidas pelas clientes são similares às da terapeuta, indicando a ocorrência de um diálogo, com sintonia sobre o que era analisado por terapeuta e cliente. As únicas exceções ocorrem na Fase BC2, com a cliente Sol (a cliente Dora apresentou o mesmo número de análises de problemas e de melhoras na Fase B1 e nenhuma análise na Fase B2). No caso da cliente Sol, já foi previamente observado, na Figura 4, grande variação nas emissões de CCRs nas duas últimas fases experimentais, e discutiu-se brevemente que tais oscilações ocorreram possivelmente em função de uma mudança no foco das análises realizadas em relação às fases anteriores (mudança de foco para questões com peso corporal). Acredita-se que o dado apresentado na Fase BC2, na Figura 8, é mais um desses indícios.

Por fim, faz-se interessante analisar a função das respostas correspondentes à Regra 5. Tradicionalmente a Regra 5 da FAP foi descrita como sendo o comportamento de analisar o responder do cliente pelo terapeuta (Kohlenberg \& Tsai, 1991; Tsai et al., 2009). Mas em especial em textos mais recentes tem sido descrita a importância da Regra 5 ser vista de forma mais ampla e não apenas como uma forma de descrever o comportamento do cliente, mas sim como uma forma de ensinar o cliente a descrever seu próprio comportamento (Abreu et al., 2012; Villas-Bôas et al., 2015). Ou seja, nesse processo, exemplos de análise devem ser dados, mas também seria importante evocar a emissão de análises e consequenciar adequadamente as tentativas do cliente de emiti-las. Assim, como discutido em Villas-Bôas et al. (2015) outros autores da análise do comportamento indicam a importância de análises serem emitidas pelo próprio cliente como uma forma de aumentar o contato desses com as contingências relevantes de seu ambiente. Nesse sentido, o comportamento da terapeuta pode ser analisado em relação à função que apresentou (dar modelo, evocar ou consequenciar análises dos clientes) nas diferentes fases experimentais.

Observa-se na Figura 9 que, com a cliente Dora, o comportamento de analisar o responder da cliente foi mais frequente do que evocar e consequenciar análises da própria cliente em todas as fases experimentais. Porém, emissões de Regra 5 com a função de evocar ou consequenciar análises emitidas por Dora foram mais emitidas nas Fases BCs do que nas demais fases experimentais, indicando que nas Fases BCs houve uma maior preocupação em levar a cliente a analisar seu próprio comportamento do que nas demais fases. 


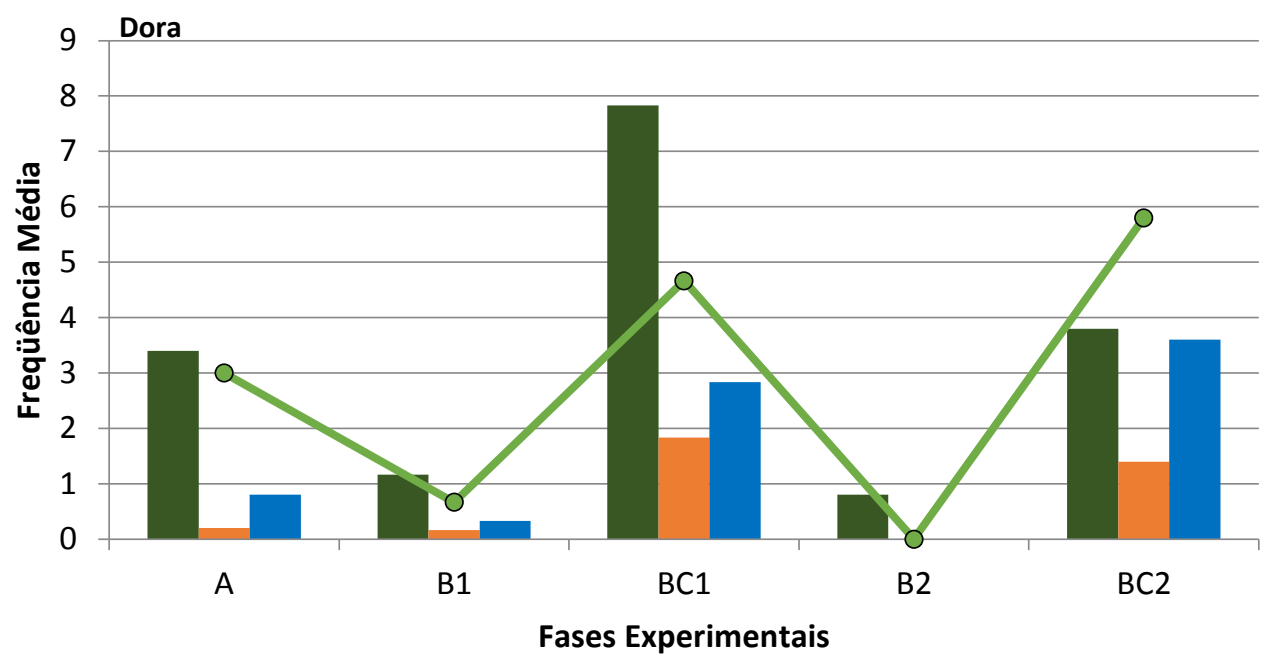

T analisa $\quad$ T evoca T consequencia $-\mathrm{C}$ analisa (CCR3 + O3)

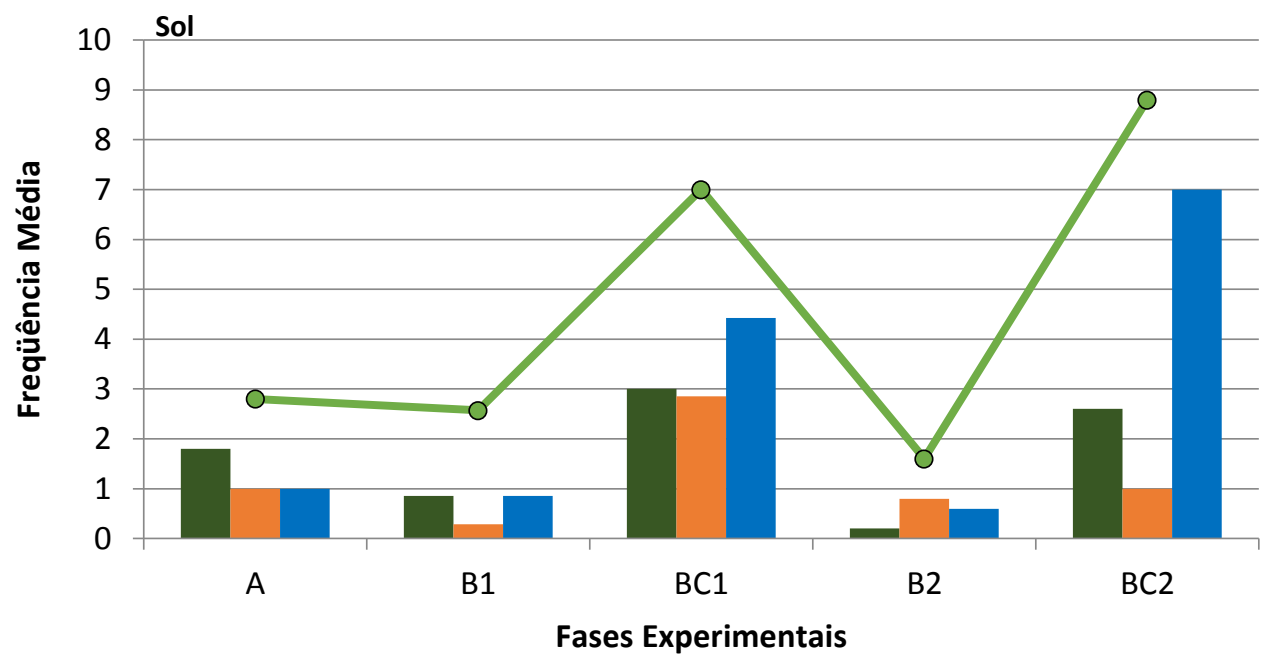

T analisa $\quad$ T evoca T consequencia $-\mathrm{C}$ analisa $(\mathrm{CCR} 3+\mathrm{O} 3)$

Figura 9: Frequência média de emissão das subcategorias referentes à função das análises realizadas pela terapeuta (Regra 5 - barras), divididas em terapeuta (T) analisa, evoca ou consequencia análises realizadas pela cliente e frequência de emissão das análises realizadas pelas clientes Dora e Sol (C - CCR3 e O3 - linha), em cada fase experimental.

Com a cliente Sol, emitir análises (T analisa) foi a subcategoria mais frequente dentre essas três formas de Regra 5 apenas na Fase A e ainda assim com uma frequência pouco mais alta do que as demais subcategorias. Nas Fases BCs há mais emissões de análises da terapeuta e de consequenciação das análises do que nas demais fases do experimento, e na Fase BC1 o mesmo ocorre com evocações de análises. É curioso observar que nas Fases BCs, a subcategoria com mais frequência de emissão é a consequenciação o que possivelmente é resultado uma maior frequência de emissões de análise por parte da cliente do que nas demais fases. Como observado anteriormente, de fato, a cliente Sol emitiu mais análises do que a 
cliente Dora e o fez com uma correlação em relação à Regra 5 maior do que com a primeira cliente. O conjunto desses dados indica uma maior facilidade de analisar (ou de evocar e modelar tal repertório) para a cliente Sol do que para a cliente Dora.

Os dados propiciados pela análise das subcategorias indicam que, nas Fases BCs, houve uma maior preocupação do que nas demais fases em auxiliar as clientes a analisarem seus próprios comportamentos, focando mais em melhoras que ocorreram em sessão e os paralelos com o cotidiano das clientes que possibilitam melhoras em seu dia a dia, ou seja, focando-se no objetivo de transferir os progressos em sessão para fora (generalizar, como descrito pela bibliografia da FAP). O fato de se ter mantido esse foco como objetivo, possivelmente foi responsável pela maior emissão de análises por parte das clientes durante essas fases, e por haver mais análises sobre o que ocorre em sessão (CCR3) por parte delas. Possivelmente emitir tais análises sobre o aqui e agora teve a princípio um caráter aversivo, e dificilmente ocorreria naturalmente no repertório das clientes, sem uma ajuda ativa da terapeuta.

Microanálises adicionais sobre parte desses dados foram realizadas por Mendes (2014) ${ }^{17}$. A autora conduziu uma investigação detalhada das análises emitidas pela cliente Sol e terapeuta durante a Fase BC1, através de uma análise sequencial das subcategorias já aqui mencionadas e da categorização dessas mesmas falas através do SMCCIT (Zamignani, 2007), a fim de identificar detalhes nas emissões de análise por parte da terapeuta e da cliente Sol em sessão. Diferentemente da análise sequencial apresentada acima, no trabalho de Mendes (2014), a sequência entre análises de terapeuta e cliente não foi considerada em Lag 3 ou 5 . As análises emitidas por terapeuta e cliente foram consideradas em sequência, uma após a outra, mesmo que tivessem falas entre elas que não tenham sido categorizadas como análises (CCR1 ou CCR2, por exemplo).

A análise sequencial das subcategorias indicou que cerca de metade dos comportamentos de análise emitidos pela cliente na Fase BC1 foram antecedidos por Regra 5 mesmo que em momentos bem anteriores na sessão. Além disso, quando a Regra 5 emitida era do tipo evocação, a maioria de suas emissões foram seguidas por análises da cliente (83,3\% quando as análises evocadas eram referentes a comportamentos em sessão, 66,6\% quando referentes a paralelos e $100 \%$ quando referentes a comportamento extra sessão). Quando a Regra 5 era referente a consequenciação da terapeuta às análises da cliente, em cerca de metade das vezes foi seguida por análises da cliente $(54,5 \%$ referente a análises intrassesão; 52,9\% referente a

\footnotetext{
${ }^{17}$ A então aluna de graduação Ester B. Mendes realizou uma pesquisa em um programa de Iniciação Científica da Universidade de São Paulo, sob orientação da Profa. Dra. Sonia Meyer e co-orientação de Alessandra VillasBôas detalhando parte dos dados do presente trabalho e complementando-os.
} 
análises extrassesão; e apenas $25 \%$ referente a paralelos). Por fim, análises emitidas pela própria terapeuta foi o que menos foi seguido de análises por parte da cliente (20\% referente a análises intrassesão; 26,7\% referente a análises extrassesão; e 66,7\% referente a paralelos).

Além disso, as análises emitidas por terapeuta e cliente foram categorizadas também a partir do SMCCIT. Observa-se que nas sessões da cliente Sol da Fase BC1, a categoria do SMCCIT mais emitida de forma geral, dentre as análises realizas pela cliente foi a de Estabelecimento de relações entre eventos (50 ocorrências), seguida pela categoria de Relato (23 ocorrências) e Melhora (9 ocorrências). No caso da terapeuta, dentre as análises emitidas, a maioria são consideradas como sendo de Interpretação (40 ocorrências), seguida pela categoria de Aprovação (33 ocorrências) e Solicitação de Reflexão (19 ocorrências).

A autora também apresentou um cruzamento entre as subcategorias baseadas no sistema FAPRS e as categorias identificadas pelo SMCITT e concluiu que dentre as análises emitidas pela terapeuta categorizadas como Terapeuta emite análise (e não evoca ou consequencia), a maioria foi referente a Interpretações, pelo sistema SMCCIT. Quanto às analises referentes a consequenciação das análises feitas pela cliente, a maioria tratou-se também de Interpretações (quando as análises foram referentes a análises extra sessão e paralelos) ou Aprovação (quando referente a análises de dentro da sessão). Porém, ao evocar análises da cliente (a forma encontrada de produzir mais análises por parte de Sol) a maioria consistiu em Solicitação de Reflexão.

No conjunto dos dados, portanto, a autora concluiu que evocações de análises por meio de solicitações de reflexão foi a forma mais efetiva de evocar análises no comportamento da cliente.

Sendo assim, supõe-se que o aumento de evocações e consequenciações para as análises de Sol realizadas na Fase BC1 (Figura 9), em relação a fase anterior, foi possivelmente a grande responsável pelo o aumento de análises intrassessão (Figura 7) realizado pela cliente.

\section{B.2) Relação entre análises e os CCR1 e CCR2}

A seguir serão apresentadas suscintamente investigações sobre um possível impacto que a Regra 5 e análises emitidas pelas clientes poderiam ter sobre os CCR1s e CCR2s emitidos. Tal investigação faz-se interessante na medida em que foram realizadas análises sobre o comportamento das clientes em sessão durante alguns momentos do processo terapêutico (Fases BCs), como visto anteriormente, e cabe o questionamento se, o fato do comportamento em sessão ter sido analisado influencia suas próximas emissões. 
Em uma inspeção visual da Figura 10 não são observadas influências evidentes das análises emitidas tanto por terapeuta como pelas clientes sobre as porcentagens de emissão de CCR2 de nenhuma das duas clientes.
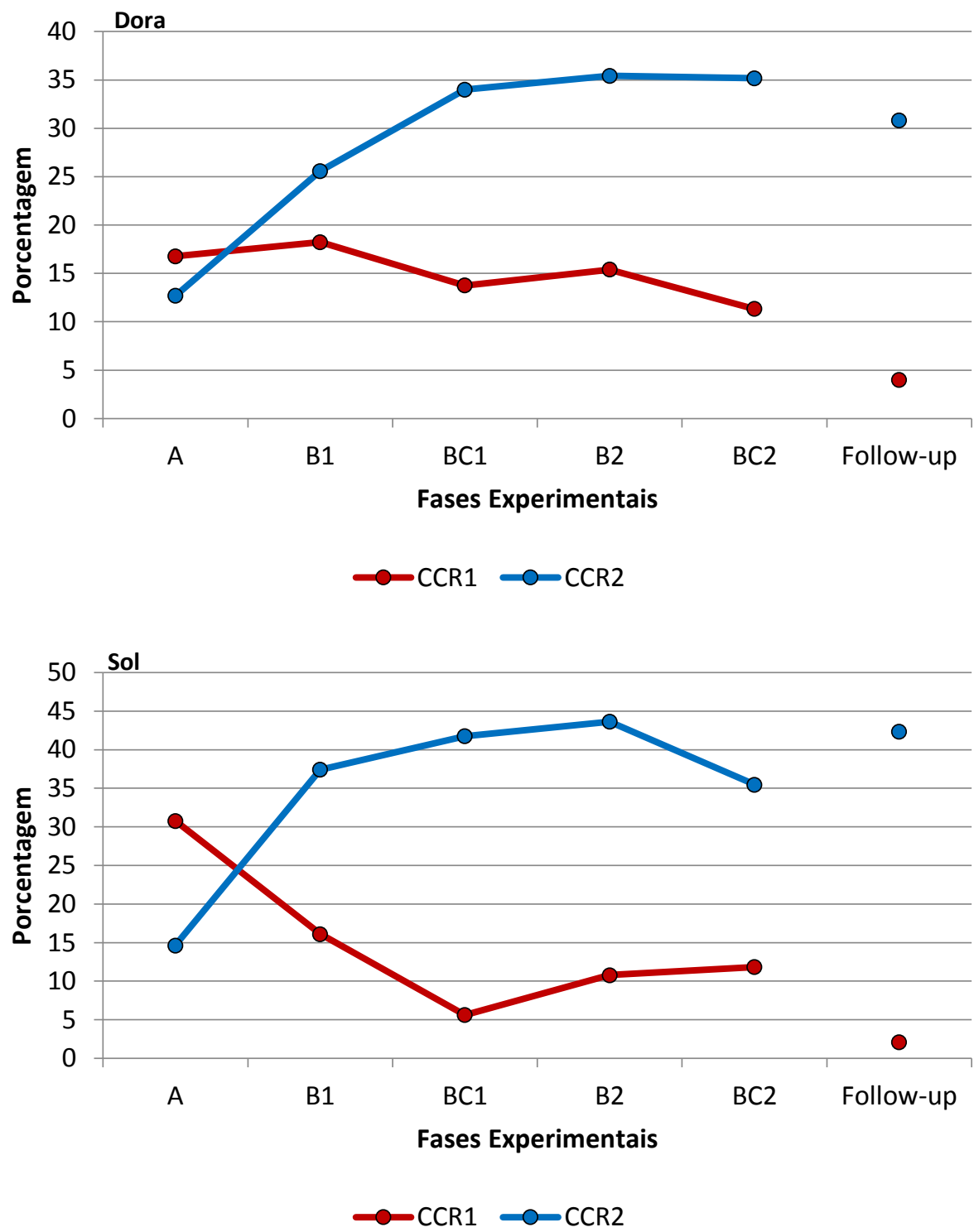

Figura 10: Porcentagem de emissão das categorias referentes à CCR1 e CCR2, dentre todas as respostas emitidas pelas clientes Dora e Sol, por fase experimental e sessão de followup. As escalas dos gráficos são individualizadas para cada participante.

Porém, para a cliente Dora, quando observada a porcentagem de emissão de CCR1 em cada fase experimental, verifica-se que na Fase BC1 há uma queda nas emissões de CCR1 em relação a porcentagem emitida na Fase B1 (de 18,23\% para 13,75\%). Em seguida, na Fase B2, a porcentagem volta a subir levemente para 15,39\%, voltando a cair para $11,33 \%$ na Fase BC2. Tal dado sugere que, de forma geral, talvez possa ter havido uma pequena influência das 
emissões de análise sobre o comportamento problema de Dora em sessão. Ao analisarmos esse mesmo dado sessão a sessão, como na Figura 4, tal possível influência não é observada, especialmente devido às variações nas porcentagens de CCRs ocorridos de uma sessão a outra.

No caso de Sol, também é observado uma diminuição de CCR1 na Fase BC1, em relação a porcentagem emitida na Fase B1 (de 16,10\% para 5,62\%). Em seguida, um aumento ocorre na Fase B2, chegando a 10,78\% de emissões de CCR1. Porém, diferentemente de Dora, no caso de Sol há um novo aumento, ainda que pequeno, para 11,83\% na última fase.

Pelos dados apresentados e discutidos anteriormente, não se pode atribuir a diminuição de CCR1 na Fase BC1 apenas à emissão de análises para nenhuma das clientes, afinal a consequenciação dos CCRs (Regra 3 - TRB1 e TRB2) parece ter tido grande influência nos dados.

Sendo assim, o conjunto dos dados apresentados, não se constituem como evidências fortes e/ou consistentes de uma possível influência de respostas de análises sobre os CCR1s e CCR2s de nenhuma das duas clientes. Por outro lado, muito se tem a dizer a respeito da influência que emissões de Regra 5 geraram no comportamento de analisar das clientes.

Em suma, para a cliente Dora ocorreu um aumento de análises em geral (CCR3 e O3) nas Fases BC, enquanto as maiores diferenças para a cliente Sol ocorreram em relação a análises sobre o que acontece na sessão terapêutica (CCR3). Ainda, observa-se que o conteúdo das análises da terapeuta e clientes mantiveram-se semelhantes ao longo do delineamento, de modo que, quando a terapeuta passou a emitir mais análises sobre o que ocorreu dentro da sessão, as clientes o passaram a fazer também; terapeuta e cliente mantiveram em geral o mesmo foco entre melhora versus comportamento problema na maior parte das sessões, sendo em sua maioria melhoras predominantes nas Fases BCs. Além disso, nas Fases BC parece ter ocorrido uma maior preocupação da terapeuta em evocar e modelar o repertório de análise nas clientes, de modo que não focou-se apenas em apresentar análises prontas, o que possivelmente gerou o aumento de análises emitidas por elas. Isso é complementado pelos dados de Mendes (2014) que indicam que, ao menos na Fase BC1 da cliente Sol, foi mais provável a emissão de análises por parte da cliente frente a evocações da terapeuta que solicitassem reflexões da cliente.

Maiores discussões sobre as influências das análises da terapeuta sobre os comportamentos das clientes serão realizadas adiante (Seção E), em conjunto com os próximos dados apresentados. 


\section{C) Dados e análises sobre comportamento extra sessão}

Serão apresentados a seguir os dados referentes aos comportamentos das clientes fora da sessão terapêutica. Como mencionado anteriormente na seção de Método, várias formas de coleta de dados foram utilizadas com cada cliente e seus resultados serão detalhados a seguir. Diferentemente das seções anteriores, nessa seção todos os dados serão apresentados primeiramente para a cliente Dora e em seguida, todos os dados da cliente Sol. Está sendo considerado mais produtivo obter um conjunto dos dados externos de cada cliente separadamente, já que aparentemente eles se complementam nas evidências de possíveis melhoras ou pioras de cada uma. Ao final da seção será feito um apanhado geral sobre os dados externos de ambas as clientes bem como um aprofundamento de discussões sobre essa forma de registro.

\section{C.1) Cliente Dora}

\section{C.1.1) Registro externo}

Ao longo do delineamento experimental, a cliente Dora registrou a frequência de seu próprio comportamento, concentrado em algumas respostas definidas em sessão em conjunto com a terapeuta.

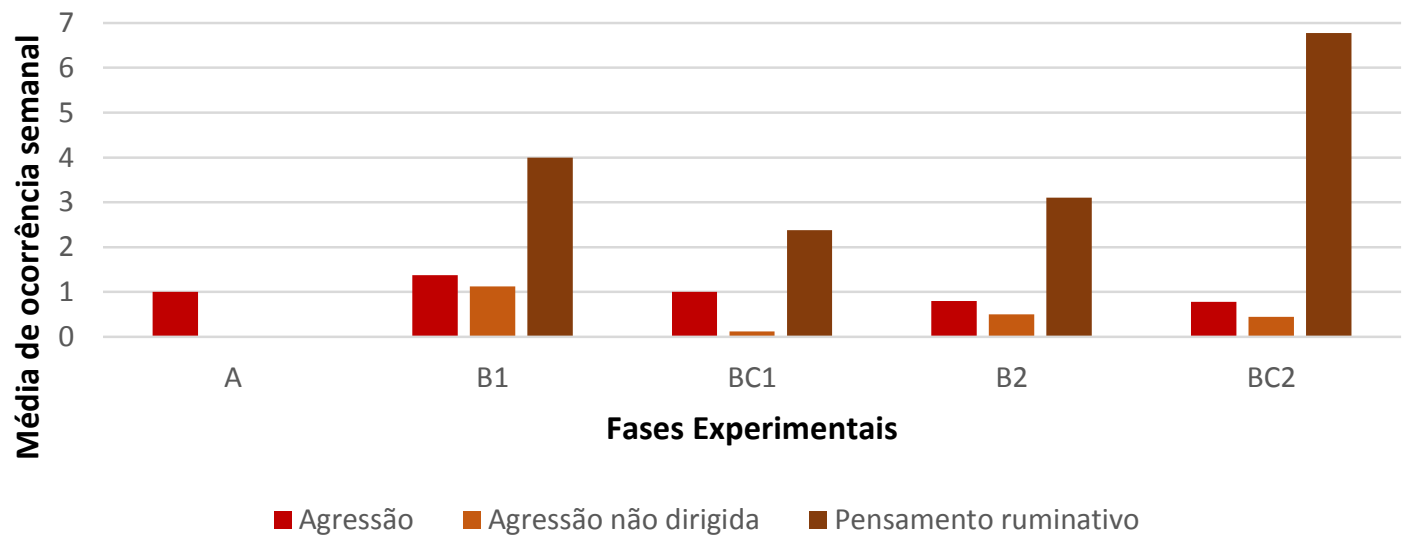

Figura 11: Média semanal da frequência de emissão das respostas registradas pela cliente Dora, por fase experimental (a resposta Pensamentos Ruminativos passou a ser registradas apenas na Fase B1).

Na Figura 11 é possível observar a média das respostas registradas pela cliente Dora em cada fase experimental, sendo que todas elas são consideradas como comportamentos problema de Dora. A frequência de cada uma das três respostas foi variável ao longo das sessões experimentais, mas em média é possível observar que a cliente registrou, por exemplo, uma 
resposta de Agressão por semana, durante a Fase A. A média de frequência de registro dessa resposta sofreu um leve aumento ao ser introduzida a FAP, na Fase B1, e diminuiu sutilmente, porém de forma constante, ao longo das fases experimentais. Já respostas denominadas como Agressão não dirigida, não ocorreram na Fase A e passaram a ocorrer na Fase B1, apresentando uma média de registros menor, porém oscilante, ao longo das fases experimentais subsequentes. Por fim, os Pensamentos ruminativos, que ainda não estavam na folha de registro na Fase A, foram registrados com uma média de frequência maior do que as demais respostas a partir da Fase B1 sofrendo oscilação, mas apresentando uma média de frequência maior ao final do delineamento do que nas fases anteriores.

Tais dados indicam uma melhora, bastante modesta, em relação às respostas de Agressão e Agressão não dirigida e uma piora em relação às repostas de Pensamento ruminativo da cliente; porém, vale apontar que o impacto desses pensamentos sobre pessoas a seu redor e sobre ela mesma deve ser menor do que as o das agressões dirigidas ou não. No entanto, o fato do dado não ser expressivo e devido à forma como o registro foi realizado (ao início de cada sessão e com tamanha aversividade para a cliente, conforme relatado no Método), leva a suspeitar da confiabilidade do mesmo.

\section{C.1.2) Observador externo}

Na Figura 12 é possível observar a pontuação dada pelo observador externo a cada uma das respostas no final de cada fase experimental. Infelizmente a terapeuta não recebeu os dados coletados da Fase B2.

Mesmo na ausência desses dados, é possível observar, de forma geral, uma redução na pontuação dada aos comportamentos problema e um aumento na pontuação dada aos comportamentos de melhora ao longo do delineamento. Mais especificamente, observa-se que a resposta de Agressão obteve pontuação 3 nas Fases A e B1, caindo para 2 nas Fases BCs. A resposta de Esquiva do conflito, sofreu uma queda na pontuação nas três primeiras fases e um aumento final na última, mas mantendo-se abaixo de sua pontuação inicial. Por sua vez, Agressão não dirigida manteve-se constante nas três primeiras fases, com uma pontuação 4, caindo para 2 na última fase. 

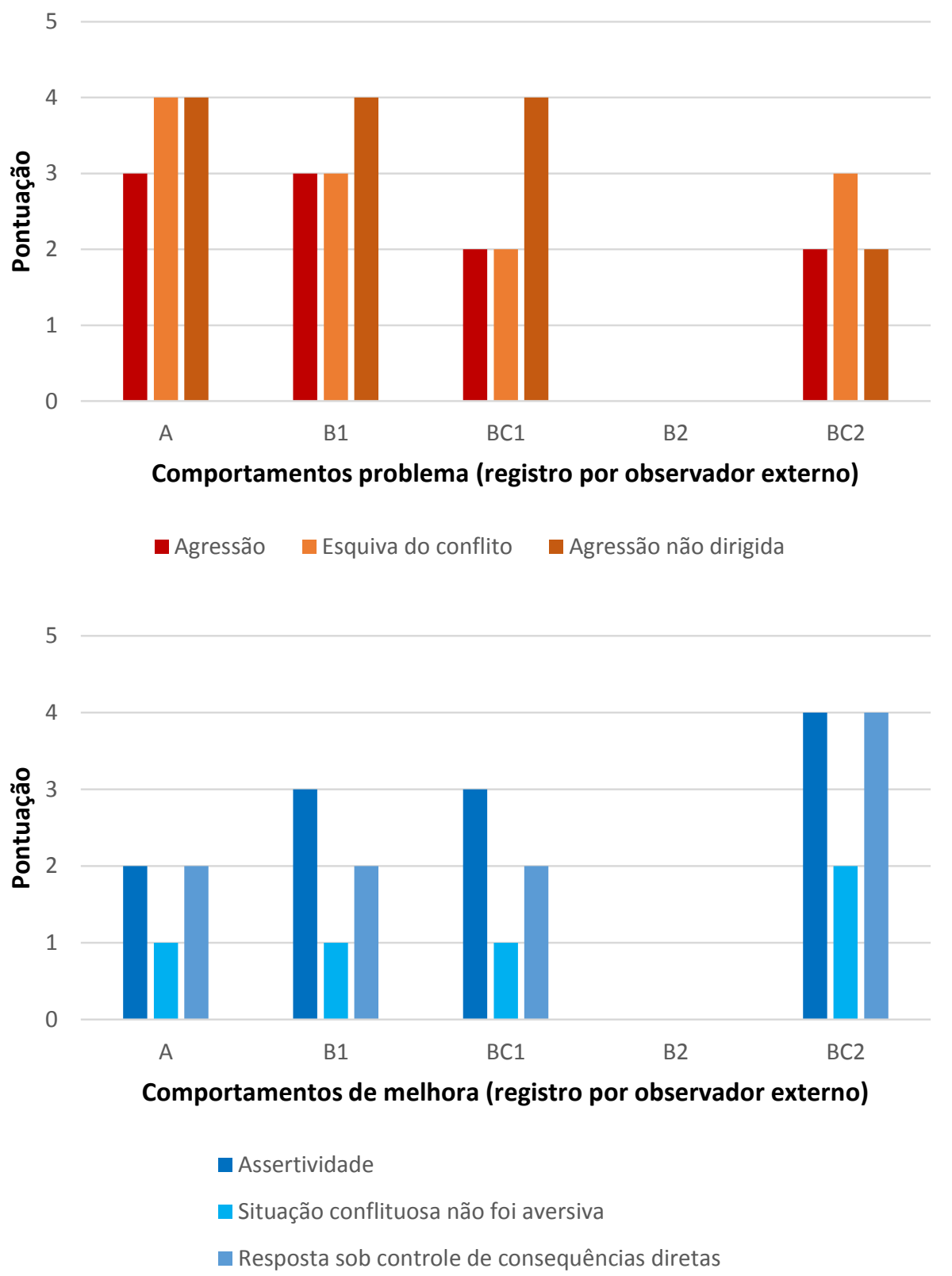

Figura 12: Média da pontuação dada a cada uma das respostas registradas em uma escala de 5 pontos (Raramente - 1; Poucas vezes - 2; Algumas vezes - 3; Muitas vezes - 4; Com frequência - 5), pelo observador externo, por fase experimental.

Por sua vez, a resposta Assertividade, foi pontuada inicialmente com 2, subindo para 3 nas Fases B1 e BC1, chegando a pontuação 4 na Fase BC2. Já as respostas que indicam que a Situação conflituosa não foi aversiva e Respostas sob controle de consequências diretas de suas ações mantiveram-se constantes nas três primeiras fases e sofreram um aumento na última, sendo que a segunda chegou a atingir a pontuação 4 .

Devido à falta do registro da Fase B2, não é possível identificar se as melhoras de Dora estiveram relacionadas com algumas das fases experimentais, mas observa-se que houve uma 
melhora nos comportamentos de Dora (aumento de comportamentos de melhora e diminuição dos comportamentos problema) segundo o registro do observador externo.

\section{C.1.3) $E A S-40$}

Uma vez por mês foi aplicado o EAS-40, no meio e final de cada fase experimental. Os dados coletados através desse instrumento foram tabulados e estão sendo apresentados na Figura 13.

Observa-se que a cliente Dora apresentou escores bastante próximos da média da população para a maioria das dimensões analisadas, durante quase todo o delineamento experimental. Apenas a dimensão Ansiedade, encontrou-se bastante abaixo da média da população em todas as aplicações do instrumento.

Dentre os itens agrupados pela escala, aquele nomeado como psicoticismo é o que mais corresponde às principais queixas da cliente Dora, seguido por obsessão compulsão. Em específico nessas dimensões é curioso notar a variação apresentada por Dora ao longo das fases experimentais. É verificado que, depois de um mês que foi introduzida a FAP, ponto 1 da Fase B1, ocorreu uma diminuição no escore desses itens, em relação ao que foi apresentado na Fase A, aproximando-os da média da população. O escore de Psicoticismo manteve-se constante ao longo dessa fase, enquanto que o de Obsessão compulsão sofreu um leve aumento. Na Fase $\mathrm{BC} 1$, ambas as dimensões sofreram uma diminuição, em seus escores, evidenciada no final da fase para Psicoticismo e já na primeira aplicação do instrumento na fase (depois de um mês em vigência) para Obsessão compulsão. Durante a Fase B2, ocorreu aumento nos escores de ambas as dimensões a partir da segunda aplicação da escala, ou seja, depois de dois meses que a VD foi retirada, continuando a subir até o final da fase. Finalmente na Fase BC2, psicoticismo manteve-se constante na primeira aplicação em relação à última aplicação da fase anterior, mas diminuiu seu escore na segunda aplicação. Para a dimensão de obsessão compulsão, observase um grande aumento inicial, na primeira aplicação da escala em relação a aplicação anterior, mas uma posterior diminuição, ainda ao longo da fase. Faz-se interessante observar que tais dados talvez indiquem uma influência da VD (Regra 5) sobre o comportamento da cliente em relação a essas dimensões, que como dito, são as que se aproximam mais das queixas trabalhadas na terapia de Dora. Parece haver um efeito ainda que atrasado sobre essas respostas, que tiveram seus escores diminuídos ao longo das fases em que as análises foram apresentadas. 

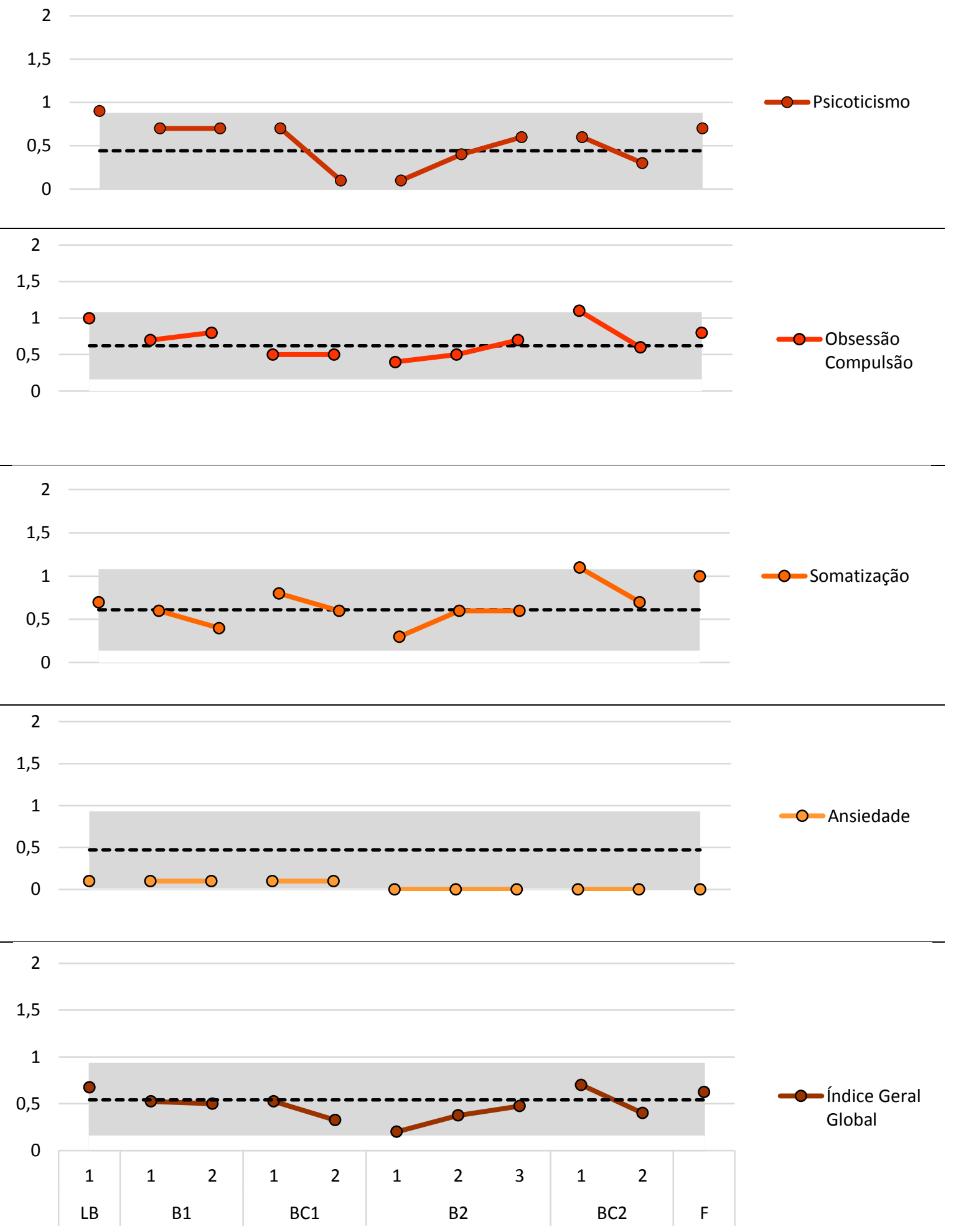

Figura 13: Escores obtidos para cada uma das dimensões medidas pela Escala de Avaliação dos Sintomas (Laloni, 2001) e o índice geral global da cliente Dora. A escala foi aplicada 11 vezes e a figura indica o momento do delineamento experimental no qual a aplicação foi realizada. A linha tracejada em cada quadro indica a média da população para o sexo feminino em cada uma das dimensões e a área em cinza, o intervalo de desvio padrão. F corresponde a follow-up. 


\section{C.1.4) 01 e $\mathrm{O} 2$}

Os dados sobre o comportamento externos da cliente apresentados até o momento indicam de forma geral uma diminuição dos comportamentos problema da cliente e um aumento dos comportamentos de melhora, ainda que de forma bastante sutil, na maioria dos casos. Uma análise realizada a partir da emissão de Os em sessão tem o objetivo de complementar tais análises. Assim, foi analisada a porcentagem de $\mathrm{O} 1$ e de $\mathrm{O} 2$ emitida em cada fase experimental (e na sessão de follow-up), dentre todas as respostas emitidas pela cliente naquela fase.

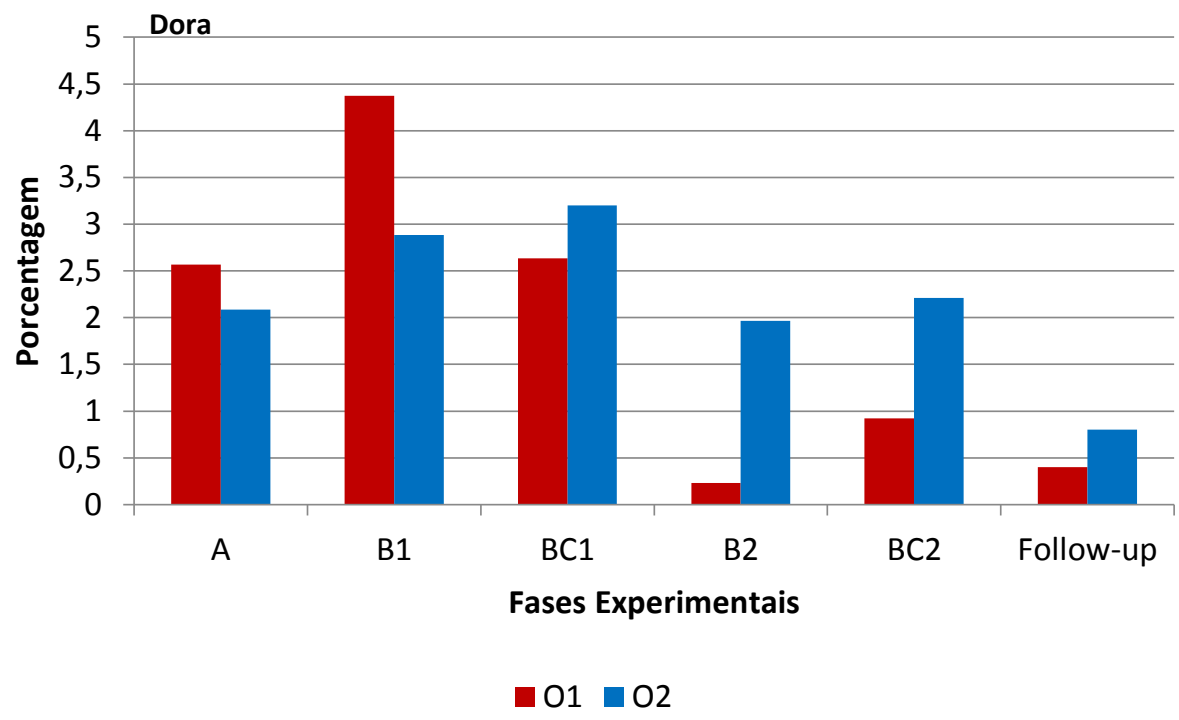

Figura 14: Porcentagem de emissão das categorias referentes a $\mathrm{O} 1$ e $\mathrm{O} 2$, dentre todas as respostas emitidas pela cliente Dora, por fase experimental e sessão de follow-up.

Observa-se, na Figura 14, que a cliente Dora falava mais sobre comportamentos problema do que de melhora durante a Fase A. A porcentagem de emissão de ambos os comportamentos subiu quando foi introduzida a FAP na Fase B1, sendo que o aumento ocorrido com o comportamento problema (O1) foi maior (de 2,57\% para 4,37\%) do que aquele ocorrido com os comportamentos de melhora ( $\mathrm{O} 2$ - de 2,09\% para 2,88\%). Na Fase BC1 houve um aumento de $\mathrm{O} 2$ em relação a fase anterior, enquanto $\mathrm{O} 1$ começou a diminuir em porcentagem. Nas fases subsequentes, a porcentagem de emissão de ambos os comportamentos caiu, sendo que $\mathrm{O} 1$ permaneceu com menor porcentagem de emissão do que O2, apesar de ter sofrido um aumento da Fase B2 para BC2. Nota-se que, ao longo das fases experimentais, a diferença entre a porcentagem de emissão de $\mathrm{O} 1$ e $\mathrm{O} 2$ aumentou. Além disso, observa-se uma diminuição na soma das porcentagens de $\mathrm{O} 1$ e $\mathrm{O} 2$ nas duas últimas fases e na sessão de followup em comparação com as primeiras fases, indicando que falou-se menos sobre ambos nessas 
últimas fases. Na sessão de follow-up, a diferença entre $\mathrm{O} 1$ e $\mathrm{O} 2$ diminuiu em relação à última fase experimental, mas $\mathrm{O} 1$ continuou sendo emitido com uma porcentagem menor do que $\mathrm{O} 2$. A partir desses dados, não é observado nenhum efeito aparente com a introdução da VD que se mostre sistemático nas Fases BCs.

\section{C.1.5) Episódios de Os}

Na Figura 15, está sendo apresentada a frequência média de respostas que correspondem a comportamento problema e comportamento de melhora que foram detectados pelas falas da cliente Dora, ocorridas na semana seguinte à sessão experimental, tal como detalhado e justificado na seção de Método. Tal dado está sendo nomeado como Episódios de O1, referente ao comportamento problema e Episódios de O2, referentes a comportamentos de melhora.

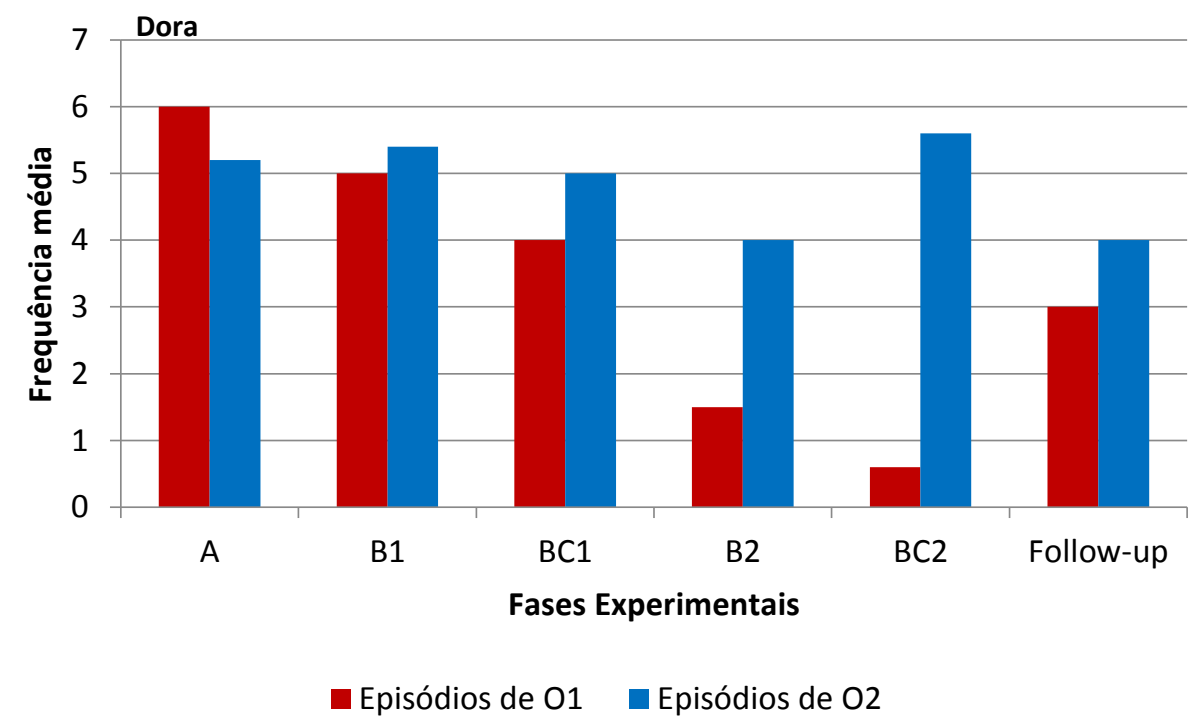

Figura 15: Frequência média de emissão de Episódios de $\mathrm{O} 1$ e Episódios de $\mathrm{O} 2$ detectados nas falas da cliente Dora, por fase experimental e sessão de follow-up.

Observa-se então, na Figura 15, que foram relatados mais Episódios de O1 do que Episódios de $\mathrm{O} 2$ nas semanas que ocorreram posteriormente a sessões da Fase A. Na Fase B1, ao ser introduzida a FAP, esse número caiu para Episódios de O1, enquanto os Episódios de O2 aumentaram levemente. Nas Fases BC1 e B2 há uma visível diminuição na frequência de emissão de ambas as respostas, sendo que a diminuição de Episódios de O1 é consideravelmente maior (de cinco ocorrências, na Fase B1 para 1,5, na Fase B2) do que as de Episódios de $\mathrm{O} 2$ (de 5,4 ocorrências, na Fase B1 para 4, na Fase B2). Finalmente, na Fase BC2, houve aumento na frequência de ambas as respostas, quando comparadas à fase anterior, sendo que tal aumento foi consideravelmente maior para os Episódios de O2 (de quatro ocorrências 
na Fase B2 para 5,6 na Fase BC2) do que para os Episódios de O1 (de 1,5 ocorrências na Fase $\mathrm{B} 2$ para 1,8 na Fase BC2). Ao comparar o dado dessa última fase experimental com o dado apresentado na primeira, Fase A, observa-se que ao longo do período experimental da terapia mais Episódios de $\mathrm{O} 2$ passaram a ser mencionados após as sessões experimentais enquanto Episódios de $\mathrm{O} 1$ diminuíram em frequência. Além disso, assim como observado na Figura 14, nota-se que a diferença entre a frequência de Episódios de $\mathrm{O} 1$ e a de $\mathrm{O} 2$ também aumentou ao longo do delineamento. Ainda, da mesma forma como observado na figura anterior, na sessão de follow-up, a diferença entre Episódios de $\mathrm{O} 1$ e de $\mathrm{O} 2$ diminuiu em relação à última fase experimental, mas Episódios de O1 continuaram a ser emitidos com uma porcentagem menor do que de Episódios de $\mathrm{O} 2$.

\section{C.1.6) Relatos e impressões clínicas}

Os dados apresentados pelo instrumento de coleta do observador externo (Figura 12), pela análise dos Os (Figura 14) e dos Episódios de Os (Figura 15), apesar de não serem expressivos, corroboram as impressões clínicas da terapeuta e da cliente sobre o desenvolvimento terapêutico de Dora. Como comentado acima, os registros realizados pela própria cliente também indicam certa melhora clínica, em especial quanto às respostas de Agressão, mas com evidências extremamente modestas.

Durante o delineamento experimental, a terapeuta tinha dúvidas a respeito da confiabilidade do registro que estava sendo realizado pela cliente por este estar sendo preenchido ao início das sessões e pela evidente e expressa aversividade que este apresentava a ela. Ao final do experimento, de fato os registros realizados pouco refletiram as percepções clínicas dos progressos realizados em sessão, apesar de não se mostrarem contrários a tais percepções.

Algumas sessões após o término da coleta de dados os registros realizados foram discutidos em sessão entre terapeuta e cliente. Na ocasião, a terapeuta contou à cliente que os registros não evidenciavam os progressos percebidos e discutidos por ambas durante o processo e foi conversado sobre a razão disso. A cliente contou que sente que melhorou muito com a terapia e naquele momento sentia muita falta da terapia em momentos sem sessão. Segue abaixo parte da conversa que se seguiu sobre a dificuldade com o registro:

C: Eu tô vivendo um processo muito particular de restauro, vamo lembrar da questão do restauro. Então se, de repente, não apareceu (a melhora no registro), pode até ser que não apareceu, mas você sabe que mudou, eu sei também que mudou muito. (...) 
Aquele registro de certa forma era difícil até pra mim. Que tinha momentos que eu não sabia avaliar o registro.

T: (...) a sensação que eu tenho é essa. Que muitas vezes o que você tava registrando era confuso, eram principalmente as explosões, né? E eu me lembro de um dia que você chegou falando que você tinha tido uma explosão e aí era uma situação com o seu filho que você tinha ficado muito, muito brava com ele. Não lembro o que tinha acontecido, mas você tinha falado com ele e você foi mega assertiva na forma como você falou com ele, mas a emoção que veio foi de explosão. Você conseguiu segurar e lidar com ele de uma forma super legal, mas é confuso né? O que que eu tô anotando? É o tamanho da raiva que eu sinto ou é o tamanho do grito que eu dou? Acho que isso é que é difícil. É disso que você tá falando quando você fala "eu não sei avaliar"?

$C:$ É, é, é... porque às vezes eu tinha que trazer pra você avaliar a situação pra eu tentar ser mais verdadeira porque no começo era mais fácil, mas depois eu me dei conta que assim era mais fácil, mas significa assim que eu fiz correto?

T: No começo era mais fácil porque de fato acho que tudo era explosão e aí deixou de ser...

C: Então, mas eu acho que assim, não deixou de ser a explosão, só que a explosão passou a ser analisada muitas vezes por uma questão assertiva. Porque assertividade na minha vida é uma coisa que não existia.

Percebe-se pelo diálogo acima, em especial pela última fala da cliente, que ainda nesse momento há certa confusão da cliente sobre o que considerar como explosões (agressão) e a principal diferença disso para respostas assertivas, com certa dificuldade ainda de discriminar entre o sentimento de raiva que acompanha tais situações e a forma de lidar com a situação, podendo ser assertiva ou agressiva.

Como já mencionado anteriormente, Korotitsch e Nelson-Gray (1999), apontam que o comportamento de auto registro envolve a discriminação de sua ocorrência, além da própria produção do registro em si, sendo que as duas respostas são importantes para que o registro seja preciso. Apesar dos esforços aqui realizados, parece que Dora encontrou dificuldades em ambos os repertórios.

Tal dificuldade de discriminação pode realmente ter levado a certa dificuldade em registrar seu comportamento, mas tanto terapeuta como cliente (assim como o observador externo) descrevem mudança na forma de reagir de Dora a situações de fortes emoções, apresentando diminuição na agressividade ao mesmo tempo em que seu comportamento assertivo aumentou. Além disso, as impressões clínicas tanto da terapeuta como da cliente apontam para um aumento de enfrentamento de situações difíceis, com melhores resultados 
atingidos, aumento do círculo social e aumento de preocupações e atitudes voltadas para seu bem-estar. De forma geral, acredita-se que houve melhora no dia a dia de Dora mesmo que as evidências experimentais não sejam fortes.

Resta ainda saber se tal melhora está relacionada com emissões da Regra 5, ocorridas nas Fases BC ou se foi efeito da condução da terapia FAP, através da modelagem de repertório ocorrido em sessão. Antes dessa análise ser realizada, os dados do comportamento externo da segunda cliente, Sol, serão apresentados e analisados.

\section{C.2) Cliente Sol}

\section{C.2.1) Registro externo}

Na Figura 16, estão sendo apresentados os registros realizados por Sol ao longo de todo delineamento. Após ter sido encerrado o delineamento experimental, Sol optou continuar registrando seus comportamentos por acreditar que tal registro lhe ajudava, como mencionado acima.

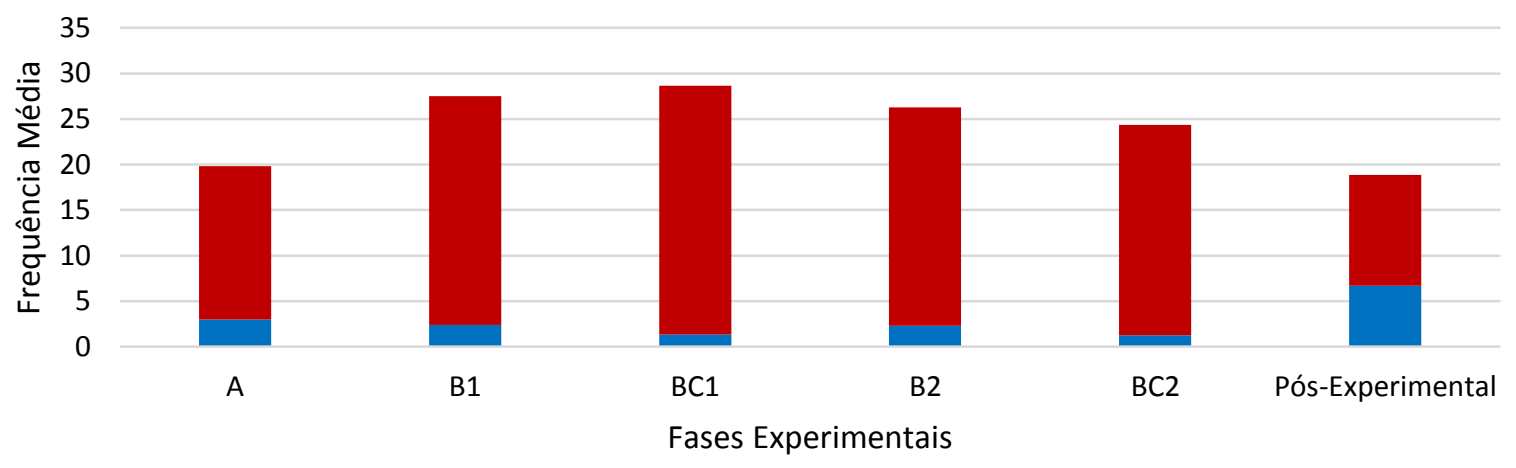

- Respostas reforçadas positivamente (melhora)

- Respostas reforçadas negativamente (problema)

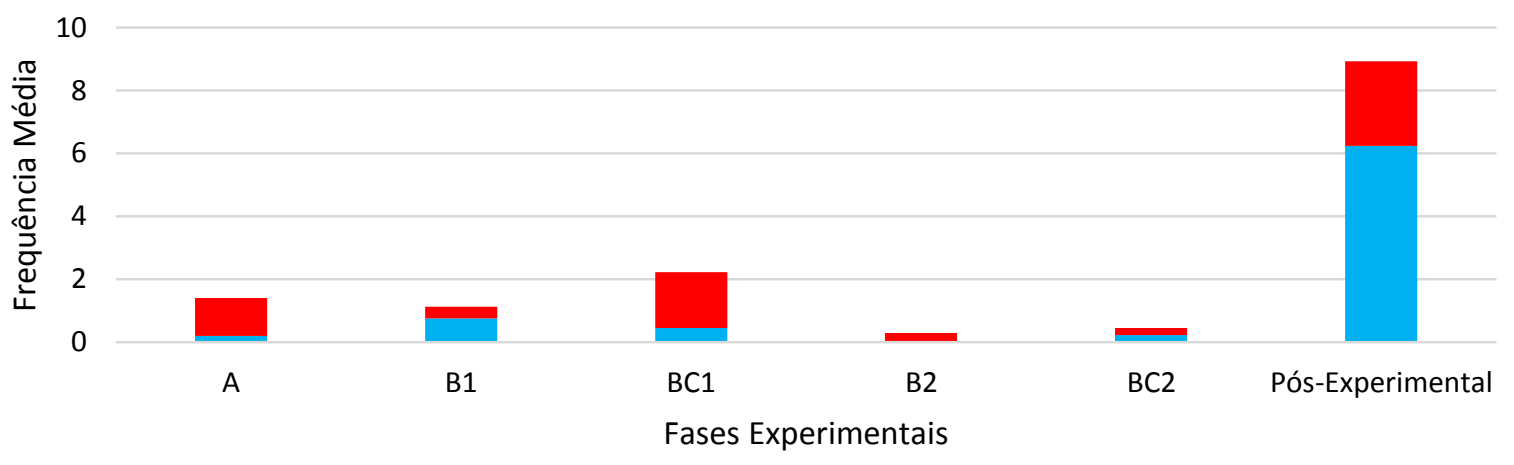

- Privou-se de contato com reforçadores positivos sociais

- Entrou em contato com reforçadores positivos sociais

Figura 16: Média da frequência de emissão das respostas registradas semanalmente pela cliente Sol, por fase experimental. 
Como pode ser observado, não ocorreram grandes mudanças no registro de comportamento de melhora ou de comportamento problema durante todo o período experimental. No entanto, depois de encerrado o delineamento, é possível observar diminuição na frequência de emissão dos comportamentos problema e um considerável aumento na frequência de emissão dos comportamentos de melhora.

Faz-se interessante observar que durante o delineamento experimental foi pouco frequente a cliente Sol ter vontade de entrar em contato com amigos (contato com reforçadores sociais), e na maioria dos poucos momentos em que o teve, acabou não fazendo-o. Depois de finalizado o delineamento, passou a ser mais frequente Sol ter vontade de procurar por amigos (o que por si só já é considerada uma melhora para ela) e passou a fazê-lo com bastante frequência. Quanto aos comportamentos de fazer coisas pelos outros em detrimento de suas próprias vontades, observa-se também uma melhora posterior ao final do experimento, porém mais modesta do que a dos comportamentos relatados acima.

\section{C.2.2) $E A S-40$}

Assim como feito para a cliente Dora, os dados coletados com a cliente Sol através do instrumento EAS-40 foram tabulados e são apresentados na Figura 17. Dentre as dimensões descritas pela escala, não há nenhuma que parece corresponder às principais queixas de Sol. Porém nota-se que Sol apresentou escores altos no início da pesquisa na maioria das dimensões, sendo a única exceção a de Somatização. Ao longo do delineamento experimental observou-se uma diminuição nos escores aproximando-os da média da população em quase todas as dimensões da escala. A única exceção foi Obsessão compulsão, na qual houve diminuição nos escores apresentados, mas permanecendo acima da média da população.

Sendo assim, os dados apresentados pelo EAS-40 indicam que Sol apresentava índices acima da média da população para Psicoticismo, Obsessão compulsão e Ansiedade, sendo que estes foram diminuindo ao longo do delineamento, mas sem diferenças entre as fases experimentais, ao contrário de Dora. Além disso, faz-se curioso observar que, com exceção da dimensão de Psicoticismo, em todas as demais dimensões, há um aumento do escore na primeira aplicação realizada em cada fase experimental. Dado semelhante foi apresentado na Figura 4, na qual observou-se um aumento de CCR1 e uma diminuição de CCR2 a cada mudança de fase experimental. 

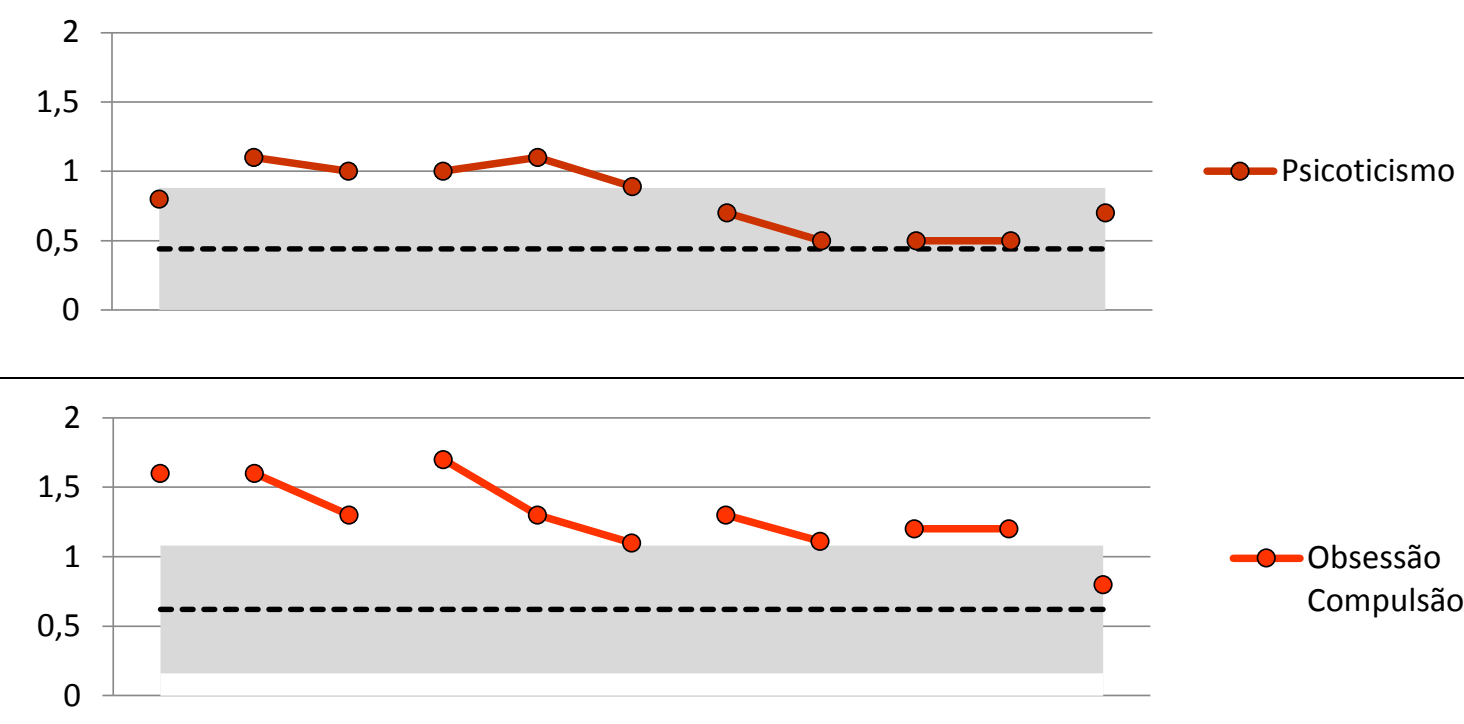

- Obsessão

Compulsão
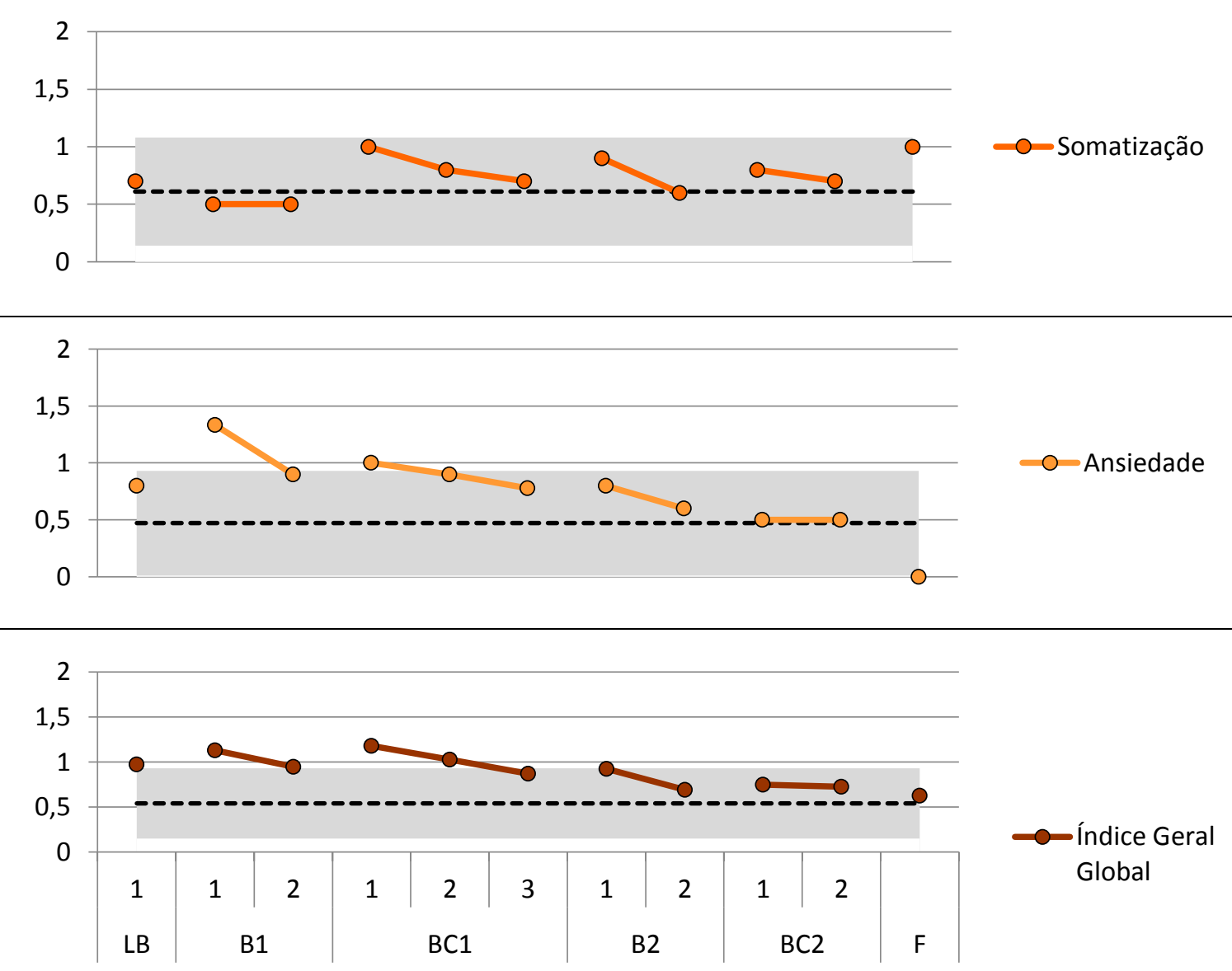

Figura 17: Escores obtidos para cada uma das dimensões medidas pela Escala de Avaliação dos Sintomas (Laloni, 2001) e o índice geral global da cliente Sol. A escala foi aplicada 11 vezes e a figura indica o momento do delineamento experimental no qual a aplicação foi realizada. A linha tracejada em cada quadro indica a média da população para o sexo feminino em cada uma das dimensões e a área em cinza, o intervalo de desvio padrão. F corresponde a follow-up. 


\section{C.2.3) $\mathrm{O} 1$ e $\mathrm{O} 2$}

Assim como feito anteriormente para a cliente Dora, serão analisadas as emissões de Os de Sol ao longo do delineamento experimental. Apesar da cliente apresentar melhoras em seus comportamentos problema e de melhora ao longo do processo terapêutico, evidenciadas pelo registro externo, estas não ocorreram ao longo do delineamento experimental e por isso faz-se interessante investigar as emissões de $\mathrm{O} 1$ e O2 dentro da sessão para aumentar a compreensão do processo da cliente.

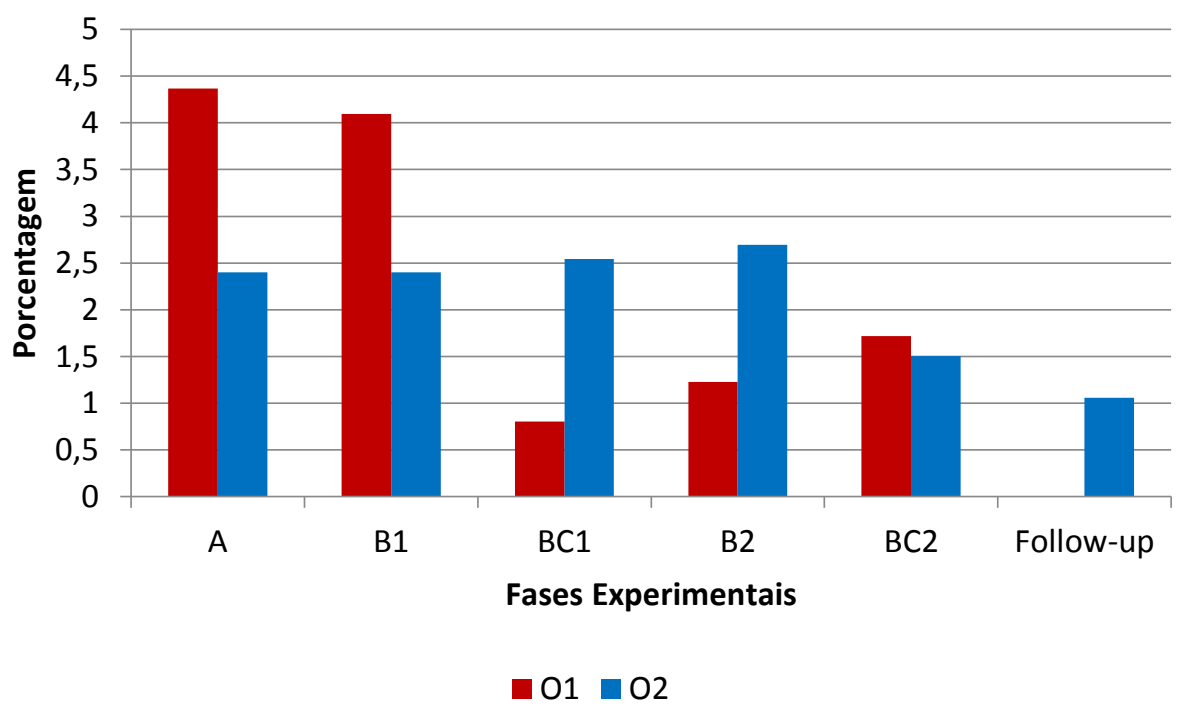

Figura 18: Porcentagem de emissão das categorias referentes a $\mathrm{O} 1$ e $\mathrm{O} 2$, dentre todas as respostas emitidas pela cliente Sol, por fase experimental.

Na Figura 18, observa-se que a porcentagem de emissões de O1 apresentou-se elevada nas duas primeiras fases experimentais do delineamento (Fases A e B1) sofrendo uma grande queda nas fases subsequentes. A Fase BC1 foi a fase na qual tais emissões apresentaram menor porcentagem sofrendo um leve aumento nas fases seguintes, mas permanecendo consideravelmente mais baixa na última fase do delineamento (Fase BC2) do que a apresentada na primeira (Fase A). Por sua vez, a porcentagem de emissões de $\mathrm{O} 2$ permaneceu constante durante a maior parte do delineamento, desde a Fase A até a Fase B2, sofrendo um leve aumento nessa última fase. Já na Fase BC2, a última do delineamento, observa-se uma queda em sua porcentagem de emissão, apresentando-se mais baixa do que a observada logo no início do experimento. Tal dado coincide com aquele observado e comentado anteriormente a respeito da queda na porcentagem de emissão de CCR2 e de análises sobre melhoras nessa mesma fase experimental (em função da mudança de foco da sessão para questões de peso corporal). Ainda, 
na sessão de follow-up, observa-se que ocorreram apenas emissões de $\mathrm{O} 2$, ainda que com uma porcentagem mais baixa do que nas fases experimentais.

\section{C.2.4) Episódios de Os}

Para a cliente Sol também foram analisados os Episódios de $\mathrm{O} 1$ e O2, tal como já descrito para a cliente Dora.

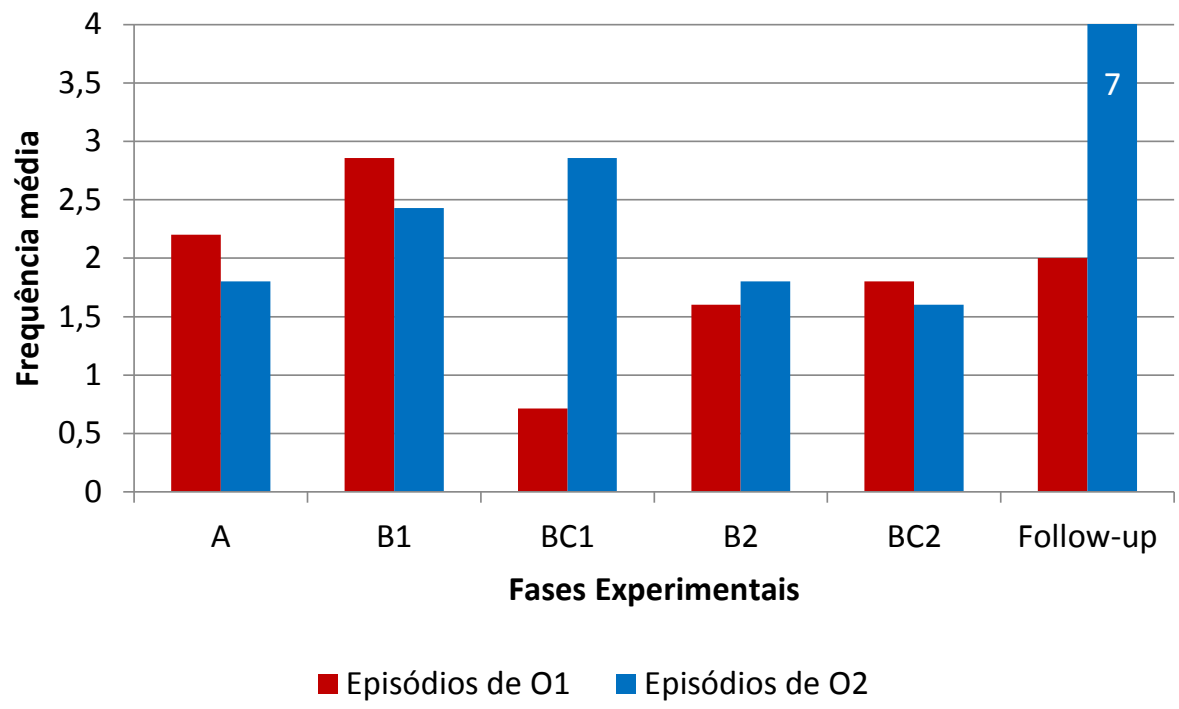

Figura 19: Frequência média de emissão de Episódios de $\mathrm{O} 1$ e Episódios de $\mathrm{O} 2$ detectados nas falas da cliente Sol, por fase experimental. Repetições de uma mesma resposta emitidas durante a semana não foram contabilizadas. Relatos de problema ou melhora emitidos durante a semana, mas que são parte de um CCR também foram contados.

É interessante observar que os Episódios de O1, apresentados na Figura 19, mostram-se bastante semelhantes aos dados de $\mathrm{O} 1$ apresentados na Figura 18. As diferenças ocorrem apenas nas duas últimas fases (Fases B2 e BC2), pelo aumento evidenciado na Figura 19 ser maior do que o evidenciado na anterior. Porém, o mesmo não acontece em relação aos Episódios de $\mathrm{O} 2$. Ao invés de permanecerem constantes ao longo das quatro primeiras fases do experimento, é observado um sucessivo aumento de Episódios de O2s das Fases A até a BC1, voltando a diminuir na Fase B2. Nessa Fase, os Episódios de O2s são apresentados com a mesma frequência média com que tinham sido apresentados na Fase A (1,8 ocorrências) e diminuem ainda mais (para 1,6) na Fase BC2. Tal dado é mais uma evidência de que houve uma piora no comportamento de Sol ao final do experimento. Porém, na sessão de follow-up, a frequência de emissões de Episódios de $\mathrm{O} 2$ aumentou consideravelmente, ultrapassando o dobro do que já havia sido observado até então. 


\section{C.2.5) Relatos e impressões clínicas}

Os dados dos comportamentos de Sol apresentados pelos instrumentos de coleta sobre os comportamentos emitidos fora de sessão parecem contraditórios em alguns aspectos. Enquanto o EAS-40 indica melhora do comportamento de Sol em todas as dimensões analisadas, o registro externo realizado pela cliente não indica alteração durante o delineamento experimental e os dados de Os indicam queda nos $\mathrm{O} 1 \mathrm{~s}$, ainda que com uma piora em relação aos $\mathrm{O} 2 \mathrm{~s}$ na fase final. Além disso, tanto cliente como terapeuta identificam e vinham descrevendo progressos feitos pela cliente fora de sessão, analisando constantemente a superação de muitas dificuldades dela relacionadas aos comportamentos problema descritos. Uma das razões pela qual ambas acreditaram que mudanças importantes já estavam acontecendo durante o delineamento era não apenas pelas respostas do dia a dia relatadas por Sol, mas também pela grande diferença na forma como as pessoas em seu ambiente passaram a lidar com ela, diminuindo drasticamente os abusos e cobranças indevidas que faziam em relação a ela.

Algumas sessões depois de interrompido o delineamento experimental, na sessão correspondente a Sessão 4 Pós-Experimental (ou seja, depois das melhoras pós-experimentais já terem começado a aparecer nos registros) foi discutido com Sol sobre as possíveis razões para o registro não refletir as melhoras terapêuticas identificadas pela terapeuta e cliente. A cliente descreveu que tinha clareza do que estava sendo registrado, o que corrobora a percepção da terapeuta sobre a precisão do registro, mas a cliente relatou acreditar que as principais mudanças que vinha realizando não eram exatamente referentes ao que estava sendo registrado. Ela acreditava que, durante o delineamento, ainda não conseguia negar quando alguém lhe pedia por algo, mesmo quando não queria fazê-lo, mas que tinha conseguido parar de oferecer ajuda aos demais quando na realidade não era essa sua vontade. O comportamento de parar de oferecer ajuda quando não queria fazê-lo, poderia ter sido registrado na categoria de Respostas reforçadas positivamente, produzindo reforçadores mais diretos a ela ${ }^{18}$, pois possivelmente algum comportamento incompatível com o oferecimento de ajuda estaria sendo emitido. Porém, acredita-se que Sol não incluiu alguns comportamentos em seu registro, possivelmente por não estar entendendo a ampla classe funcional que envolvia tais respostas, apesar de ter clareza do registro em relação a algumas topografias.

Assim, ao observar o registro parece que houve uma melhora atrasada em relação ao delineamento, ocorrendo só depois de finalizado o mesmo, mas na verdade possivelmente esse

\footnotetext{
${ }^{18}$ Fez algo por você ao invés de fazer pelo outro.
} 
dado estava relacionado a uma compreensão, por parte de Sol, incompleta sobre seus comportamentos problema e de melhora. Sendo que as respostas com topografias que ela reconhecia ocorreram apenas mais tarde, como se durante a pesquisa estivessem sendo modeladas respostas por aproximações sucessivas, até que passaram a ser emitidas aquelas registradas pela cliente.

Na sessão de follow-up, a cliente descreveu algumas melhoras pelas quais vinha passando que podem exemplificar e corroborar as impressões acima. Na ocasião, estava passando por um período de extremo stress no trabalho, lidando com clientes difíceis, mas tinha começado a trabalhar com vendas (logo depois de encerrado o período experimental), o que para ela era grande avanço, já que tinha que interagir com diversas pessoas que não conhecia ${ }^{19}$ :

C: Porque pra mim tava muito nítido assim... a semana péssima, que eu... que... eu tava passando. Eu tive muitas vezes vontade de largar tudo e sair correndo. E ficava o tempo inteiro pensando "Eu não quero isso, não quero tá aqui, e eu não quero mais fazer isso, e não aguento mais”, coisas que eu sempre tive. Mas eu ficava o tempo inteiro pensando o quão grata eu sou a você de... que eu tenho certeza que o... com certeza eu taria muito pior se não fosse todo esse trabalho que você me ajudou, e que não taria trabalhando com as vendas ao mesmo tempo, não taria aceitando isso da forma como eu to aceitando mesmo com todo esse cansaço.

T: Estaria lutando contra coisas que não te ajudariam.

C: Isso. Tenho muito problema com essa não aceitação das coisas erradas, e eu sei que eu to melhorando, e o fato deu... deu ter hoje esse momento aqui também me deixou muito mais tranquila, então assim, pensei muito no quão grata eu sou. Muito mesmo. (...)

T: Eu vejo toda essa mudança, você sabe, e... e poder fazer parte disso é muito gostoso pra mim, é muito importante, e é muito gostoso ver que você tá dessa forma, você sente que de fato tem ajudado, e... e te ver falando isso, expressando isso, é ainda mais especial, porque eu sei que pra você isso é difícil também. Eu acho que você conseguir falar isso dessa forma tão... tão bonita... Uau! (...)

C: E eu venho assim, pensando assim o quanto pra mim ia fazer muita diferença te falar isso. (...)

T: É muito especial pra mim que você esteja falando. Eu imagino que pra você é importante falar, e pra mim é muito importante ouvir você se expressando desse jeito, ver a nossa relação gostosa desse jeito, e é especial pra mim. Obrigada por me contar que você tem se sentido assim. (...)

\footnotetext{
${ }^{19}$ Foram cortados alguns trechos do relato por ser bastante longo, deixando aqueles que refletem a ideia principal aqui passada.
} 
C: É. Eu acho que se não fosse tudo isso, não sei o que seria de mim. O fato deu nem lembrar mais daquela questão... eu lembrei ontem e nem me incomodou, tá ficando tudo tão no passado coisas ruins, e... coisas que tão me deixando tão estressadas do presente, mas... minha vida tá tão seguindo que... não tem preço isso pra mim. Isso pra mim tem um valor muito grande. (...) Tem algumas coisas que J. (amiga) fala comigo, e... poucas coisas que ela me fala assim eu vejo pontos de mudança, ela nota, assim. Então, eu sei que tudo isso é bem concreto mesmo, e cada dia eu acredito mais.

E a cliente segue reconhecendo as melhoras e fazendo planos futuros (que são considerados como CCR2 para ela).

\section{C.3) Resultados externos e decorrentes reflexões}

Diante dos dados apresentados sobre o comportamento externo das clientes, é possível encontrar certas similaridades entre as duas.

Em ambos os casos, tem-se que a análise isolada dos registros externos realizados pelas clientes indica uma melhora terapêutica, mas é insuficiente para afirmar que houve tal melhora. No caso de Dora, a diminuição das agressões é bastante sutil, enquanto Pensamento ruminativo aumentou em frequência ao longo do experimento, sendo que esse é um problema de magnitude e consequências menores do que as agressões. No caso de Sol, o registro indica melhora nos comportamentos da cliente apenas depois de encerrado o experimento, sugerindo uma melhora atrasada ou mais provavelmente uma compreensão incompleta sobre a classe de respostas registradas por parte de Sol. Porém, em ambos os casos, supõe-se que houve melhora terapêutica pela soma dos dados apresentados: outras formas de registro (por escala e pelo observador externo) e os dados referentes aos Os e Episódios de Os, no caso de Dora; e pelo EAS-40, e dados referentes aos Os e Episódios de Os, no caso de Sol. Além disso, em ambos os casos, tanto clientes como terapeutas descrevem a ocorrência de melhoras em momentos diversos da terapia, tanto durante como depois de ter sido encerrado o experimento. Os dados de follow-up coletados, tanto pela análise de uma sessão, como pela aplicação do instrumento EAS-40, indicam ainda a manutenção dessas melhoras. Desse modo, a soma de indícios, provindo de variadas fontes, sugerem que aconteceu tal melhora terapêutica.

Kazdin (2009) chega a apontar que nenhuma forma de medida sistemática substitui o julgamento clínico, que é capaz de capturar informações que instrumentos nem sempre captam. O autor aponta que isso não reduz a importância de se ter medidas sistemáticas sobre os progressos da terapia, já que ocorrem limitações por julgamentos, percepções e memórias, de modo que as medidas sistemáticas provêm uma complementação necessária às impressões clínicas. Sendo assim, julga-se na presente pesquisa que a soma dos dados obtidos pelas 
diferentes formas de coleta, mesmo não sendo robustos individualmente, complementam satisfatoriamente as percepções clínicas mencionadas de terapeuta e clientes.

Vale ressaltar que no caso de ambas as clientes a maior mudança parece ocorrer nas emissões de $\mathrm{O} 1$ e não nas emissões de $\mathrm{O} 2$. Ou seja, parece ter havido maior diminuição nos comportamentos problema do que aumento nos comportamentos de melhora. Isso é evidenciado tanto nas análises sobre Os como sobre Episódios de Os. É curioso observar que algumas das formas de registro de ambas as clientes parecem indicar um aumento nos comportamentos problema na Fase B1 em comparação ao que vinha sendo emitido até então, ou seja, quando foi introduzida a FAP. Esse dado é indicado pelo registro externo por frequência de Dora e pela porcentagem de emissão de seus O1s; bem como pelo registro externo de Sol, seus dados do EAS-40 e pelos dados dos Episódios de O1.

Como pode ser observado, boa parte das conclusões sobre as melhoras das clientes está sendo pautada na análise de Os e de Episódios de Os. Sabe-se que muito pode ser questionado a respeito dessas formas de análises. Tais questionamentos podem surgir em relação a 1) a baixa quantidade de emissões de Os observada em sessão (Busch et al., 2010); 2) a hierarquia do sistema FAPRS encobrindo relatos de Os, conforme mencionado por Busch et al. (2010); 3) a influências do terapeuta sobre a quantidade de relatos de melhora ou piora que pode ocorrer na sessão; 4) a precisão do relato realizado pelo cliente correspondendo ou não ao que de fato aconteceu externamente à terapia. Abaixo serão comentados cada um desses questionamentos em relação a presente pesquisa.

Como já observado por Callaghan et al., (2003) e Busch et al. (2010), poucas emissões de Os têm sido observadas nas sessões de pesquisas em FAP, não sendo possível obter a partir daí indicativos robustos de melhora ou piora. De fato, na presente pesquisa são observadas poucas ocorrências de relatos em sessão, mas, da mesma forma, foram observados poucas ocorrências de comportamento externo da cliente Dora, assim como na maioria das pesquisas produzidas na área (Kanter et al., 2006 e Landes et al. 2013). No caso de Sol, a quantidade de registro externo coletada é maior do que a quantidade de emissões de Os em sessão, aproximando-se mais do dado observado por Lizarazo et al. (no prelo). Assim, há controvérsias a respeito da superioridade do registro externo em relação às análises de Os em função da quantidade de respostas observadas, pois aparentemente nem sempre é possível coletar mais dados através do registro do que através da análise realizada sobre o relato do cliente.

Ainda, como apontado por Busch et al. (2010), a forma como o sistema FAPRS prevê a hierarquia das categorizações, pode prejudicar sua contagem, "encobrindo" o dado sobre comportamentos extra sessão, sendo um dos responsáveis pelas poucas emissões de relatos. 
Nesse sentido, acredita-se que a contagem de Episódios de Os realizada na presente pesquisa é um avanço em relação a simples porcentagem de Os utilizadas em pesquisas anteriores. Isso porque por ser uma contagem independente das demais categorias do sistema FAPRS, não se corre o risco de problemas frente à hierarquia do sistema influenciar em sua frequência, deixando portanto o dado mais fiel ao que de fato ocorreu fora da sessão do que a análise da porcentagem de Os. Porém, ao menos na presente pesquisa, tal contagem independente do sistema FAPRS não gerou um aumento na quantidade de dados obtido, apesar de apresentar um dado mais fiel ao que ocorreu externamente à sessão do que a análise da porcentagem de Os.

Ainda, pode ser questionado o fato da quantidade de emissões de Os (bem como dos Episódios de Os) poder variar não apenas de acordo com problemas e melhoras ocorridos no dia a dia das clientes, como também de acordo com evocações e consequenciações dados pela terapeuta a essas falas. Desse modo, pode-se obter diferentes quantidades de relatos de problemas ou de melhoras a depender da condução da terapia, não sendo fiel ao que de fato ocorreu fora de sessão. Porém, tal possibilidade é reduzida na medida em que as análises sobre Os foram realizadas posteriormente ao final da condução do delineamento experimental, o que reduz ao menos em parte a chance de grandes influências sobre o relato de problemas ou melhoras. Além disso, mais uma vez a análise de Episódios de Os mostra-se mais adequada do que a porcentagem de Os, já que acredita-se que tal análise reduz a influência do terapeuta sobre o dado. Isso porque mesmo que a terapeuta evocasse a descrição desses episódios, simples repetições dos mesmos episódios não foram contabilizadas, sendo necessário, portanto reais ocorrências desses episódios para sua contagem. A única chance do comportamento da terapeuta influenciar esses relatos é ao impedir ou ao encorajar o relato ou menção deles em sessão, não sendo possível influenciá-lo ao simplesmente prolongar a quantidade de falas a respeito de um episódio ocorrido.

Por fim, pode-se questionar se o relato que o cliente faz é realmente confiável, mesmo que sua imprecisão não ocorra por influência do terapeuta. Acredita-se aqui que nenhuma das duas clientes esteve distorcendo os relatos de modo a dar impressões equivocadas à terapeuta. É bem verdade que ambas apresentaram dificuldades em reconhecer melhoras em alguns momentos da terapia, mas com a intervenção da terapeuta, apontando o ocorrido, ambas reconheciam o que estava sendo dito. Assim, considera-se que nos momentos em que o relato das clientes não era fiel ao que havia ocorrido, indícios apareceram, sendo esse um problema mais de reconhecimento das situações do que de distorções em si, não havendo maiores 
motivos para se acreditar que as clientes estiveram de alguma forma manipulando o que era dito à terapeuta sobre fatos de seu dia a dia.

Ainda, em relação à presente pesquisa, vários problemas podem ser levantados ao registro externo realizado pelas clientes, que não demonstraram grandes melhoras, contrariamente às impressões clínicas já mencionadas.

Diferentemente de pesquisas anteriores, no presente trabalho optou-se por não entrar em contato com o registro externo das clientes durante o período de coleta de dados. Como já mencionado anteriormente, nas pesquisas de Kanter et al. (2006), Landes et al. (2013) e Lizarazo et al. (no prelo) os terapeutas acompanhavam o registro e discutiam com o cliente em sessão como tal registro estava sendo feito durante todo o delineamento, corrigindo possíveis enganos nos registros durante a coleta de dados. Ou seja, optou-se por modelar o repertório de registro dos comportamentos externos mesmo durante o delineamento experimental. De fato, a bibliografia sobre auto registro para acompanhamento das melhoras dos clientes recomenda que isso seja feito para aumentar a probabilidade de se ter registros precisos (Bohm \& Gimenes, 2008, Korotitsch \& Nelson-Gray, 1999). Tal procedimento, apesar de ter gerado dados muito mais claros nas demais pesquisas do que nessa a respeito da melhora extras sessão, gerou também dados fortemente influenciados pelo terapeuta.

O fato é que, na presente pesquisa, pelo relato pós-experimental das clientes sobre seus comportamentos de registrar, ambas teriam se beneficiado muito de auxílios com o registro ao longo da coleta de dados, clarificando o que deveria estar sendo registrado, tornando-o muito mais preciso. Mas o mesmo tipo de questionamento poderia ser feito sobre as influências que a terapeuta poderia estar exercendo sobre tais registros. Optou-se no início da coleta por uma maior neutralidade que acabou por gerar uma grande imprecisão no dado. Na presente pesquisa ainda, não era recomendado dispender um grande período de treinamento na especificação dos comportamentos registrados antes de iniciada a coleta de dados, pois isso possivelmente implicaria em uma profunda e ampla análise do comportamento das clientes com as mesmas, que era, na realidade a variável dependente a ser controlada na presente pesquisa.

Além disso, outro fator que pode ter contribuído para os problemas encontrados aqui com o registro das clientes é o tempo de duração da coleta de dados. Bohm e Gimenes (2008) recomendam que pausas sejam feitas nos registros durante algumas semanas para aumentar o engajamento do cliente na atividade, o que não foi feito aqui. Korotitsch e Nelson-Gray (1999), chamam ainda a atenção para o caráter aversivo que o registro pode apresentar para algumas pessoas e o prejuízo que isso pode trazer para o registro, tal como foi observado no caso de Dora. 
Diante das dificuldades encontradas, pode-se colocar em dúvida se seria preferível dados coletados pelos clientes externamente à terapia em relação às falas ocorridas em sessão. Afinal, as dificuldades e falta de uniformidade na forma desse tipo de coleta, mencionadas por Bohm e Gimenes (2008), Hatfield e Ogles (2004), Korotitsch e Nelson-Gray (1999), Lambert e Hawkins (2004) e Starling (2010), falam a favor desses questionamentos.

Outros vieses também podem ocorrer como omissões dos clientes sobre determinado assunto ou acontecimento ou mesmo ênfase em outros, que podem alterar tanto o relato em sessão como o registro externo. Desse modo, parece certo pensar que nenhuma dessas possibilidades é uma forma realmente imparcial e ao mesmo tempo precisa de registro. Frente a essas questões, faz-se importante explicitar que não defende-se aqui a superioridade do dado de pesquisa proveniente dos relatos em sessão sobre os registrados fora de sessão, mas defendese que estes pareceram mais fieis na presente pesquisa do que aqueles coletados pelas próprias clientes. Ao menos em nosso país, é pouco frequente a solicitação de registros sobre o próprio comportamento para ser utilizado como dado em terapia, seja em pesquisa ou não, de modo que os relatos dos clientes em sessão são geralmente os principais dados em terapia.

Parece importante que pesquisas continuem investigando melhores formas de coleta de informações extra sessão, em especial quando se trata de comportamentos específicos de cada cliente e não outros mais gerais, não idiossincráticos, que são obtidos através de instrumentos padronizados, como no caso do EAS-40.

O Goal Attainment Scale (GAS, Schlosser, 2004 - instrumento traduzido para o português por Starling, 2010), por exemplo, é um instrumento criado para registro de comportamento externo, definido idiossincraticamente, no qual é iniciado com uma forma padronizada de se definir os comportamentos a serem registrados. Através de dez passos, tal instrumento deveria preparar o cliente para a compreensão e precisão do registro. Tal instrumento poderia ser uma boa opção para uso em pesquisas que visam a coleta de informações externas, não fosse uma característica que torna-se um grande viés. A GAS, por gerar a produção tão detalhada na definição dos comportamentos a serem registrados é considerado como uma forma de intervenção por si só e não apenas uma forma de registro, o que pode acabar por ser uma variável a mais em qualquer pesquisa que venha a utilizá-lo.

De qualquer modo é preciso que ainda se invista na melhora da coleta do dado externo, seja em 1) formas de preparar o cliente para registrar seu próprio comportamento de forma precisa e imparcial (até onde é possível) ou seja em 2) formas de identificar claramente e de forma também imparcial informações sobre melhoras e pioras provindas do relato dos próprios clientes em sessão. 
No caso da primeira, quanto a melhorar o preparo do cliente para o registro de seu próprio comportamento, autores que investigaram registros realizados por clientes, definiram já algumas características importantes do auto registro que devem ser seguidas, tais como ter respostas bem definidas, treino de como registrar, checagens esporádicas sobre o que está sendo registrado, entre outros (Bohm \& Gimenes, 2008; Korotitsch \& Nelson-Gray, 1999). Além disso, levanta-se aqui a possibilidade de se ter uma terceira pessoa participando da pesquisa, que seja cego para a hipótese da pesquisa conduzida, mas a par da conceituação do caso, que auxilie o cliente no registro preciso de seu comportamento, reduzindo a influência da hipótese da pesquisa sobre o registro. Nesse caso, é preciso ser cuidadoso para que tal pessoa que deveria apenas auxiliar no registro não se torne, ainda que parcialmente um segundo terapeuta do cliente. Sugere-se, então, que alguns limites sejam estabelecidos como um limite de tempo curto de contato entre cliente e essa pessoa, no qual não é possível haver grandes aprofundamentos dos temas, ou limites sobre assuntos que devem ser discutidos, a fim de se reduzir interferências de uma variável a mais na pesquisa. Outra possibilidade é desenvolver novas formas de registros, através da tecnologia existente atualmente, como o desenvolvimento de aplicativos de celular, por exemplo, que possibilitem um registro menos custoso e em alguns casos, até mais reforçador (para um exemplo desse uso de tecnologia ver Bedesem \& Dieker, $2014)^{20}$.

Sobre como melhorar a identificação de melhoras ou pioras a partir do relato do cliente, sugere-se aqui a análise posterior de Episódios de Os, realizada na presente pesquisa e relatada acima. Acredita-se que essa forma de análise traz mais vantagens do que a de análise por porcentagem de emissão de Os, por sofrer menos influências da hierarquia do sistema FAPRS e mesmo de incentivos ou não do terapeuta para falar de determinado assunto.

Serão analisadas a seguir as possíveis influências que a condução das sessões em suas diferentes fases experimentais pode ter tido sobre as mudanças nos comportamentos extra sessão indicadas a partir da contagem de Episódios de Os.

\footnotetext{
${ }^{20}$ Atualmente esforços vem sendo feitos para se desenvolver aplicativos a serem utilizados por clientes para registro do comportamento externo nas pesquisas em FAP em dois importantes centros de pesquisa da área: no Center for the Science of Social Connection, na Universidade de Washington, em Seattle, sob a direção do Prof. Dr. Jonathan Kanter; e na Universidade Federal do Paraná, sob a coordenação da Profa. Dra. Jocelaine Martins da Silveira.
} 


\section{D) Relações entre respostas intra e extra sessão}

Pode se questionar ainda acerca das possíveis relações entre respostas emitidas dentro de sessão e os progressos ocorridos no dia a dia das clientes. Ou seja, serão rapidamente investigadas possíveis evidências de que os progressos no dia a dia estiveram relacionados com o processo terapêutico.

Como apresentado acima, a única forma de coleta de dados externos que apresentou alguma relação de progressos de Dora com a mudança entre fases experimentais foram aqueles levantados pelo instrumento EAS-40. Todos os demais dados externos indicam melhoras (algumas bastantes modestas, como discutido) que ocorreram ao longo do delineamento experimental sem as esperadas variações de acordo com a inserção da VD que deveriam ocorrer no caso das melhoras acontecerem em função dessa. É interessante questionar a razão do EAS40 da cliente Dora ser o único instrumento a demonstrar um indicativo nesse sentido, mas na realidade esse dado isolado não é forte o bastante para sustentar argumentos em favor da influência da Regra 5 sobre melhoras externas da cliente. No caso da cliente Sol, nenhum dos dados externos apresentados indicaram influência da Regra 5 sobre os comportamentos externos. O fato de não ocorrer esse tipo de sistematicidade de mais melhoras ocorrendo nas fases com ou sem a VD indica que a emissão de Regra 5 não pareceu interferir diretamente nas melhoras que ocorrem extra sessão, ao menos não de forma expressiva ou que se refletisse nos dados coletados.

Porém, considerou-se importante ainda especular o que levou a tais melhoras extra sessão. Para isso, pode-se fazer uma análise adicional, unindo-se dados já apresentados anteriormente. Assim, na Figura 20, estão sendo apresentados os dados referentes a porcentagem de emissão de CCR1 e CCR2 em cada fase experimental, em conjunto com a frequência média de emissão de Episódios de O1s e O2s. Assim é possível comparar se aumentos e diminuições de melhoras ou problemas dentro de sessão corresponderam ou não a aumentos e diminuições de melhoras ou problemas fora de sessão.

Na Figura 20 observa-se que, para a cliente Dora, aumentos e diminuições de CCR1s nem sempre foram acompanhados de aumentos e diminuições de Episódios de $\mathrm{O} 1$ e o mesmo ocorreu na comparação entre CCR2s e Episódios de O2. Porém CCR1 e Episódios de O1 são predominantes em relação a CCR2 e Episódios de O2, respectivamente, apenas na Fase A, sendo que já na Fase B1, antes da introdução da Regra 5, há uma inversão nessa predominância, que permanece até o final do experimento. Esse dado indica que as melhoras intra e extra sessão ocorreram em momentos semelhantes para essa cliente, quando foi introduzida a FAP, mesmo 
sem a introdução ainda da Regra 5 (Fase BC1). A partir dos dados observados, pode-se concluir que a Regra 5 influenciou apenas o comportamento de analisar de Dora e não influenciou seus comportamentos problema e de melhora nem dentro nem fora de sessão. De fato, essa cliente chegou a descrever em certos momentos o quanto não estava disponível, em especial nos primeiros meses de terapia para ouvir a terapeuta em suas análises ou falas mais longas e o quanto que ouvi-la falar não era seu foco. Assim, possivelmente mesmo quando algumas análises eram feitas, Dora não entrava em contato com as mesmas. Por isso parece que Dora se beneficiou mais do lado experiencial do que do analítico da FAP, sendo provavelmente o primeiro suficiente para que seus comportamentos fossem modificados no dia a dia.

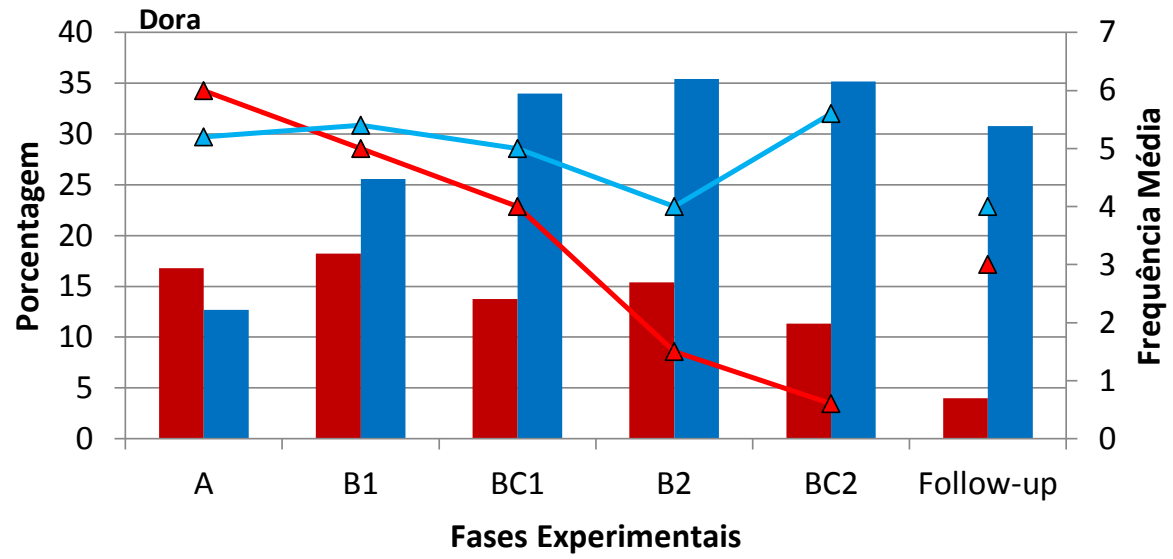

CCR1 CCR2 $\triangle$ CEpisódios de $01 \triangle$ Episódios de $\mathrm{O} 2$

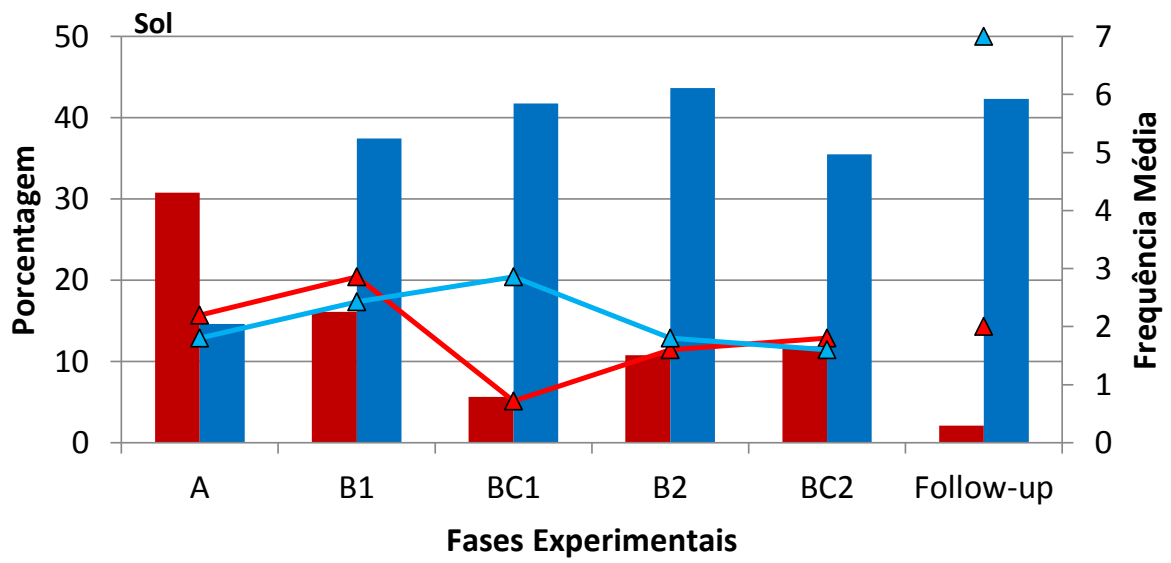

$\square$ CCR1 CCR2 $\triangle$ Episódios de $01 \triangle \triangle$ Episódios de O2

Figura 20: Porcentagem de emissão de CCR1 e CCR2 (barras - escala à esquerda), dentre todas as respostas emitidas pelas clientes e frequência média de emissão de Episódios de $\mathrm{O} 1$ e Episódios de $\mathrm{O} 2$ (linhas - escala à direita) detectados nas falas das clientes, por fase experimental e sessão de follow-up. As escalas dos gráficos são individualizadas para cada participante. 
No caso de Sol, há mais correspondência entre aumentos e diminuições de CCR1 com Episódios de O1 e CCR2 com Episódios de O2 do que com a cliente Dora. Há apenas duas exceções: quando CCR1 diminuiu em porcentagem de emissão da Fase A para a Fase B1, ao mesmo tempo em que Episódios de O1 aumentaram em frequência; e quando CCR2 aumentou levemente da Fase BC1 para a Fase B2, enquanto a frequência de emissão de Episódios de O2 diminuiu. Vale destacar que a diminuição de Episódios de $\mathrm{O} 2$ na última fase do experimento corresponde a diminuição de CCR2 nessa mesma fase, e ao aumento de análises emitidas pela cliente sobre seus comportamentos problema, o que pode indicar que houve uma piora nas respostas dessa cliente tanto dentro como fora de sessão ao final do delineamento experimental, conforme apresentado anteriormente. Lembra-se aqui que tal piora dentro de sessão está sendo atribuída à mudança de foco da terapia ocorrida, quando a cliente passou a falar mais sobre um tema bastante difícil relacionado a seu peso corporal. Entretanto se faz curioso observar que a piora nos Episódios de $\mathrm{O} 2$ mostrou-se mais evidente do que nos CCRs já na Fase B2, dando a impressão de que tal piora começou fora de sessão para depois afetar seus comportamentos em sessão, ao mudar o foco da terapia de Sol.

Além disso, para a cliente Sol, a emissão de melhoras fora de sessão, tal como apresentada pelo presente dado, apenas superou as emissões de problemas dentro de sessão na Fase BC1, sendo que dentro de sessão isso já havia ocorrido na Fase B1. Pode-se questionar se a melhora fora de sessão ocorreu em função das emissões de análises que começaram a ocorrer nessa Fase BC1. Porém, devido aos outros dados apresentados anteriormente contra essa hipótese e devido ao fato da cliente ter apresentado piora na Fase BC2, na qual também ocorreram mais análises, pode-se também questionar se as melhoras ocorridas fora de sessão foram, na realidade, um efeito atrasado das melhoras ocorridas dentro de sessão já na fase anterior.

Desse modo, observa-se que a Regra 5 gerou um impacto no comportamento de Sol analisar, principalmente quanto ao ocorrido dentro de sessão; além disso a correlação apresentada para Sol entre Regra 5 e análises emitidas por ela foi maior do que para a cliente Dora, como apresentado anteriormente na Tabela 9. Análises para ela já eram mais frequentes do que para Dora, mesmo nas Fases A e Bs, mas as análises da terapeuta influenciaram principalmente no conteúdo das análises da cliente, focando-as dentro da sessão.

Na realidade observa-se que grandes mudanças na quantidade de Os e Episódios de Os foram observadas com a cliente Sol, quando foi introduzida a Fase BC1, enquanto que com a cliente Dora, estas vieram ocorrendo gradualmente de fase a fase. Porém, faz-se difícil confirmar uma influência das análises sobre as melhoras externas devido ao fato do mesmo 
efeito não ser observado nas fases posteriores do delineamento. Assim, não há indícios suficientes de que tal comportamento de análise tenha afetado melhoras ou pioras emitidas por Sol dentro ou fora da sessão. Ao contrário, parece que as melhoras atingidas em sessão podem ter tido um maior impacto nas melhoras extra sessão do que as análises.

\section{E) Reflexões adicionais sobre o papel das análises na FAP}

Considera-se que há dois processos distintos na FAP quando aplicada de forma completa: um de reconhecimento, evocação e modelagem de CCRs (experiencial - Regras 1 a 4) e outro de análise das contingências que envolvem essa modelagem (analítico - Regra 5), sendo que o primeiro deles é considerado o principal, que caracteriza a FAP, sendo o segundo um complemento.

Como já foi discutido na literatura, acredita-se que análises de contingência realizadas em sessão possam ser mais ou menos importantes, a depender do cliente, da queixa, do momento do processo, da história da pessoa com seguimento de regras entre outros, como sugerido por Meyer, 2004. Vale salientar que os efeitos das análises investigadas no presente trabalho, tanto as feitas por terapeuta como as feitas por cliente, não dizem respeito ao mesmo efeito que análises possivelmente apresentam em terapias nas quais o foco de trabalho encontra-se no desenvolvimento de análises, como realizada por tanto tempo em terapias comportamentais. Skinner (1989) e Guilhardi (1997), por exemplo, descrevem o processo da terapia como tendo o objetivo de aumentar a capacidade do cliente de descrever as contingências que controlam seu comportamento e influenciá-las. Zamignani (2007), por sua vez, chegou a afirmar que interpretações, solicitação de reflexão, recomendação e aprovação são comportamentos do terapeuta que produzem, mudanças no processo terapêutico.

Garfield (1995), por sua vez, questiona o fato de que diferentes abordagens de terapia, fazem diferentes interpretações sobre o comportamento do cliente mas são similarmente efetivas. $\mathrm{O}$ autor discute que, possivelmente o conteúdo das análises não é o mais relevante, mas que o cliente acreditar que o terapeuta é capaz de entender seu comportamento tem um impacto terapêutico importante. Tal discussão leva a reflexões de que talvez, de fato, as análises não sejam o aspecto mais importante de uma terapia que não as tem como foco, mas que estas podem servir para suplementar algum aspecto.

O foco de uma terapia FAP, por outro lado, é diferente desse descrito pelos autores acima. Sua preocupação está em modelar repertório de melhora em sessão a fim de generalizá-lo para fora de sessão. Para isso análises funcionais são utilizadas 1) o tempo todo como embasamento 
para a modelagem que está sendo feita e, 2) segundo a literatura (Kohlenberg \& Tsai, 1991; Tsai et al., 2009), como forma de clarear para os clientes as contingências que controlam seu comportamento problema e especialmente de melhora, a fim de aumentar a probabilidade de generalização dessas melhoras de dentro para fora da terapia. O objetivo do presente estudo foi, portanto, investigar essa segunda função que as análises teriam em uma terapia FAP, investigando se esse complemento é necessário para que melhoras ocorram dentro e fora de sessão. Afinal, uma terapia com foco em análises deve ser diferente de uma terapia com foco em modelagem de repertório e complementada com análises.

Nos dois casos conduzidos na presente pesquisa, verificou-se que dar exemplos de análises, solicitar ou reforçar análises produziu mais análises no repertório das clientes. Além disso, a análise realizada por Mendes (2014) sobre parte dos dados de Sol indicou que com essa cliente evocar diretamente reflexões da cliente foi a forma mais efetiva de produzir análises. Porém, essas análises não se relacionaram de forma forte e clara com os demais comportamentos (de melhora e piora) de nenhuma das clientes. Os dados aqui apresentados dão fortes indícios de que Regra 5, CCR3 e O3 não foram necessários para que ocorresse uma melhora terapêutica durante as sessões FAP.

Desse modo, está indefinido ainda qual o papel da Regra 5 e das análises dos clientes na melhora dos mesmos, ao menos na forma como foram feitas aqui. Talvez possa-se supor que as análises tenham uma importância variável também como complemento da parte experiencial da FAP.

Apesar das diferenças mencionadas entre as duas clientes, em nenhum dos casos, a Regra 5, o CCR3 ou o O3 mostrou grande diferença nos resultados alcançados. Isso pode significar que, quando uma terapia FAP está sendo conduzida de maneira persistente, análises sobre esse processo tornam-se irrelevantes ou com baixo impacto, não acrescentando muito aos ganhos alcançados pela parte experiencial do processo.

Como apresentado anteriormente, o aprendizado por regras pode gerar certa insensibilidade às contingências e uma das formas de diminuir essa insensibilidade às contingências criadas pelas regras é sendo capaz de discriminar as contingências presentes. Por outro lado, conforme apontado por Skinner (1969), as regras são especialmente úteis e até mais completas em circunstâncias nas quais as contingências em vigor são fracas. Há dados da pesquisa básica mostrando que regras auxiliam o aprendizado de novas respostas em contextos nos quais as contingências em vigor não são claras; e por outro lado, quando há contingências claras em vigor, a suplementação por regras não gera grande impacto sobre o comportamento 
(Abreu-Rodrigues \& Sanabio, 2004; Matos, 2001; Meyer, 2005). Nas palavras de Matos (2001):

quando as regras são ambíguas mas as contingências são simples, isto é, fáceis de serem discriminadas, os sujeitos as discriminam e passam a agir de acordo com elas; mas se as contingências são complexas e o desempenho exigido é elaborado, os sujeitos podem apresentar um desempenho errático e variável, até, eventualmente ficarem sob controle das contingências em vigor; ou podem formular auto regras a partir de suas experiências passadas com situações semelhantes. Eventualmente, mesmo aqueles que respondem de acordo com as contingências podem vir a formular uma regra sobre seu desempenho (...) e ficar sob controle dessa regra daí por diante. (p. 56)

O fato é que a análise de contingências (regras) pode suplementar o controle pelas contingências em si quando estas não estão claras, tornando-as mais fortes. Assim, como mencionou Skinner (1974) nos versos apresentados na introdução desse trabalho, o ferreiro medieval pode ter tido a descrição de seu comportamento como uma vantagem na medida em que o ajudou a manipular corretamente o fole ou para ajudá-lo a repetir essa forma de responder tempos depois.

No caso da FAP usada de forma sistemática, é possível dizer que as contingências estão claras. Ao se retomar os dados apresentados na Figura 5, constata-se a grande probabilidade de que os CCR2s das clientes tenham sido reforçados com taxas altas de reforçamento, enquanto os CCR1s foram punidos ou colocados em extinção sistematicamente. Os dados de alta correlação positiva entre os CCRs e as respostas dadas a eles (TRB1 e TRB2) também indicam isso. Desse modo, as clientes foram possivelmente submetidas a contingências claras e consistentes, fáceis de serem discriminadas, mesmo que não o fossem descritas. Pelo processo de modelagem ter sido feito de forma constante talvez a necessidade das regras tenha diminuído por elas estarem respondendo às claras contingências do ambiente.

Conforme apontado por Cerutti (1991), Abreu-Rodrigues e Sanabio-Heck (2004) e Meyer (2005), esquemas de reforçamento que tenham regularidade talvez sejam mais facilmente discrimináveis do que os de reforçamento intermitente, indicando que há propriedades discriminativas nas contingências que as facilitam ou as dificultam. Em outras palavras, os diferentes contextos possivelmente tornaram-se óbvios para as clientes da presente pesquisa, levando a diferenciações no responder em sessão. Devido às semelhanças entre os contextos externo e interno à terapia, tais respostas passaram a ser emitidas nesse contexto, mesmo que sem a consequenciação imediata do reforço no ambiente externo. 
Follette et al. (1996) já haviam apresentado essa ideia ao dizerem que

Quando terapeutas respondem contingentemente ao cliente em uma frequência bastante alta, há uma chance muito maior dos clientes aprenderem respostas mais efetivas do que quando esse feedback acontece em intervalos randômicos. Não estamos sugerindo que reforçadores contingentes, naturais ocorrem em sessões de terapia em um intervalo fixo ou de razão fixa. Terapeutas reforçam comportamentos em sessão em um esquema de razão variável. A capacidade desse esquema de manter o responder pode explicar porque as respostas do cliente continuam durante períodos de baixo reforçamento.

Esse é o mesmo esquema com o qual comportamentos são reforçados fora de terapia. Reforçadores naturais em sessão, no entanto, provavelmente ocorrem em esquema de razão variável mais rico do que aquele que ocorre fora de terapia. No nosso modelo da FAP, o terapeuta provê reforçamento em um esquema de intervalo fixo ou de razão fixa no início da terapia para estabelecer novas respostas a uma taxa mais rápida. Com o progresso da terapia, esses reforçadores passam a ser liberados em um esquema mais variável, mantendo o responder do cliente fora de sessão quando outros acabam por falhar quanto ao reforço do novo comportamento do cliente. (p. 635-636) ${ }^{21}$

Desse mesmo modo, as análises elaboradas com clientes em sessão podem vir a servir como um complemento, mas não sendo essenciais (como não o foi no caso de Dora e Sol) para controlar seu comportamento, pelo ambiente terapêutico estar provido de contingências claras e de fácil discriminação, como sugerido por Follette et al. (1996). Vale lembrar que, nas demais pesquisas da área, a Regra 5 também não se mostrou necessária, mas nenhuma delas havia controlado sua apresentação a fim de confirmar seu efeito. Apenas no caso de Geremias (2014) e Lizarazo et al. (no prelo), foi possível observar que estas foram emitidas em baixa frequência.

Tal como sugerido pela proposta da FAP a similaridade funcional existente entre os contextos intra e extra sessão deveria produzir novas respostas, aprendidas em sessão nos contextos de vida diária dos clientes, tendo as regras formuladas como forma de auxílio nessa mudança. Porém, Villas-Bôas et al. (2015) discutiu anteriormente, o quanto tal processo não

\footnotetext{
${ }^{21}$ No original em inglês: "When therapists respond contingently to client behavior at a very high frequency, there is a much greater chance of clients learning more effective responses than when this feedback comes at random intervals. We are not suggesting that contingent, natural reinforcers occur in therapy sessions on a fixed interval or fixed ratio basis. Therapists reinforce in-session behaviors on a variable ratio schedule. The ability of this schedule to sustain responding may explain why client responses continue during periods of lean reinforcement.

This is the same schedule with which behaviors are reinforced outside of therapy. In-session natural reinforcers, however, probably occur on a richer variable ratio schedule than those that occur outside of therapy. In our model of FAP, the therapist provides reinforcers on a fixed interval or fixed ratio schedule early in therapy to establish new responses at a more rapid rate. As therapy progresses, these reinforcers are delivered on a more variable schedule, maintaining client responding out of session when others might fail to reinforce new client behavior."
} 
parece ser simples, já que as contingências do ambiente externo que evocam e reforçam comportamentos problema estão em vigor geralmente por muito tempo e com força suficiente para manter os comportamentos problema, dificultando que as melhoras passem a acontecer fora de sessão pela simples similaridade funcional. Em função dessa dificuldade, levantou-se previamente a hipótese de que as análises poderiam auxiliar alterando a função dos estímulos externos à terapia e aumentando a probabilidade de mudanças (Kohlenberg, Tsai, \& Dougher, 1993; Meyer, 2000, 2004; Villas-Bôas et al., 2015).

Previamente foi discutido o quanto regras podem apresentar a função de alteração de estímulos, além de descrever contingências (Schlinger \& Blakely, 1987). Assim, Villas-Bôas et al. (2015) levantaram a hipótese de que as regras descritas sobre a relação terapêutica poderiam alterar a função de estímulos externos à terapia, auxiliando na mudança de comportamento em outros contextos que não a própria terapia. Os dados aqui apresentados nem confirmam nem tampouco contestam tal hipótese. O que se observou é que possivelmente as regras não foram necessárias para tal alteração de comportamento fora de sessão. No momento então, permanece a pergunta de quais os processos envolvidos nessa alteração de comportamento no dia a dia das clientes.

Ora, se as mudanças comportamentais das clientes não ocorreram através desse processo e nem tampouco pareça razoável se dizer que houve mudanças externas repentinas ao acaso que levaram a essas mudanças, evocando e reforçando novas formas de responder, o que teria então levado às mudanças? O que talvez tenha acontecido é que o fato das contingências em sessão serem claras, pode ter levado a um melhor processo de discriminação no repertório das clientes, facilitando o reconhecimento de determinados contextos fora de sessão, o que levaria a diferenças no responder diário, levando a modificações no ambiente, que levariam a mais mudanças no responder das clientes. Aparentemente, a mudança de comportamento em sessão, realizada através de uma organização consistente de contingências, foi suficiente para levar a alterações fora de sessão. Diante disso, será possível dizer que a "simples" sistematicidade das contingências alteraram de certa forma a função de contingências funcionalmente semelhantes apresentadas fora do contexto de terapia? Ou auxiliaram as clientes a ficarem sob controle de outras propriedades dos estímulos presentes? Ou seja, o fato das contingências estarem mais claras, sendo mais facilmente discriminadas, facilitou o reconhecimento de contingências semelhantes em outros contextos, promovendo as mudanças?

Tais perguntas continuam abertas, mas os dados indicam que ao menos no atendimento das clientes aqui analisadas, a parte experiencial da FAP mostrou-se suficiente para produzir tais mudanças, e a parte analítica talvez seja uma suplementação, ao menos em alguns casos, 
mas não necessária às mudanças esperadas. Permanece portanto a hipótese de que a combinação entre as partes experiencial e analítica da FAP pode aumentar a força da interação, levando a maiores resultados externos, mas não se confirma a hipótese de que tal combinação seja necessária.

Como discutido por Abreu-Rodrigues e Sanabio-Heck (2004), há que se considerar 1) que novas respostas podem ocorrer por um processo de modelagem na ausência de um controle verbal, 2) que regras podem reduzir a sensibilidade às contingências e 3) que regras em geral não descrevem as sutilezas dos contextos que podem ser importantes no responder. Assim, a ênfase que muitas vezes é dada ao uso de regras, pode ser substituída por uma ênfase no próprio processo de modelagem, como previsto pelos autores da FAP (Kohlenberg \& Tsai, 1991).

Em outras palavras, parece que para Dora e Sol foi mais importante viver a relação terapêutica, do que entendê-la.

\section{Outras possibilidades de contribuição das análises}

Apesar dos dados indicados na presente pesquisa não se mostrarem animadores quanto à suplementação que a parte analítica da FAP pode exercer sobre a parte experiencial, é possível que, se outros aspectos da Regra 5, CCR3 e O3 forem enfatizados em sessão, algum efeito mais evidente possa ser observado.

Nesse caso pode-se questionar se as regras teriam levado a um maior impacto nas melhoras das clientes se fossem realizadas em maior frequência (desde que não prejudicassem a parte experiencial da sessão). Poderia ser investigado ainda os efeitos das análises serem ainda mais focadas nas melhoras produzidas na relação terapêutica e em paralelos dessa com o ambiente externo do que o foram na presente pesquisa. Aqui, esses aspectos da Regra 5 foram investigados apenas de forma descritiva e não de forma experimental e se tais variáveis forem possíveis de serem isoladas, talvez possam ser observadas com um maior impacto do que o que foi aqui.

Outro fator possível de ser investigado e que não foi analisado no presente trabalho é quantos e quais os termos da contingência envolvidos nas análises realizadas por terapeuta e cliente. Análises descritivas sobre isso poderiam ser realizadas ou pesquisas experimentais poderiam ser conduzidas a fim de se identificar se análises que priorizem a especificação da consequência poderiam ter um maior efeito nas melhoras das clientes do que as que priorizam a especificação apenas do antecedente, conforme discutido na Introdução do presente trabalho. 
Pode-se também ser investigado se as análises emitidas descreviam o comportamento das clientes de forma mais funcional ou topográfica.

Ainda, como discutido por Kameyama (2012), diversos trabalhos que analisam em algum grau o comportamento de interpretação do terapeuta emitido em sessão evidenciam e discutem que tais respostas apresentam habitualmente longas falas e que uma análise dos mesmos apenas por percentual de ocorrência pode subestimar sua apresentação e portanto análises sobre sua duração podem ser importantes (Oshiro, 2011; Sadi, 2011; Zamigani, 2007). Tal análise de duração não foi realizada no presente trabalho.

Além disso, há ainda um outro aspecto da Regra 5 que foi propositalmente deixado de lado no presente trabalho, que é a solicitação direta de tarefas de casa, explicitamente introduzida na descrição da FAP em Tsai et al. (2009). Os autores discutem a importância do terapeuta sugerir diretamente que os ganhos atingidos e analisados em sessão sejam emitidos fora do contexto terapêutico. Nesse caso, o terapeuta estaria dando uma orientação direta, aumentando a chance de mudanças em outros contextos.

Os dados preliminares da presente pesquisa foram apresentados previamente em um congresso internacional (Villas-Boas, Meyer, \& Kanter, 2014) e algumas discussões interessantes foram levantadas. Entre elas, a Dra. Tsai (comunicação pessoal) observou que talvez se tivessem sido realizadas as solicitações de tarefas de casa, mais melhoras externas poderiam ter sido observadas, inclusive mais diretamente relacionadas com as Fases BCs, justificando que em sua experiência clínica, ela observa que tais solicitações produzem diferença no comportamento externo dos clientes.

É bastante provável que Dra. Tsai tenha razão em sua observação, até mesmo pelos dados apresentados na literatura sobre o tema. Donadone e Meyer (2005), por exemplo, realizaram uma pesquisa descritiva, comparando sessões de terapeutas comportamentais experientes e pouco experientes quanto a diferenças na emissão de orientações por parte do terapeuta e de auto orientações por parte dos clientes. Pela descrição que as autoras fizeram sobre as orientações e auto orientações analisadas, presume-se que elas se equivalem, ao menos em parte, às solicitações de tarefas de casa, descritas pela FAP ${ }^{22}$. As autoras observaram que, tanto terapeutas experientes como os pouco experientes, emitem regras em sessão voltadas para como os clientes poderiam agir em sua vida, principalmente orientações que especificam a

\footnotetext{
22 Nas palavras de Meyer (2009): “recomendação contempla verbalizações nas quais o terapeuta sugere alternativas de ação ao cliente ou solicita o seu engajamento em ações ou tarefas. Deve ser utilizada quando o terapeuta especifica a resposta a ser (ou não) emitida pelo cliente. A literatura refere-se a essa categoria também como aconselhamento, orientação, comando, ordem."
} 
topografia da ação. A partir dos dados supôs-se que orientações de forma geral estejam relacionadas com mudanças comportamentais fora da terapia. Meyer (2009) ampliou essa análise, verificando o uso de recomendações em sessões diversas de 88 terapias comportamentais conduzidas e constatou que terapeutas mais experientes emitiram mais recomendações do que terapeutas menos experientes. Além disso, a partir da análise de um outro conjunto de sessões, a autora verificou também que terapeutas de diferentes abordagens emitem orientações, o que indica ser esse um importante mecanismo de mudança terapêutica.

Donadone (2009) realizou ainda análises mais detalhadas das sessões investigadas em Donadone e Meyer (2005). Assim, Donadone (2009) ${ }^{23}$ verificou que emissões de orientações (tanto realizadas pelo terapeuta como pelo cliente) foram antecedidas por solicitações de reflexão (solicitar análises) e interpretação (fazer análises) por parte do terapeuta e principalmente por estabelecimento de relações por parte dos clientes. Além disso, observouse que os clientes de terapeutas experientes emitiam mais estabelecimento de relações antes de emitirem auto orientações do que os clientes de terapeutas menos experientes. Donadone (2009) concluiu que parece ser uma forma experiente de atuar terapeuticamente, evocando análises por parte dos clientes e em seguida modelando um repertório de auto orientação.

Como apresentado anteriormente, Mendes (2014) observou que na Fase BC1 realizada com Sol, a forma mais efetiva de produzir análises no comportamento da cliente foi através de evocações compostas por solicitações de reflexão; sendo a categoria interpretação também bastante frequente como formas de consequenciar análises e dar exemplo delas. Esses dados são condizentes com os dados de Donadone (2009) e pode-se supor que poderia ser efetiva a produção de orientações ou auto orientações (elaboração de tarefas de casa) a partir das análises realizadas com Sol, o que fortalece a hipótese da Dra. Tsai.

O que está sendo dito é que talvez, uma análise funcional detalhada sobre as melhoras ocorridas dentro de sessão, com a emissão de paralelos para a vida diária do cliente pode servir como uma boa base para solicitações de tarefas de casa específicas, talvez aumentando as chances de tais tarefas serem seguidas. Nesse caso, as análises serviriam como uma suplementação para a solicitação da tarefa de casa em si. Novamente, pelos dados indicados de melhoras extra sessão, supõe-se que a parte experiencial da FAP é suficiente para que ocorrem melhoras fora da sessão, mas talvez tais melhoras possam ser ainda maiores com a adição de tarefas de casa suplementadas pelas análises realizadas. Mais investigações são necessárias para se confirmar tal hipótese.

\footnotetext{
${ }^{23}$ Tais análises também podem ser lidas em Meyer (2009).
} 


\section{F) Análise de concordância}

Faz-se ainda importante mencionar mais especificamente a concordância encontrada entre a pesquisadora e aferidoras em relação às categorias do sistema FAPRS.

Das 58 sessões categorizadas pela terapeuta-pesquisadora, 12 foram aferidas, divididas entre quatro aferidoras, tendo uma delas realizado categorizações independentes e as demais apenas verificações. Apesar de serem várias sessões aferidas, a forma como a concordância foi calculada não pode ser considerada ideal e cabe aqui mencionar alguns aspectos que tem envolvido o cálculo da concordância na literatura de pesquisas com FAPRS.

Busch et al. (2009), por exemplo narra ter feito um longo processo de treino a fim de se obter concordâncias aceitáveis e mesmo assim só as obtiveram quando calculadas em duplas, nas quais havia ao menos um terapeuta experiente presente. Oshiro et al. (2012) apresenta um alto índice Kappa, mas explica que tal índice foi obtido após a sessão ter sido categorizada e as categorias revisadas entre a terapeuta-pesquisadora e o aferidor e algumas vezes alteradas. A própria discrepância entre o que é um valor de índice Kappa considerado aceitável entre Callaghan et al. (2008) e Popovitz (2013) indica que há ainda questões a serem melhor investigadas na área quanto a concordâncias de categorias realizadas com o FAPRS.

Os trabalhos de Oshiro et al. (2012) bem como o de Geremias (2014), apontam ainda para problemas metodológicos envolvendo o próprio terapeuta como categorizador de suas sessões, tal como realizado no presente trabalho. Assim como discutido por Oshiro et al. (2012) as categorizações serem feitas pelo próprio terapeuta pode trazer benefícios já que provavelmente nenhum outro categorizador poderia discriminar tão bem a ocorrência de CCRs em sessão quanto o próprio terapeuta. Follette e Bonow (2009) chegam a comentar sobre ser necessários que os categorizadores do FAPRS façam algumas inferências sobre as funções a longo prazo do comportamento do terapeuta e que isso pode ser um problema na precisão dos dados; porém apontam que isso não impediu a identificação do responder contingente do terapeuta nas pesquisas de Busch et al. (2009) e de Callaghan et al. (2003), que haviam sido publicados até então. O fato do terapeuta ter mais conhecimento a respeito dos CCRs do cliente e das funções de seu próprio comportamento, pode, no entanto, ser reconhecidamente um viés, que foi controlado, por exemplo, na pesquisa de Geremias (2014), que contou com um terapeuta conduzindo a terapia, que não a própria pesquisadora e principal categorizadora.

Acredita-se que na presente pesquisa maiores treinos com as aferidoras poderiam elevar os índices de concordância, ao habilitá-las para uma melhor discriminação dos CCRs e emissões de regras da terapeuta ocorrendo em sessão (tal aspecto foi também discutido por 
Kameyama, 2012). Por terem sido escolhidos CCRs sutis, e não óbvios, que correspondessem a um trabalho clínico feito em contexto natural (diferentemente do que foi descrito por Landes et al., 2013), tal treino talvez tenha sido dificultado (ver Danna e Matos, 1999; Kameyama, 2012; Meyer, 2009; Zamignani, 2007 para discussões sobre definições de categorias). Muitas horas foram gastas na presente pesquisa com a conceituação e definição dos CCRs e mesmo assim, não foi suficiente para se obter clareza de tais CCRs por observadores. Na época da realização das definições acerca da conceituação do caso, a terapeuta-pesquisadora utilizou o questionário FIAT para o terapeuta (Callaghan, 2006) como forma auxiliar para reflexão do caso, respondendo o instrumento referente ao terapeuta. Mas a definição das respostas que foram observadas (dentro e fora de sessão) não se baseou no instrumento para serem descritas, ao contrário do realizado por Kanter et al. (2006) e Lizarazo et al. (no prelo). Acredita-se agora que o uso de tal instrumento pudesse ter trazido uma maior clareza na descrição das classes de respostas selecionadas como alvo que poderia auxiliar no treino de concordância (ou até mesmo o registro externo das clientes). Porém, o fato do instrumento não ser traduzido ainda para a língua portuguesa dificulta que o mesmo possa ser utilizado diretamente com os clientes e espera-se que tal obstáculo seja superado em breve. Vale ainda ressaltar que, recentemente, Darrow, Callaghan, Bonow, \& Follette (2014) publicaram um estudo, no qual iniciou-se investigações sobre as propriedades psicométricas e estruturais do questionário de auto aplicação FIAT, que pode ser utilizado como uma forma de medida externa, a ser preenchida pelo próprio cliente.

De qualquer forma, parece no momento que, para uma boa concordância ser obtida, é necessário que se use classes de comportamento muito óbvias e não necessariamente as mais relevantes para o processo terapêutico (como mencionado por Landes et al., 2013, como uma limitação de sua pesquisa), ou então se requer que um longo treino sobre a conceituação de caso seja feito, o que pode ser por si só enviesante.

Outra possibilidade talvez, seja realizar a aferição em um momento mais inicial da pesquisa (e não ao final dela, como feito aqui), de modo que a categorização pelas aferidoras poderia influenciar e refinar o trabalho realizado pelo aferidor principal. Porém, também podese questionar se tal procedimento, apesar de parecer ser mais vantajoso em termos de pesquisa, também o seria em termos clínicos.

De qualquer modo, ficam abertas ainda as vantagens e desvantagens dessa forma de categorização e não cabe um aprofundamento dessa discussão no presente trabalho. Investigações maiores deveriam ser realizadas não apenas para se encontrar as melhores formas de se obter concordância entre pesquisadores (em especial quando o próprio terapeuta é um 
deles), mas também para se entender mais sobre o fenômeno da concordância que está sendo circunscrito aqui. Algumas perguntas podem ser levantadas: há vantagens e/ou desvantagens em o terapeuta ser o principal categorizador? Qual o momento adequado para que os aferidores iniciem seu treino para aferição? Esse treino deve influenciar na forma como o terapeuta categoriza suas próprias sessões ou até mesmo na forma como conduz suas sessões? Qual o nível de clareza de um CCR para ser identificado/discriminado pelos aferidores? Deve haver uma preocupação em identificar os CCRs a serem categorizados de acordo com o que está sendo trabalhado em sessão ou de acordo com o que é possível ser observador por outros (através de transcrição ou vídeo) que não o próprio terapeuta? O que seria mais importante em uma pesquisa clínica: obter a sutileza que o terapeuta é capaz de ter em sessão influenciando na análise dos dados ou a clareza e precisão necessárias para a replicação dos dados por aferidores externos? Muitas perguntas a respeito de aferições de concordância ficam, portanto, em aberto, e necessitam de maiores investigações futuras.

\section{G) Conclusões}

O objetivo do presente trabalho foi verificar se a Regra 5 da FAP é necessária ou ao menos auxiliar para que ocorram melhoras terapêuticas dentro e fora de sessão.

Há muito se questiona na literatura sobre qual forma de ação do terapeuta levaria a mudanças no dia a dia do cliente, especialmente pelo fato de serem necessárias mudanças nas contingências de vida dos clientes para que seus comportamentos mudem. Como o terapeuta não tem acesso diretamente às contingencias vividas no dia a dia pelos clientes, discute-se que o trabalho verbal feito em sessão acaba por ser responsável por essas mudanças (por exemplo, Abreu-Rodrigues \& Sanabio-Heck, 2004; Donadone, 2009; Kohlenberg et al., 1993; Meyer, 2000, 2004, 2009; Villas-Bôas et al., 2015; entre outros). Porém, questionou-se aqui se em uma terapia FAP, na qual prioriza-se a modelagem de repertório em sessão, a emissão de tais análises é de fato necessária.

Para isso analisou-se a terapia de duas clientes que foram submetidas a um delineamento de sujeito único com reversão (A-B-BC-B-BC), no qual $\mathrm{A}$ correspondeu a terapia comportamental sem uso sistemático da FAP, B a introdução das Regras 1 a 4 da FAP; e BC, a manutenção das regras previamente introduzidas, além da inclusão da Regra 5. Assim controlou-se a apresentação dessa última sendo possível verificar se ocorreriam mudanças no responder dos clientes em função de suas apresentações ou retiradas. 
Dados foram apresentados indicando que a terapia realizada, de fato seguiu o procedimento proposto na presente pesquisa. Além disso, considera-se que ambas as terapias foram bem sucedidas. Os dados de dentro da sessão indicam isso claramente. Os dados de fora da sessão são menos evidentes, mas tratados em conjunto, indicam também que houve melhoras no dia a dia de ambas as clientes. Uma certa piora, tanto dentro como fora de sessão foi observada para a cliente Sol nas últimas fases experimentais, mas atribui-se isso a mudança de foco da terapia, o que faz parte de um processo terapêutico, o qual acaba por passar por diferentes fases.

Foi observado um impacto no comportamento de análise realizadas em sessão pelas clientes, sendo que estas passaram a emitir mais análises, quando a terapeuta assim o fez. Verificou-se também que nas Fases BC passaram a ocorrer mais emissões de análises sobre melhoras das clientes que ocorreram em sessão tanto por parte da terapeuta como por parte das clientes. Foi verificado ainda que nessas fases a terapeuta priorizou evocações e modelagens de análises no responder das clientes ao invés de apenas apresentar a elas tais análises. Assim, parece que para se obter um aumento de comportamento de análise no repertório de clientes pode ser importante esforços em evocar e modelar análises e não apenas apresentar modelos dessas.

Por outro lado, na presente pesquisa, a emissão de análises não apresentou impacto sobre os CCR1s e CCR2s das clientes e tampouco nas melhoras e comportamentos problemas emitidos fora de sessão. Desse modo, mesmo que se tenha levantado formas de condução da terapia que aumentem emissões de análise por parte das clientes, não ficou claro qual seria a função dessas análises quando inseridas em uma terapia FAP.

É curioso perceber que cada uma das clientes apresentava um perfil diferente. Comparando-se as duas nota-se que Dora apresentou uma maior correlação entre as regras da FAP emitidas pela terapeuta e emissões de CCRs do que Sol. Já a cliente Sol, apresenta maior correlação do que Dora entre Regra 5 e emissões de análise realizadas por ela. Além disso, Dora descreveu-se como não estando disponíveis para análises ou comentários mais prolongados da terapeuta em boa parte do procedimento experimental, enquanto Sol sempre descreveu que análises a auxiliavam em suas melhoras. Tais dados indicam que Dora pode ser descrita como uma pessoa mais emocional, menos racional, ao contrário de Sol. Mesmo tendose clientes de diferentes perfis, foi observado que a Regra 5 não foi necessária para a produção de melhoras para nenhuma delas. O processo de terapia que parece ter sido importante nas melhoras acaba por ser o mesmo para as duas. Em outras palavras, parece que maior 
autoconhecimento não levou a maior autocontrole no caso dessas clientes, submetidas a um procedimento sistemático de modelagem de repertório, como previsto pela FAP.

Até onde se conhece esse foi o primeiro trabalho empírico e experimental que investigou aspectos e efeitos da Regra 5 da FAP. Há muito tempo hipóteses são levantadas a respeito da Regra 5 ter menos importância do que as Regras 2 e 3, ao mesmo tempo em que é tratada como sendo a responsável pela generalização das melhoras ocorridas em sessão para o dia a dia. Com o presente trabalho obtém-se um início de evidências que até o momento indicam ser a Regra 5 menos importante do que as responsáveis por evocar e modelar CCRs em sessão, bem como evidências iniciais de que essa regra não é essencial para a generalização dos ganhos ocorridos em terapia para fora da sessão terapêutica. Considerando-se, portanto, tal pesquisa como um grande avanço na área.

Alguns pontos positivos da presente pesquisa podem ser levantados. Por exemplo, acredita-se que a grande quantidade de sessões e consequentemente de falas de terapeuta e clientes analisadas aqui trouxeram peso aos dados observados. $\mathrm{O}$ fato de terem sido realizadas análises descritivas (microanálise a partir da subcategorização de respostas) em meio aos dados coletados experimentalmente, clareou os dados identificando mais diretamente as relações entre a Regra 5 e os comportamentos de análise emitidos pelas clientes.

Ainda, o fato de terem sido coletados dados de follow-up enriquecem ainda mais esses dados, ao se verificar a manutenção das melhoras obtidas em sessão e fora delas (pelas análises de Os, Episódios de Os e dados do EAS-40). Em especial, vale ressaltar que há grande predominância de CCR2s na sessão de follow-up para ambas as clientes. Isso ocorreu apesar do procedimento adotado se assemelhar às sessões da Fase $\mathrm{A}$, indicando que houve um aprendizado ao longo da terapia e do procedimento experimental que permitiu a emissão de melhoras mesmo que o ambiente terapêutico não estivesse voltado para suas evocações e modelagem, como ocorria nas fases FAP. Recomenda-se que novos estudos se utilizem de dados de follow-up, assim como feito também por Lizarazo et al. (no prelo).

Além do mais, as análises realizadas a partir das falas das clientes acerca dos Episódios de Os foram consideradas no presente trabalho como uma boa alternativa às análises de Os dentro de sessão, sendo mais recomendadas por serem mais fiéis ao que acontece fora de sessão terapêutica.

Por fim, considera-se como um ponto forte o fato de terem sido utilizados vários instrumentos de medidas que acabaram por se complementar e sugerir mais fortemente as melhoras ocorridas fora de sessão terapêutica. $O$ fato de não terem sido observadas diferenças entre fases com os dados coletados fora de sessão poderiam ser questionados levantando-se se 
tais diferenças de fato não existiram ou se os instrumentos não foram capazes de detectar. Como o único que indicou um dado nesse sentido foi o EAS-40 aplicado na cliente Dora, acredita-se aqui que possivelmente as melhoras não ocorreram de acordo com a introdução da VI (Regra 5), mas não descarta-se a hipótese de que pode haver questões metodológicas influenciando os dados aqui encontrados.

Quanto aos pontos fracos do presente trabalho, pode-se mencionar a forma como foi realizada a aferição de concordância das categorizações feitas a partir do FAPRS, levando às questões mencionadas anteriormente. Mais pesquisas à respeito mostram-se importantes para a área.

Outra limitação do presente estudo refere-se ao registro das clientes fora de sessão. Como discutido, algumas decisões metodológicas aqui realizadas possivelmente prejudicaram uma maior clareza desse dado. Em próximas pesquisas que visem a coleta de dados fora de sessão recomenda-se o uso de um terceiro pesquisador, que conheça a conceituação de caso, mas não as hipóteses da pesquisa, auxiliando o cliente no aprendizado do registro, sem que esse influencie diretamente na sessão terapêutica/experimental. Acredita-se também que períodos mais curtos de coleta de dados, ou que tenham períodos de pausas no registro externo, auxiliariam no engajamento e decorrente precisão do registro.

Além disso, algumas perguntas podem ser levantadas quanto ao procedimento aqui realizado e o quanto pode ter influenciado nos dados. Apesar das discussões já levantadas pergunta-se se uma quantidade maior de emissões de Regra 5 ou de CCR3 poderia ter tido um efeito maior nas melhoras externas (ou mesmo internas) das clientes; pergunta-se o que poderia ter sido feito para que tais emissões de análises por ambas as partes fossem maiores.

Pode-se questionar inclusive se, no presente estudo, as variáveis dependentes escolhidas foram as mais apropriadas para observar os efeitos da Regra 5. Talvez o impacto dessa recaia sobre algum outro comportamento que não foi aqui analisado. Pergunta-se, por exemplo, se as análises emitidas poderiam servir como uma boa base para solicitações de tarefas de casa, e assim aumentando os ganhos realizados fora de sessão terapêutica.

Ainda, será que o procedimento de reversão é o mais indicado para se observar melhoras externas ou o aparecimento de tais melhoras ocorre de forma tão mais lenta do que dentro da terapia requerendo um outro tipo de delineamento que torne os resultados mais evidentes?

Por fim, vêm se ampliando o número de evidências que confirmam a hipótese do principal mecanismo de ação da FAP e tem começado a surgir trabalhos que investigam processos paralelos a Regra 3, como esse aqui relatado. Alguns desses trabalhos estão em andamento com diversos objetivos relacionados a FAP como o de Vartanian (2015) que 
investiga mais a fundo seus mecanismos de ação, ao verificar os efeitos das Regras 2 e 3 em separados; ou estudos que pretendem investigar o uso da FAP com diferentes populações, como clientes com Transtorno de Estresse Pós-Traumático em casos de abuso sexual (Lima, 2015), clientes com Transtorno por Uso de Substâncias (Aranha, 2015), ou com crianças com Transtorno Desafiador Opositivo (Xavier, 2014). Espera-se que mais pesquisas continuem a fim de se aumentar o arcabouço de indícios a respeito da efetividade e mecanismos de ação da FAP, incluindo investigações a respeito dos demais aspectos da Regra 5 mencionados, mas não investigados aqui, como por exemplo, os efeitos das análises sobre elaborações de solicitações de tarefas de casa. 


\section{REFERÊNCIAS ${ }^{24}$}

Abreu, P. A., Hübner, M. M. C., \& Lucchese, F. (2012). The Role of Shaping the Client's Interpretations in Functional Analytic Psychotherapy, 28, 151-157.

Abreu-Rodrigues, J., \& Sanabio-Heck, E. T. (2004). Instruções e auto-instruções: contribuições da pesquisa básica. In: C. N. de Abreu \& H. J. Guilhardi. (Orgs.). Terapia comportamental e cognitivo-comportamental. (Vol. 1; pp. 152-168). São Paulo: Roca.

Andery, M. A. (2010). Métodos de pesquisa em análise do comportamento. Psicologia USP, 21(2), 313-342.

Aranha, A. S. (2015). Delineamento experimental de caso único: a Psicoterapia Analítica Funcional aplicada ao transtorno por uso de substâncias. Projeto de mestrado, Departamento de Psicologia Clínica, Instituto de Psicologia, Universidade de São Paulo, São Paulo.

Bedesem, P. L., \& Dieker, L. A. (2014). Self-monitoring with a twist: Using cell phones to CellF-Monitor On-Task Behavior. Journal of Positive Behavior Interventions, 16(4), 246254.

Bicard, D. F., \& Neef, N. A. (2002). Effects of Strategic versus tactical instructions on adaptation to changing contingencies in children with ADHD. Journal of Applied Behavior Analysis, 35, 375-389.

Bohm, C. H., \& Gimenes, L. S. (2008). Automonitoramento como técnica terapêutica e de avaliação comportamental, Revista Psicolog, 1(1), 88-100.

Bohm, C. H., \& Gimenes, L. S. (2012). Reatividade ao Automonitoramento em uma Portadora da Síndrome do Intestino Irritável. Psicologia: Teoria e Pesquisa, 28(3), 101-110.

Busch, A. M., Callaghan, G. M., Kanter, J. W., Baruch, D. E., \& Weeks, C. E. (2010). The Functional Analytic Psychotherapy Rating Scale: A replication and extension. Journal of Contemporary Psychotherapy, 40, 11-19. doi:10.1007/s10879-009-9122-8

Busch, A. M., Kanter, J. W., Callaghan, G. M., Baruch, D. E., Weeks, C. E., \& Berlin, K. S. (2009). A micro-process analysis of Functional Analytic Psychotherapy's mechanism of chance. Behavior Therapy, 40, 280-290.

Callaghan, G. M. (2006). The Functional Idiographic Assessment Template (FIAT) System. The Behavior Analyst Today, 7(3), 357-398.

Callaghan, G. M., \& Follete, W. C. (2008). FAPRS MANUAL: Manual for the Functional Analytic Psychotherapy Rating Scale. The Behavior Analyst Today, 9(1), 57-97.

\footnotetext{
${ }^{24}$ As referências do artigo da Introdução também estão sendo apresentadas aqui, a fim de facilitar possíveis consultas.
} 
Callaghan, G. M., Follette, W. C., Ruckstuhl Jr., L. E., \& Linnerooth, P. J. N. (2008). The Functional Analytic Psychotherapy Rating Scale (FAPRS): A Behavioral Psychotherapy Coding System. The Behavior Analyst Today, 9(1), 98-116.

Callaghan, G. M., Naugle, A. E., \& Follette, W. C. (1996). Useful constructions of the client therapist relationship. Psychotherapy, 33(3), 381-390.

Callaghan, G. M., Summers, C. J., \& Weidman, M. (2003). The Treatment of Histrionic and Narcissistic Personality Disorder Behaviors: A Single-Subject Demonstration of Clinical Improvement Using Functional Analytic Psychotherapy. Journal of Contemporary Psychotherapy, 33(4), 321-339.

Catania, A. C. (1998). Learning. Englewood Cliffs, NJ: Prentice-Hall.

Cerrutti, D. T. (1991). Discriminative versus reinforcing properties of schedules as determinants of schedule insensivity in humans. The Psychological Record, 41, 51-67.

Danna, M. F. \& Matos, M. A. (1999). Ensinando observação: uma introdução. São Paulo: Edicon.

Darrow, S. M., Callaghan, G. M., Bonow, J. T., \& Follette, W. C. (2014). The Functional Idiographic Assessment Template-Questionnaire (FIAT-Q): Initial psychometric properties. Journal of Contextual Behavioral Science 3, 124-135

Del Prette, G. (2011). Objetivos analítico-comportamentais e estratégias de intervenção nas interações com a criança em sessões de duas renomadas terapeutas infantis (Tese de Doutorado). Instituto de Psicologia, Universidade de São Paulo, São Paulo.

Donadone, J. C. (2009). Análise de contingências de orientações e auto-orientações em intervenções clínicas comportamentais (Tese de Doutorado). Instituto de Psicologia, Universidade de São Paulo, São Paulo.

Donadone, J. C., \& Meyer, S. B. (2005). Orientação e Auto-orientação em Atendimentos de Terapeutas Analítico-comportamentais Experientes e Pouco Experientes. Revista Brasileira de Terapia Comportamental e Cognitiva, VII(2), 219-229.

Fernández-Parra, A., \& Ferro-García, R. (2006). Psicoterapia analítico- funcional: una aproximación contextual funcional al tratamiento psicológico. EduPsykhé, 5(2), 203-229.

Ferro, G. R. (2008). Recent Studies in Functional Analytic Psychotherapy. International Journal of Behavioral Consultation and Therapy, 4(2), 239-249.

Ferro, G. R., \& Valero, A. L. (2015). Avances en la Psicoterapia Analítica Funcional. Avances en Psicología Latinoamericana, 33(1), 15-30. doi: dx.doi.org/10.12804/apl33.01.2015.02

Follette, W. C., \& Bonow, J. T. (2009). The Challenge of Understanding Process in Clinical Behavior Analysis: The Case of Functional Analytic Psychotherapy. The Behavior Analyst, $32,135-148$. 
Follette, W. C., Naugle, A. E., \& Callaghan, G. M. (1996). A Radical Behavioral Understanding of the Therapeutic Relationship in Effecting Change. Behavior Therapy, 27, 623-641.

Foster, S. L., Laverty-Finch, C., Gizzo, D. P., \& Osantowski, J. (1999). Practical Issues in SelfObservation. Psychological Assessment, 11(4), 426-438.

Garfield, S. L. (1995). Psychotherapy: An ecletic-integrative approach. New York: John Wiley e Sons.

Geremias, M. C. G. (2014). Manejo de esquivas emocionais na Psicoterapia Analítica Funcional: delineamento experimental de caso único (Dissertação de Mestrado). Instituto de Psicologia, Universidade de São Paulo, São Paulo.

Guilhardi, H. J. (1997). Com que contingências o terapeuta trabalha em sua atuação clínica. In: R. A. Banaco (Org.). Sobre Comportamento e Cognição (Vol. 1, pp. 322-337). São Paulo: ARBytes Editora.

Hatfield, D. R., \& Ogles, B. M. (2004). The Use of Outcome Measures by Psychologists in Clinical Practice. Professional Psychology: Research and Practice, 35(5), 485-491.

Hayes, S. (1981). Single case Experimental design and empirical clinical practice. Journal of Consulting and Clinical Psychology, 49(2), 193-211.

Hayes, S. C., Barnes-Holmes, D., \& Roche, B. (2001). Relational Frame Theory: A PostSkinnerian Account of Human Language and Cognition. NY: Kluwer Academic/PlenumPress.

Hayes, S. C., Kohlenberg, B. S., \& Melancon, S. M. (1989). Avoiding and altering rule-control as a strategy of clinical intervention. In: S. C. Hayes (Ed.), Rule-governed behavior: Cognition, contingencies, and instructional control (pp. 359-385). NY: Plenum Press.

Hayes, S. C., Zettle, R. D., \& Rosenfarb, R. (1989). Rule-following. In: S. C. Hayes (Ed.), Rule-governed behavior: Cognition, contingencies, and instructional control (pp. 191-220). NY: Plenum Press.

Hopko, D. R., \& Hopko, S. D. (1999). What can Functional Analytic Psychotherapy contribute to empirically-validated treatments. Clinical Psychology and Psychotherapy, 6, 349-356.

Jackson, J. L. (1999). Psychometric considerations in self-monitoring assessment. Psychological Assessment, 11(4), 439-447.

Johnston, J. M. (1979). On the relation between generalization and generality. Behavior Analyst, 2(2), 1-6.

Kameyama, M. (2012). Intervenções sobre comportamentos de clientes que produzem sentimentos negatives no terapeuta (Dissertação de Mestrado). Departamento de Psicologia Clínica, Instituto de Psicologia, Universidade de São Paulo, São Paulo. 
Kanter, J. W., Landes, S. J., Busch, A. M., Rusch, L. C., Brown, K. R., Baruch, D. E., \& Holman, G. (2006). The effect of contingent reinforcement on target variables in outpatient psychotherapy for depression: A successful and unsuccessful case using functional analytic psychotherapy. Journal of Applied Behavior Analysis, 39, 463-467.

Kazdin, A. E. (2002). Research design in clinical psychology (4a ed.). Boston, MA: Allyn \& Bacon.

Kazdin, A. E. (2008). Evidence-based treatment and practice: New Opportunities to Bridge Clinical Research and Practice, Enhance the Knowledge Base, and Improve Patient Care. American Psychologist, 63(3), 146-159.

Korotitsch, W. J., \& Nelson-Gray, R. O. (1999). An Overview of Self-Monitoring Research in Assessment and Treatment. Psychological Assessment, 11(4), 415-425.

Kohlenberg, R. J., \& Tsai, M. (1991). Functional analytic psychotherapy: A guide for creating intense and curative therapeutic relationships. NY: Plenum.

Kohlenberg, R. J., Tsai, M., Parker, C. R., Bolling, M. Y., \& Kanter, J. W. (1999). Focusing on the Client-Therapist Interaction - Functional Analytic Psychotherapy: A Behavioral Approach. European Psychotherapy, 1(1), 15-25.

Kohlenberg, R. J., Tsai, M., \& Dougher, M. J. (1993). The dimensions of clinical behavior analysis. The Behavior Analyst, 16, 271-282.

Kratochwill, T. R., Hitchcock, J. H. Horner, R. H. Levin, J. R., Odom, S. L., Rindskopf, D. M., \& Shadish, W. R. (2013). Single-Case Intervention Research Design Standards. Remedial and Special Education, 34(1), 26-38. doi: 10.1177/0741932512452794

Laloni, D. T. (2001). SCL-90-R: Adaptação, Precisão e Validade (Tese de Doutorado). PUC Campinas, Campinas.

Lambert, M. J., \& Hawkins, E. J. (2004). Measuring Outcome in Professional Practice: Considerations in Selecting and Using Brief Outcome Instruments. Professional Psychology: Research and Practice, 35(5), 492-499.

Landes, S. J., Kanter, J. W., Weeks, C. E., \& Busch, A. M. (2013). The impact of the active components of functional analytic psychotherapy on idiographic target behaviors. Journal of Contextual Behavioral Science, 2, 49-57.

Lima, G. O. (2015). A Psicoterapia Analítica Funcional como tratamento de transtorno de estresse pós-traumático (Projeto de Mestrado). Departamento de Psicologia Clínica, Instituto de Psicologia, Universidade de São Paulo, São Paulo.

Lizarazo, N. E., Muñoz-Martínez, A. M., Santos, M. M., \& Kanter, J. K. (no prelo). A Within Subjects Evaluation of the Effects of Functional Analytic Psychotherapy on In-Session and Out-of-Session Client Behavior. Psychological Record. 
Maitland, D. W., \& Gaynor, S. T. (2012). The Distinctiveness, Intimacy, and Efficacy of FAP Compared to Non-Directive Support: An Alternating Treatments Design Investigation (Master Thesis Project). Western Michigan University, Kalamazoo.

Maitland, D. W. M., \& Gaynor, S. T. (2012). Promoting Efficacy Research on Functional Analytic Psychotherapy. International Journal of Behavioral Consultation and Therapy, 7(2-3), 63-71.

Mangabeira, V. (2014). Efeitos da sinalização de intervenções na psicoterapia analítica funcional (Tese de Doutorado). Instituto de Psicologia, Universidade de São Paulo, São Paulo.

Mangabeira, V., Kanter, J. W., \& Del Prette, G. (2012). Functional Analytic Psychotherapy (FAP): A review of publications from 1990 to 2010. International Journal of Behavioral Consultation and Therapy, 7(2-3), 78-89.

Matos, M. A. (1990). Controle experimental e controle estatístico: a filosofia do caso único na pesquisa comportamental. Ciência e Cultura, 42(8), 585-592.

Matos, M. A. (2001). Comportamento governado por regras. Revista Brasileira de Terapia Comportamental e Cognitiva, 3(2), 51-66.

Mendes, E. B. (2014). Relação entre interpretação ou solicitação de reflexão pelo terapeuta e comportamentos de análise por parte do cliente (Relatório de Iniciação Científica). Instituto de Psicologia, Universidade de São Paulo, São Paulo.

Meurer. P. H. (2011). Efeito da apresentação de feedback no comportamento do terapeuta de evocar e responder aos comportamentos clinicamente relevantes (Dissertação de Mestrado). Universidade Federal do Paraná, Curitiba.

Meyer, S. B. (2000). Mudamos, em terapia verbal, o controle de estímulos?. Acta Comportamentalia, 8(2), 215-225.

Meyer, S. B. (2004). Processos comportamentais na psicoterapia. In: Ciência do Comportamento. Conhecer e Avançar (Vol. 4, pp. 151-157). Santo André: ESETec.

Meyer, S. B. (2005). Regras e auto-regras no laboratório e na clínica. In: J. Abreu-Rodrigues \& M. R. Ribeiro (Orgs.), Análise do Comportamento: pesquisa, teoria e aplicação (pp. 211227). Porto Alegre: ARTMED Editora S.A.

Meyer, S. B. (2009). Análise de 'solicitação de informação' e 'recomendação' em banco de dados de terapias comportamentais (Tese de Livre Docência). Departamento de Psicologia Clínica, Instituto de Psicologia, Universidade de São Paulo, São Paulo.

Michael, J. (1982). Distinguishing between Discriminative and Motivational Functions of Stimuli. Journal of the Experimental Analysis of Behavior, 37(1), 149-155. 
Michael, J. (1993). Establishing Operations. Journal of the Experimental Analysis of Behavior, 16(2), 191-206.

Oshiro, C. K. B. (2011). Delineamento experimental de caso único: a Psicoterapia Analítica Funcional com dois clientes difíceis (Tese de Doutorado). Departamento de Psicologia Clínica, Instituto de Psicologia, Universidade de São Paulo, São Paulo.

Oshiro, C. K. B., Kanter, J. W., Meyer, S. B. (2012). A Single-Case Experimental Demonstration of Functional Analytic Psychotherapy with Two Clients with Severe Interpersonal Problems. International Journal of Behavioral Consultation and Therapy, 7(2-3), 111-116.

Pankey, J. (2012). Functional Analytic Psychotherapy (FAP) for Cluster B Personality Disorders: Creating Meaning, Mattering, and Skills. International Journal of Behavioral Consultation and Therapy, 7(2-3), 117-124.

Popovitz, J. M. B. (2013). A resposta contingente do terapeuta aos comportamentos clinicamente relevantes: especificação e avaliação (Dissertação de Mestrado). Universidade Federal do Paraná, Curitiba.

Pryor, K. (1984). Don't Shoot the Dog. NY: Bantam.

Sadi, H. M. (2011). Análise dos comportamentos de terapeuta e cliente em um caso de Transtorno de Personalidade Borderline (Tese de Doutorado). Departamento de Psicologia Clínica, Instituto de Psicologia, Universidade de São Paulo, São Paulo.

Sampaio, A. A. S., Azevedo, F. H. B., Cardoso, L. R. D., Lima, C., Pereira, M. B. R., \& Andery, M. A. B. A. (2008). Uma introdução aos delineamentos experimentais de sujeito único. Interação em Psicologia, 12(1), 151-164.

Schlinger, H., \& Blakely, E. (1987) Function-Altering Effects of Contingency-Specifying Stimuli. The Behavior Analyst, 10(1), 41-45.

Schlosser, R. W. (2004). Goal attainment scaling as a clinical measurement technique in communication disorders: A critical review. Journal of Communication Disorders, 37, 217 239.

Sidman, M. (1960). Tactics of scientific research: evaluating experimental data in psychology. New York: Basic Books, Inc., Publishers.

Siegel S., \& Castellan N. (1988). Nonparametric Statistics for the Behavioral Sciences (2 $\left.{ }^{\mathrm{a}} \mathrm{Ed}\right)$. New York: McGraw-Hill.

Simões Filho, E. F. (2014). Manejo de metáforas em psicoterapia analítico-comportamental (Dissertação de Mestrado). Departamento de Psicologia Clínica, Instituto de Psicologia, Universidade de São Paulo, São Paulo. 
Smith, J. D. (2012). Single-Case Experimental Designs: A Systematic Review of Published Research and Current Standards. Psychological Methods, 17(4), 510-550. doi: $10.1037 / \mathrm{a} 0029312$

Skinner, B. F. (1953). Science and Human Behavior. NY: Macmillan Company.

Skinner, B. F. (1957). Verbal behavior. New Jersey: Prentice-Hall.

Skinner, B. F. (1966). An operant analysis of problem solving. In B Kleinmuntz (Ed.) Problem solving: Research, method and theory (pp. 133-171). New York: John Wiley \& Sons.

Skinner, B. F. (1969). Contingencies of Reinforcement: A Theoretical Analysis. NY: Meredith Corporation.

Skinner, B. F. (1974). About behaviorism. NY: Vintage books.

Skinner, B. F. (1989). Recent issues in the analysis of behavior. Columbus: Merril Publishing Company.

Starlin, R. R. (2010). Prática controlada: medidas continuadas e produção de evidências empíricas em terapias analítico-comportamentais (Tese de Doutorado). Departamento de Psicologia Clínica, Instituto de Psicologia, Universidade de São Paulo, São Paulo.

Törneke, N., Luciano, C., \& Valdivia Salas, S. (2008). Rule-governed behavior and psychological problems. International Journal of Psychology and Psychological Therapy, $8(2), 141-156$.

Tourinho, E. Z. (2006). O autoconhecimento na Psicologia Comportamental de B.F. Skinner. Santo André: ESETec Editores Associados.

Tsai, M., Kohlenberg, R. J., Kanter, J. W., Holman, G. I., \& Loudon, M. P. (2012). Functional Analytic Psychotherapy. Cornwall: TJ International Ltd.

Tsai, M., Kohlenberg, R. J., Kanter, J. W., Kohlenberg, B., Follette, W. C., \& Callaghan, G. M. (2009). A guide to Functional Analytic Psychotherapy: Awareness, courage, love and behaviorism. New York: Spring.

Vartanian, J. F. (2015). A evocação de comportamentos clinicamente relevantes na psicoterapia analítica funcional: um delineamento experimental de caso único (Projeto de Mestrado). Departamento de Psicologia Clínica, Instituto de Psicologia, Universidade de São Paulo, São Paulo.

Villas-Bôas, A., Meyer, S., \& Kanter, J. (2014). What Kind of Talk Matters in Functional Analytic Psychotherapy? A Single-Case Experimental Design. In ACBS World Conference 12 (Resumo disponível em http://contextualscience.org/wc12_symposia_detail), Minneapolis, MN. 
Villas-Bôas, A., Meyer, S., Kanter, J., Callaghan, G. (2015). The use of Analytic Interventions in Functional Analytic Psychotherapy. Behavior Analysis: Research and Practice, 15(1), 119.

Wechsler, A. M., \& Amaral, V. L. R. (2009). Correspondência verbal: uma revisão da literatura. Revista Brasileira de Terapia Comportamental e Cognitiva, 11(2), 189-208.

Weeks, C. E., Kanter, J. W., Bonow, J. D., Landes, S. J., \& Busch, A. M. (2012). Translating the Theoretical Into Practical: A Logical Framework of Functional Analytic Psychotherapy Interactions for Research, Training and Clinical Purposes. Behavior Modification, 36(1), 87-119. doi: 10.1177/0145445511422830

Wetterneck, C. T., \& Hart, J. M. (2012). Intimacy is a Transdiagnostic Problem for Cognitive Behavior Therapy: Functional Analytical Psychotherapy is a solution. International Journal of Behavioral Consultation and Therapy, 7(2-3), 167-176.

Xavier, R. N., Kanter, J. W., \& Meyer, S. B. (2012). Transitional Probability Analysis of Two Child Behavior Analytic Therapy Cases. International Journal of Behavioral Consultation and Therapy, 7(2-3), 182-188.

Xavier, R. N. (2014). A Psicoterapia Analítica Funcional com crianças com transtornos desafiador e de oposição (Projeto de Doutorado). Departamento de Psicologia Clínica, Instituto de Psicologia, Universidade de São Paulo, São Paulo.

Yoshida, E. M. P., \& Silva, F. R. C. S. (2007). Escala de Avaliação de Sintomas-40 (EAS-40): validade e precisão em amostra não-clínica. Revista Semestral da Associação Brasileira de Psicologia Escolar e Educacional (ABRAPEE), 11(1), 89-99.

Zamignani, D. R. (2007). O desenvolvimento de um sistema multidimensional para a categorização e análise de comportamentos na interação terapeuta-cliente (Tese de Doutorado). Universidade de São Paulo, São Paulo. 
APÊNDICE A

FOLHA DE REGISTRO EXTERNO UTILIZADA COM A CLIENTE DORA 


\section{Cliente Dora}

\begin{tabular}{|l|c|c|c|c|c|c|c|}
\hline $\begin{array}{l}\text { Você, sentindo emoção em situações com } \\
\text { outras pessoas, na última semana }\end{array}$ & $\begin{array}{c}02 / 05 \\
\text { Sex }\end{array}$ & $\begin{array}{c}03 / 05 \\
\text { Sab }\end{array}$ & $\begin{array}{c}04 / 05 \\
\text { Dom }\end{array}$ & $\begin{array}{c}05 / 05 \\
\text { Seg }\end{array}$ & $\begin{array}{c}06 / 05 \\
\text { Ter }\end{array}$ & $\begin{array}{c}07 / 05 \\
\text { Qua }\end{array}$ & $\begin{array}{c}08 / 05 \\
\text { Qui }\end{array}$ \\
\hline Estourar & & & & & & & \\
\hline Usou válvula de escape & & & & & & & \\
\hline Pensamentos desorganizados & & & & & & & \\
\hline
\end{tabular}


APÊNDICE B

FOLHA DE REGISTRO UTILIZADA COM OBSERVADOR EXTERNO DA CLIENTE DORA 
Favor preencher o quanto antes e enviar-nos pelo correio

Data:

Sua amiga, nos últimos 2 meses, sentindo emoção em situações com outras pessoas:

1) Estourou

$\begin{array}{ccccc}\text { Raramente } & \text { Poucas vezes } & \text { Algumas vezes } & \text { Muitas vezes } & \text { Com frequência } \\ \square & \square & \square & \square & \square\end{array}$

2) Não se importou

\begin{tabular}{|c|c|c|c|c|}
\hline $\begin{array}{l}\text { Raramente } \\
\quad \square\end{array}$ & $\begin{array}{l}\text { Poucas vezes } \\
\square\end{array}$ & $\begin{array}{l}\text { Algumas vezes } \\
\square\end{array}$ & $\begin{array}{l}\text { Muitas vezes } \\
\square\end{array}$ & $\begin{array}{l}\text { Com frequência } \\
\square\end{array}$ \\
\hline \multicolumn{5}{|l|}{ 3) Se calou } \\
\hline Raramente & Poucas vezes & Algumas vezes & Muitas vezes & Com frequência \\
\hline$\square$ & $\square$ & $\square$ & $\square$ & $\square$ \\
\hline \multicolumn{5}{|c|}{$\begin{array}{l}\text { 5) Foi assertiva (teve coragem de falar/fazer o que queria, na relação com o outro, sem violar } \\
\text { os direitos do outro) }\end{array}$} \\
\hline Raramente & Poucas vezes & Algumas vezes & Muitas vezes & Com frequência \\
\hline$\square$ & $\square$ & $\square$ & $\square$ & $\square$ \\
\hline \multicolumn{5}{|c|}{ 6) Usou válvula de escape } \\
\hline Raramente & Poucas vezes & Algumas vezes & Muitas vezes & Com frequência \\
\hline$\square$ & $\square$ & $\square$ & $\square$ & $\square$ \\
\hline \multicolumn{5}{|c|}{ Fez coisas que teve vontade } \\
\hline Raramente & Poucas vezes & Algumas vezes & Muitas vezes & Com frequência \\
\hline$\square$ & $\square$ & $\square$ & $\square$ & $\square$ \\
\hline
\end{tabular}


APÊNDICE C

FOLHA DE REGISTRO EXTERNO UTILIZADA COM A CLIENTE SOL 


\section{Cliente Sol}

\begin{tabular}{|l|c|c|c|c|c|c|c|}
\hline & $\begin{array}{c}27 / 09 \\
\text { Sex }\end{array}$ & $\begin{array}{c}28 / 09 \\
\text { Sab }\end{array}$ & $\begin{array}{c}29 / 09 \\
\text { Dom }\end{array}$ & $\begin{array}{c}30 / 09 \\
\text { Seg }\end{array}$ & $\begin{array}{c}01 / 10 \\
\text { Ter }\end{array}$ & $\begin{array}{c}02 / 10 \\
\text { Qua }\end{array}$ & $\begin{array}{c}03 / 10 \\
\text { Qui }\end{array}$ \\
\hline Procurou por amigos, quando teve vontade & & & & & & & \\
\hline $\begin{array}{l}\text { Não procurou por amigos, mesmo tendo } \\
\text { vontade }\end{array}$ & & & & & & & \\
\hline Fez algo por você, ao invés de fazer pelo outro & & & & & & & \\
\hline $\begin{array}{l}\text { Fez algo pelo outro ao invés de fazer por você } \\
\text { Fez ou deixou de fazer algo para agradar o } \\
\text { outro }\end{array}$ & & & & & & & \\
\hline
\end{tabular}


APÊNDICE D

USO DAS REGRAS DA FAP COM CADA CLIENTE 


\section{Uso das regras com Dora}

\section{Categorias utilizadas para o comportamento do terapeuta}

Regra 1: Terapeuta avalia CCR1 ou CCR2, fazendo ou não um paralelo de fora para dentro da sessão.

$\mathrm{C}$ : Ai eu pude ver que realmente eu tenho uma ansiedade grande. - $C R B 2$

T: E agora você está se sentindo ansiosa? - Regra 1

C: Bastante. Tô fazendo acupuntura. Aí tem o ponto da ansiedade. Eu percebo que, eu fico bem, eu fico bem! - $C P R$

T: Então eu vou fazer mais uma pergunta. Se for ainda invasiva, ainda tendenciosa, você me fala. Mas o jeito que você me contou isso agora, você acha que é parecido com o jeito que você falaria com a sua tia se ela tivesse fazendo uma pergunta invasiva ou tendenciosa, qualquer coisa assim? - Regra 1

Regra 2: Terapeuta evoca, pede ou diretamente (verbalmente) solicita um CCR1 ou CCR2. Esse código NÃO depende do comportamento do cliente que o segue.

- A depender do contexto da sessão, elogios da terapeuta sobre atitudes da cliente ou comentários sobre suas dificuldades podem ser considerados como Regra 2 por evocarem CRBs do tipo B.

T: Eu imagino o quão difícil seja lidar com isso porque vem muito essa cobrança externa e você tá sempre se cobrando por isso. - Regra 2

T: Porque que é tão difícil imaginar ele (ex-marido) levando ela (filha) pra lá? - Regra 2

TRB1: Terapeuta responde efetivamente ao CCR1, incluindo a descrição de sentimentos do terapeuta em resposta ao cliente.

- Paráfrases em respostas a CRB1s são consideradas TRB1s, pois ajuda a cliente a elaborar a ideia ou lhe dá modelo.

C: E aí não precisa provocar. "Você táva (nervosa)? Você tá?" Porque ai você me provoca, ai já me provoca sentimentos mais... como posso dizer... primitivos, entendeu? Não é nem saudável isso. - CRB1

T: Pois é, mas quando eu te pergunto eu não tô tentando te provocar mais, aumentar, trazer esses sentimentos mais negativos, nada disso eu... - TRB1

C: Eu... "Fica nervosa! Fica nervosa! Fica nervosa!"... - CRB1

(T pede para $C$ preencher um instrumento de coleta de dados)

$\mathrm{C}$ : Ai tenho tanta coisa pra te falar, mas... -CRB1

T: Ai Jesus... não calma, esse daqui é rápido, enquanto eu pego o atestado, você preenche. $-T R B 1$

C: Deixa o atestado pra depois. $-C R B 1$

T: É que eu vou ter que pedir pra você preencher esse negócio antes da gente começar a sessão. - TRBI 
C: Não, ele não dirige. Nem carteira de habilitação ele tem. Mas ele é ótimo, ta? Ele é um bonzão! Ele não dirige porque ele não quer, porque ele é um incapaz, porque é um enrustido! Teve um problema na família dele lá, e ele ficou com trauma. Então, tem vários fatores que iam dificultar ele tirar essa carteira. - CRB1

T: Você tá com muita raiva dele, né? - TRB1

$\mathrm{C}$ : Eu acho que eu fiquei falando muito "Porque ele vai ver, porque ele vai, ah! Porque eu sou assim mesmo, quando eu pego uma briga, então ai eu não saio dela!”. Eu tenho que parar com isso. Eu desprendo toda a minha energia pra essa pouca coisa. Como se ela fosse uma coisa, mais absoluta do mundo. E não é! - CRB1

T: Pelo que eu tô entendendo você agora tá sentindo aquilo como falso. - TRBI

TRB2:Terapeuta responde efetivamente ao CCR2, incluindo a descrição de sentimentos do terapeuta em resposta ao cliente.

- Validações para CCR2, ou empatia, serão considerados TRB2

- Pedidos de continuidade da fala ou aprofundamento da mesma

- Atender aos pedidos dela quando feitos de forma assertiva

C: Até então eu falava assim "Eu não vou casar, eu vou cuidar dos meus pais". E quando eles decidiram ir embora, e foi ela, ficou muito forte pra mim. Eu chorava, pedia pra voltar, eles davam risada, achavam engraçado, achavam bonito, sei lá, entendeu? "Ai, como eu sou demais", né? - CRB2

T: Mas não entendiam o tamanho do sofrimento que você tava. - TRB2

C: Exatamente. Foi muito... E assim, eles usaram muito desse meu apelo emocional. CRB2

$\mathrm{T}:$ Como? - TRB2

C: Pra também assim, sabendo que eu tinha dependência deles, então para o... para a minha existência eles precisavam primeiro existir. Então eu também tinha que ajuda-los pra que eles continuassem existindo. $-C R B 2$

\section{(C perguntando se poderá ver a pesquisa/tese da $T$ )}

C: Uhum. Entendi. Mas de qualquer forma assim, ah, eu acho que... pra mim vai ser importante. Eu acho não, eu tenho certeza. - CRB2

T: Eu respeito muito isso, a clareza com que você traz isso, e a sua vontade de ver e justamente, eu vou te mostrar, e não tenho resistência nenhuma a isso. (...) - TRB2

C: Agora eu não tenho resposta pra nada, mas... eu te pergunto, você vai conversar? Você vai falar? - CRB2

T: Você está me pedindo pra falar, né? - TRB2

C: Estou pedindo pra você falar. $-C R B 2$

T: Então eu vou falar. Vou falar. E me pergunte de novo, porque pode ser que em outros assuntos acaba não surgindo deu te falar, mas me pergunte de novo que eu te conto. - TRB2 
Regra 5: Terapeuta discute (analisa/estabelece relações), evoca ou reforça (análises/estabelecimento de relações) CCR3, O3 ou paralelos entre os dois, buscando por generalização do comportamento do cliente.

C: É, pois é. Então, até que foi tranquilo na sexta-feira e tudo mais, eu me senti muito melhor quando terminou a sessão...-CTR

T: Você saiu melhor daqui? Porque eu fiquei me perguntando como você teria ficado ao longo da semana. - Regra 4

C: É, eu também fiquei me perguntando como será que eu ia ficar, mas eu fiquei muito melhor. Eu fui trabalhar bem, eu fiquei bem, foi tudo ótimo. - Pos-Rep

T: Você consegue entender o que que te fez bem? - Regra 5

$\mathrm{C}$ : Eu acho que assim, a possibilidade de falar e não brigar. Sabe... $-C R B 3$

T: Que gostoso ouvir essa resposta. Porque eu acho que isso foi o grande ganho de semana passada. - Regra 5

T: E pensando nessa sua fala, você colocou o limite no domingo. Você falou "Você não vai na aula", e ai veio essa resposta, ele (pai) não traz o (filho). E ai você ficou se corroendo não só no "como é que eu vou fazer no resto do dia" ou "como é que eu vou reagir dependendo do que ele fizer", mas "então o que eu fiz vai ter essa consequência". - Regra 5

T: Quer dizer, no fundo você tá vendo diferenças nele, e você tá com medo de gostar, e de baixar a guarda, porque você acha que tem certas coisas, como a mãe dele, como a relação dele com a mãe dele, que não vão mudar. - Regra 5

T: Eu acho que eles de certa forma entendem que você é nervosa. É uma característica sua. E eles entendem e respeitam porque sabem que você não é SÓ nervosa. Você tem momentos nervosa. - Regra 5

T: E como é que é, porque comigo você pode falar e com as pessoas não? - Regra 5 $\mathrm{C}$ : Ah, porque acho que você me conhece um pouco. Então, sei lá, se eu falar "Não tô a fim de responder" acho que você já entende um pouco e respeita. A (amiga) me conhece. E mesmo assim às vezes ela me insiste. Insiste em ficar de pergunta. $E$ às vezes eu falo "Ah, para! Faz do jeito que você quiser!". - CRB3

T: E você acha que você fala claramente como você fala pra mim? - Regra 5

$\mathrm{C}$ : Não, porque se ela insiste muito eu sou grossa. Eu falo "Ah, faz do jeito que você quiser!". - O3

T: Então, mas será que não é mais fácil falar claramente pra ela que nem você fala pra mim? - Regra 5

C: Mas eu falo! Mas ai a pessoa insiste! Ela ignora o que eu respondo. E ai ela insiste de novo. Ai na verdade, ela eu conheço bem, então muitas vezes ela me pergunta porque ela quer a minha resposta, mas não vai fazer diferença a minha resposta. - O3 T: Você falou já dessa sensação de falar "Faz A" "Ah, mas eu acho que eu vou fazer B" - Regra 5 
TO1: Terapeuta descreve ou comenta comportamento problema específico $(\mathrm{O} 1)$ do cliente que ocorre fora da sessão - diferente de analisar ou estabelecer relações.

T: Não sei se a gente já falou sobre isso nesses olhos, com esses olhos, mas... Você adora se doar. Não sei se é a Dora, mas você costuma fazer isso com frequência. E muitas das brigas que você compra é pelos outros. - TO1

C: Assim, as vezes eu surto de perder a razão... Não lembro mais o que eu falei, e eu não tenho um pingo de vergonha, nem de pudor, e que se dane se o papa tiver olhando, se tiver alguém da igreja não tô nem ai, e também vai praquele lugar e pronto. - O1 T: Você perde completamente a noção. - TO1

TO2: Terapeuta descreve ou comenta comportamento de melhora específico $(\mathrm{O} 2)$ do cliente que ocorre fora da sessão - diferente de analisar ou estabelecer relações.

T: Né? Pode ser que você possa vir a bater, mas olha o quão grosseiro ele foi, o quão autoritário ele foi, e o J. já bateu boca com ele. Mas você não bateu. - TO2

(C contando de provocação feita pelo ex-marido)

$\mathrm{C}$ : Eu acho que não fiquei (brava), nem disfarcei. Porque no fundo eu sabia que de fato ele não ia! - $O 2$

T: Você entendeu a provocação. - TO2

\section{Uso das regras com Sol}

\section{Categorias utilizadas para o comportamento do terapeuta}

Regra 1: Terapeuta avalia CCR1 ou CCR2, fazendo ou não um paralelo de fora para dentro da sessão.

T: Você tá triste agora? - Regra 1

T: E me falar isso é difícil? - Regra 1

T: Tá... E você acha que.. eu imagino o stress de toda essa situação do escritório e tudo o mais e falar sobre isso, será que também é mais uma forma de fazer pelos outros? Ou será que teriam outras coisas que você poderia estar falando que é mais fazer por você? ... - Regra 1

T: Posso fazer um desvio, mas ficando no mesmo assunto? Uma pergunta acho que difícil até talvez. Você acha que você evita esse tipo de acontecimento aqui comigo? Regra 1

C: Acho que não. $-C P R$

T: Tá. Você não está tentando ser uma cliente perfeita? - Regra 1

Regra 2: Terapeuta evoca, pede ou diretamente (verbalmente) solicita um CCR1 ou CCR2. Esse código NÃO depende do comportamento do cliente que o segue. 
- Perguntas sobre sentimentos, mesmo que no início da sessão (mas não ao final, para finalizar a sessão);

- Convites e condução de meditações e momentos de contato visual;

- Perguntas como: "o que você achou disso?", ou "o que você pensa ou sente em relação a isso?" "o que você sentiu?” são consideradas Regra 2 por estarem evocando CRBs dela falar dela mesma ou críticas em relação a abusos ou mesmo tolerância em relação a ela mesma ou aos outros. A depender do contexto, outras falas da terapeuta, como críticas a situações de abuso ou apontar incoerências dos demais, também podem ser consideradas Regra 2 por também terem esse efeito evocativo da cliente de reconhecer e criticar abusos que foram feitos a ela. (Essas mesmas falas, quando seguirem críticas feitas pela própria cliente, tem a função de reforçar as falas anteriores, sendo categorizadas então como TRB2);

- Falas sobre pedidos de planos, resoluções de problemas, são consideradas Regra 2.

T: Você falou, né, que você guardou demais. Como você acha que você pode se precaver pra não passar por isso de novo? É não explodindo como você explodiu ou é não guardando como você guardou? - Regra 2

T: Aham. E como você se sente de pelo menos nesse momento não estar conseguindo nem falar, nem deixar pra lá. Quão incômodo é isso? - Regra 2

\section{T: Como é que você tá? - Regra 2}

T: Você tava muito, acho que decidida mesmo, a sair da casa da sua tia né, assim que possível, você continua com essa ideia? - Regra 2

C: É, eu fico vendo se isso vai acontecer todo dia, né. Só que aí não respondi nada do que ela falou. E aí depois de uns trinta minutos, assim, que eu tava lá no meu quarto, quando eu passei por algum motivo, assim, perto dela, ela deve ter ficado pensando, né, porque eu ando deixando ela falando sozinha agora. Daí ela falou: ah, você me desculpa, eu não sabia que eu tinha que avisar. Também não respondi. - O1

T: O que você achou dessa desculpa dela? Foi realmente um pedido de desculpa ou...?

- Regra 2

TRB1: Terapeuta responde efetivamente ao CCR1, incluindo a descrição de sentimentos do terapeuta em resposta ao cliente.

T: É curioso porque tava tudo bem nebuloso pra você, né. E acho que essa tensão tava tomando conta. Mas ao mesmo tempo eu vi carinho no seu olho. $\mathrm{O}$ medo de estar nessa situação, acredito, mas também eu vi muito carinho, sabe. E o que eu tava sentindo, tentando te demonstrar era, nossa, que p.. admiração que tô, sabe. Tudo isso que você tá me contando de como você tá entendendo as coisas e enfrentando elas, é muita admiração. Era isso que eu tava tentando te demonstrar. Era isso que eu tava sentindo. $-T R B 2$ 
C: Eu acho que eu não consigo ter essa...Agir dessa forma. Até pela dificuldade que eu tenho de receber, ter algum elogio ou algum comentário sobre mim, tanto quanto eu tenho dificuldade de falar as coisas para as pessoas. - CRBI

T: Agora eu acabei de falar sobre a admiração que eu sinto, né. Como é que você se sentiu ouvindo da minha admiração? - TRB1

T: Tá. E você acha que você consegue ver acho que até de uma forma mais concreta mesmo o carinho, o afeto, o apreço, a admiração que eu sinto por você ou é só preocupação? - TRB2

$\mathrm{C}$ : Eu acho que sim. E aí também me faz pensar o quanto eu sou difícil de me aproximar das pessoas. $-C R B 1$

T: Por que? Como que uma coisa liga à outra? - TRB1

T: Você pensa nisso com frequência? Do fato de que ela não vai estar aí pra sempre? - Regra 2

C: Um pouco. $-C R B 1$

T: Você tem esse medo? - TRB1

$\mathrm{C}$ : Eu tenho porque principalmente pelos casos de doenças frequentes na família, né. Pelas pessoas que a gente perdeu. Acho que nos últimos seis anos. E ela foi fumante durante muitos anos. E eu sempre percebo, assim, que ela tem dores e tem cansaço e tudo. Eu vejo que ela não tem uma saúde boa, assim. Operou ainda no último ano. Operou vesícula, operou apêndice. Então não sei. Eu sempre penso nisso. - CRB1

T: Obrigada. Como é que você tá? - Regra 2

C: Bem. $-C R B 1$

T: Bem? - TRB1

C: Bem cansada. $-C R B 1$

T: Naquela correria de final de ano? - TRB1

T: Te dá raiva? - Regra 2

C: Não raiva. É uma tristeza assim muito grande e uma angústia de pensar que eu nunca vou conseguir fazer ela enxergar isso. $-C R B 1$

T: Será que não? - TRB1

$\mathrm{C}$ : Eu não sei mais como fazer. - $C R B 1$

C: Não totalmente, mas sempre fica assim... Eu sempre tenho aquela coisa de eu poderia ter feito melhor. - CRBI

T: Pois é. Eu fico pensando, né, essa sua tendência de sempre se exigir mais, né. - TRB1

TRB2:Terapeuta responde efetivamente ao CCR2, incluindo a descrição de sentimentos do terapeuta em resposta ao cliente.

- Respostas de empatia para expressão de sentimento ou para críticas aos demais e respostas de facilitação para o que está sendo dito, são consideradas TRB2

T: Tá. A vontade tá lá, né. E o que que te segura? - Regra 2 
C: Eu acho que vergonha também. - CRB2

$\mathrm{T}$ : Mais de mim do que dos outros. - TRB2

C: Acho que sim. $-C R B 2$

T: Você consegue reconhecer porquê? É, porque é o oposto do falar, né. - TRB2

$\mathrm{C}$ : Eu acho que o fato de a gente conversar assim bem próximo e talvez, não sei assim exatamente, mas acho que a circunstância, a forma como a gente conversa. Eu não sei se eu tenho outro tipo de diálogo assim próximo assim e tão íntimo. Não sei. Talvez. Então me faz ficar um pouco mais contida em relação a isso. $-C R B 2$

T: Mas é curioso porque por um lado você já tá mais exposta, né. Você tá se abrindo mais do que você se abre pra outras pessoas. Então, é uma exposição maior. E aí isso que é tão natural na frente dos outros não acontece aqui. - TRB2

C: É, então. Eu fiquei assim tomando aquela coragem e fiquei decidida a falar mesmo. E quando tinha aqueles momentos que eu via que ia ser uma briga eu parava de falar e falava "eu não vou continuar". E me senti assim bem naquele momento. (..) E como eu não tô acostumada de fazer isso, me deu um pouco essa sensação assim. Foi bom, eu falei o que eu precisava e tudo, mas eu fiquei com aquela incerteza se tudo que eu falei foi certo mesmo. $C R B 2$

T: Fica uma insegurança, né. Tá dando um passo diferente do normal. Eu imagino. - TRB2

C: Mas assim, de forma geral foi acho que mais tranquilizador pra mim. Tava precisando fazer isso. $-C R B 2$

$\mathrm{T}$ : Como ele reagiu? - TPR

C: Bem. Tenho acho que ficado mais calma ultimamente. Mesmo com tudo que vem acontecendo de trabalho e tudo. Acho que tô conseguindo lidar melhor. - CRB2

T: O que você tem sentido que você tá fazendo de diferente? - TRB2

(C. falando sobre a dificuldade de continuar morando com a tia.)

C: Não. Ainda não fiz nenhum tipo de conta, assim, exata. Mas eu preciso fazer. Embora minha mãe sempre fique falando. Quer dizer, minha mãe, o meu namorado, eles sempre falam que é bobagem eu sair de lá e pagar aluguel. Que eu deveria aguentar e comprar um apartamento e tudo. Racionalmente eu penso da mesma forma, mas eu cheguei até a falar pra minha mãe que de repente eu posso até conseguir isso, mas enquanto eu não comprar meu apartamento vou tá com minha cabeça onde? Então... - CRB2

T: Nossa, parece um sacrifício tão grande. Não vale a pena, né? Deve ter uma outra saída. TRB2

C: É. E como é complexo e até um pouco confuso algumas coisas porque envolve várias informações, muitas mesmo, eu acho que é comum. Ela sempre pergunta às vezes as mesmas coisas, mesmo eu já tendo falado. Ela já tem anotado. Ela ainda pergunta. Mas eu prefiro ainda que pergunte do que que faça na incerteza e fazer errado. Então assim. Eu sei dessa dificuldade, mas não me incomoda. Eu acho que é comum. - CRB2

T: É. Me parece uma tolerância pelo começo, né, pelo período de aprendizado dela. Imagino que se isso continuar daqui um ano você não vai ficar tão tranquila. Eu não ficaria no seu lugar. - TRB2 
INF: Terapeuta responde ao CCR de forma grosseiramente ineficaz tanto por reforçar CCR1 como por punir CCR2.

- Quando há um CRB1 por mudança de assunto, e T. emite uma fala que continua o assunto (para o qual foi desviado), só considerar INF se T. não volta no assunto original em até 5 falas corridas. Se T. emite um TRB1, as falas intermediárias são TPR e CPR.

Regra 5: Terapeuta discute (dá exemplo), evoca ou reforça CCR3, O3 ou paralelos entre os dois, buscando por generalização do comportamento do cliente.

T: Quer dizer, deixa eu ver se eu tô realmente entendendo. Em alguns momentos que você acaba ainda cedendo ao que a sua mãe e ele querem, provavelmente outras pessoas também, mas acho que eles são os mais fortes nisso, a dificuldade é pelo que eles vão julgar ao seu respeito. Como se você não tivesse ido na casa da sua mãe ontem e ela cobrando, ela ia te julgar... - Regra 5

C: É. Então não sei. Em algum momento ele sabe que aquilo que me faz bem eu me privo por causa dele. Eu literalmente falei isso pra ele. Então, acho que quando eu tô com ela realmente eu consigo ficar naquele momento. - $\mathrm{O} 2$

T: E com as sobrinhas também? - TO2

C: Também. $-C P R$

T: Eu acho que é isso mesmo, né. Simplesmente curtir o momento. Nada mais elaborado e complexo do que isso. É conseguir reconhecer na hora o que você quer ou gostaria de estar fazendo e fazer. Mesmo que fique lá um incomodozinho de que ele vai ficar chateado. Bom, depois eu lido com isso. Você vai ter que lidar com isso de qualquer jeito, né. - Regra 5 C: É. $-C P R$

C: É. Acho que pra eu conseguir tomar coragem mesmo eu fiquei o tempo todo pensando que seria o melhor pra mim. $-O 3$

T: Bem naquela linha de prestar atenção em você mesma, né. De conseguir estar reconhecendo o dia todo que era isso que você queria. - Regra 5

C: É. $-C P R$

T: Que linda. Que legal. E foi muito pesado o dia de ter passado o dia pensando nisso? Regra 2

T: E que que você acha que te ajudou a conseguir falar? No fundo, o que que falta lá fora no dia a dia pra você conseguir se expressar desse jeito? Seja sobre uma caixinha, sobre um presente ou sobre outras coisas. O que que tem aqui que não tem lá? - Regra 5

C: Não sei exatamente, mas eu sei que eu me sinto mais à vontade aqui. Mesmo com toda a dificuldade que eu tenho eu me sinto mais à vontade de falar com você porque eu me sinto menos julgada. Então, eu sempre tenho um pouco de receio assim do que eu vou falar, de como eu vou falar e do que eu vou receber. É um medo de ser criticada. Eu acho que não sei exatamente, mas eu penso é isso. Não ser positivo assim. $-C R B 3$

T: Como é que você ficou depois da semana passada com aquele exercício de olhar pra dentro de você, como foi depois daquilo? - Regra 5 
C: Ah, não sei ainda, não consegui pensar muito nisso na verdade. É, mas foi mais fácil pra mim assim, lidar com a situação. Então eu acho que fiquei um pouco menos ansiosa, né? Eu sei que no andamento assim, na sequência assim eu me senti melhor, acho que menos apreensiva, menos tensa. Acho que foi um pouco, eu consegui me ver que eu sou muito tensa, que eu sou muito... - CRB3

T: Eu lembro que você comentou mesmo. E essa coisa de ter conseguido se ver mais tensa, né, bastante tensa, você acha que o exercício assim, te ajudou nisso de olhar pra dentro de si? - Regra 5

T: Você acha que pode ter relação a maior tranquilidade no trabalho com a conversa que você teve com ele? - Regra 5

$\mathrm{C}$ : Eu acho que sim. Porque embora eu sempre não misture muito as coisas, mas o clima acho que ficou até um pouco mais suave assim né. -O3

T: Será que nessas horas de ansiedade se fosse possível parar e fazer uma meditação parecida com a que a gente faz aqui te ajudaria? - Regra 5

$\mathrm{C}$ : Acho que se eu conseguisse fazer, sim. $-O 3$ 
APÊNDICE E

TERMO DE CONSENTIMENTO LIVRE E ESCLARECIDO PARA CLIENTES 


\section{TERMO DE CONSENTIMENTO LIVRE E ESCLARECIDO PARA CLIENTE}

Prezado cliente,

Somos professora e estudante de pós-graduação do Laboratório de Terapia Comportamental do Instituto de Psicologia da Universidade de São Paulo (IPUSP) e estamos estudando o processo de mudança do cliente na psicoterapia. Para isto, gostaríamos de pedir sua colaboração voluntária para participação de um projeto de pesquisa de doutorado.

Para que você possa decidir se pode colaborar conosco, procuramos esclarecer resumidamente, a seguir, o que será feito. Se depois de ler, você tiver dúvidas e quiser mais esclarecimentos, estaremos à sua disposição.

\section{Justificativa, objetivos e procedimentos que serão utilizados na pesquisa:}

Nossa pesquisa consiste na condução de um atendimento psicoterápico, com sessões semanais de 50 minutos, realizadas na sala de atendimento do Laboratório de Terapia Comportamental da Clínica-Escola do IPUSP. Tais sessões serão filmadas por uma câmera situada na parte superior da sala. A pesquisa prosseguirá por um período compreendido entre seis meses e um ano e meio e a terapia poderá continuar até o final do semestre corrente ao final da pesquisa, se você o desejar. Se houver a necessidade, após o término deste período, o encaminhamento para outro terapeuta poderá ser feito.

As sessões psicoterápicas serão conduzidas de acordo com os preceitos da Psicoterapia Comportamental e, a partir de determinado momento, será iniciado um trabalho com a própria relação terapêutica (relacionamento existente entre terapeuta e cliente), assim como prevê a Psicoterapia Analítica Funcional (FAP), terapia reconhecida na área nacional e internacional da Análise do Comportamento.

Ao longo da pesquisa, uma de suas tarefas, além de comparecer às sessões será preencher semanalmente uma folha de registro sobre seu próprio comportamento. Tal folha de registro lhe será enviada por correio eletrônico e deverá ser preenchida regularmente. $\mathrm{O}$ preenchimento desse registro é de essencial importância para a pesquisa que estará sendo conduzida ao permitir a identificação de suas melhoras em sua vida diária. Sendo assim, caso tal preenchimento não ocorra por três sessões consecutivas, sem justificativa, a pesquisa será interrompida, dando-se continuidade à terapia até o final do semestre corrente. Ao final do semestre um novo encaminhamento poderá ser realizado, caso seja de seu interesse. Assim, se algo o impedir de preencher os registros, pedimos que tal assunto seja discutido com a terapeuta-pesquisadora em sessão a fim de verificar se será dada ou não continuidade à pesquisa. Solicitamos também que seja indicado por você um Observador. O mesmo deve ser uma pessoa adulta, com quem tenha relacionamento próximo e que seja de sua confiança para que essa pessoa nos ajude a observar seu comportamento em momentos específicos da pesquisa. Os comportamentos a serem registrados serão discutidos com você antes de qualquer informação ser passada à pessoa indicada.

\section{Desconfortos, riscos possíveis e benefícios esperados:}

Nenhum risco específico decorrente do procedimento é previsto na presente pesquisa já que se trata de um processo terapêutico a fim de trazer melhoras em seu dia-a-dia. Portanto, os riscos existentes são semelhantes aos de processos psicoterapêuticos de forma geral. Qualquer procedimento experimental aqui empregado será utilizado apenas de forma preferencial, jamais sendo negado a você qualquer tipo de ajuda que venha a ser identificada, mesmo que esta seja contrária aos objetivos da pesquisa. 
É previsto que você seja beneficiado pelo procedimento proposto já que o objetivo da pesquisa é o de desenvolver melhoras em seu dia-a-dia. Espera-se, portanto que seus relacionamentos interpessoais melhorem, o que demonstraria o sucesso do procedimento testado. Não são esperadas pioras nos relacionamentos interpessoais e caso essas ocorram, o procedimento utilizado será revisto, podendo ser interrompido, dando-se continuidade do processo psicoterápico, se essa for sua vontade.

\section{Métodos alternativos existentes:}

Outras formas de psicoterapia podem levar aos mesmos benefícios (ou semelhantes) aos previstos pelo procedimento da presente pesquisa, de modo que, caso seja de sua escolha, você pode se recusar a participar do presente procedimento, procurando outras formas de psicoterapia.

\section{Liberdade do cliente se recusar a participar ou retirar seu consentimento, em qualquer fase da pesquisa, sem penalização alguma e sem prejuízo ao seu cuidado:}

Mesmo que você concorde em participar, você poderá, a qualquer momento, discutir conosco qualquer questão ou desconforto proveniente da pesquisa e poderá retirar seu consentimento, caso considere necessário, podendo prosseguir com a terapia até o final do semestre corrente na ocasião. A interrupção da pesquisa poderá ocorrer de duas formas, a sua escolha: 1) pela interrupção da coleta de dados, mas mantendo o material já coletado em poder do pesquisador ou 2) pela interrupção da coleta de dados e destruição do material já coletado. Para isso, será importante que você nos comunique sobre sua decisão.

\section{Garantia do sigilo que assegura a privacidade do cliente:}

As informações prestadas por você durante os atendimentos, as gravações de sessões e os registros feitos por você e o observador serão utilizadas para fins didáticos e de pesquisa, incluindo publicações científicas. Essas informações serão tratadas de forma confidencial e os seus dados de identificação não serão divulgados em hipótese alguma. Somente pesquisadores do grupo terão acesso às filmagens, sendo que todos assumirão o compromisso formal de absoluto sigilo sobre qualquer informação pertencente às sessões.

\section{Acompanhamento, assistência, garantia de esclarecimento e seus responsáveis:}

Durante toda a pesquisa a terapeuta-pesquisadora estará atenta e discutirá com sua orientadora as suas reações frente aos procedimentos psicoterápicos propostos. Caso seja observado qualquer prejuízo pra você, o procedimento será imediatamente revisto, podendo ser suspenso. Ambas estarão à disposição durante toda a pesquisa para esclarecimentos adicionais. A terapeuta-pesquisadora poderá ser contatada nas próprias sessões de terapia ou pelo e-mail aleavb@usp.br, enquanto a orientadora poderá ser contatada pelo e-mail sbmeyer@usp.br.

Tal Termo de Consentimento Livre e Esclarecido foi redigido em duas vias igualmente assinadas (para o pesquisador e para o participante), de acordo com o item IV da Resolução 196/96, das Diretrizes e Normas Regulamentadoras de Pesquisas Envolvendo Seres Humanos.

O projeto de pesquisa foi submetido e aprovado pelo Comitê de Ética em Pesquisa com Seres Humanos do Instituto de Psicologia da USP (CEPH-IPUSP), sob o número do processo 07406512.9.0000.5561. O CEPH-IPUSP é credenciado no Conselho Nacional de 
Ética em Pesquisa (CONEP) e fica localizado na Av. Professor Mello Moraes, no 1721 - Bloco G - Sala 27 - Cidade Universitária - São Paulo/SP. Contatos podem ser feitos pelo telefone (11) 3091-4182 ou e-mail: ceph.ip@usp.br. O horário de atendimento ao público é das 8 h30 às 12h e das $13 \mathrm{~h} 30 \mathrm{~h}$ às $16 \mathrm{~h}$.

Cordialmente,

Alessandra Villas Bôas

Psicóloga e aluna de Doutorado do

Departamento de Psicologia Clínica
Profa. Dra. Sonia B. Meyer

Professora Livre Docente do Departamento de Psicologia Clínica

$\mathrm{Eu}$,

R.G.: participarei do projeto de pesquisa

descrito acima e declaro que estou ciente e concordo com as condições apresentadas.

São Paulo, de de 20.

Assinatura do cliente 
APÊNDICE F

TERMO DE COMPROMISSO E RESPONSABILIDADE PARA OBSERVADORES 


\section{TERMO DE COMPROMISSO E RESPONSABILIDADE PARA OBSERVADORES}

Prezado observador,

Somos professora e estudante de doutorado do Laboratório de Terapia Comportamental do Instituto de Psicologia da Universidade de São Paulo e estamos estudando o processo de mudança do cliente na terapia.

O nosso cliente indicou você para nos ajudar a verificar o comportamento dele em sua vida diária. Seu papel será apenas o que registrar alguns dos comportamentos dele, em uma folha de registro, que lhe será enviada por correio eletrônico, em determinados momentos da pesquisa. O ideal é que a ocorrência de tal registro não interfira no relacionamento de vocês. Caso isso aconteça, estaremos dispostos a conversar a respeito e suspender o registro, se for o caso.

As informações prestadas por você no preenchimento da folha de registro serão utilizadas para fins didáticos e de pesquisa, incluindo publicações científicas. Essas informações serão tratadas de forma confidencial e os seus dados de identificação, bem como os do cliente, não serão divulgados. Somente os pesquisadores do grupo terão acesso aos registros, sendo que todos assumirão o compromisso formal de absoluto sigilo sobre qualquer informação registrada.

Porém, pedimos que você respeite também algumas condições para garantir a proteção do cliente e do material utilizado:

1. Apenas eu poderei ter acesso à folha de registro e as minhas respostas;

2. Deverei guardá-las e manuseá-las em local seguro protegido da observação de terceiros;

3. Manterei sigilo absoluto sobre o conteúdo dos registros que farei sobre o cliente;

4. Comprometo-me a não efetuar cópias ou duplicações dos registros.

Mesmo que você concorde em participar, caso sinta qualquer desconforto proveniente da pesquisa, você poderá suspender o registro a qualquer momento. Será importante que você nos comunique sobre a desistência. Você poderá, a qualquer momento, discutir conosco qualquer questão ou dúvida e retirar sua colaboração, caso considere necessário.

Qualquer informação adicional acerca desta pesquisa poderá ser obtida junto aos pesquisadores por meio do e-mail aleavb@usp.br.

Tal Termo de Compromisso foi redigido em duas vias igualmente assinadas (para o pesquisador e para o observador), de acordo com o item IV da Resolução 196/96, das Diretrizes e Normas Regulamentadoras de Pesquisas Envolvendo Seres Humanos.

O projeto de pesquisa foi submetido e aprovado pelo Comitê de Ética em Pesquisa com Seres Humanos do Instituto de Psicologia da USP (CEPH-IPUSP), sob o número do processo 07406512.9.0000.5561. O CEPH-IPUSP é credenciado no Conselho Nacional de Ética em Pesquisa (CONEP) e fica localizado na Av. Professor Mello Moraes, no 1721 - Bloco G - Sala 27 - Cidade Universitária - São Paulo/SP. Contatos podem ser feitos pelo telefone (11) 3091-4182 ou e-mail: ceph.ip@usp.br. O horário de atendimento ao público é das 8 h30 às 12h e das 13h30h às 16h.

Cordialmente, 
Alessandra Villas Bôas

Psicóloga e aluna de Doutorado do

Departamento de Psicologia Clínica
Profa. Dra. Sonia B. Meyer

Professora Livre Docente do Departamento de Psicologia Clínica

$\mathrm{Eu}$,

R.G.: ........................................................................, realizando a atividade de observador para a pesquisa descrita acima me comprometo a respeitar as condições definidas nesse termo.

São Paulo, de de 20 .

Assinatura do observador 
APÊNDICE G

TERMO DE COMPROMISSO E RESPONSABILIDADE PARA TERAPEUTA E AFERIDOR DE CONCORDÂNCIA 


\section{TERMO DE COMPROMISSO E RESPONSABILIDADE}

Prezado aferidor de concordância e pesquisador-terapeuta,

O presente trabalho intitulado "Efeitos de análises de contingências sobre Comportamentos Clinicamente Relevantes e sobre mudanças extra sessão" é uma pesquisa de doutorado da psicóloga Alessandra Villas-Bôas e está sob orientação da Profa. Dra. Sonia Beatriz Meyer, da Universidade de São Paulo. É importante que algumas condições sejam respeitadas para garantir a proteção do cliente e do material utilizado:

1. Apenas eu poderei ter acesso ao conteúdo das sessões e dos registros;

2. Deverei guardá-los e manuseá-los em local seguro protegido da observação de terceiros;

3. Manterei sigilo absoluto sobre toda e qualquer informação mantida nos registros que tive acesso, como por exemplo, identidade do terapeuta e do cliente, dados pessoais de ambos;

4. Comprometo-me a devolver todo o material utilizado sem efetuar cópias ou duplicações e sem qualquer tipo de violação do material original.

$\mathrm{Eu}$, portador do RG:

realizando a atividade de para a pesquisa descrita acima me comprometo a respeitar as condições definidas nesse termo.

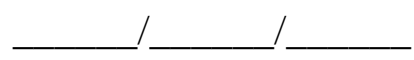

Data
Assinatura
Assinatura do Pesquisador 


\begin{abstract}
APÊNDICE H
TERMO DE COMPROMISSO E RESPONSABILIDADE PARA

TRANSCRITORES
\end{abstract}




\section{TERMO DE COMPROMISSO E RESPONSABILIDADE}

Prezado transcritor,

O presente trabalho intitulado "Efeitos de análises de contingências sobre Comportamentos Clinicamente Relevantes e sobre mudanças extra sessão" é uma pesquisa de doutorado da psicóloga Alessandra Villas-Bôas e está sob orientação da Profa. Dra. Sonia Beatriz Meyer, da Universidade de São Paulo. É importante que algumas condições sejam respeitadas para garantir a proteção do cliente e do material utilizado:

1. Apenas eu poderei ter acesso ao conteúdo das sessões e dos registros;

2. Deverei guardá-los e manuseá-los em local seguro protegido da observação de terceiros;

3. Manterei sigilo absoluto sobre toda e qualquer informação mantida nos registros que tive acesso, como por exemplo, identidade do terapeuta e do cliente, dados pessoais de ambos;

4. Comprometo-me a devolver todo o material utilizado sem efetuar cópias ou duplicações e sem qualquer tipo de violação do material original.

$\mathrm{Eu}$,

portador do RG:

realizando a atividade de transcritor, para a pesquisa descrita acima me comprometo a respeitar as condições definidas nesse termo.

São Paulo, de de

Assinatura do transcritor

Assinatura da pesquisadora 


\section{APÊNDICE I}

FREQUÊNCIA DE EMISSÃO DE CADA SUBCATEGORIA DE REGRA 5, CCR3 E O3, POR FASE EXPERIMENTAL 
Tabela I1: Frequência de emissão de cada subcategoria de Regra 5, CCR3 e 03, por fase experimental, conduzida com a cliente Dora.

\begin{tabular}{|c|c|c|c|c|c|c|c|c|c|c|c|c|c|c|c|c|c|c|c|c|c|c|c|c|c|c|c|c|c|c|c|c|c|c|c|c|c|c|}
\hline \multicolumn{39}{|c|}{ Dora } \\
\hline & & & $\mathbf{A}$ & & & & & & & & & & & & & & & $\overrightarrow{B C 1}$ & & & & & & & & & & & & & & & & & $\mathrm{C2}$ & & & \\
\hline Terapeuta & 1 & 2 & 3 & 4 & 5 & 1 & 2 & 3 & 4 & 5 & 6 & 7 & 8 & 1 & 2 & 3 & 4 & 5 & 6 & 7 & 8 & 9 & 1 & 2 & 3 & 4 & 5 & 6 & 7 & 8 & 1 & 2 & 3 & 4 & 5 & 6 & 7 & 8 \\
\hline Regra 5-P-1 & 0 & 0 & 0 & 0 & 0 & 0 & - & 0 & 0 & 0 & 0 & - & 0 & - & - & - & 0 & 1 & 0 & 0 & 0 & 0 & 0 & 0 & - & - & - & 0 & 0 & 0 & - & 0 & - & 0 & 0 & 0 & - & 0 \\
\hline Regra 5-P-2 & 0 & 0 & 0 & 0 & 0 & 0 & - & 0 & 0 & 0 & 0 & - & 0 & - & - & - & 2 & 1 & 0 & 0 & 2 & 0 & 0 & 0 & - & - & - & 0 & 0 & 0 & - & 0 & - & 1 & 0 & 0 & - & 5 \\
\hline $\begin{array}{l}\text { Regra 5- } \\
\text { CCR3-1 } \\
\end{array}$ & 0 & 0 & 0 & 0 & 0 & 0 & - & 0 & 0 & 0 & 0 & - & 0 & - & - & - & 0 & 0 & 1 & 0 & 0 & 0 & 0 & 0 & - & - & - & 0 & 0 & 0 & - & 0 & - & 1 & 0 & 0 & - & 0 \\
\hline $\begin{array}{l}\text { Regra 5- } \\
\text { CCR3-2 }\end{array}$ & 0 & 0 & 0 & 0 & 0 & 0 & - & 0 & 0 & 0 & 0 & - & 0 & - & - & - & 2 & 3 & 1 & 0 & 1 & 0 & 0 & 0 & - & - & - & 0 & 0 & 0 & - & 2 & - & 0 & 2 & 0 & - & 0 \\
\hline Regra 5-03-1 & 0 & 0 & 4 & 3 & 3 & 0 & - & 4 & 0 & 0 & 0 & - & 0 & - & - & - & 0 & 3 & 15 & 3 & 1 & 3 & 0 & 0 & - & - & - & 0 & 0 & 2 & - & 0 & - & 1 & 0 & 5 & - & 0 \\
\hline Regra 5-03-2 & 5 & 0 & 1 & 1 & 0 & 1 & - & 1 & 1 & 0 & 0 & - & 0 & - & - & - & 1 & 1 & 0 & 0 & 4 & 2 & 0 & 0 & - & - & - & 1 & 0 & 1 & - & 0 & - & 0 & 0 & 1 & - & 1 \\
\hline Regra 5-EP & 0 & 0 & 0 & 0 & 0 & 0 & - & 0 & 0 & 0 & 0 & - & 0 & - & - & - & 0 & 1 & 0 & 0 & 0 & 0 & 0 & 0 & - & - & - & 0 & 0 & 0 & - & 0 & - & 1 & 0 & 0 & - & 0 \\
\hline Regra 5-RP & 0 & 0 & 0 & 0 & 0 & 0 & - & 0 & 0 & 0 & 0 & - & 0 & - & - & - & 0 & 1 & 0 & 0 & 0 & 0 & 0 & 0 & - & - & - & 0 & 0 & 0 & - & 0 & - & 9 & 0 & 0 & - & 0 \\
\hline Regra 5-E3 & 0 & 0 & 0 & 0 & 0 & 0 & - & 1 & 0 & 0 & 0 & - & 0 & - & - & - & 0 & 0 & 0 & 0 & 0 & 1 & 0 & 0 & - & - & - & 0 & 0 & 0 & - & 0 & - & 0 & 0 & 1 & - & 1 \\
\hline Regra 5-R3 & 0 & 1 & 0 & 0 & 0 & 0 & - & 1 & 0 & 0 & 0 & - & 0 & - & - & - & 0 & 0 & 0 & 0 & 0 & 0 & 0 & 0 & - & - & - & 0 & 0 & 0 & - & 0 & - & 5 & 0 & 1 & - & 0 \\
\hline Regra 5-EO3 & 0 & 1 & 0 & 0 & 0 & 0 & - & 0 & 0 & 0 & 0 & - & 0 & - & - & - & 2 & 0 & 2 & 2 & 2 & 1 & 0 & 0 & - & - & - & 0 & 0 & 0 & - & 0 & - & 2 & 0 & 2 & - & 0 \\
\hline Regra 5-RO3 & 0 & 2 & 0 & 1 & 0 & 1 & - & 0 & 0 & 0 & 0 & - & 0 & - & - & - & 2 & 6 & 2 & 1 & 2 & 3 & 0 & 0 & - & - & - & 0 & 0 & 0 & - & 0 & - & 2 & 0 & 0 & - & 1 \\
\hline Cliente Dora & 1 & 2 & 3 & 4 & 5 & 1 & 2 & 3 & 4 & 5 & 6 & 7 & 8 & 1 & 2 & 3 & 4 & 5 & 6 & 7 & 8 & 9 & 1 & 2 & 3 & 4 & 5 & 6 & 7 & 8 & 1 & 2 & 3 & 4 & 5 & 6 & 7 & 8 \\
\hline CCR3-P-1 & 0 & 0 & 0 & 0 & 0 & 0 & - & 0 & 0 & 0 & 0 & - & 0 & - & - & - & 0 & 1 & 0 & 0 & 0 & 0 & 0 & 0 & - & - & - & 0 & 0 & 0 & - & 0 & - & 4 & 0 & 0 & - & 0 \\
\hline CCR3-P-2 & 0 & 0 & 0 & 0 & 0 & 0 & - & 0 & 0 & 0 & 0 & - & 0 & - & - & - & 0 & 0 & 0 & 0 & 0 & 0 & 0 & 0 & - & - & - & 0 & 0 & 0 & - & 0 & - & 4 & 0 & 0 & - & 0 \\
\hline CCR3-1 & 0 & 1 & 0 & 0 & 0 & 1 & - & 0 & 0 & 0 & 0 & - & 0 & - & - & - & 0 & 1 & 0 & 0 & 0 & 1 & 0 & 0 & - & - & - & 0 & 0 & 0 & - & 0 & - & 4 & 0 & 1 & - & 0 \\
\hline CCR3-2 & 0 & 0 & 1 & 0 & 0 & 0 & - & 1 & 0 & 0 & 0 & - & 0 & - & - & - & 0 & 1 & 0 & 0 & 0 & 0 & 0 & 0 & - & - & - & 0 & 0 & 0 & - & 3 & - & 4 & 0 & 0 & - & 1 \\
\hline O3-1 & 4 & 3 & 2 & 0 & 0 & 0 & - & 1 & 0 & 0 & 0 & - & 0 & - & - & - & 3 & 7 & 2 & 2 & 3 & 0 & 0 & 0 & - & - & - & 0 & 0 & 0 & - & 0 & - & 2 & 0 & 0 & - & 3 \\
\hline O3-2 & 1 & 0 & 1 & 2 & 0 & 0 & - & 0 & 0 & 0 & 1 & - & 0 & - & - & - & 0 & 1 & 0 & 0 & 3 & 3 & 0 & 0 & - & - & - & 0 & 0 & 0 & - & 0 & - & 1 & 0 & 2 & - & 0 \\
\hline
\end{tabular}


Tabela I2: Frequência de emissão de cada subcategoria de Regra 5, CCR3 e O3, por fase experimental, conduzida com a cliente Sol.

\begin{tabular}{|c|c|c|c|c|c|c|c|c|c|c|c|c|c|c|c|c|c|c|c|c|c|c|c|c|c|c|c|c|c|c|c|c|c|}
\hline \multicolumn{34}{|c|}{ Sol } \\
\hline & & & $\mathbf{A}$ & & & & & & B1 & & & & & & & $3 \mathrm{C1}$ & & & & & & & B2 & & & & & & & $3 \mathrm{C} 2$ & & & \\
\hline & 1 & 2 & 3 & 4 & 5 & 1 & 2 & 3 & 4 & 5 & 6 & 7 & 1 & 2 & 3 & 4 & 5 & 6 & 7 & 1 & 2 & 3 & 4 & 5 & 6 & 7 & 1 & 2 & 3 & 4 & 5 & 6 & 7 \\
\hline Regra 5-P-1 & 0 & 0 & 0 & 0 & 0 & 0 & 0 & 0 & 0 & 0 & 0 & 0 & 0 & 1 & 0 & 0 & 0 & 0 & 0 & - & 0 & 0 & - & 0 & 0 & 0 & - & - & 0 & 1 & 0 & 0 & 1 \\
\hline Regra 5-P-2 & 0 & 0 & 0 & 0 & 0 & 0 & 0 & 0 & 0 & 0 & 0 & 0 & 0 & 3 & 0 & 0 & 1 & 0 & 2 & - & 0 & 0 & - & 0 & 0 & 0 & - & - & 0 & 1 & 0 & 0 & 1 \\
\hline $\begin{array}{l}\text { Regra 5- } \\
\text { CCR3-1 } \\
\end{array}$ & 0 & 0 & 0 & 0 & 0 & 0 & 0 & 0 & 0 & 0 & 0 & 0 & 0 & 0 & 0 & 0 & 0 & 0 & 0 & - & 0 & 0 & - & 0 & 0 & 0 & - & - & 0 & 0 & 0 & 1 & 0 \\
\hline $\begin{array}{l}\text { Regra 5- } \\
\text { CCR3-2 }\end{array}$ & 0 & 0 & 0 & 0 & 0 & 0 & 0 & 0 & 0 & 0 & 0 & 0 & 0 & 1 & 0 & 2 & 0 & 0 & 0 & - & 0 & 0 & - & 0 & 0 & 0 & - & - & 1 & 0 & 0 & 1 & 0 \\
\hline Regra 5-03-1 & 3 & 2 & 0 & 0 & 4 & 2 & 2 & 0 & 0 & 0 & 0 & 1 & 0 & 0 & 0 & 1 & 1 & 0 & 0 & - & 0 & 0 & - & 0 & 0 & 0 & - & - & 0 & 0 & 1 & 0 & 0 \\
\hline Regra 5-O3-2 & 0 & 0 & 0 & 0 & 0 & 0 & 0 & 0 & 0 & 0 & 0 & 1 & 0 & 0 & 1 & 5 & 1 & 2 & 0 & - & 0 & 0 & - & 0 & 1 & 0 & - & - & 1 & 0 & 3 & 0 & 1 \\
\hline Regra 5-EP & 0 & 0 & 0 & 0 & 0 & 0 & 0 & 0 & 0 & 0 & 0 & 0 & 0 & 0 & 1 & 0 & 0 & 1 & 0 & - & 0 & 0 & - & 0 & 0 & 0 & - & - & 0 & 0 & 0 & 0 & 0 \\
\hline Regra 5-RP & 0 & 0 & 0 & 0 & 0 & 0 & 0 & 0 & 0 & 0 & 0 & 0 & 0 & 0 & 1 & 0 & 1 & 2 & 0 & - & 0 & 0 & - & 0 & 0 & 0 & - & - & 0 & 0 & 0 & 0 & 0 \\
\hline Regra 5-E3 & 0 & 0 & 0 & 0 & 0 & 0 & 0 & 0 & 0 & 0 & 0 & 0 & 2 & 4 & 1 & 2 & 0 & 1 & 1 & - & 0 & 0 & - & 0 & 0 & 0 & - & - & 0 & 1 & 0 & 0 & 0 \\
\hline Regra 5-R3 & 0 & 0 & 0 & 0 & 0 & 0 & 0 & 1 & 0 & 0 & 0 & 0 & 2 & 2 & 2 & 0 & 0 & 0 & 3 & - & 0 & 0 & - & 0 & 0 & 0 & - & - & 0 & 0 & 2 & 2 & 2 \\
\hline Regra 5-EO3 & 1 & 1 & 2 & 0 & 1 & 0 & 0 & 1 & 0 & 0 & 1 & 0 & 2 & 0 & 0 & 1 & 1 & 1 & 2 & - & 0 & 1 & - & 0 & 3 & 0 & - & - & 1 & 2 & 0 & 0 & 1 \\
\hline Regra 5-RO3 & 0 & 1 & 4 & 0 & 0 & 0 & 2 & 1 & 1 & 0 & 1 & 0 & 0 & 2 & 6 & 1 & 4 & 0 & 5 & - & 0 & 1 & - & 0 & 2 & 0 & - & - & 23 & 1 & 4 & 0 & 1 \\
\hline Cliente Sol & 1 & 2 & 3 & 4 & 5 & 1 & 2 & 3 & 4 & 5 & 6 & 7 & 1 & 2 & 3 & 4 & 5 & 6 & 7 & 1 & 2 & 3 & 4 & 5 & 6 & 7 & 1 & 2 & 3 & 4 & 5 & 6 & 7 \\
\hline CCR3-P-1 & 0 & 0 & 0 & 0 & 0 & 0 & 0 & 0 & 0 & 0 & 0 & 0 & 0 & 0 & 0 & 0 & 0 & 1 & 0 & - & 0 & 0 & - & 0 & 0 & 0 & - & - & 0 & 0 & 0 & 0 & 0 \\
\hline CCR3-P-2 & 0 & 0 & 0 & 0 & 0 & 0 & 0 & 0 & 0 & 0 & 0 & 0 & 0 & 0 & 1 & 0 & 1 & 0 & 0 & - & 0 & 0 & - & 0 & 0 & 0 & - & - & 0 & 0 & 0 & 0 & 0 \\
\hline CCR3-1 & 0 & 0 & 0 & 0 & 0 & 0 & 0 & 0 & 0 & 0 & 0 & 0 & 1 & 1 & 0 & 0 & 0 & 0 & 0 & - & 0 & 0 & - & 0 & 0 & 0 & - & - & 0 & 1 & 0 & 0 & 1 \\
\hline CCR3-2 & 0 & 0 & 0 & 0 & 0 & 0 & 1 & 1 & 0 & 0 & 0 & 0 & 1 & 3 & 2 & 1 & 0 & 1 & 3 & - & 0 & 0 & - & 0 & 0 & 0 & - & - & 0 & 0 & 3 & 2 & 3 \\
\hline O3-1 & 3 & 3 & 3 & 0 & 1 & 2 & 4 & 2 & 3 & 0 & 2 & 0 & 0 & 2 & 1 & 1 & 2 & 1 & 2 & - & 0 & 0 & - & 1 & 1 & 0 & - & - & 19 & 2 & 4 & 0 & 1 \\
\hline O3-2 & 0 & 1 & 3 & 0 & 0 & 0 & 0 & 1 & 1 & 0 & 1 & 0 & 0 & 0 & 7 & 4 & 5 & 2 & 6 & - & 1 & 2 & - & 0 & 3 & 0 & - & - & 2 & 1 & 3 & 0 & 2 \\
\hline
\end{tabular}


